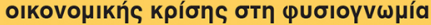
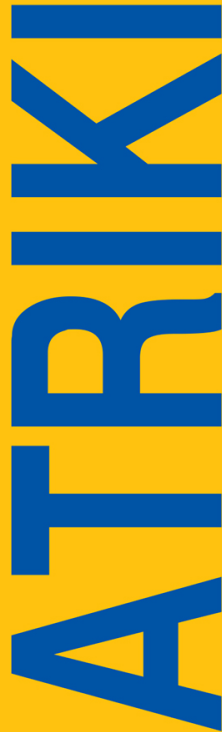

잉 $\stackrel{\oplus}{5}$

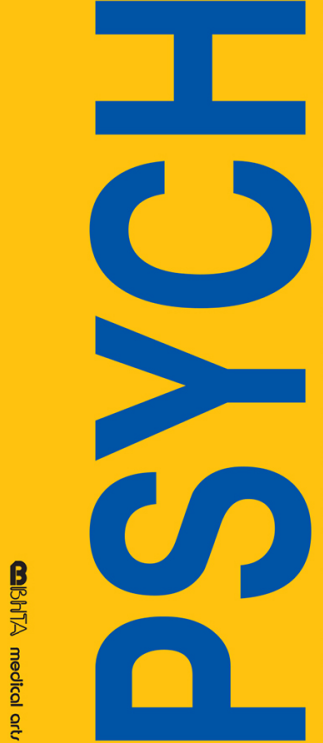

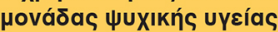

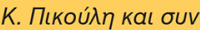

WYXIATPIKH

WYXIATPIKH

WYXIATPIKH

UYXIATPIKH

WYXIATPIKH

WYXIATPIKH

WYXIATPIKH

WYXIATPIKH

WYXIATPIKH

WYXIATPIKH

WYXIATPIKH

WYXIATPIKH

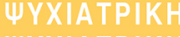

WYXIATPIKH

WY YXIA TPIKH

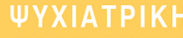

WYXIATPIKH
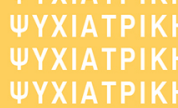

WVXIATPIK H
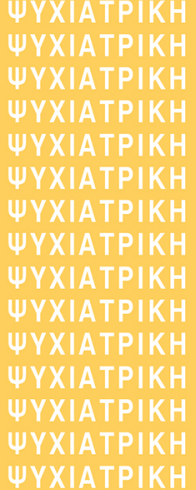

$$
\bullet \bullet
$$

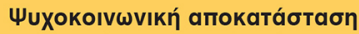

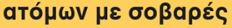

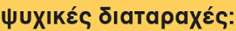

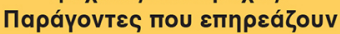

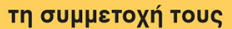

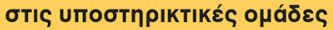

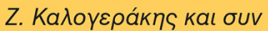

\section{$\bullet \bullet$}

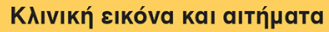

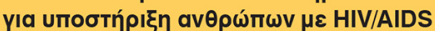

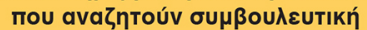

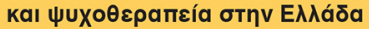

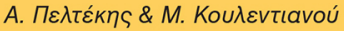

\section{$\bullet \bullet$}

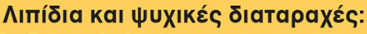

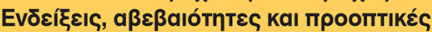

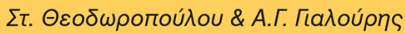

$\bullet \bullet \bullet$

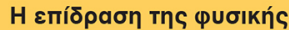

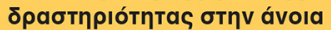

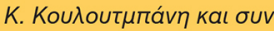

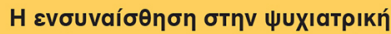

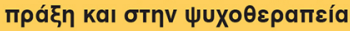
Г. Eoayıáv kaı ouv

$$
\bullet \bullet
$$

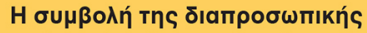

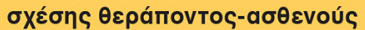

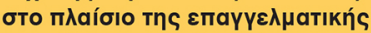

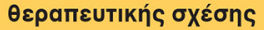

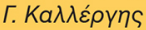
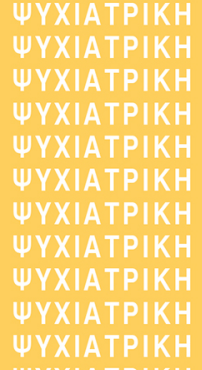

WYXIATPIKH

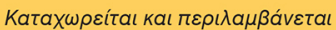
ota MEDLINE/PubMed, Index Copernicus, Scopus,

Google Scholar, EMBASE/Excerpta Medica, GFMER, CIRRIE, SCIRUS

for Scientific Inf., EBSCOhost ${ }^{T M}$,

PsychINFO каı бто latrotek,

(Scopus CiteScore 2018=0.62),

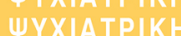

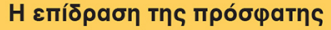

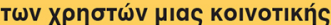

IXXIA TPIKH

WYXIATPIKH

WYXIA TPIKH
WYXIA TPIKH

WYXIA TPIKH
WYXIA TPIKH

IU YXIA TPIKH

WYXIATPIKH

WYXIATPIKH

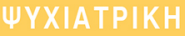

WYXXIA TPIKH

IU YXIA TPIKH
UV YXIA TPIKH
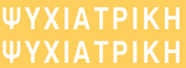

WYXIATPIKH
WYXIATPIKH

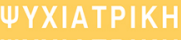

WYXIA TPIKH
WYXIA TPIKH

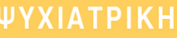

(I) YXIA TPIKH

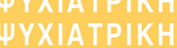

WVXIATPIKH

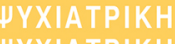

WYXIA TPIKH
WYXIA TPIKH

WY XIA TPIKH

WYXIATPIKH

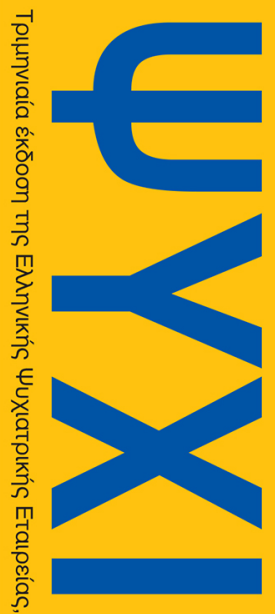

WYXIATPIKH

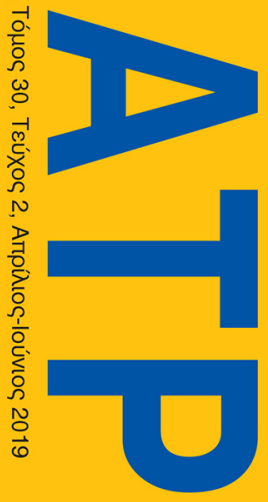

WYXIATPIKH

WYXIATPIKH

WYXIATPIKH

WYXIA TPIKH

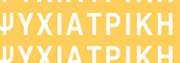

WYXIATPIKH
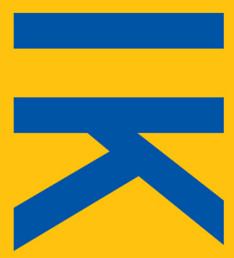

UYXIATPIKH

WYXIA TPIKH
WYXIATPIKH

WYXIATPIKH

WYXIATPIKH

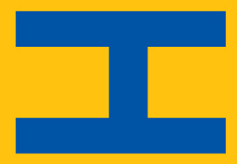

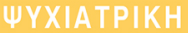

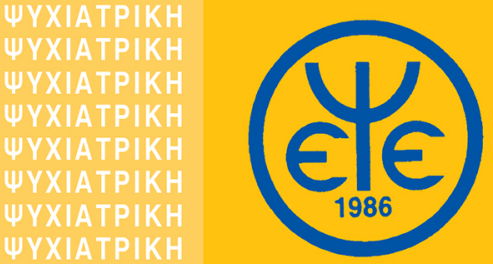




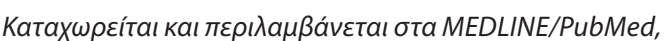
Index Copernicus, Scopus, Google Scholar, EMBASE/Excerpta Medica, GFMER, CIRRIE, SCIRUS for Scientific Inf., EBSCOhost ${ }^{T M}$, PsychINFO Kaı бто latrotek

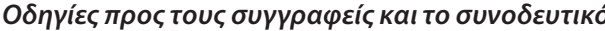

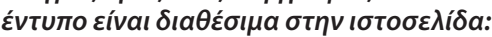
http://www.psychiatriki-journal.gr

\section{YYXIATPIKH}

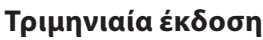

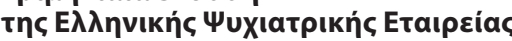

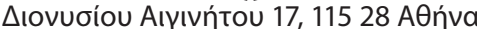

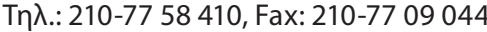

Екठо́тnc:

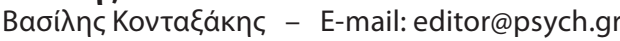

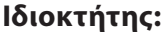

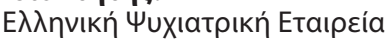

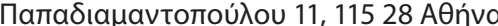

$\operatorname{T\eta } \lambda .:$ 210-72 14184

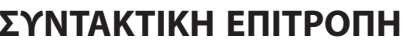

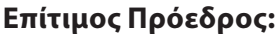

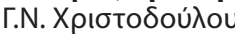

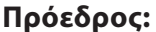

B. Kovta\}áknc

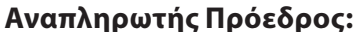

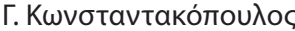

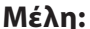

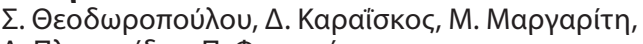

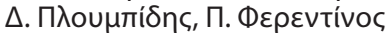

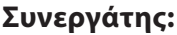

I. Zźpßac

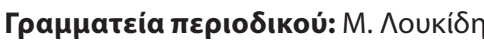

Indexed and included in MEDLINE/PubMed, Index Copernicus, Scopus, Google Scholar, EMBASE/Excerpto Medica, GFMER, CIRRIE, SCIRUS for Scientific Inf., EBSCOhost ${ }^{T M}$, PsychINFO and in latrotek

Instructions to contributors and the submission form are available at the webpage

http://www.psychiatriki-journal.gr

\section{PSYCHIATRIKI}

Quarterly journal published

by the Hellenic Psychiatric Association

17, Dionisiou Eginitou str., 11528 Athens

Tel.: +30-210-77 58 410, Fax: +30-210-77 09044

Publisher:

Vassilis Kontaxakis - E-mail: editor@psych.gr

Owner:

Hellenic Psychiatric Association

11, Papadiamantopoulou str., 11528 Athens

Tel.: +30-210-72 14184

\section{EDITORIAL BOARD}

Emeritus Editor:

G.N. Christodoulou

Editor-in-Chief:

V. Kontaxakis

Associate Editor:

G. Konstantakopoulos

Members:

S. Theodoropoulou, D. Karaiskos, M. Margariti,

D. Ploumpidis, P. Ferentinos

Collaborator:

J. Zervas

Journal's secretariat: M. Loukidi

$\begin{array}{ll}\text { M. Abou-Saleh (UK) } & { }^{\dagger} \text { H. Ghodse (UK) } \\ \text { H. Akiskal (USA) } & \text { P. Gökalp (Turkey) } \\ \text { G. Alexopoulos (USA) } & \text { G. Ikkos (UK) } \\ \text { N. Andreasen (USA) } & \text { R.A. Kallivayalil (India) } \\ \text { S. Bloch (Australia) } & \text { M. Kastrup (Denmark) } \\ \text { M. Botbol (France) } & \text { K. Kirby (Australia) } \\ \text { N. Bouras (UK) } & \text { V. Krasnov (Russia) }\end{array}$

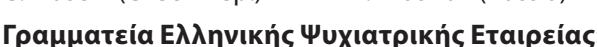

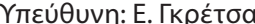

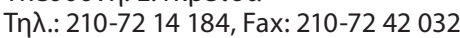

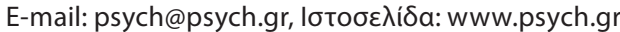
FB: E^^HNIKH $\Psi$ YXIATPIKH ETAIPEIA

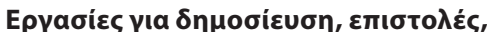

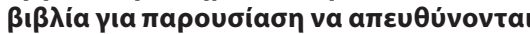

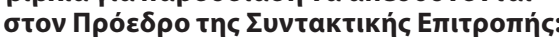
Aıvuoíou Aıүıvńtou 17, 11528 AӨńva

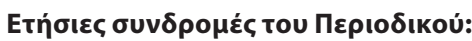

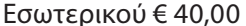

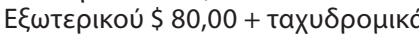

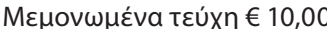

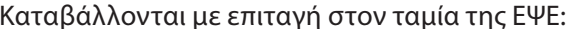

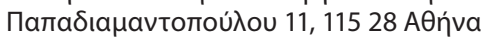

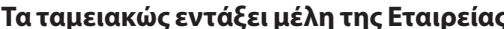

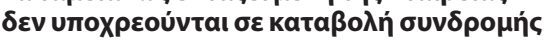

\section{EПIMEAEIA EK $\Delta O \Sigma \mathrm{H} \Sigma$} EN ISO 9001:2000

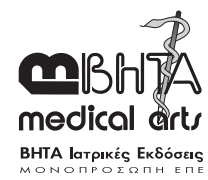

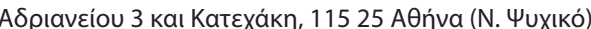
Tn入.: 210-67 14371 - 210-67 14 340, Fax: 210-67 15015 e-mail: betamedarts@otenet.gr e-shop: www.betamedarts.gr

EN ISO 9001:2000

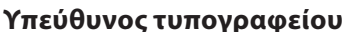

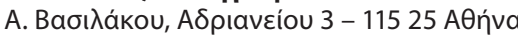

$\operatorname{T\eta } \lambda .210-6714340$
D. Lecic-Tosevski (Serbia) G. Papakostas (USA)

C. Lyketsos (USA)

G. Petrides (USA)
R. Salokangas (Finland)

M. Maj (Italy)

A. Marneros (Germany)

J. Mezzich (USA)

H.J. Möller (Germany) A. Tasman (USA)

O. Steenfeld-Foss (Norway)

R. Montenegro (Argentina) N. Tataru (Romania)

C. Pantelis (Australia) P. Tyrer (UK)

Secretariat of Hellenic Psychiatric Association: Head: H. Gretsa

Tel.: (+30) 210-72 14 184, Fax: (+30) 210-72 42032

E-mail: psych@psych.gr,Web-site:www.psych.gr

Manuscripts, letters, books for review should be addressed to the Editor:

17 Dionisiou Eginitou str., GR-115 28 Athens, Greece

Annual subscriptions of the Journal:

$€ 40.00$ or $\$ 80.00$ + postage - each separate issue $€ 10.00$ are payable by check to the treasurer

of the Hellenic Psychiatric Association:

11, Papadiamantopoulou str., GR-115 28 Athens

For the members of the Association in good standing subscription is free

\section{EDITING}

EN ISO 9001:2000

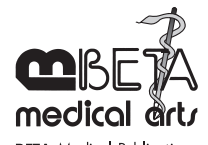

3. Adrianiou str, GR-115 25 Athens-Greece

Tel.: (+30) 210-67 14371 - (+30) 210-67 14340

Fax: (+30) 210-67 15015

e-mail: betamedarts@otenet.gr, e-shop:www.betamedarts.gr EN ISO 9001:2000

Printing supervision

A. Vassilakou, 3 Adrianiou str. - GR-115 25 Athens

Tel. (+30)-210-67 14340 
EAMHNIKH YYXIATPIKH ETAIPEIA

\section{DIOIKHTIKO IYMBOYNIO}

\begin{tabular}{|c|c|}
\hline 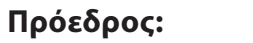 & 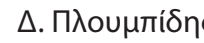 \\
\hline 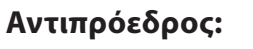 & 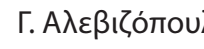 \\
\hline 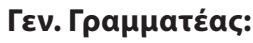 & 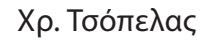 \\
\hline Tapiac: & ^. Марка́кп \\
\hline 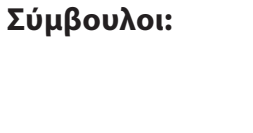 & 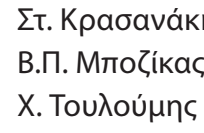 \\
\hline ПEIOAPXIKO $\Sigma Y$ & IMBOYAIO \\
\hline 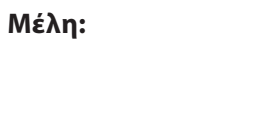 & 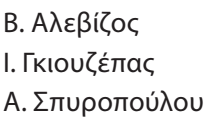 \\
\hline 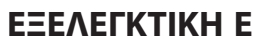 & ПІТРОПН \\
\hline 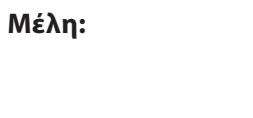 & 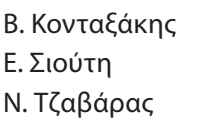 \\
\hline
\end{tabular}

ERITIMOI ПPOE $\triangle$ POI

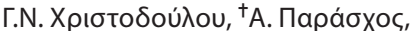

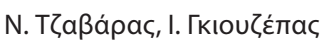

\section{EПITIMA MEAH}

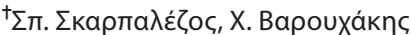

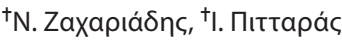

\begin{tabular}{|c|c|}
\hline ПЕРІФЕРЕІ & MATA \\
\hline $\mathrm{A} \Theta \mathrm{HN} \Omega \mathrm{N}$ & \\
\hline 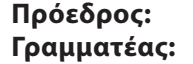 & 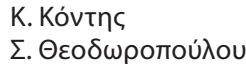 \\
\hline Tapiac: & 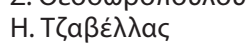 \\
\hline MAKE $\Delta O N I A \Sigma$ & \\
\hline 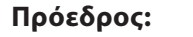 & 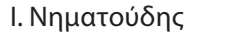 \\
\hline 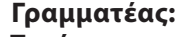 & 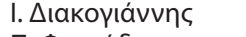 \\
\hline Tapiac: & 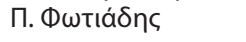 \\
\hline KENTPIKH $\Sigma$ E $\wedge$ & \\
\hline Про́єठрос: & 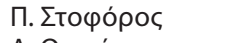 \\
\hline 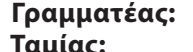 & 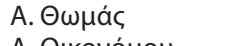 \\
\hline Tapíac: & А. Оıкоvó \\
\hline
\end{tabular}

BOPEIO $\triangle$ ҮTIKH $\Sigma$ E $\wedge$ A $\triangle \mathrm{O} \Sigma \& \triangle \mathrm{YTIKH} \Sigma \Sigma T$ TEPEA $\Sigma$

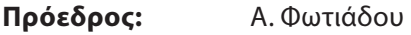

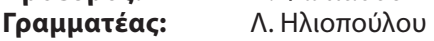

Tauiac: $\quad$ П. Пвтрі́кns

ПE^OПONNHEOY

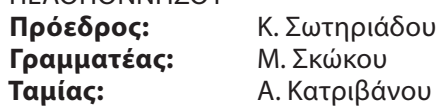

MEГA^H $\Sigma$ BPETTANIA $\Sigma$

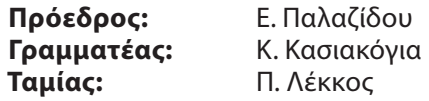

Taulac:

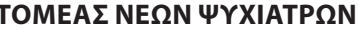

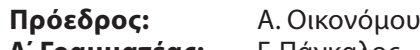

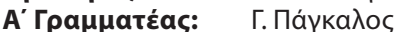

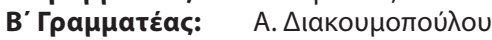

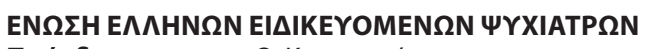

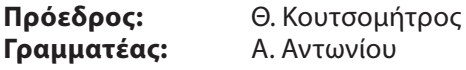

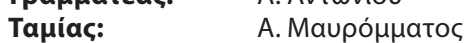

\section{HELLENIC PSYCHIATRIC ASSOCIATION}

\section{EXECUTIVE COUNCIL}

\author{
Chairman: D. Ploumpidis \\ Vice-Chairman: G. Alevizopoulos \\ Secretary General: Ch. Tsopelas \\ Treasurer: L. Markaki \\ Consultants: St. Krasanakis \\ V.P. Bozikas \\ Ch. Touloumis

\section{DISCIPLINARY COUNCIL

$\begin{array}{ll}\text { Members: } & \text { V. Alevizos } \\ & \text { J.Giouzepas } \\ & \text { A. Spyropoulou }\end{array}$

FINANCIAL CONTROL COMMITTEE

$\begin{array}{ll}\text { Members: } & \text { V. Kontaxakis } \\ & \text { Ir. Siouti } \\ & \text { N. Tzavaras }\end{array}$

HONORARY PRESIDENTS

G.N. Christodoulou, ${ }^{\dagger}$ A. Paraschos, N. Tzavaras, J. Giouzepas

\section{HONORARY MEMBERS}

†S. Scarpalezos, Ch. Varouchakis,

${ }^{\dagger} \mathrm{N}$. Zachariadis, ${ }^{\dagger}$ I. Pittaras

\section{DIVISIONS}

ATHENS

$\begin{array}{ll}\text { Chairman: } & \text { C. Kontis } \\ \text { Secretary: } & \text { S. Theodoropoulou } \\ \text { Treasurer: } & \text { E. Tzavellas } \\ \text { MACEDONIA } & \\ \text { Chairman: } & \text { J. Nimatoudis } \\ \text { Secretary: } & \text { J. Diakoyiannis } \\ \text { Treasurer: } & \text { P. Fotiadis } \\ \text { CENTRAL GREECE } & \\ \text { Chairman: } & \text { P. Stoforos } \\ \text { Secretary: } & \text { A. Thomas } \\ \text { Treasurer: } & \text { A. Oikonomou }\end{array}$

NORTHWESTERN GREECE

$\begin{array}{ll}\begin{array}{l}\text { Chairman: } \\ \text { Secretary: }\end{array} & \text { A. Fotiadou } \\ \text { Treasurer: } & \text { P. Petrioulou } \\ \text { PELOPONNESE } & \\ \text { Chairman: } & \text { K. Sotiriadou } \\ \text { Secretary: } & \text { M. Skokou } \\ \text { Treasurer: } & \text { A. Katrivanou } \\ \text { GREAT BRITAIN } & \\ \text { Chairman: } & \text { H. Palazidou } \\ \text { Secretary: } & \text { K. Kasiakogia } \\ \text { Treasurer: } & \text { P. Lekkos }\end{array}$

SECTOR OF YOUNG PSYCHIATRISTS

$\begin{array}{ll}\text { Chairman: } & \text { A. Economou } \\ \text { Secretary A': } & \text { G. Pagkalos } \\ \text { Secretary B': } & \text { A. Diakoumopoulou }\end{array}$

UNION OF GREEK PSYCHIATRIC TRAINEES Chairman: Th. Koutsomitros

Secretary: Ath. Antoniou

Treasurer: $\quad$ A. Mavromatos 


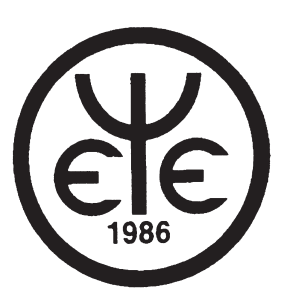

KAA $\Delta O$

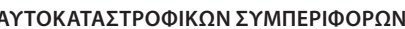

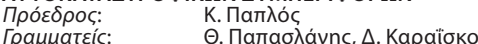

BIAISN $\Sigma Y M \Pi E P I \Phi O P \Omega N$

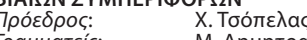

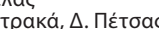

BIONOГIKH $\Sigma$ YYXIATPIKH $\Sigma$

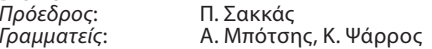

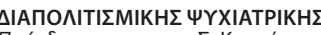

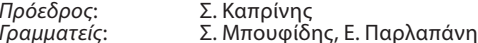

$\triangle$ IATAPAXE $\Pi P O \Sigma \Lambda H \Psi H \Sigma$ TPOФH

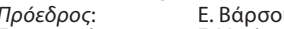

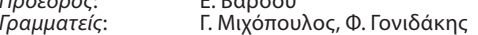

EГKAIPH ПАРЕMBA $\Sigma \mathrm{H} \Sigma T H N \Psi \curlyvee X \Omega \Sigma H$

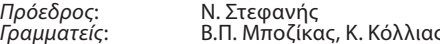

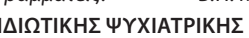

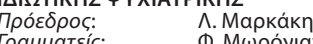

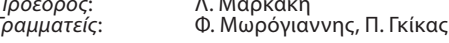

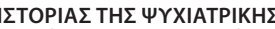

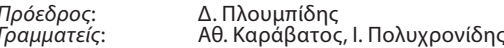

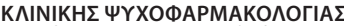

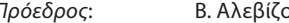

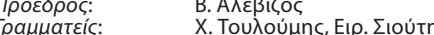

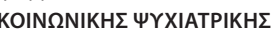

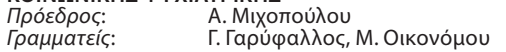

MEAETH $\Sigma$ TH $\Sigma$ EПAГTEAMATIKH $\Sigma$ ПPOA $\Sigma \Pi I \Sigma H \Sigma$ TOY $\Psi$ YXIATPO

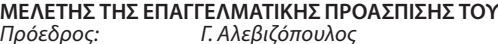

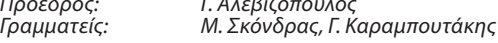

OVIIOEEAPTH $\Sigma E \Omega N$

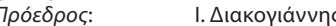

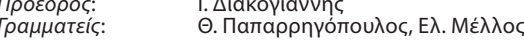

ПAI $\triangle$ OUYXIATPIKH $\Sigma$

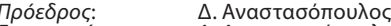

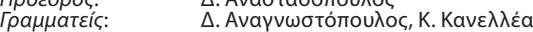

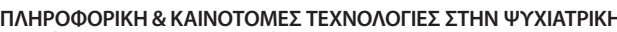

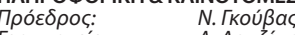

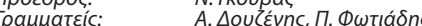

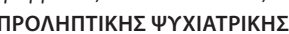

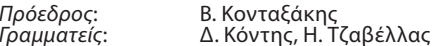

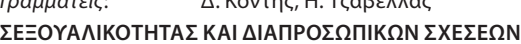

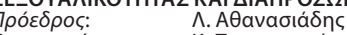

К. Патабтанátnc, H. Мoupíkn

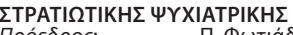

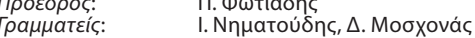

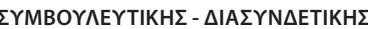

UYXIATPIKH \& \& YYXOEOMATIKH

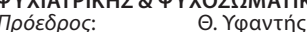

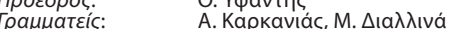

TEXNH $\Sigma \& \Psi Y X I A T P I K H \Sigma$

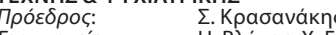

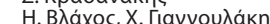

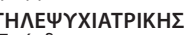

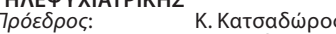

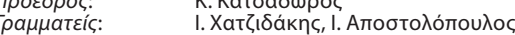

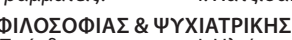

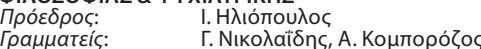

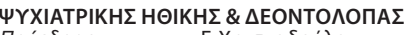

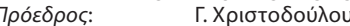

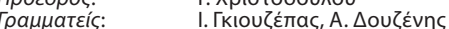

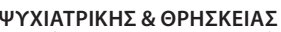

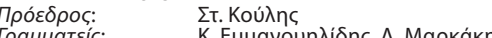

K. E $\mu \mu a v$

$\Psi$ YXIKH $Y$ YГEIA $\Sigma$ ГYNAIK $\Omega N \&$
$\Psi$ YXIATPIKH $\Sigma$ TH $\Sigma$ ANAПAPAГSГH $\Sigma$

ПҮXIATPIKH $\mathrm{TH} \Sigma$ ANAПAPA

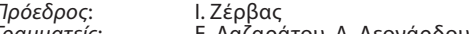

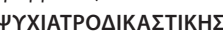

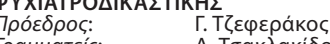

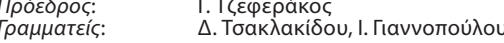

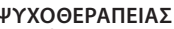

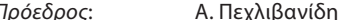

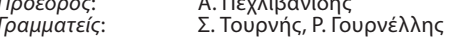

$\psi Y$ XOГHPIATPIKH $\Sigma$

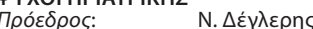

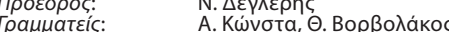

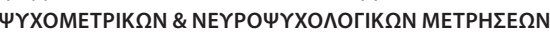

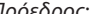

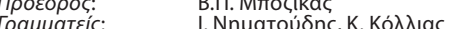

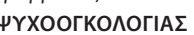

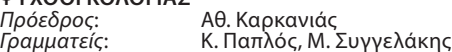

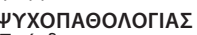

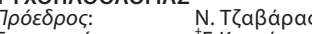

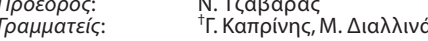

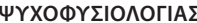

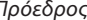

I. Niámiac

Граннатві́

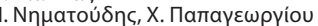

SECTIONS

\begin{tabular}{|c|c|}
\hline $\begin{array}{l}\text { SELF-DESTRUCTIV } \\
\text { Chairman: } \\
\text { Secretaries: }\end{array}$ & $\begin{array}{l}\text { 3EHAVIORS } \\
\text { K. Paplos } \\
\text { Th. Papaslanis, D. Karaiskos }\end{array}$ \\
\hline $\begin{array}{l}\text { VIOLENT BEHAVIORS } \\
\text { Chairman: } \\
\text { Secretaries: }\end{array}$ & $\begin{array}{l}\text { S Ch. Tsopelas } \\
\text { M. Dimitraka, D. Petsas }\end{array}$ \\
\hline $\begin{array}{l}\text { BIOLOGICAL PSYCHI } \\
\text { Chairman: } \\
\text { Secretaries: }\end{array}$ & $\begin{array}{l}\text { IATRY } \\
\text { P. Sakkas } \\
\text { A. Botsis, C. Psarros }\end{array}$ \\
\hline $\begin{array}{l}\text { CROSS-CULTURAL PS } \\
\text { Chairman: } \\
\text { Secretaries: }\end{array}$ & $\begin{array}{l}\text { SYCHIATRY } \\
\text { S. Kaprinis } \\
\text { S. Boufidis, H. Parlapani }\end{array}$ \\
\hline $\begin{array}{l}\text { EATING DISORDERS } \\
\text { Chairman: } \\
\text { Secretaries: }\end{array}$ & $\begin{array}{l}\text { E. Varsou } \\
\text { J. Michopoulos, F. Gonidakis }\end{array}$ \\
\hline $\begin{array}{l}\text { EARLY INTERVENTIO } \\
\text { Chairman: } \\
\text { Secretaries: }\end{array}$ & $\begin{array}{l}\text { N IN PSYCHOSIS } \\
\text { N. Stefanis } \\
\text { V.P. Bozikas, K. Kollias }\end{array}$ \\
\hline $\begin{array}{l}\text { PRIVATE PRACTICE P } \\
\text { Chairman: } \\
\text { Secretaries: }\end{array}$ & $\begin{array}{l}\text { SYCHIATRY } \\
\text { L. Markaki } \\
\text { F. Morogiannis, P. Gkikas }\end{array}$ \\
\hline $\begin{array}{l}\text { HISTORY OF PSYCHIA } \\
\text { Chairman: } \\
\text { Secretaries: }\end{array}$ & $\begin{array}{l}\text { ATRY } \\
\text { D. Ploumpidis } \\
\text { Ath. Karavatos, J. Polyhronidis }\end{array}$ \\
\hline $\begin{array}{l}\text { PSYCHOPHARMACOI } \\
\text { Chairman: } \\
\text { Secretaries: }\end{array}$ & $\begin{array}{l}\text { LOGY } \\
\text { V. Alevizos } \\
\text { C. Touloumis, I. Siouti }\end{array}$ \\
\hline $\begin{array}{l}\text { SOCIAL PSYCHIATRY } \\
\text { Chairman: } \\
\text { Secretaries: }\end{array}$ & $\begin{array}{l}\text { A. Michopoulou } \\
\text { G. Garyfallos, M. Economou }\end{array}$ \\
\hline $\begin{array}{l}\text { ADVOCACY OF PSYCI } \\
\text { Chairman: } \\
\text { Secretaries: }\end{array}$ & $\begin{array}{l}\text { HIATRIC PRACTICE } \\
\text { G. Alevizopoulos } \\
\text { M. Skondras, G. Karampoutakis }\end{array}$ \\
\hline $\begin{array}{l}\text { SUBSTANCE ABUSE } \\
\text { Chairman: } \\
\text { Secretaries: }\end{array}$ & $\begin{array}{l}\text { J. Diakoyiannis } \\
\text { Th. Paparrigopoulos, El. Mellos }\end{array}$ \\
\hline $\begin{array}{l}\text { HILD PSYCHIATRY } \\
\text { hairman: }\end{array}$ & D. Anastasopoulos \\
\hline
\end{tabular}

Secretaries: D. Anagnostopoulos, K. Kanellea

INFORMATICS \& INNOVATIVE TECHNOLOGIES IN PSYCHIATRY

Chairman: $\quad$ N. Gouvas

PREVENTIVE PSYCHIATRY

$\begin{array}{ll}\text { Chairman: } & \text { V. Kontaxakis } \\ \text { Secretaries: } & \text { D. Kontis, E. Tzavellas }\end{array}$

SEXUALITY AND INTERPERSONAL RELATIONSHIPS

Chairman: L. Athanasiadis

Secretaries: K. Papastamatis, H. Mourikis

MILITARY PSYCHIATRY

J. Nimatoudis, D. Moschonas

CONSULTATION-LIAISON PSYCHIATRY

A. Karkanias, M. Diallina

ART \& PSYCHIATRY

S. Krasanakis

Secretaries: Klachos, C. Giannoulak

TELEPSYCHIATRY

Chairman: $\quad$ K. Katsadoros

J.Chatzidakis, J. Apostolopoulos

PHILOSOPHY \& PSYCHIATRY

Chairman: J.lliopoulos

Secretaries: $\quad$ G. Nikolaidis, A. Komborozos

PSYCHIATRY \& ETHICS

G. Christodoulou

Secretaries: J.Giouzepas, A. Douzenis

PSYCHIATRY \& RELIGION

Chairman: $\quad$ S. Koulis

WOMEN'S MENTAL HEALTH \&

REPRODUCTIVE PSYCHIATRY

Chairman: J.Zervas

FORENSIC PSYCHIATRY

Chairman: G. Tzeferakos

$\begin{array}{ll}\text { Secretaries: } & \text { D. Tsaklakidou, J. Giannopoulou }\end{array}$

PSYCHOTHERAPY

Secretaries: $\quad$ S. Tournis, R. Gournellis

A. Konsta, Th Vorvolakos

PSYCHOMETRIC \& NEUROPSYCHOLOGICAL MEASUREMENTS

Chairman:

Secretaries: V.P.Bozikas $\quad$ Nimatoudis, K. Kollias

PSYCHO-ONCOLOGY

Chairman: $\quad$ A. Karkanias

Kecretaries: $\quad$ K. Paplos, M. Syngelakis

PSYCHOPATHOLOGY

$\begin{array}{ll}\text { Chairman: } & \text { N. Tzavaras } \\ \text { Secretaries: } & { }^{+} \text {G. Kaprinis, M. Diallina }\end{array}$

PSYCHOPHYSIOLOGY

J.Liappas

Secretaries: J.Nimatoudis, C. Papageorgiou 


\section{(⿶凵)}

\section{YYXIATPIKH}

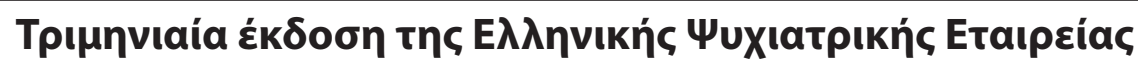

\section{ПEPIEXOMENA}

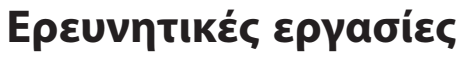

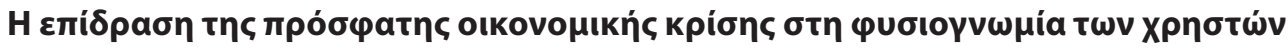

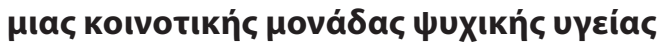

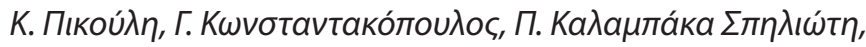

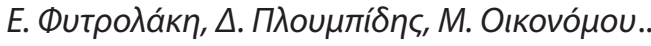

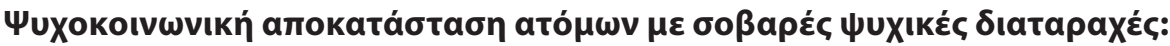

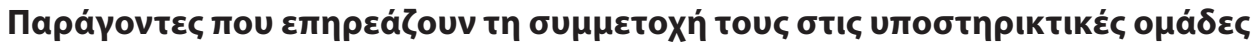

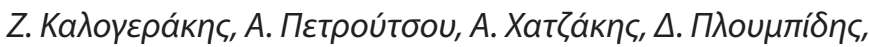

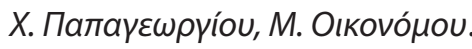

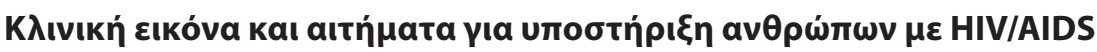

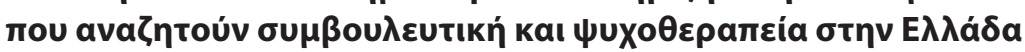

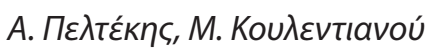

\section{Avaбкómnon}

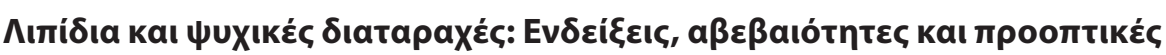

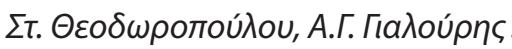

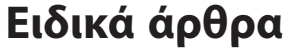

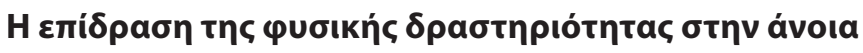

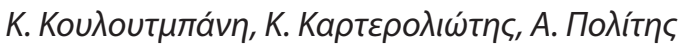

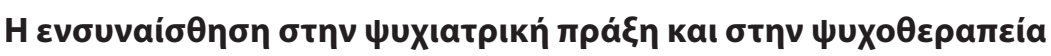

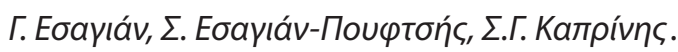

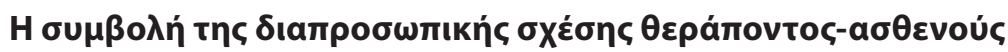

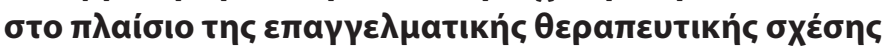




\section{(46)}

\section{PSYCHIATRIKI}

\section{Quarterly journal published by the Hellenic Psychiatric Association}

\section{CONTENTS}

\section{Research articles}

The impact of the recent financial crisis on the users' profile of a community mental health unit

K. Pikouli, G. Konstantakopoulos, P. Kalampaka Spilioti,

E. Fytrolaki, D. Ploumpidis, M. Economou.

Psychosocial rehabilitation and severe mental illness:

Factors that influence patients' attendance in a support group

Z. Kalogerakis, A. Petroutsou, A. Chatzakis, D. Ploumpidis,

Ch. Papageorgiou, M. Economou

Clinical presentation and requests for support of people with HIV/AIDS seeking counselling and psychotherapy in Greece

A. Peltekis, M. Koulentianou .....

\section{Review}

Lipids and mental disorders: Evidence, uncertainties and perspectives

St. Theodoropoulou, A.G. Gialouris

\section{Special articles}

The effect of physical activity on dementia

K. Kouloutbani, K. Karteroliotis, A. Politis

Empathy in psychiatry and psychotherapy

G. Esagian, S. Esagian-Pouftsis, S.G. Kaprinis.

The contribution of the relationship between therapist-patient and the context of the professional relationship

G. Kallergis 


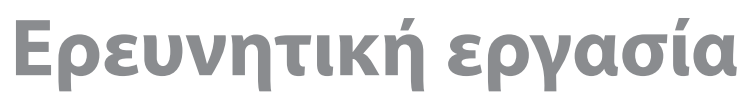 Research article
}

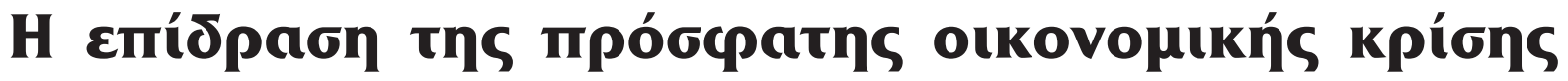

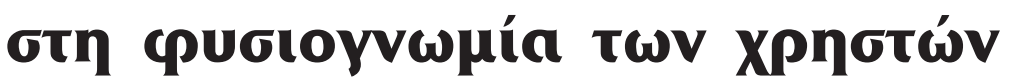

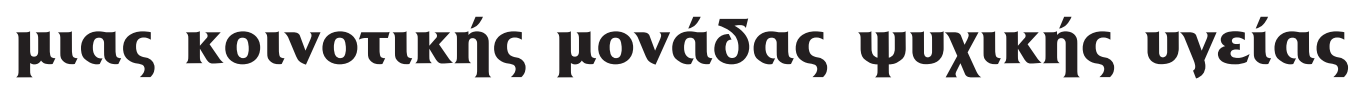

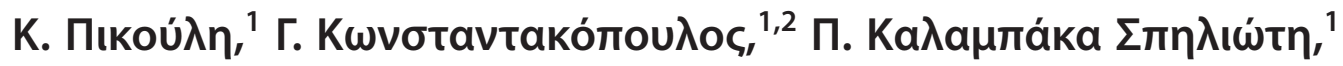

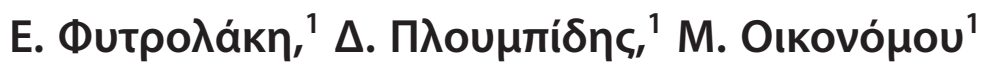

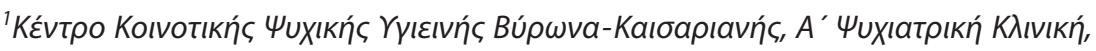

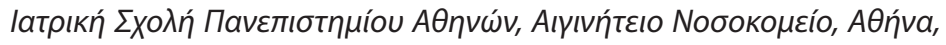 \\ ${ }^{2}$ Department of Psychosis Studies, Institute of Psychiatry, Psychology and Neuroscience, King's College London, UK
}

Uuxıатрıкń 2019, 30:97-107

\footnotetext{
$>$

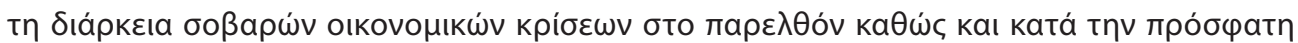

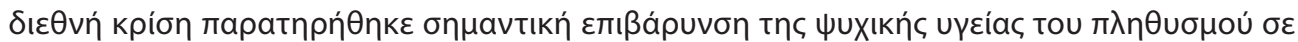

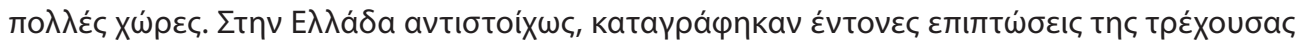

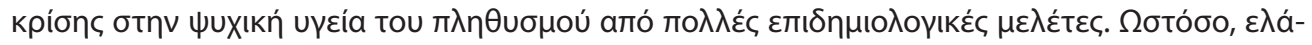

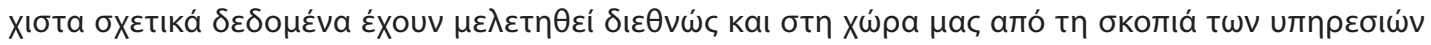

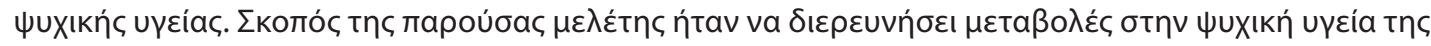

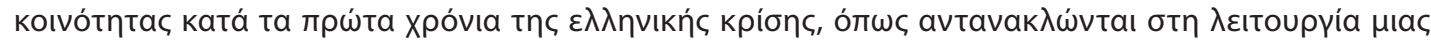

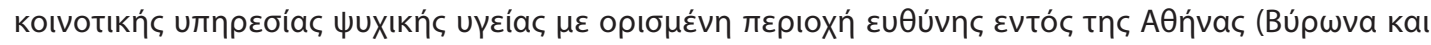

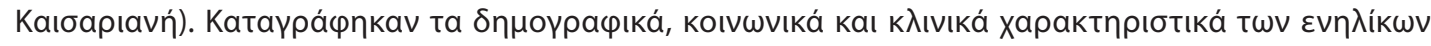

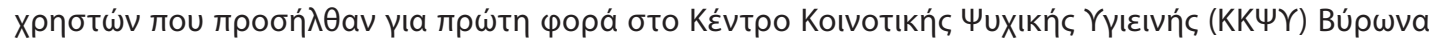

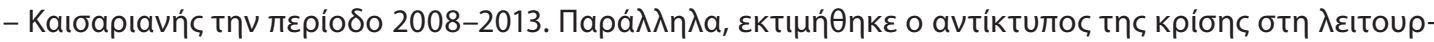

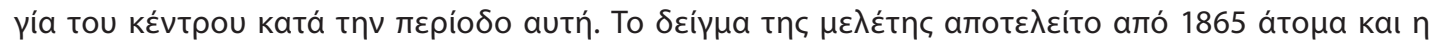

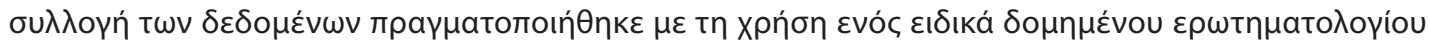

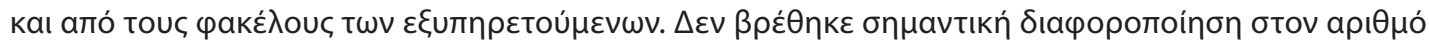

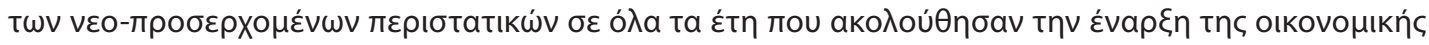

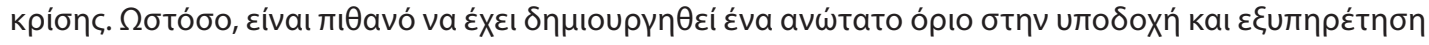




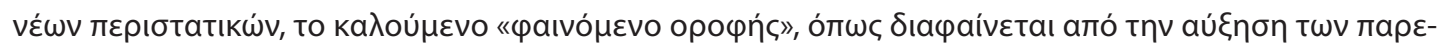

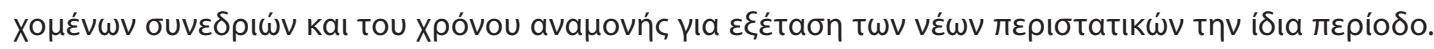

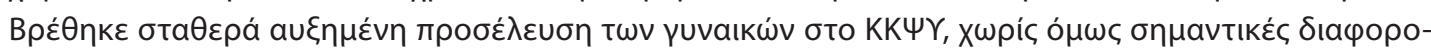

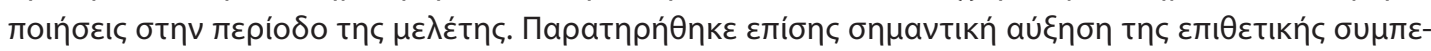

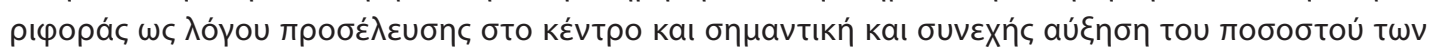

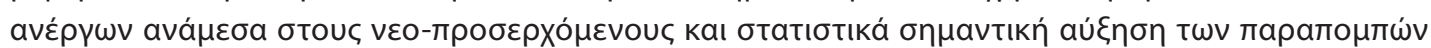

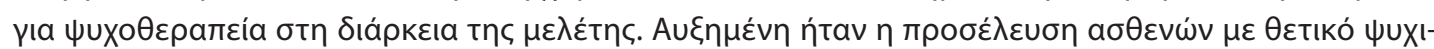

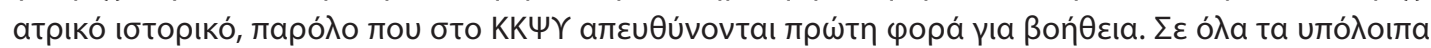

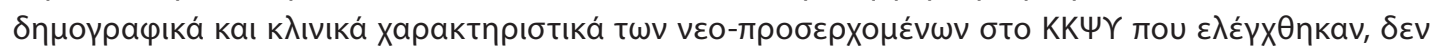

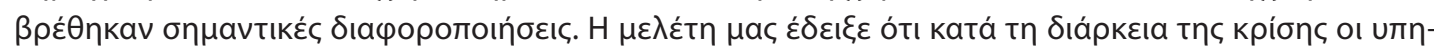

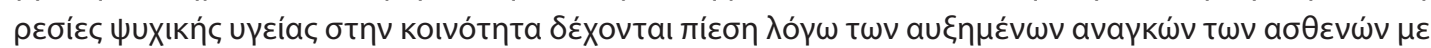

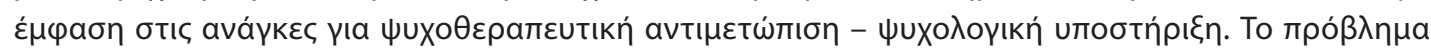

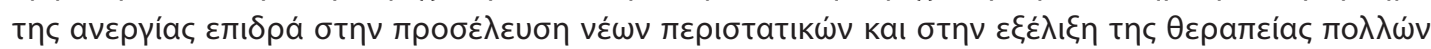

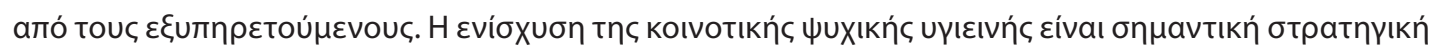

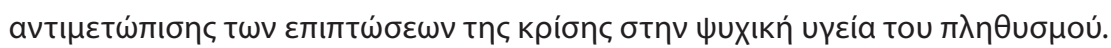

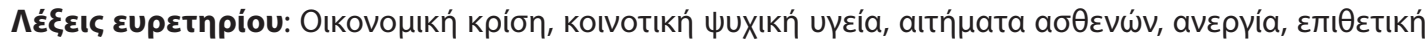
бинтвріфора́.

\section{Eıøaywyń}

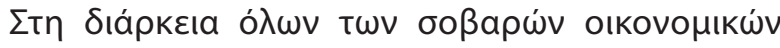

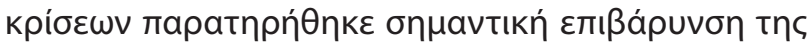

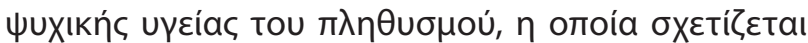

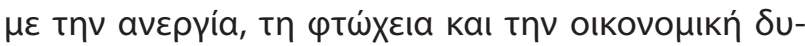

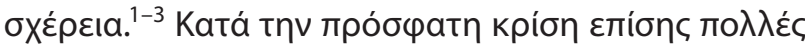

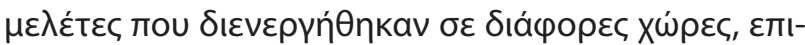

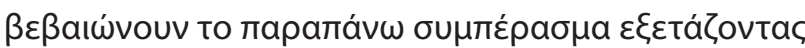

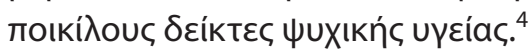

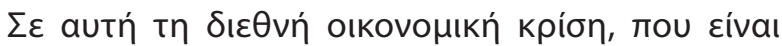

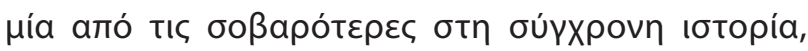

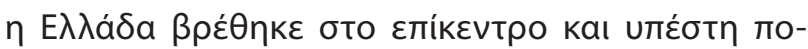

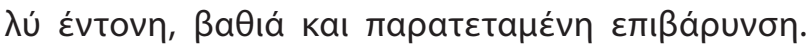

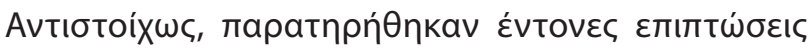

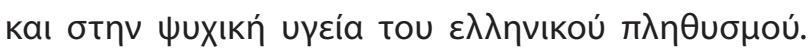

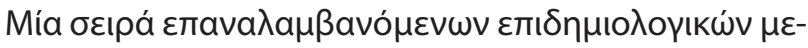

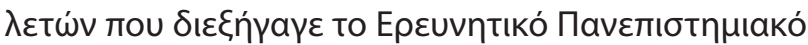

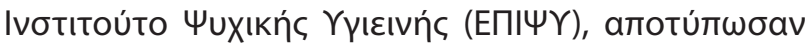

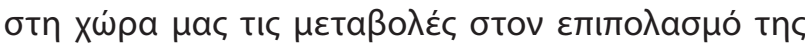

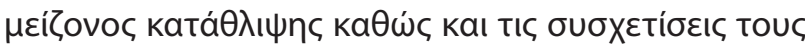

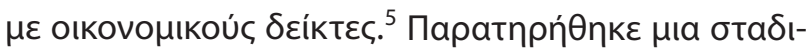

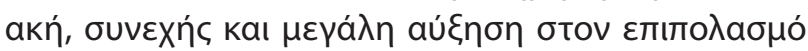

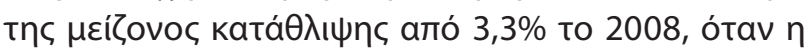

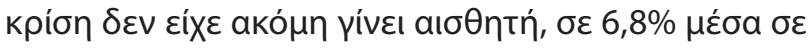

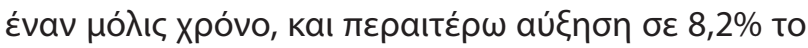

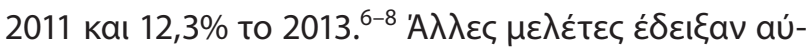

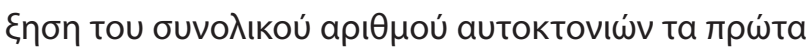

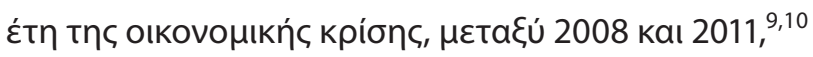

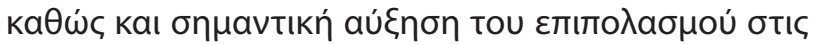

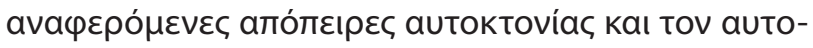

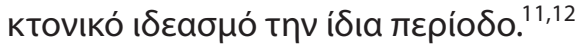

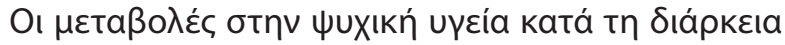

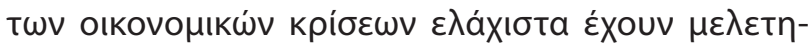

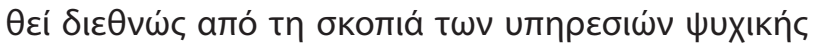

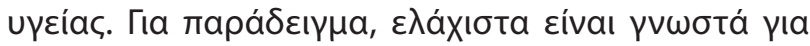

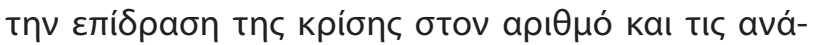

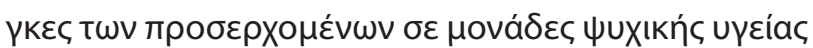

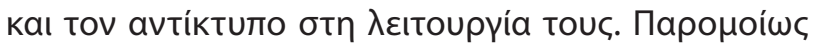

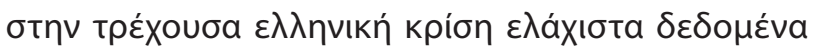

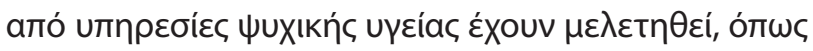

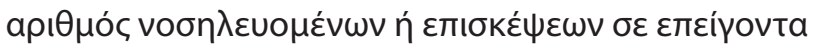

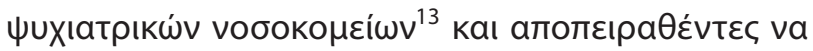

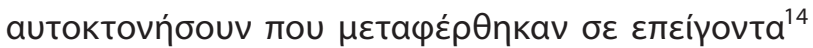

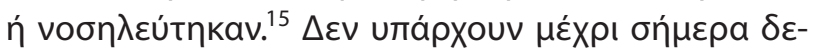

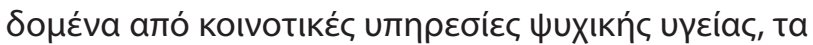

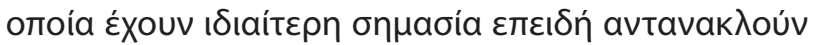

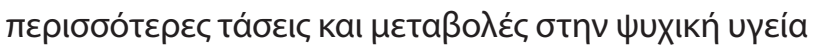

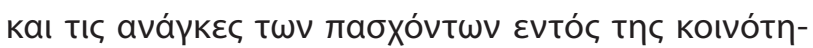

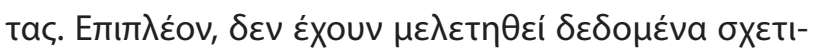

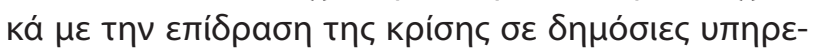

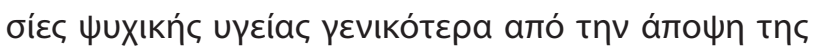

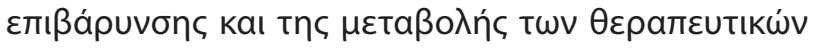

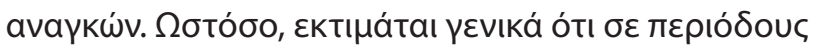

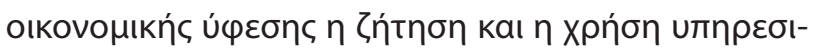

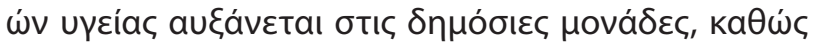




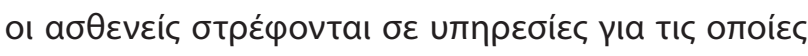

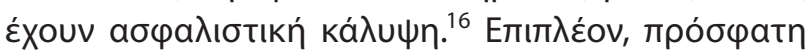

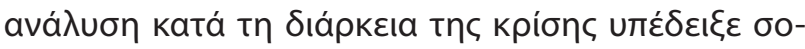

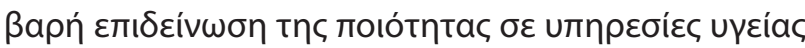

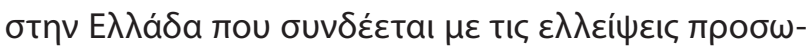

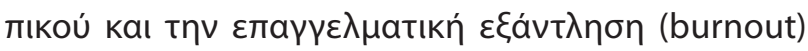

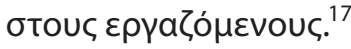

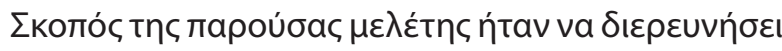

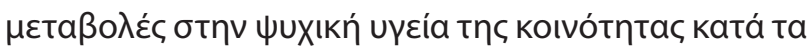

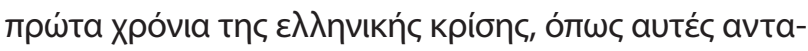

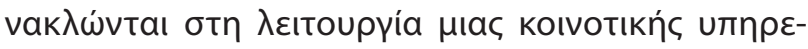

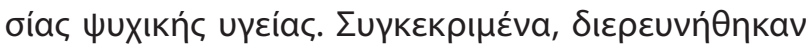

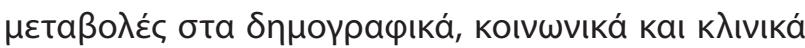

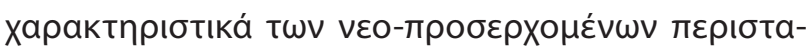

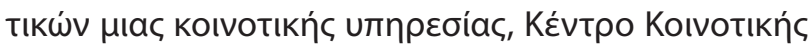

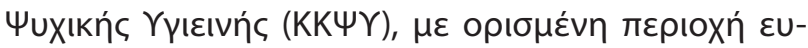

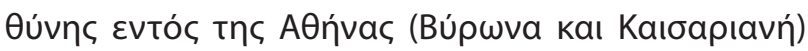

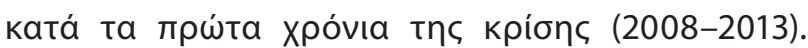

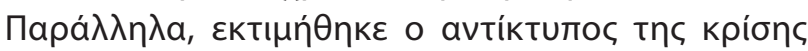

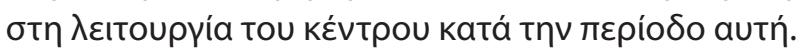

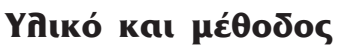

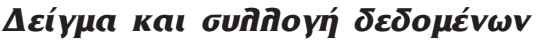

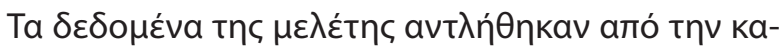

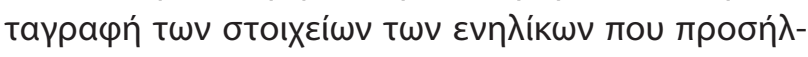

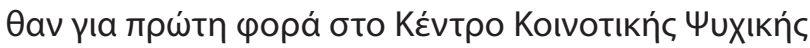

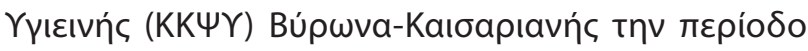

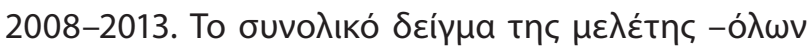

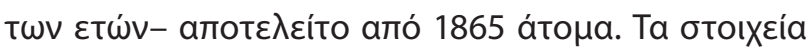

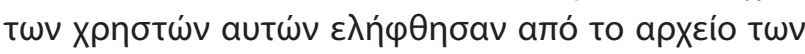

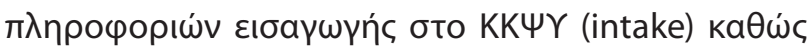

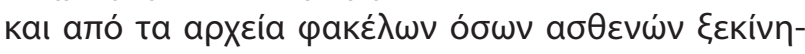

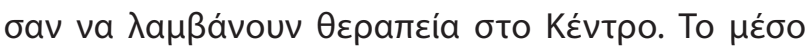

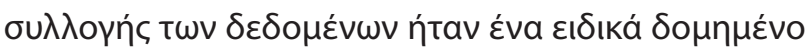

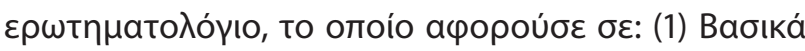

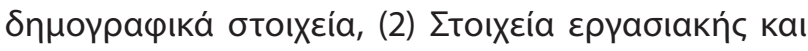

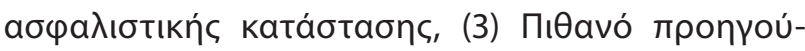

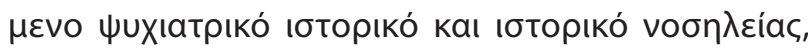

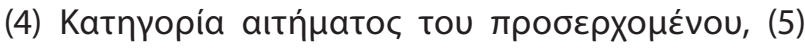

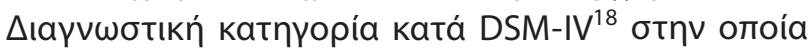

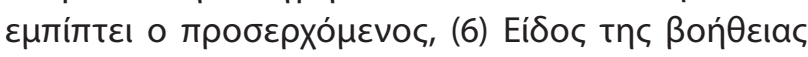

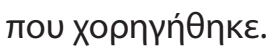

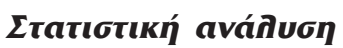

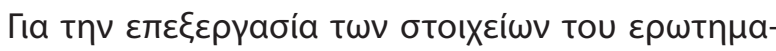

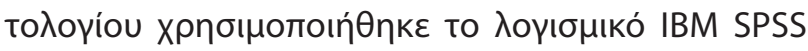
Statistics version 22 for Windows. To ह́toc $2008 \theta \varepsilon \omega$ -

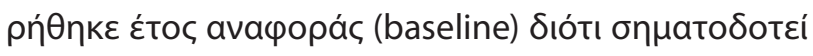

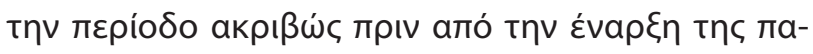

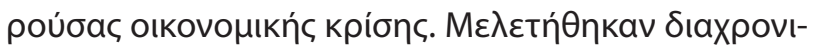

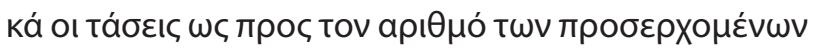

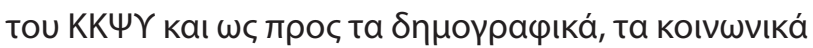

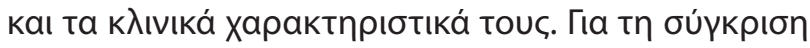

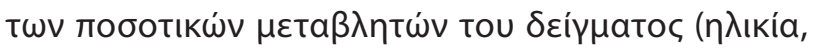

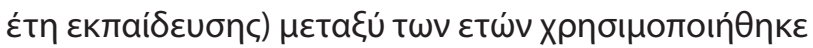

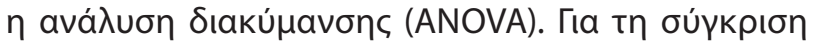

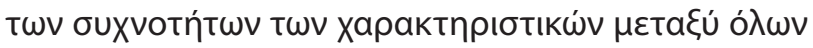

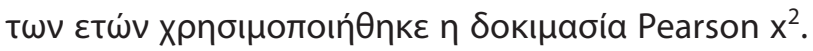

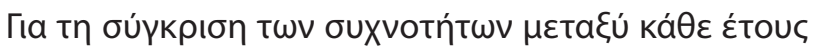

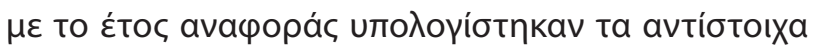

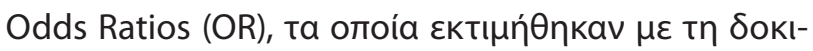

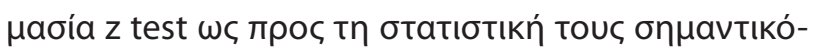

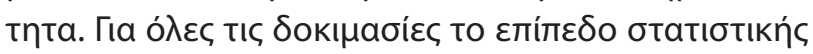

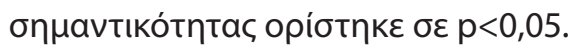

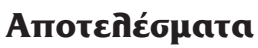

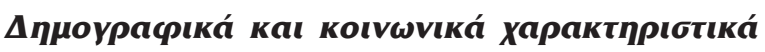

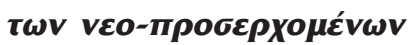

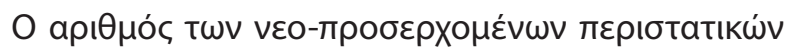

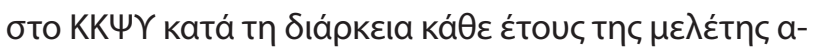

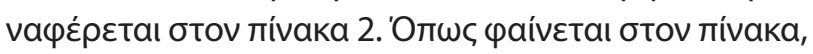

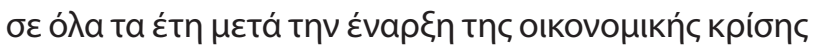

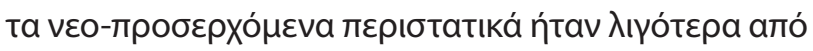

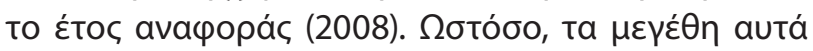

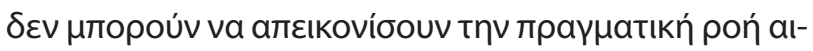

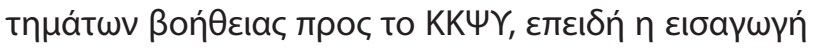

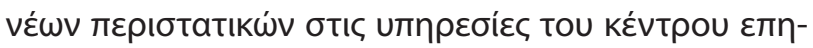

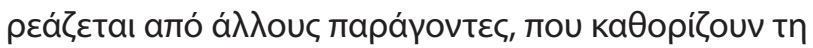

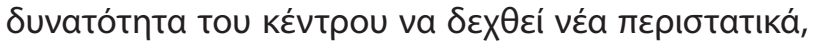

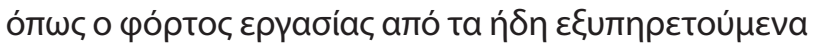

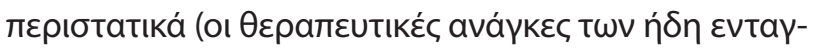

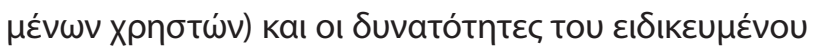

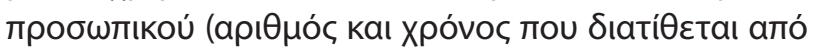

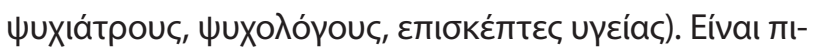

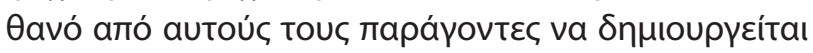

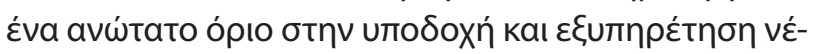

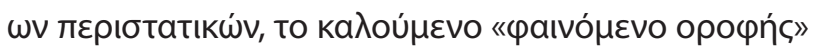
(ceiling effect).

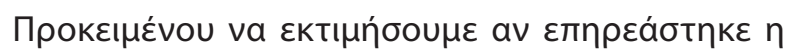

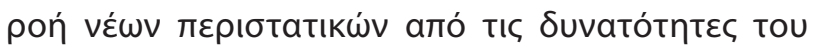

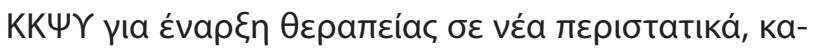

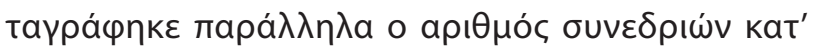

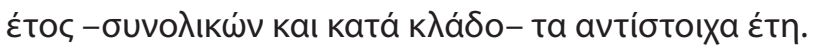

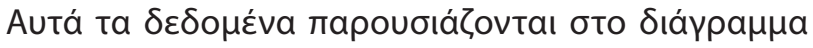




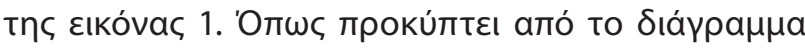

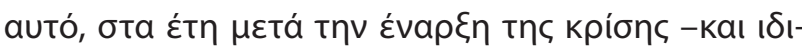

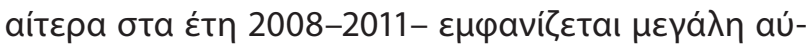

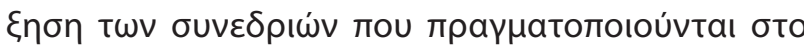

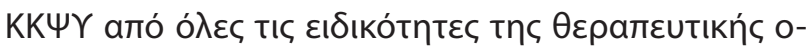

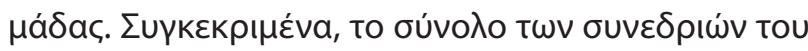

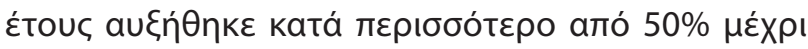

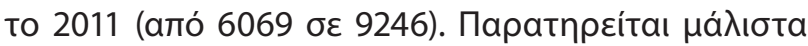

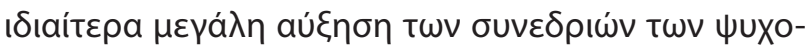

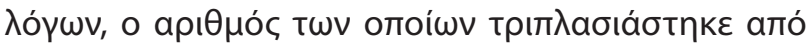

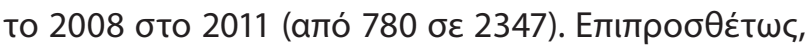

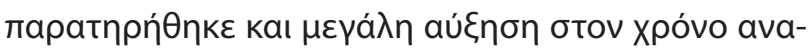

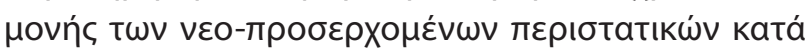

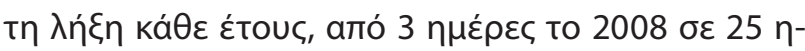

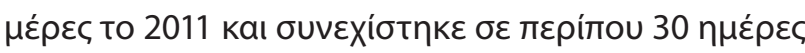

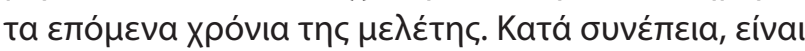

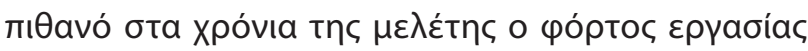

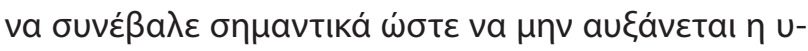

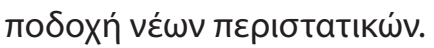

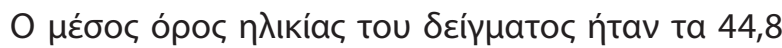

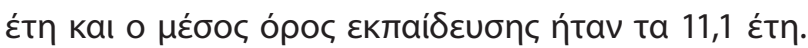

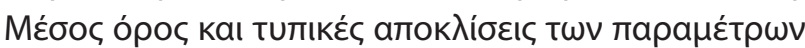

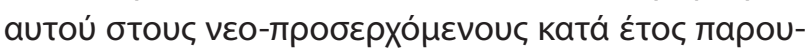

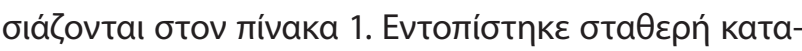

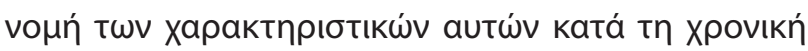

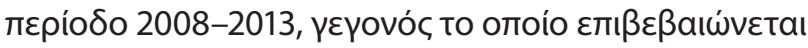

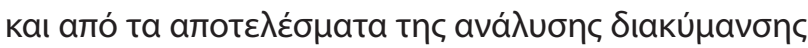

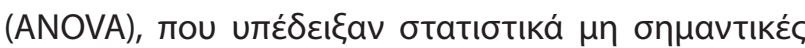

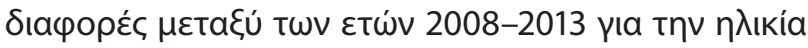

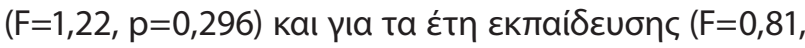

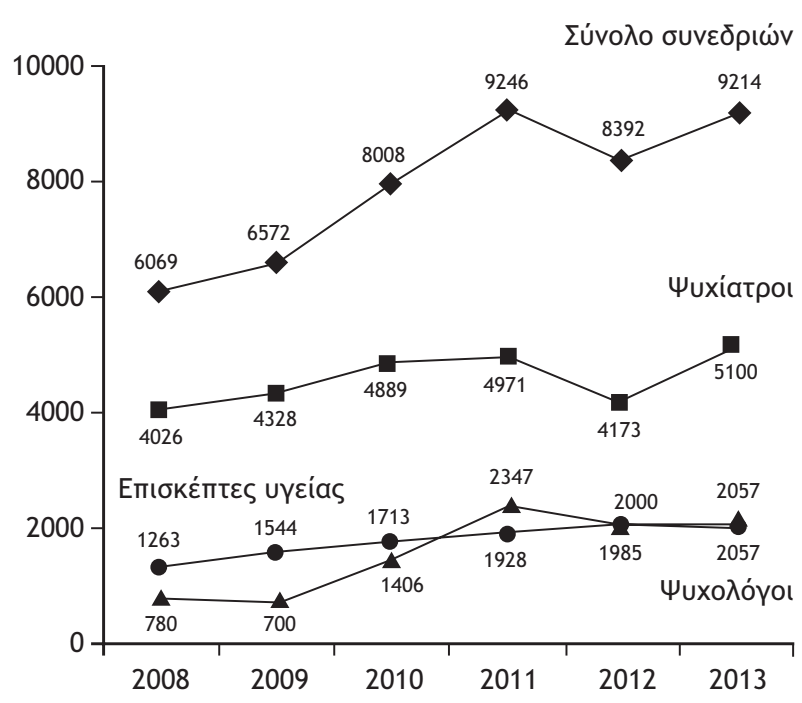

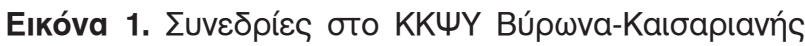

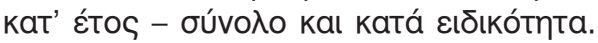

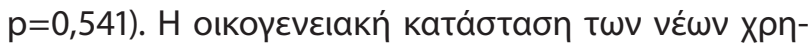

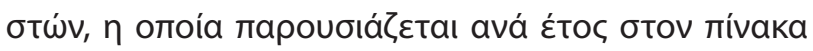

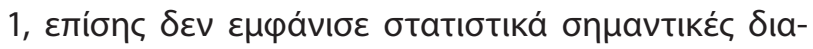

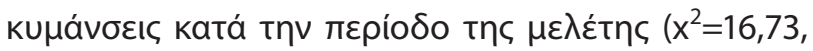
$\mathrm{p}=0,976)$.

'Oбov a

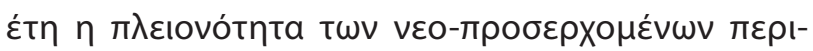

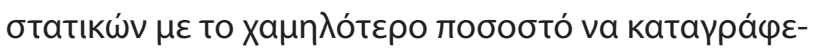

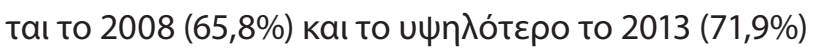

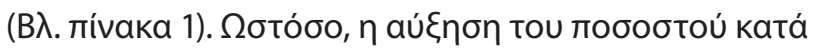

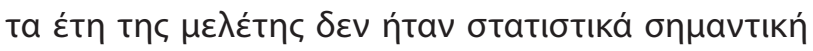
$\left(x^{2}=4,23, p=0,517\right)$.

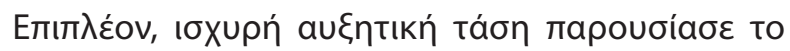

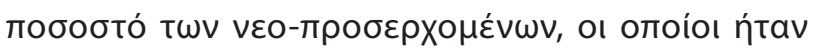

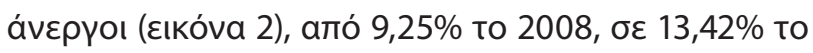

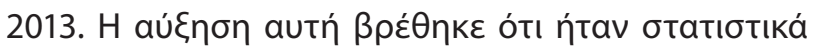

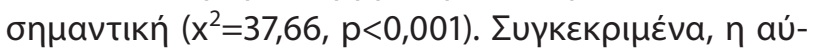

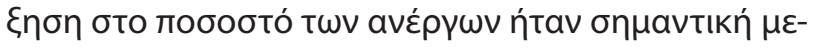

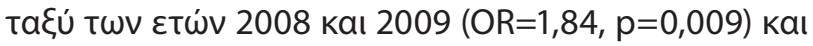

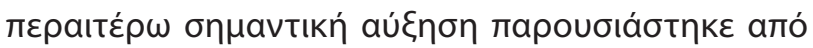
To 2009 бто 2010 (OR=1,52, $\mathrm{p}=0,036)$.

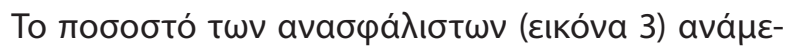

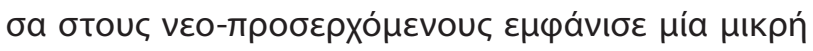

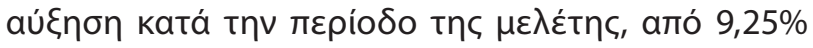

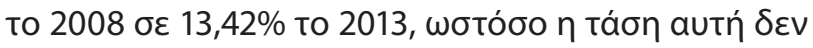

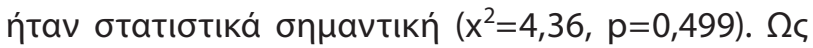

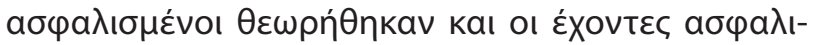

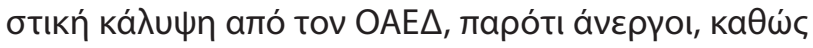

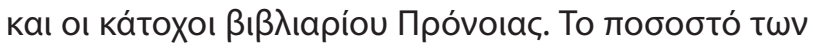

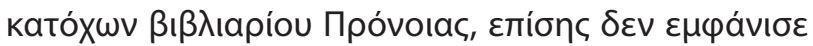

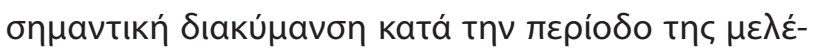

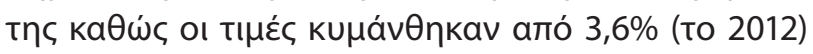

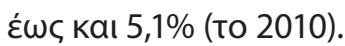

\section{Kลıเкка́ характпрıбтка́

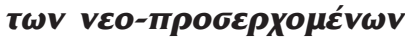

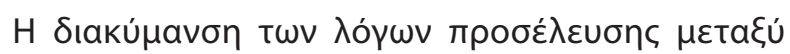

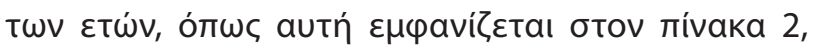

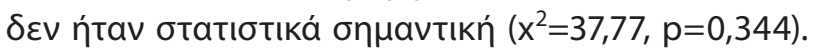

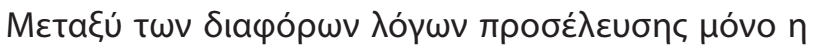

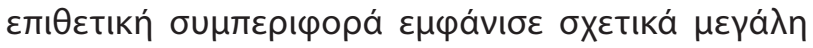

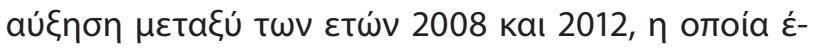

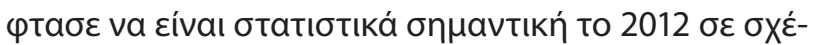

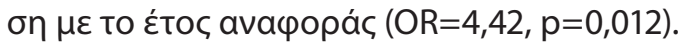

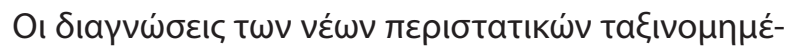

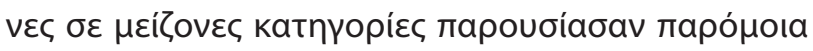

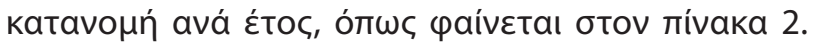




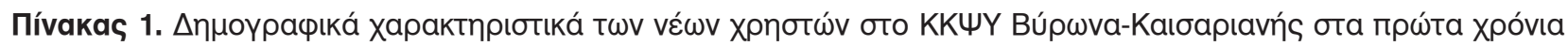
tᄁৎ kpíons.

\begin{tabular}{|c|c|c|c|c|c|c|c|c|c|c|c|c|}
\hline & \multicolumn{2}{|c|}{2008} & \multicolumn{2}{|c|}{2009} & \multicolumn{2}{|c|}{2010} & \multicolumn{2}{|c|}{2011} & \multicolumn{2}{|c|}{2012} & \multicolumn{2}{|c|}{2013} \\
\hline & MO & TA & MO & TA & MO & TA & MO & TA & MO & TA & MO & TA \\
\hline H入ıкі́a & 44,5 & 17,4 & 45,2 & 16,6 & 46,4 & 16,4 & 43,9 & 16,7 & 43,9 & 16,5 & 45,0 & 16,8 \\
\hline \multirow[t]{2}{*}{ 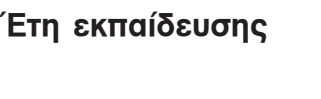 } & 11,1 & 3,8 & 10,9 & 3,9 & 10,9 & 3,8 & 11,3 & 3,6 & 11,4 & 3,7 & 11,4 & 7,2 \\
\hline & $\mathbf{n}$ & (\%) & $\mathbf{n}$ & (\%) & $\mathbf{n}$ & (\%) & $\mathbf{n}$ & (\%) & $\mathbf{n}$ & (\%) & $\mathbf{n}$ & (\%) \\
\hline 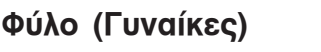 & 237 & 65,9 & 236 & 66,3 & 210 & 70,4 & 219 & 68,2 & 159 & 70,0 & 217 & 71,9 \\
\hline \multicolumn{13}{|c|}{ 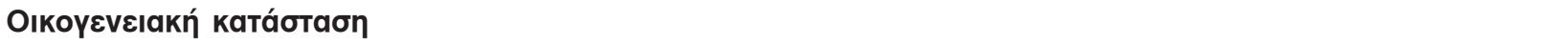 } \\
\hline 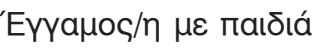 & 127 & 36,2 & 128 & 36,3 & 111 & 37.9 & 113 & 35,5 & 79 & 35,0 & 116 & 39,1 \\
\hline 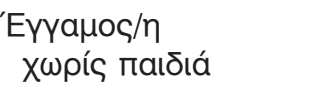 & 18 & 5,1 & 20 & 5,7 & 17 & 5,8 & 16 & 5,0 & 8 & 3,5 & 12 & 4,0 \\
\hline 'Аүанос/п & 137 & 39,0 & 131 & 37,1 & 102 & 34,8 & 124 & 39,0 & 92 & 40,7 & 112 & 37,7 \\
\hline 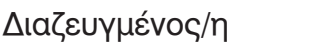 & 44 & 12,5 & 45 & 12,7 & 40 & 13,7 & 39 & 12,3 & 29 & 12,8 & 41 & 13,8 \\
\hline Xи́рос/а & 25 & 7,1 & 29 & 8,2 & 23 & 7,8 & 26 & 8,2 & 18 & 8.0 & 16 & 5,4 \\
\hline
\end{tabular}

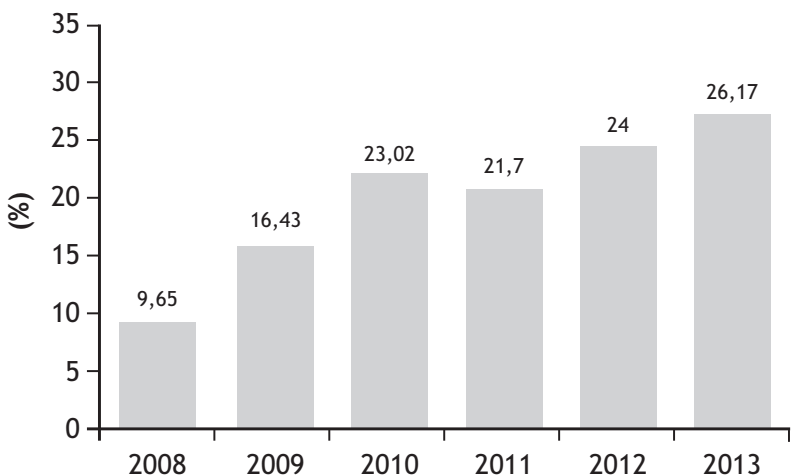

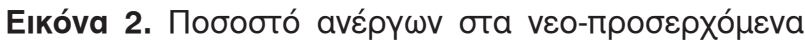

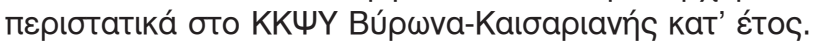

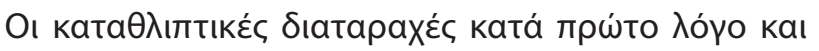

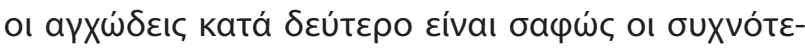

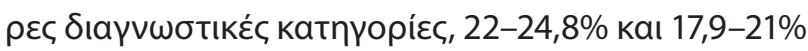

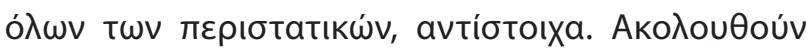

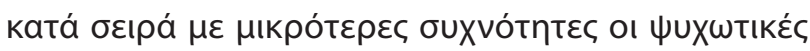

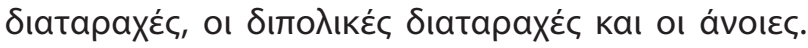

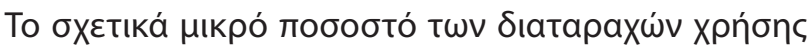

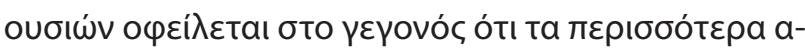

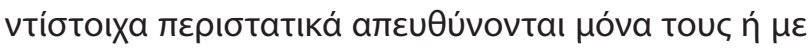

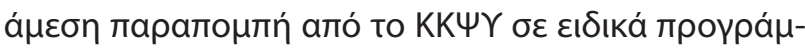

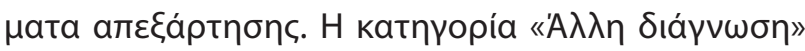

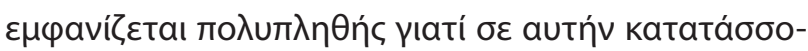

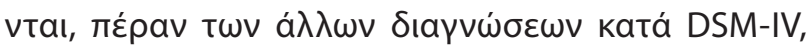

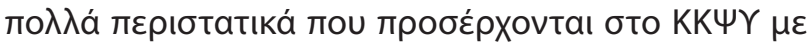

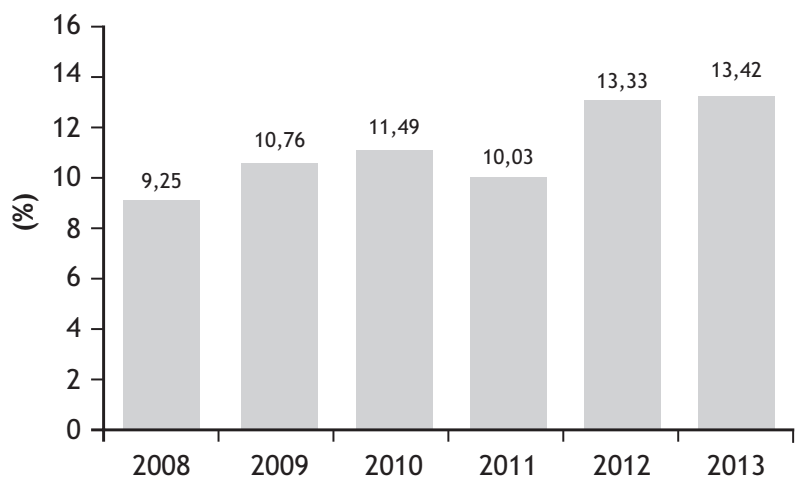

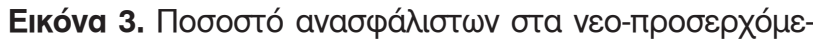

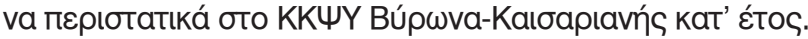

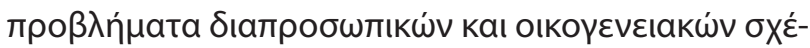

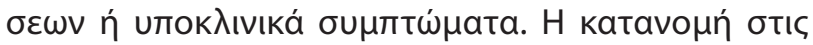

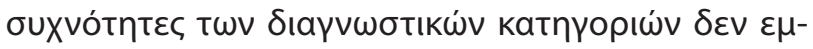

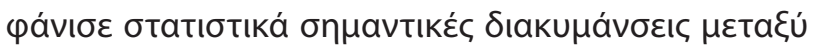

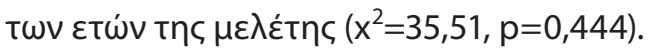

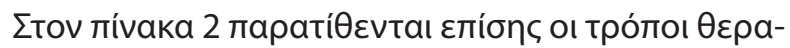

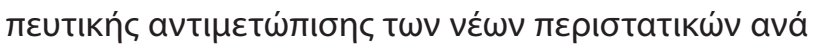

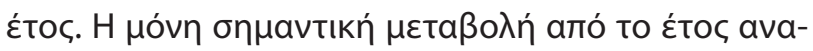

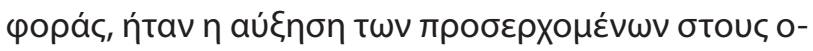

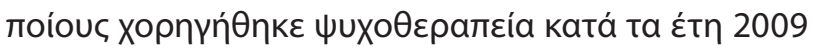
(OR=1,46, $\mathrm{p}=0,039), 2010$ (OR=1,49, $\mathrm{p}=0,038)$ кaı 2011 $(\mathrm{OR}=1,51, \mathrm{p}=0,028)$.

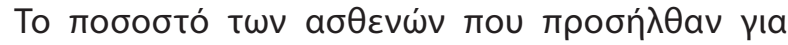

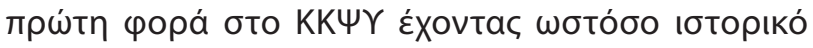




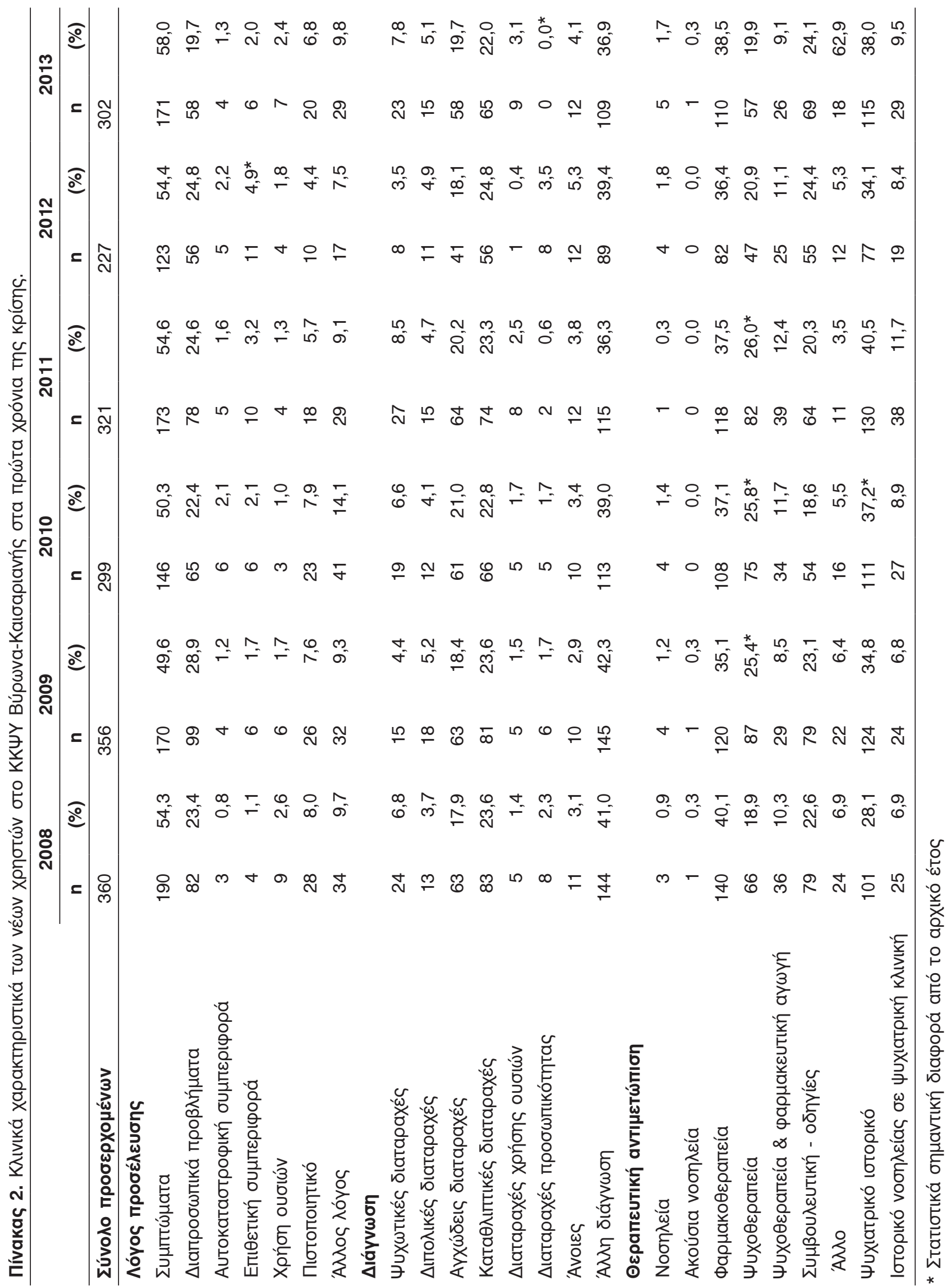




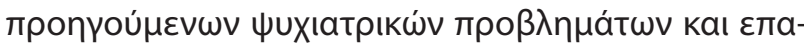

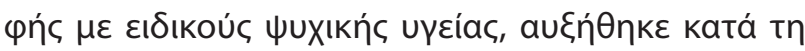

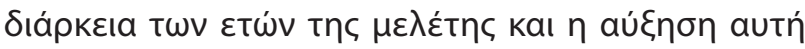

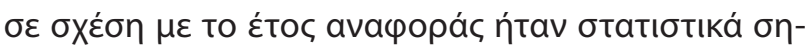

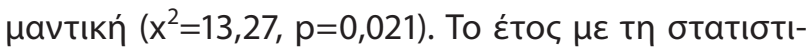

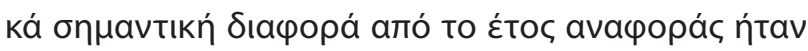

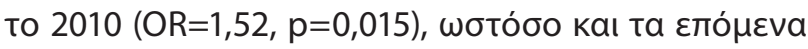

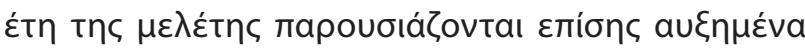

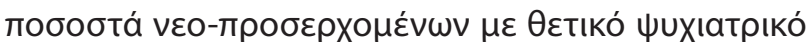

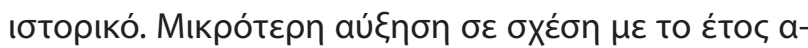

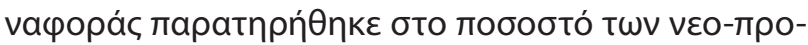

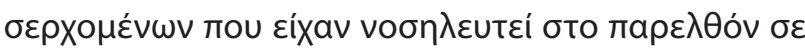

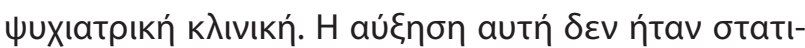

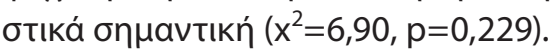

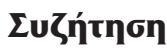

$\sum u ́ \mu \varphi \omega v a \mu \varepsilon$ ta a

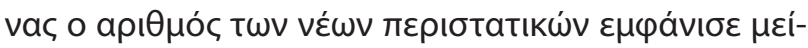

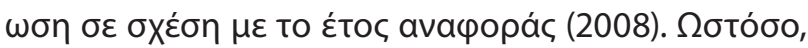

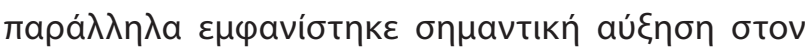

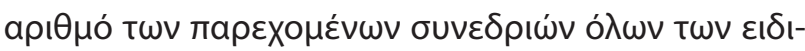

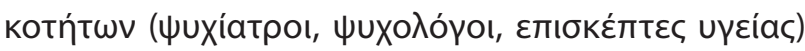

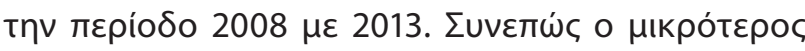

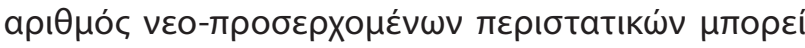

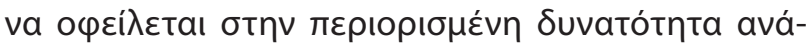

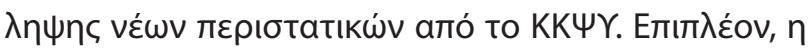

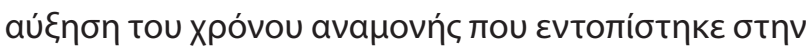

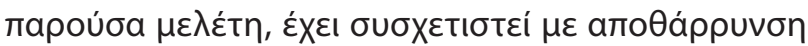

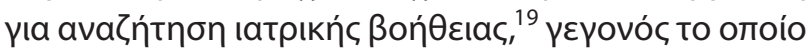

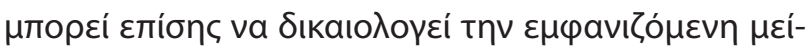

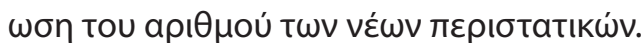

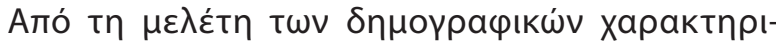

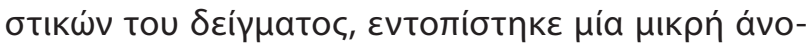

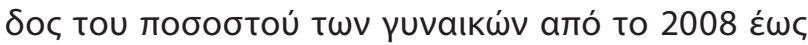

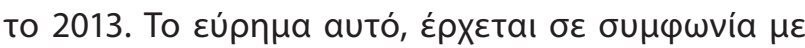

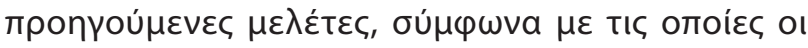

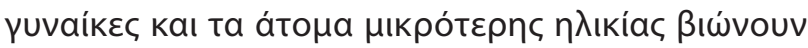

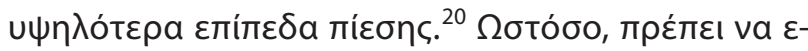

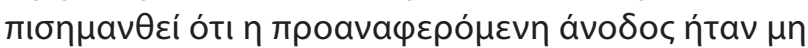

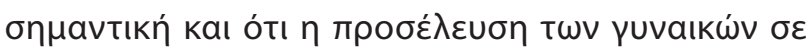

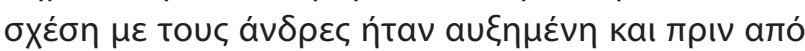

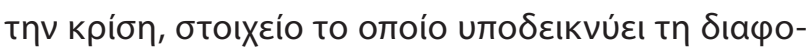

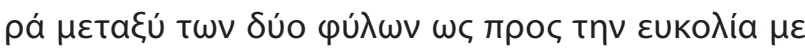

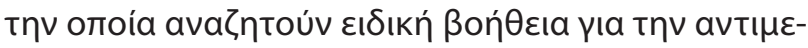

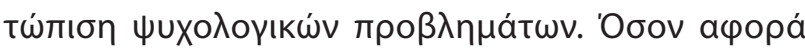

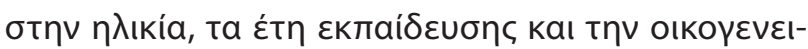

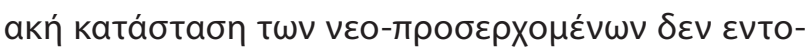

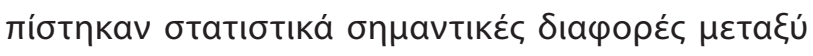

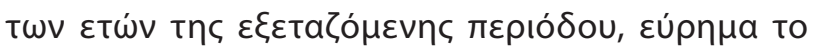

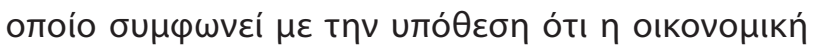

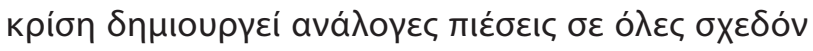

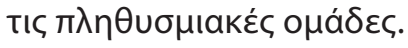

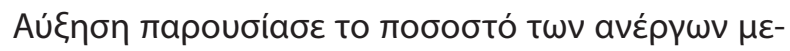

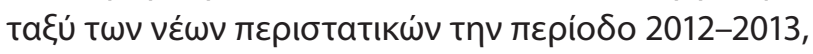

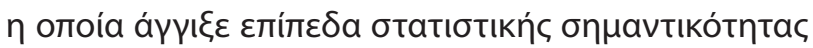

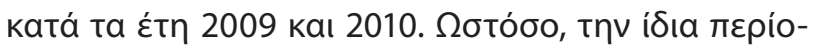

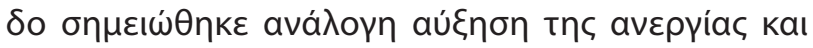

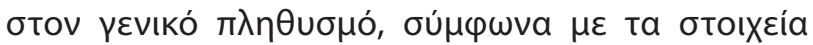

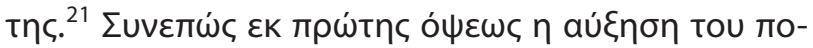

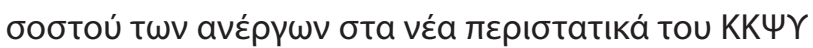

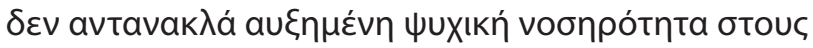

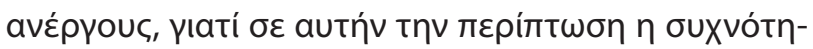

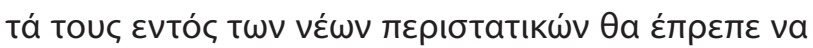

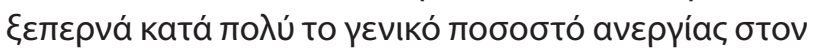

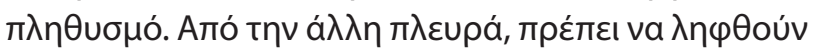
ито́

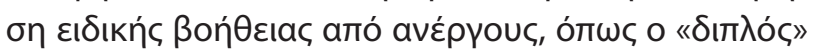

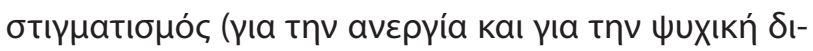

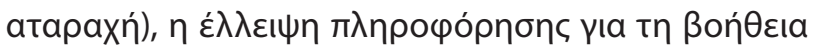

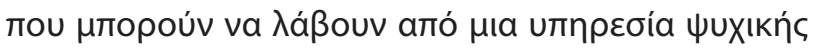

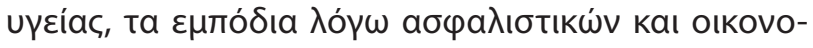

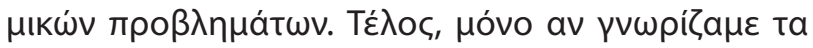

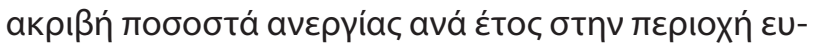

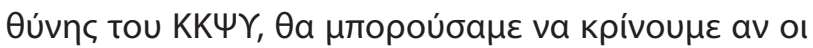

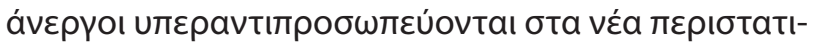

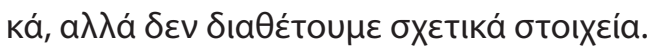

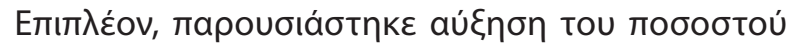

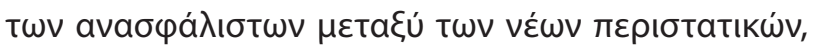

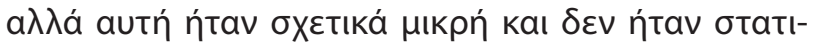

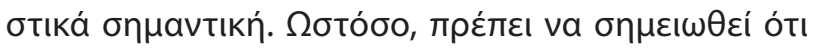

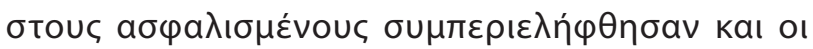

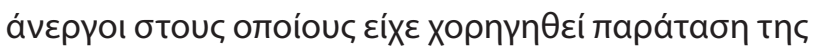

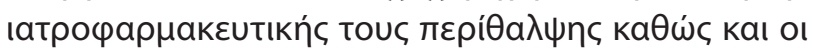

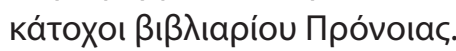

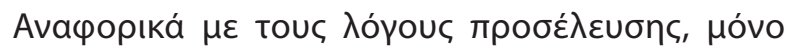

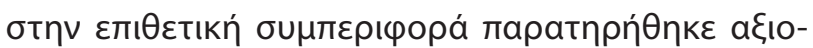

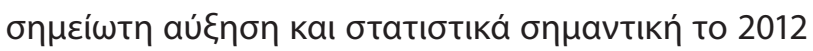

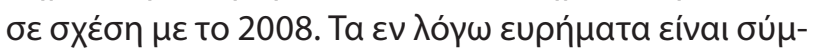

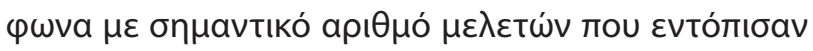

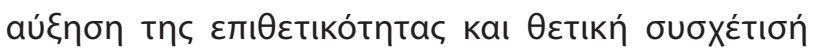

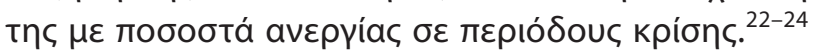
Епıт

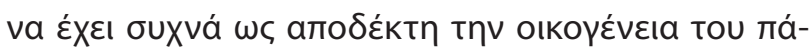

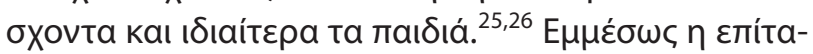




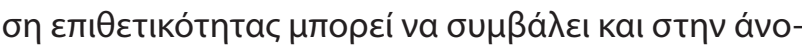

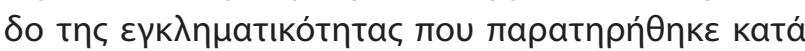

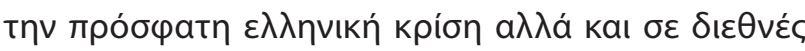

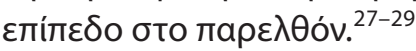

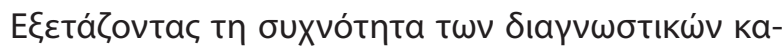

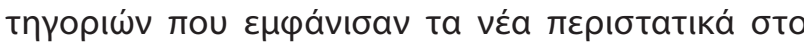

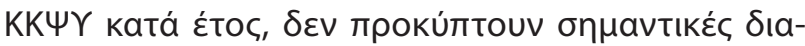

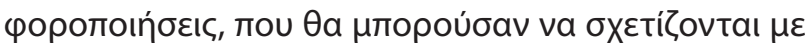

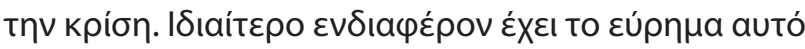

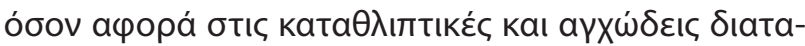

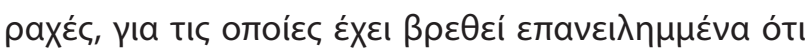

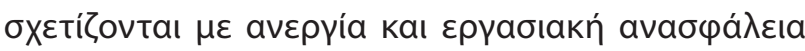

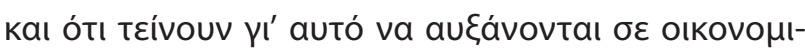

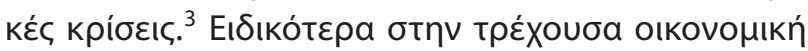

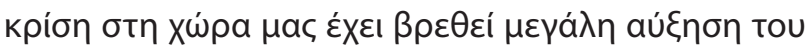

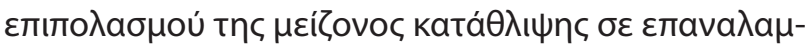

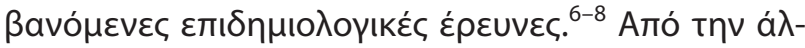

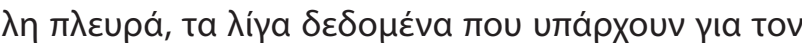

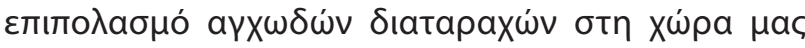

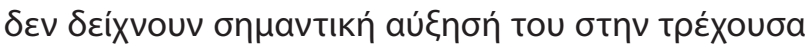

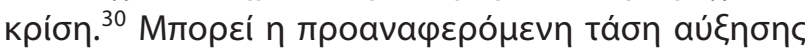

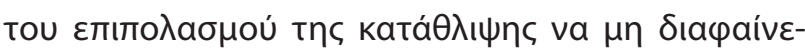

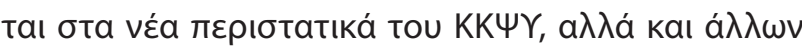

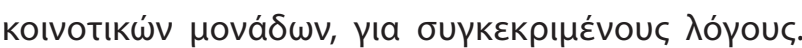

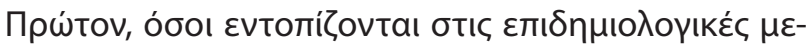

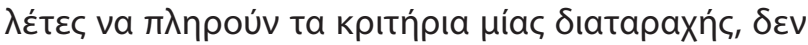

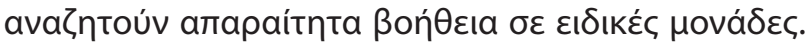

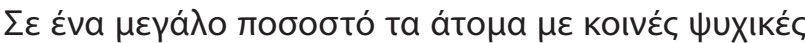

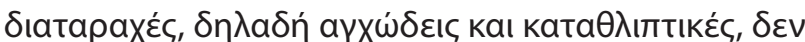

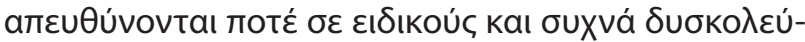

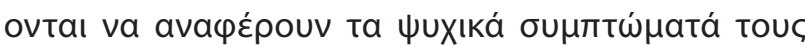

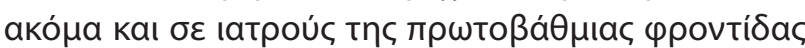

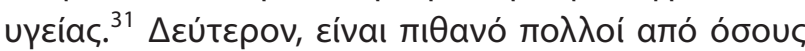

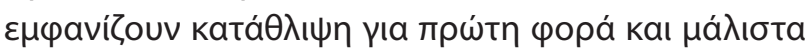

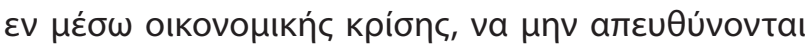

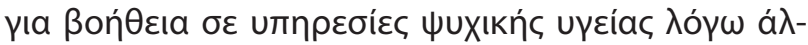

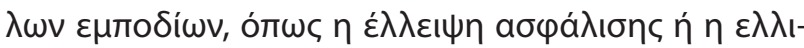

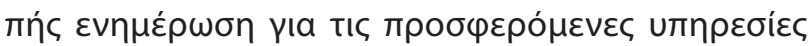

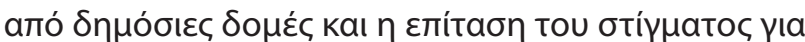

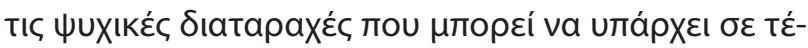

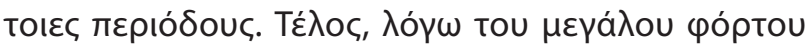

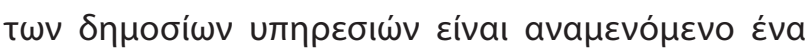

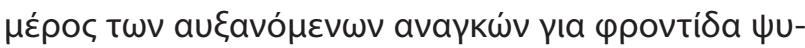

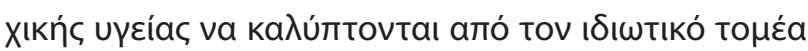

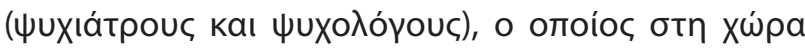

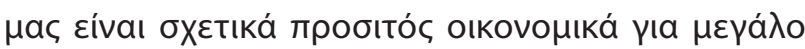

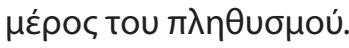

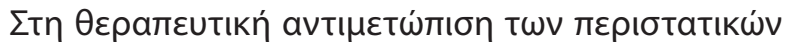

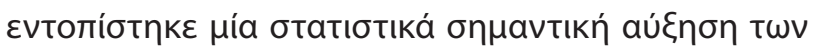

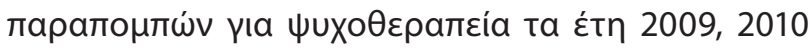

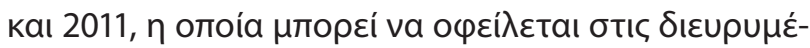

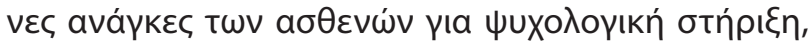

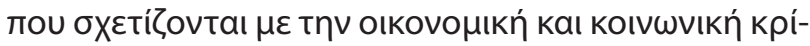

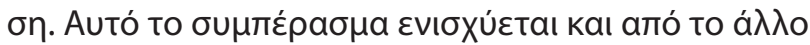

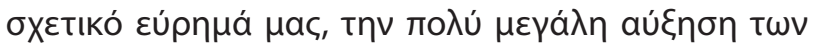

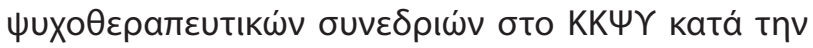

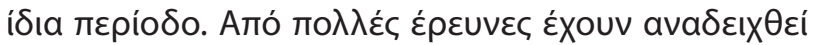

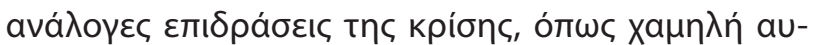

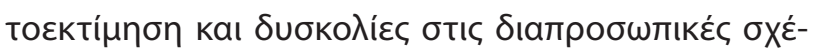

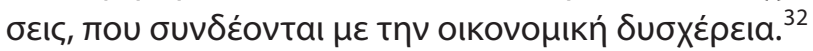

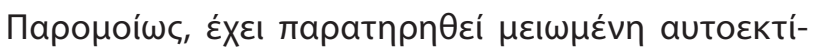

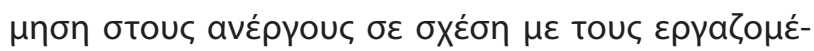
vouc. ${ }^{33}$

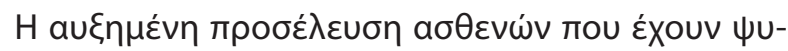

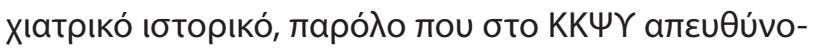

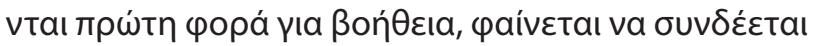

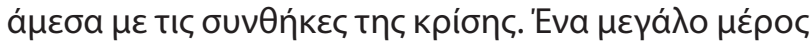

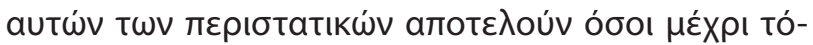

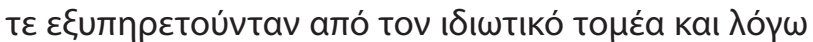

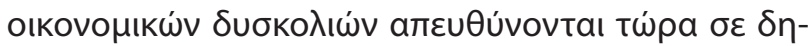

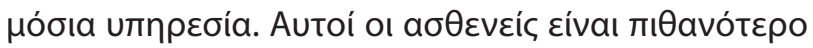

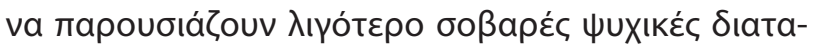

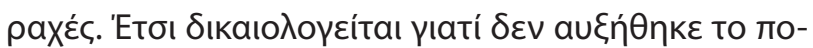

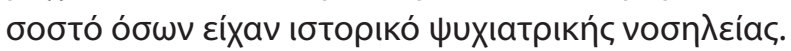

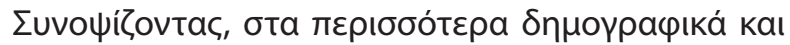

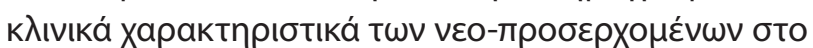

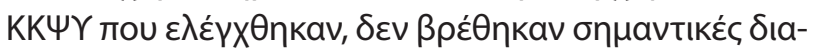

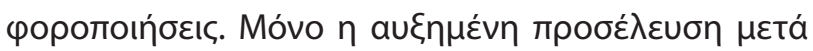

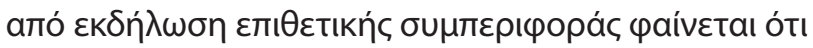

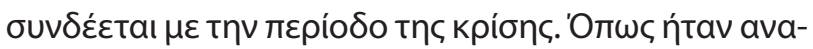

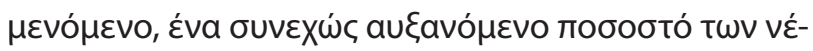

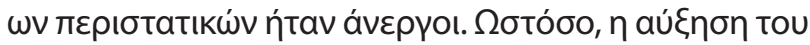

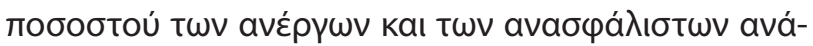

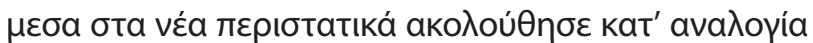

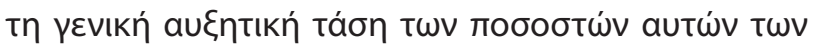

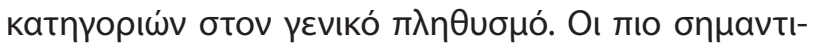

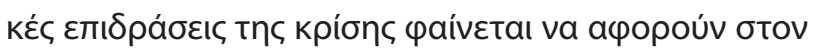

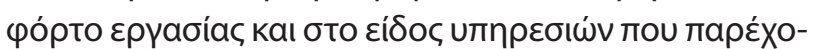

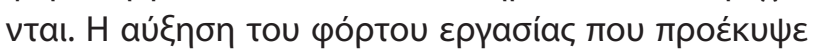

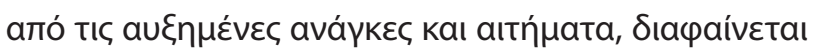

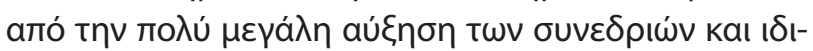

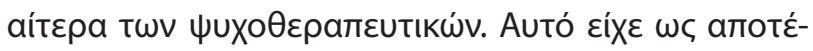

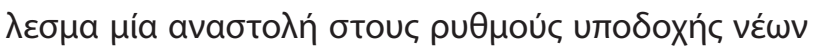

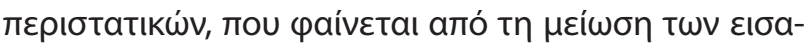




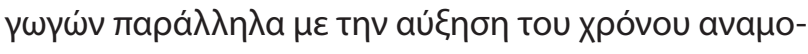

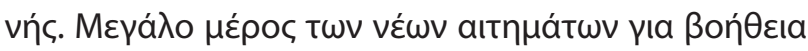

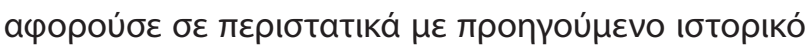

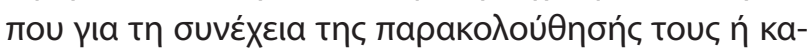

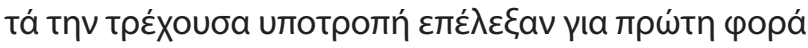

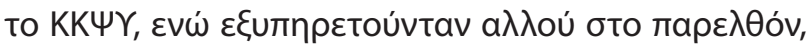

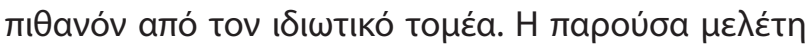

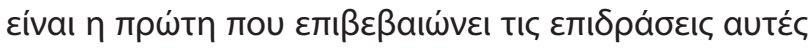

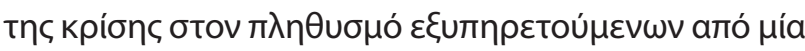

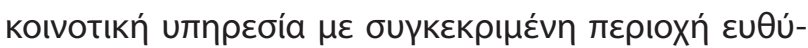

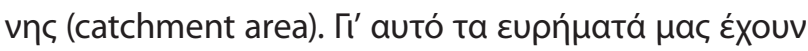

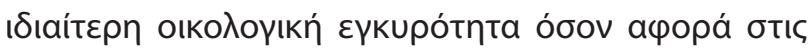

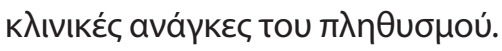

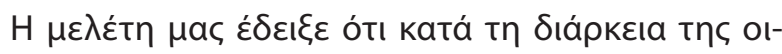

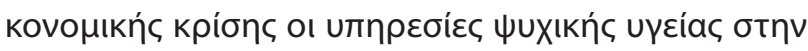

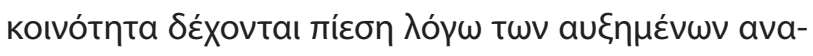

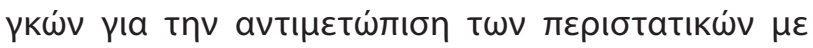

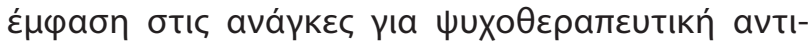

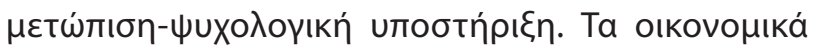

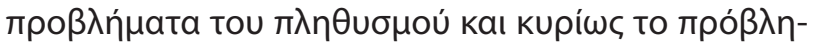

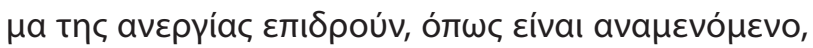

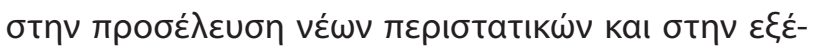

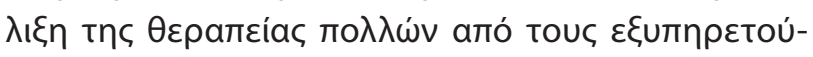

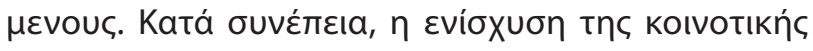

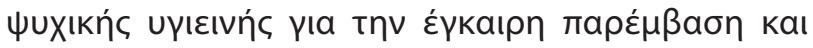

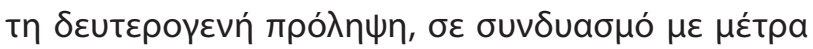

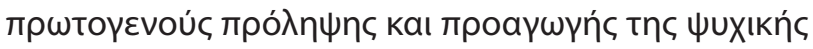

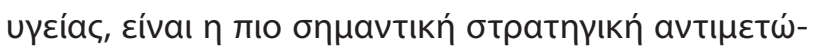

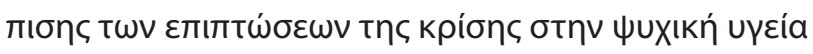
тou $\pi \lambda \eta \theta$

\title{
The impact of the recent financial crisis on the users' profile of a community mental health unit
}

\author{
K. Pikouli, ${ }^{1}$ G. Konstantakopoulos, ${ }^{1,2}$ P. Kalampaka Spilioti, ${ }^{1}$ \\ E. Fytrolaki, ${ }^{1}$ D. Ploumpidis, ${ }^{1}$ M. Economou ${ }^{1}$ \\ ${ }^{1}$ First Department of Psychiatry, Byron-Kessariani Community Mental Health Centre, \\ Athens University Medical School, Eginition Hospital, Athens, Greece, \\ ${ }^{2}$ Department of Psychosis Studies, Institute of Psychiatry, Psychology and Neuroscience, King's College London, UK
}

Psychiatriki 2019, 30:97-107

During previous financial crises as well as the recent global financial crisis, a strong impact of the crisis on the population mental health in many countries has been observed. Similarly, in Greece, a series of epidemiological studies pointed out the consequences of the economic crisis on the population mental health. However, there is limited data available, both in Greece and worldwide, regarding the impact of the economic crisis from the perspective of mental health services. The goal of the present study was to examine possible changes on the community mental health during the first years of the Greek financial crisis, as they are reflected on the operation of a community mental health unit with a specific catchment area within Athens (Byron and Kessariani). The demographic, social and clinical characteristics of adult users who were admitted for the first time at ByronKessariani Mental Health Community Center during the years 2008-2013 were analysed. The impact of the financial crisis on the workload of the center was also assessed during the same period. The sample of the study consisted of 1865 adult users and the data was collected with the use of an ad hoc structured questionnaire as well as from the users' case files. No significant differentiation on the number of clients admitted to the center per year after the beginning of the financial crisis was 
found. However, it is possible that an upper limit has been reached on the center's capacity to admit new clients, i.e. a ceiling effect, as it is shown from the increased number of provided sessions per year as well as from the increase in the mean waiting time for the intake of new patients during the same period. A constant increase in the number of women among the new clients of ByronKessariani Mental Health Community Center was found, but no significant differentiations were detected during the study period. Moreover, the study showed an upward trend in aggressive behavior as a reason for admission, a significant and continuous increase in the rate of unemployed individuals among the new clients, as well as a statistically significant increase in the number of referrals for psychotherapy during the study period. There was also an increase in the number of patients who had psychiatric history, even though they were admitted to Byron-Kessariani Mental Health Community Center for the first time. No significant differentiations were found in the remaining users' demographic and clinical characteristics assessed. Our study showed that during the crisis community mental health services are under pressure due to the increased needs of patients, especially the needs for psychotherapeutic intervention and psychological support. The increased unemployment rates affect the influx of new patients as well as the therapeutic management of many users. Reinforcement of the community mental health service network is an important strategy against the consequences of the crisis on the population mental health.

Key words: Financial crisis, community mental health, patient demands, unemployment, aggressive behavior.

\section{BıßAtoypacpía}

1. Marmot MG, Bell R. How will the financial crisis affect health? BMJ 2009, 338:b1314, doi: 10.1136/bmj.b1314

2. Uutela A. Economic crisis and mental health. Curr Opin Psychiatry 2010, 23:127-130, doi: 10.1097/YCO.0b013e328336657d

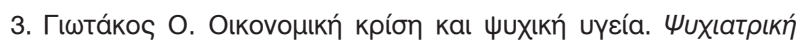
2010, 21:195-204, PMID: 21914618

4. Parmar D, Stavropoulou C, loannidis JPA. Health outcomes during the 2008 financial crisis in Europe: systematic literature review BMJ 2016, 354:i4588, doi:10.1136/bmj.i4588

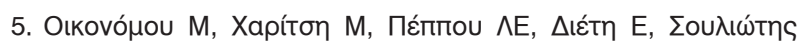

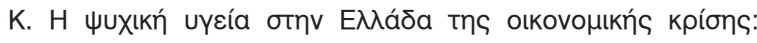

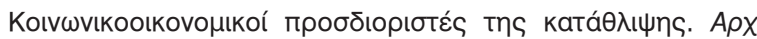

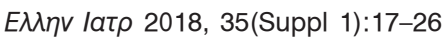

6. Madianos M, Economou M, Alexiou T, Stefanis C. Depression and economic hardship across Greece in 2008 and 2009: two cross-sectional surveys nationwide. Soc Psychiatry Psychiatr Epidemiol 2011, 46:943-952, doi: 10.1007/s00127-010-0265-4

7. Economou M, Madianos M, Peppou LE, Patelakis A, Stefanis CN. Major depression in the Era of economic crisis: A replication of a cross-sectional study across Greece. J Affect Disord 2013, 145:308-314, doi: 10.1016/j.jad.2012.08.008

8. Economou M, Angelopoulos E, Peppou LE, Souliotis K, Tzavara $C$ et al. Enduring financial crisis in Greece: prevalence and correlates of major depression and suicidality. Soc Psychiatry Psychiatr Epidemiol 2016, 51:1015-1024, doi: 10.1007/s00127016-1238-z

9. Kontaxakis B, Papaslanis T, Havaki-Kontaxaki M, Tsouvelas G, Giotakos O, Papadimitriou G. Suicide in Greece: 2001-2011. Psychiatriki 2013, 24:170-174, PMID: 24185083
10. Fountoulakis KN, Koupidis SA, Grammatikopoulos IA, Theodorakis PN. First reliable data suggest a possible increase in suicides in Greece. BMJ 2013, 347, f4900, doi: 10.1136/bmj.f4900

11. Economou M, Madianos M, Theleritis C, Peppou LE, Stefanis CN. Increased suicidality amid economic crisis in Greece. Lancet 2011, 378:1459, doi: 10.1016/S0140-6736(11)61638-3

12. Economou M, Madianos M, Peppou LE, Theleritis C, Patelakis A, Stefanis C. Suicidal ideation and reported suicide attempts in Greece during the economic crisis. World Psychiatry 2013, 12:53-59, doi: 10.1002/wps.20016

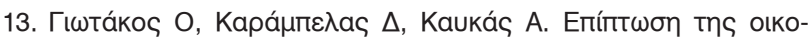

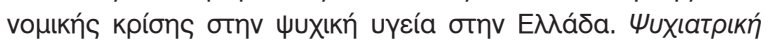
2011, 22:109-119, PMID: 21888184

14. Fountoulakis KN, Savopoulos C, Apostolopoulou M, Dampali R, Zaggelidou E, Karlafti E et al. Rate of suicide and suicide attempts and their relationship to unemployment in Thessaloniki Greece (2000-2012). J Affect Disord 2015, 174:131-136, doi: 10.1016/j.jad.2014.11.047

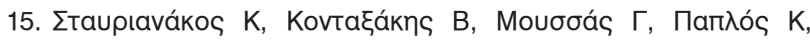

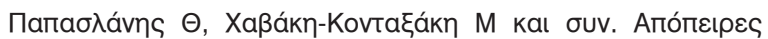

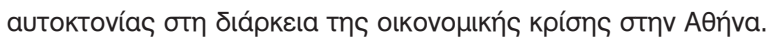
UuХıатрıќ́ 2014, 25:104-110, PMID: 25035179

16. World Health Organization. The financial crisis and global health. WHO, Geneva, 2001. Available at: http://www.who.int/mediacentre/events/meetings/2009_financial_crisis_report_en_.pdf

17. Laliotis I, Ioannidis JPA, Stavropoulou C. Total and cause-specific mortality before compared with after the onset of the Greek economic crisis: an interrupted time-series analysis. Lancet Public Health 2016, 1:e56-e65, doi: 10.1016/S2468-2667(16)30018-4

18. American Psychiatric Association. Diagnostic and statistical manual of mental disorders, Fourth Edition, Text Revision. American 
Psychiatric Association, Washington DC, 1994, doi: 10.1007/ springerreference_179660

19. Kontaxakis VP, Havaki-Kontaxaki BJ. Consequences of major economic crises on citizen's physical and mental health. Psychiatriki 2012, 23:105-108, PMID: 22796908

20. Kalogeropoulou M, Papathanasopoulou E. The impact of the economic crisis on the mental health of nurses: A pilot study Arch Hellen Med 2013, 30:587-594

21. Hellenic Statistical Authority (ELSTAT). Labour force (Monthly data). Available from: http://www.statistics.gr/en/statistics/-/publication/SJO02/-(Accessed 6 August 2018)

22. Fischer P, Greitemeyer T, Frey D. Unemployment and aggression: The moderating role of self-awareness on the effect of unemployment on aggression. Aggress Behav 2008, 34:34-45, doi: 10.1002/ab.20218

23. Kivimaki M, Elovainio M, Kokko K, Pulkkinen L, Kortteinnen M, Tuomikoski H. Hostility, unemployment and health status: testing three theoretical models. Soc Sci Med 2003, 56:2139-2152, doi: 10.1016/S0277-9536(02)00219-8

24. Nordin M, Almın D. Long-term unemployment and violent crime. Empir Econ 2017, 52:1-29, doi: 10.1007/s00181-016-1068-6

25. Catalano RA, Lind SL, Rosenblatt AB, Attkisson, CC. Unemployment and foster home placements: estimating the net effect of provocation and inhibition. Am J Public Health 1999, 89:851-855, PMID: 10358674

26. O' Dowd A. Recession could increase alcohol and drug addiction. BMJ 2009, 339:b3361, doi: 10.1136/bmj.b3361

27. Tsouvelas G, Kontaxakis V, Giotakos O, Konstantakopoulos G, Kontaxaki M-I, Papaslanis Th. Financial crisis and criminality in Greece. 2008 vs 2011. Psychiatriki 2016, 27:54-57, PMID: 27110884

28. Tsouvelas G, Konstantakopoulos G, Vakirtzis A, Giotakos O, Papaslanis Th, Kontaxakis V, Criminality in Greece during the years of financial crisis: 2008-2014. Psychiatriki 2018, 29:19-24, doi: 10.22365/jpsych.2018.291.19

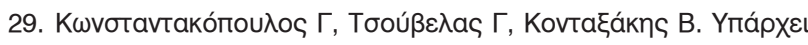

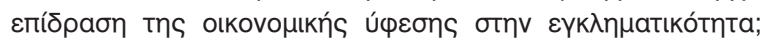

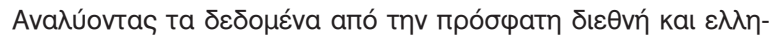

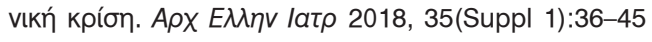

30. Oıкоvó

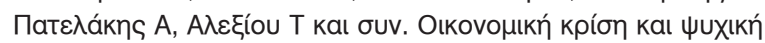

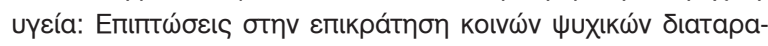

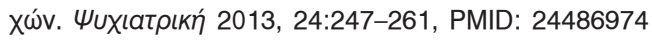

31. Robinson JW, Roter DL. Psychosocial problem disclosure by primary care patients. Soc Sci Med 1999, 48:1353-1362, PMID: 10369436

32. Fitch C, Hamilton S, Bassett P, Davey R. The relationship between personal debt and mental health: a systematic review. Med HIth Rev 2011, 16:153-166, doi: 10.1108/13619321111202313

33. Paul K, Moser K. Unemployment impairs mental health: Metaanalyses. J Vocat Behav 2009, 74:264-282, doi: 10.1016/j.jvb. 2009.01.001

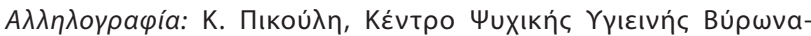

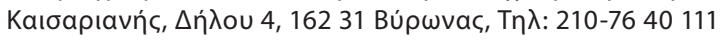
e-mail:katpikouli@yahoo.gr 


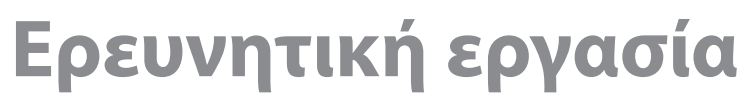 Research article
}

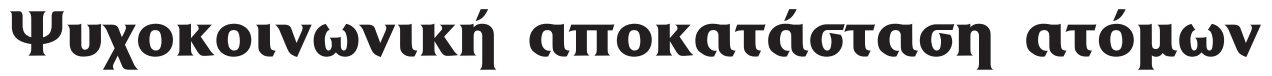

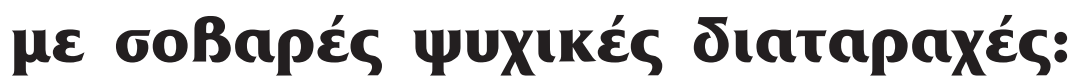

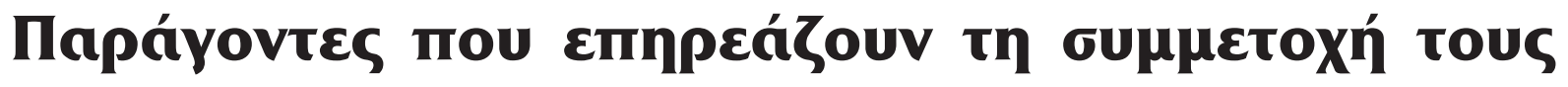

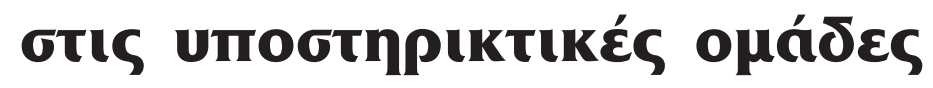

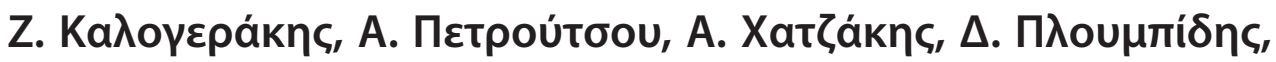

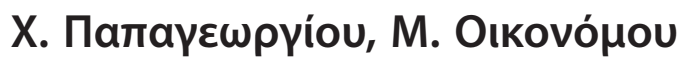

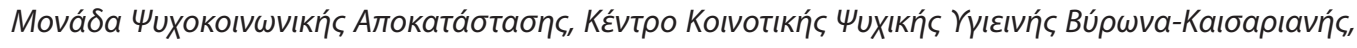

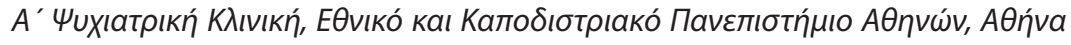

Uuxıатрıкń 2019, 30:108-119

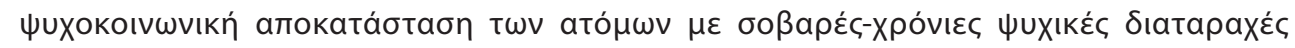

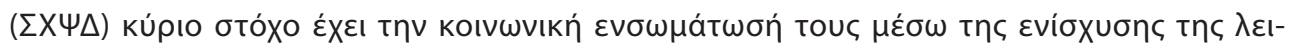

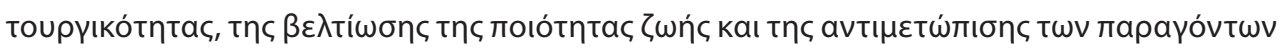

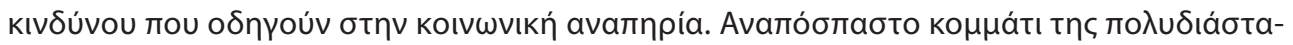

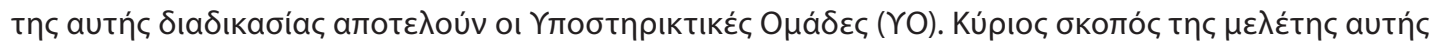

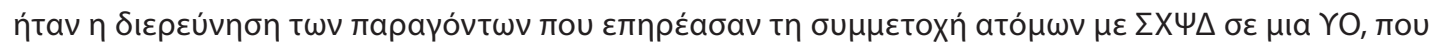

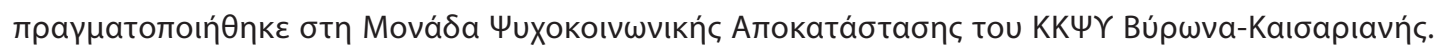

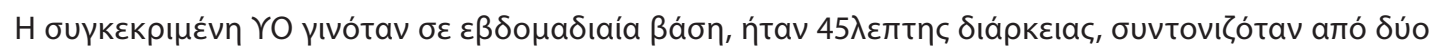

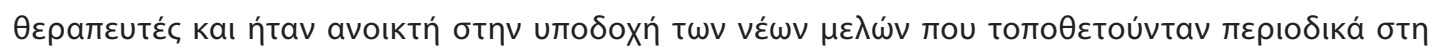

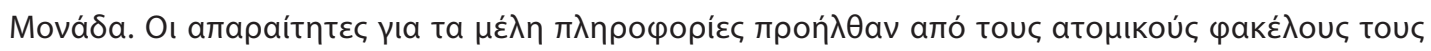

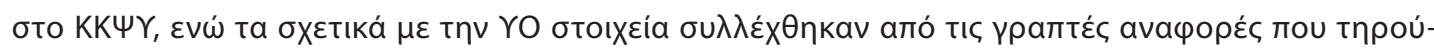

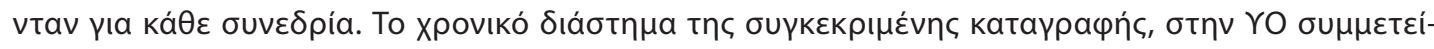

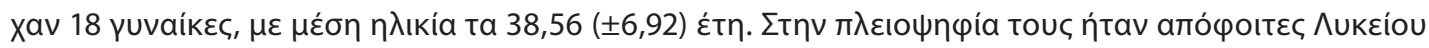

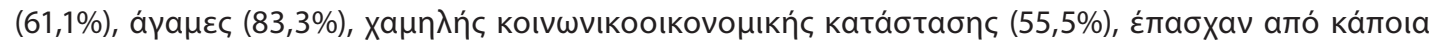

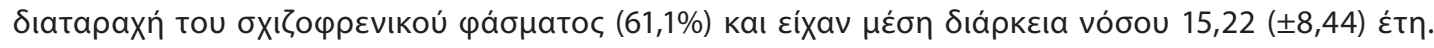

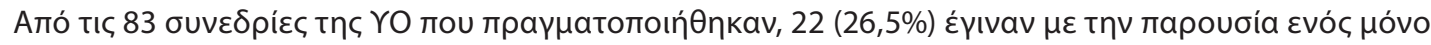




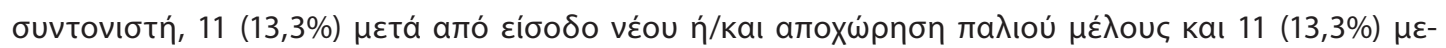

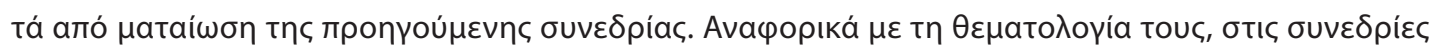

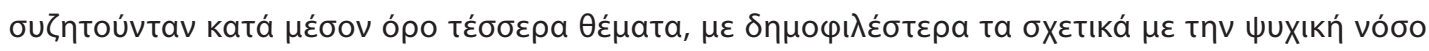

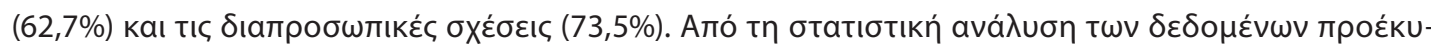

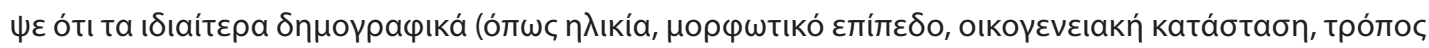

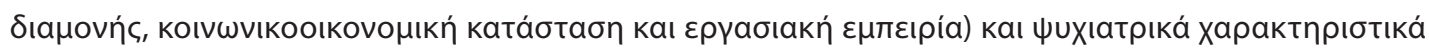

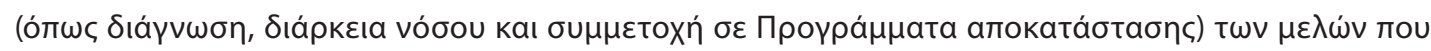

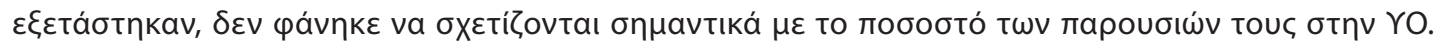

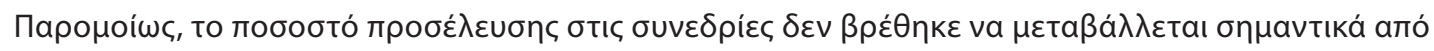

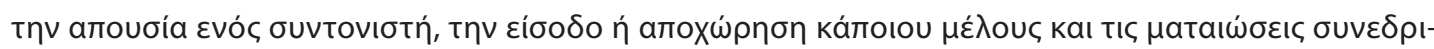

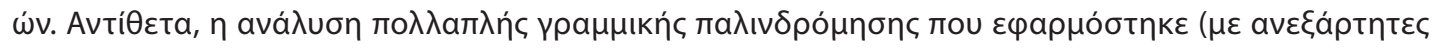

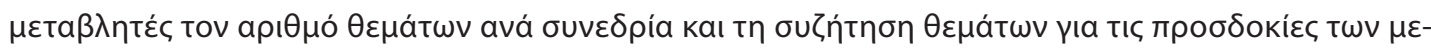

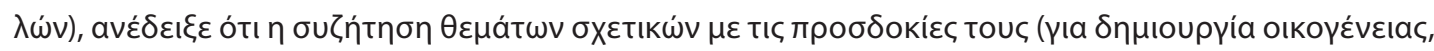

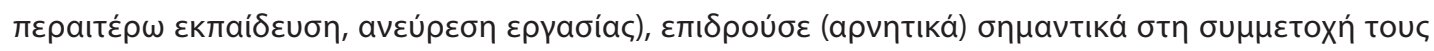

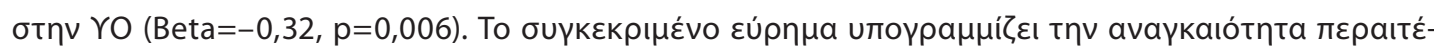

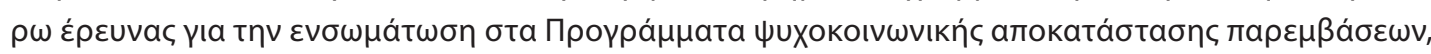

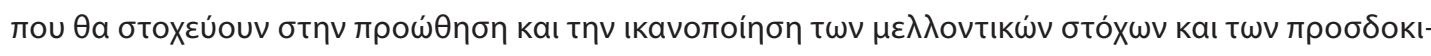
$\omega \dot{v} \tau \omega v$ ató $\mu \omega v \mu \varepsilon \Sigma X \Psi \Delta$.

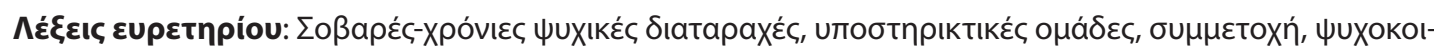

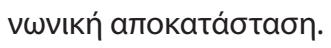

\section{Eıбaywyń}

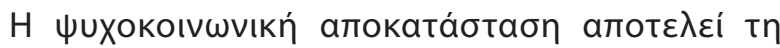

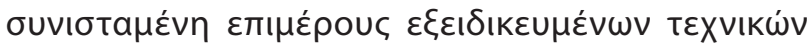

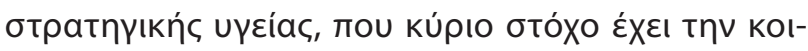

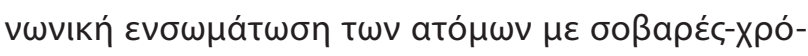

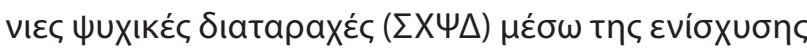

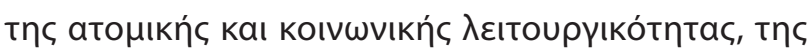

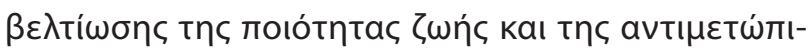

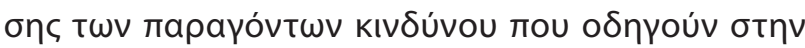

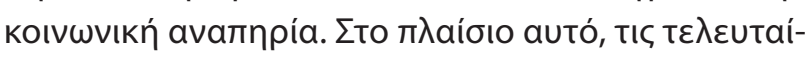

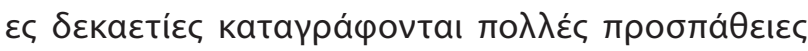

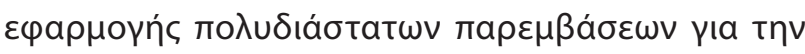

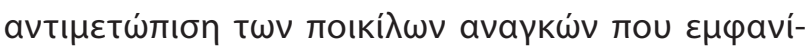

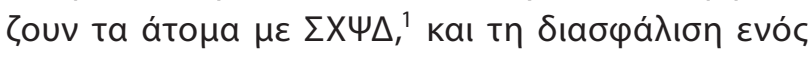

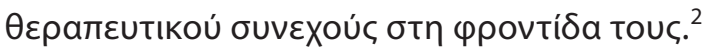

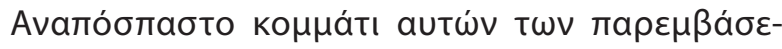

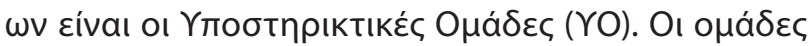

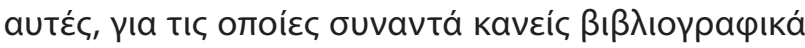

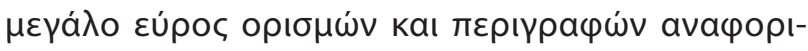

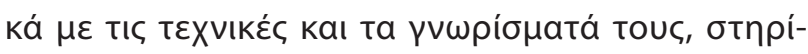

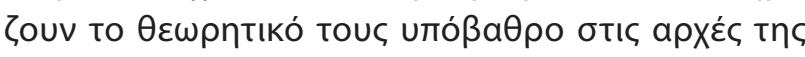

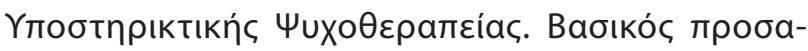

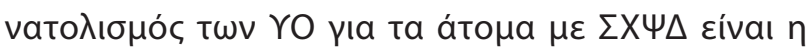

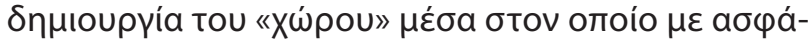

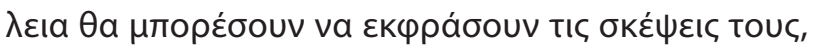

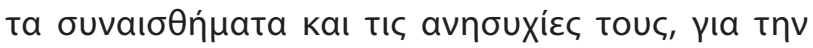

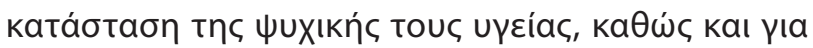

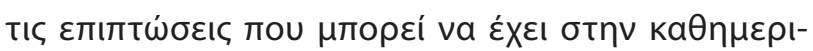

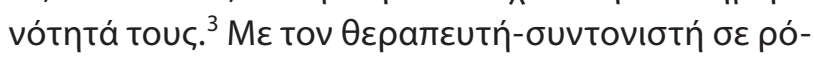

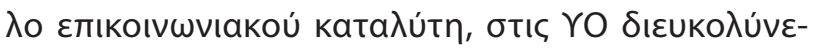

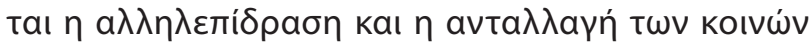

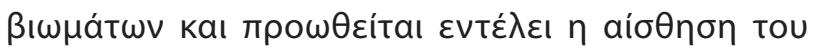

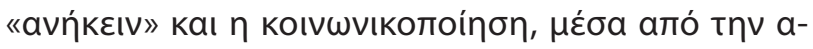

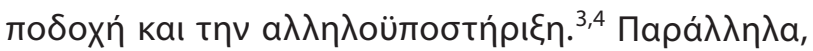

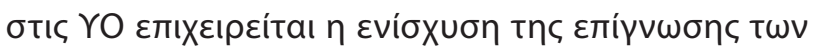

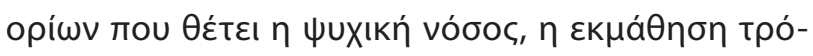

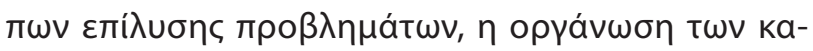

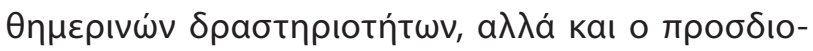

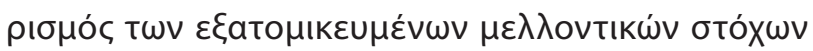
kal $\sigma x \varepsilon \delta i ́ \omega v .^{5}$

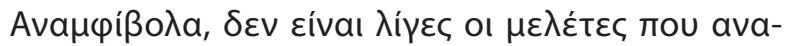

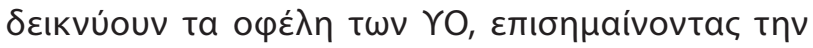

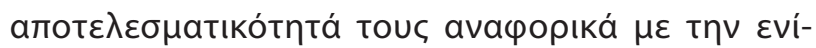

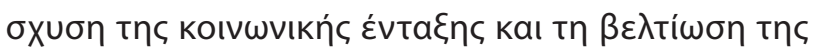

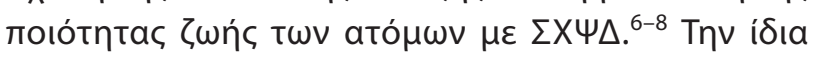

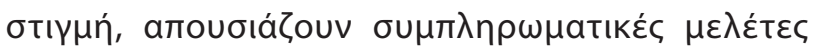

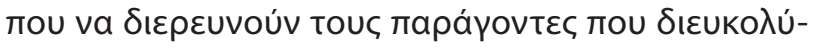




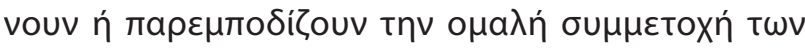

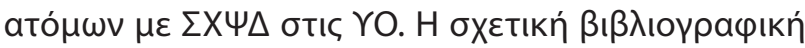

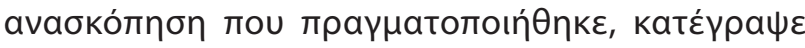

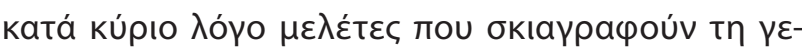

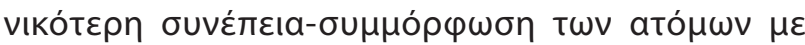

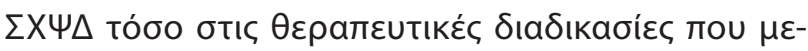

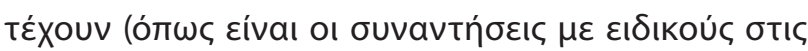

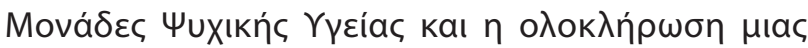

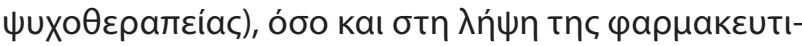

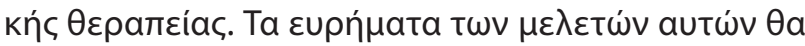

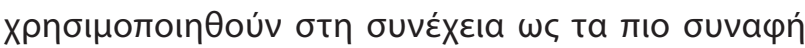

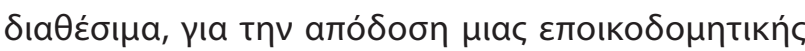

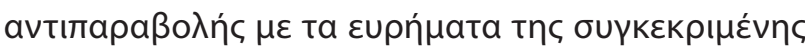

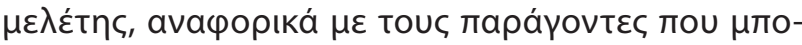

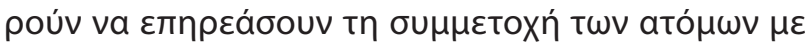
$\Sigma X \Psi \Delta$ бтІ५ $Y O$.

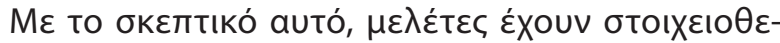

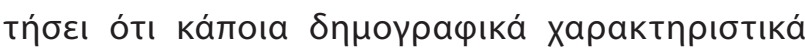

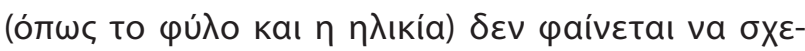

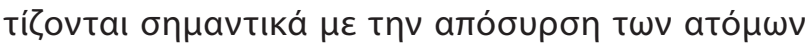

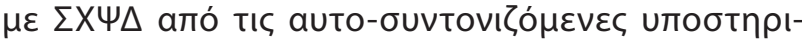

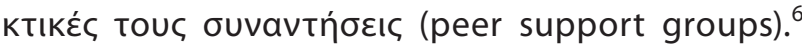

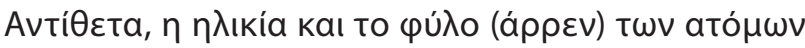

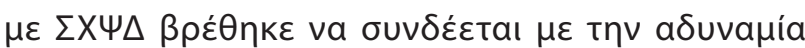

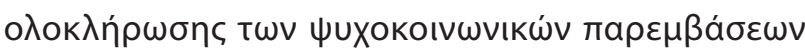

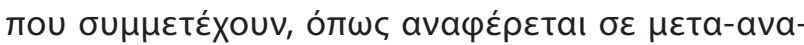

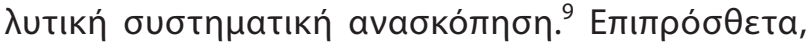

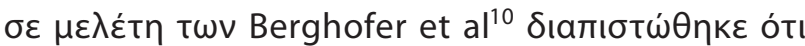

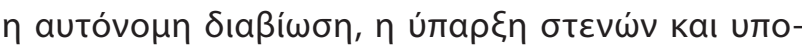

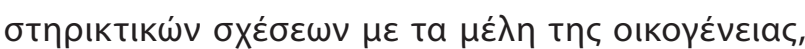

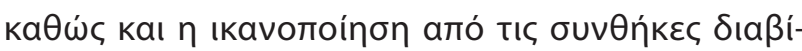

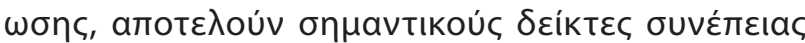

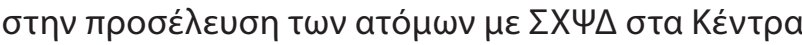

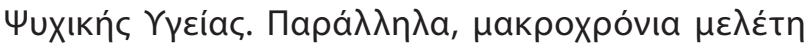

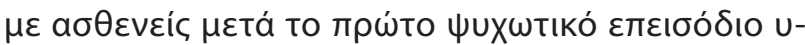

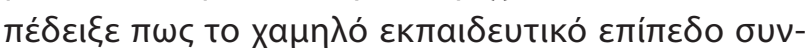

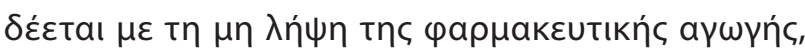

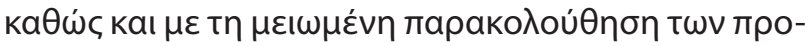

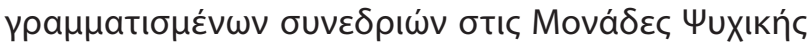

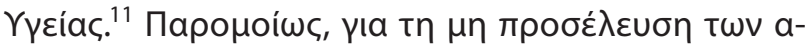

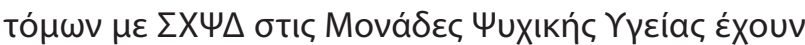

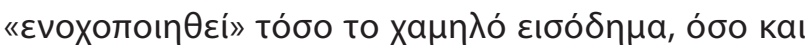
$\eta$ avepyía. ${ }^{10,12}$

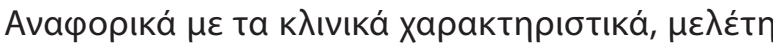

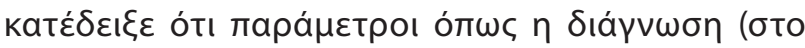

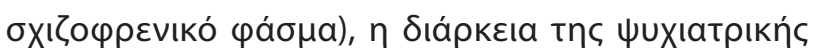

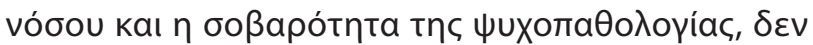

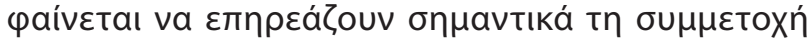

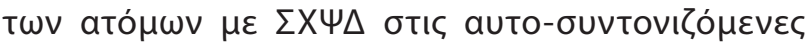

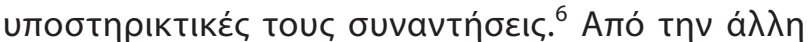

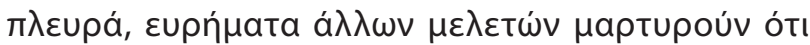

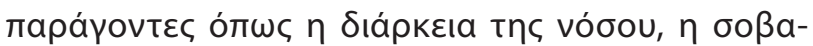

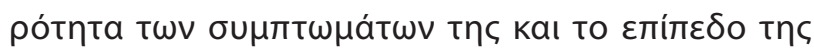

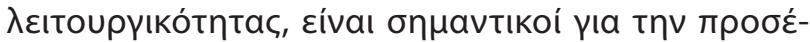

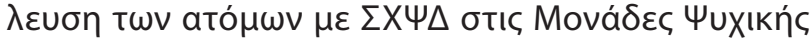

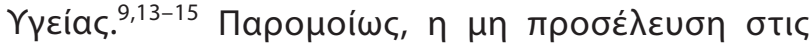

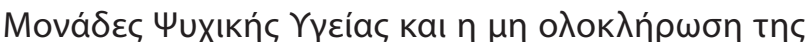

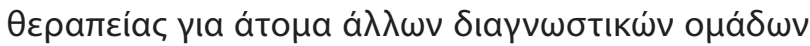

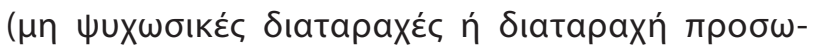

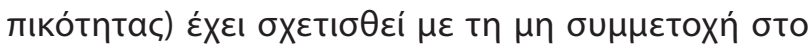

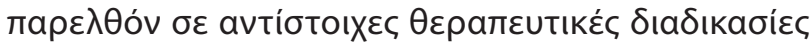

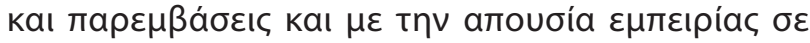

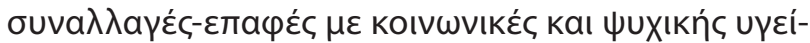

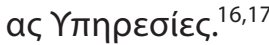

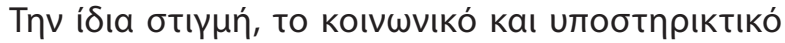

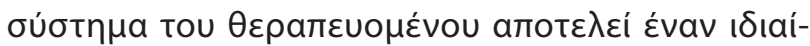

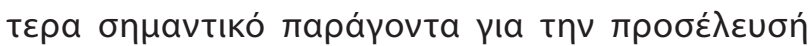

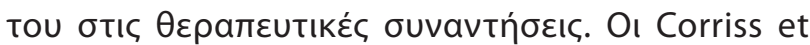

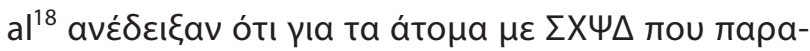

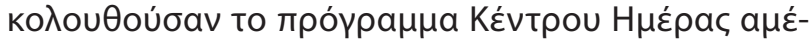

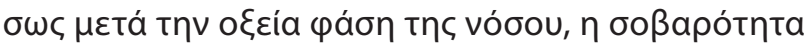

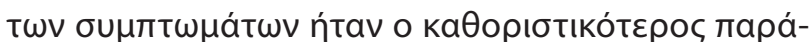

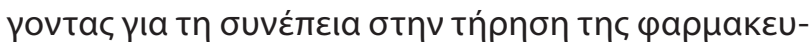

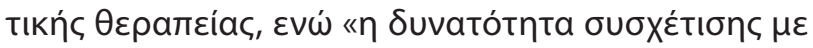

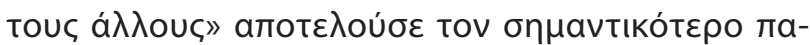

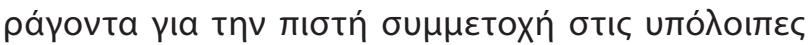

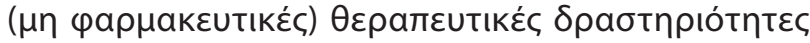

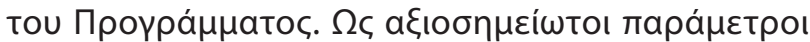

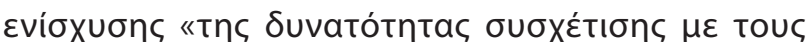

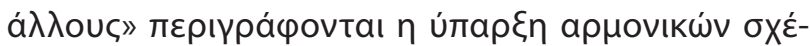

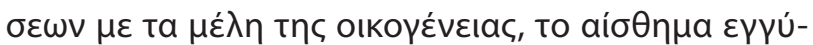

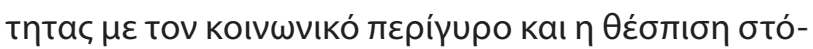

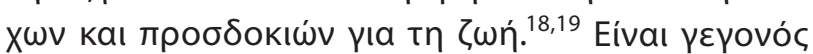

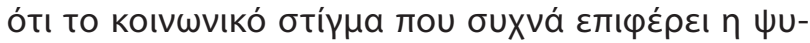

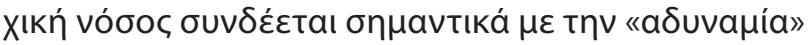

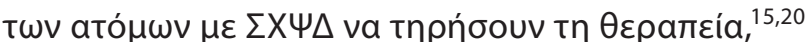

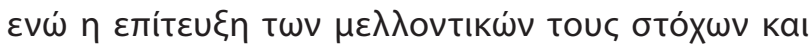

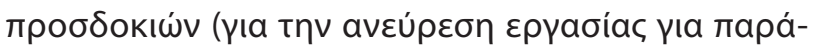

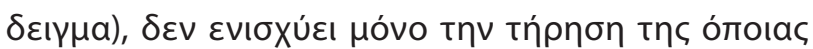

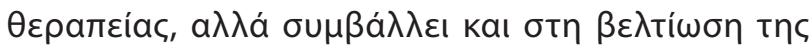

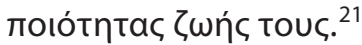




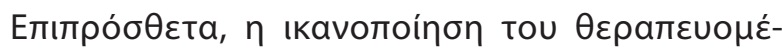

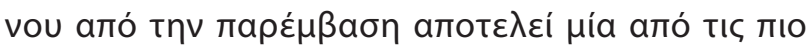

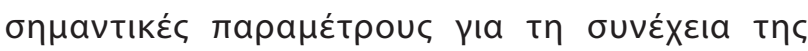

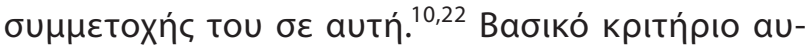

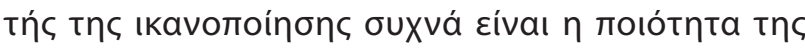

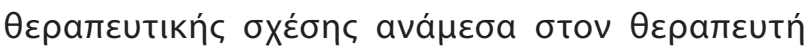

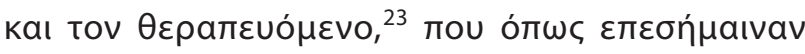

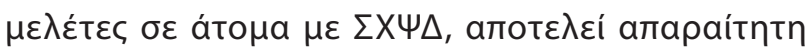

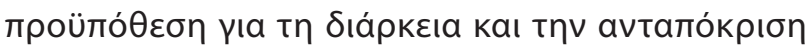

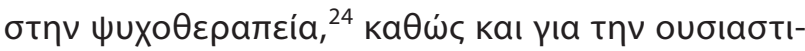

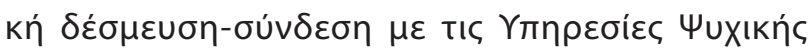

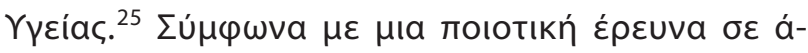

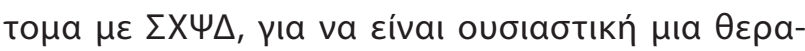

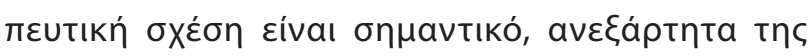

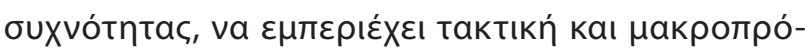

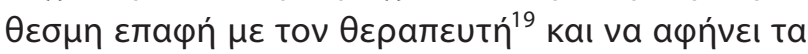

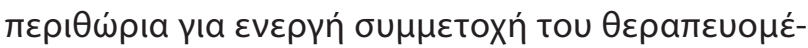

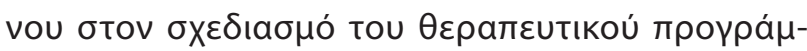

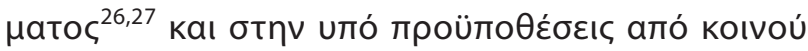

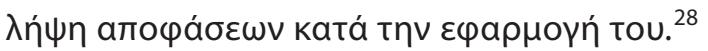

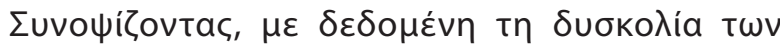

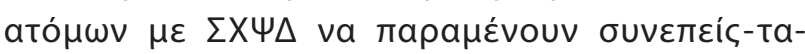

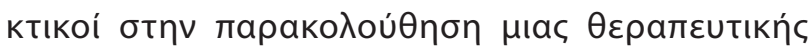

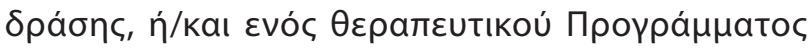

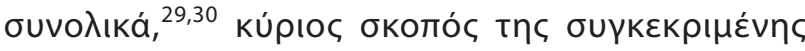

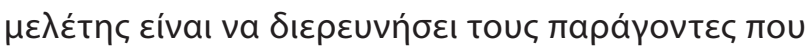

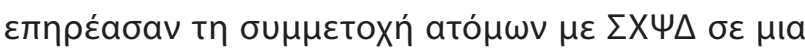

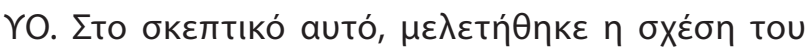

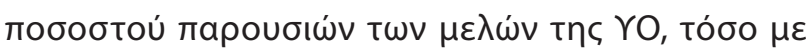

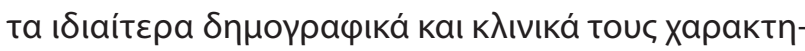

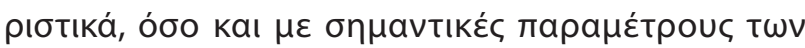

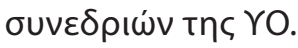

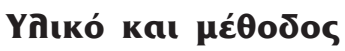

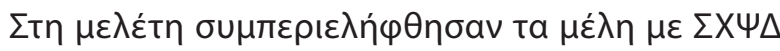

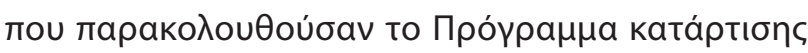

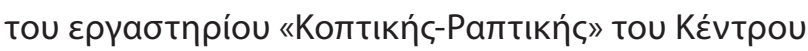

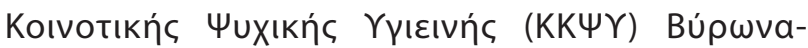

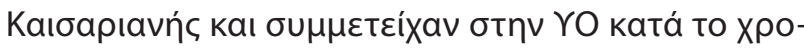

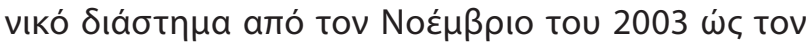

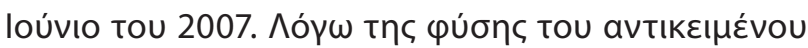

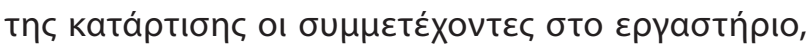

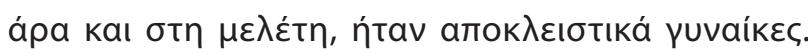

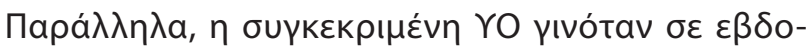

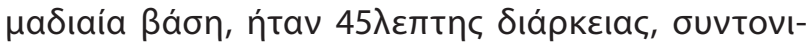

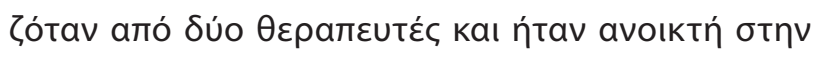

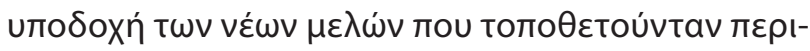

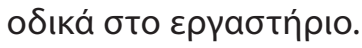

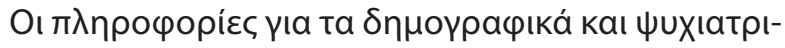

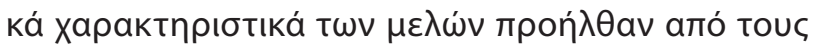

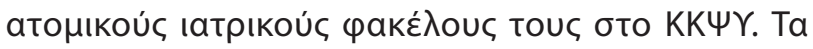

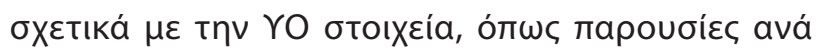

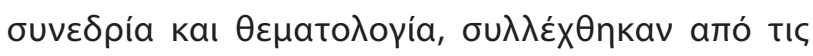

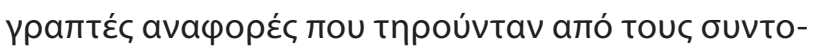

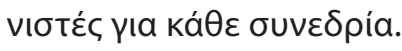

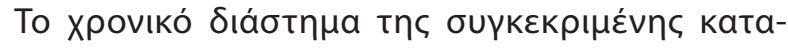

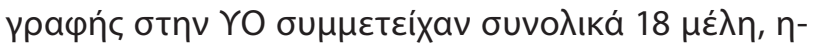

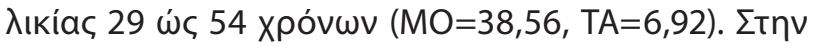

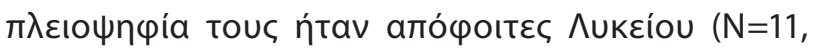

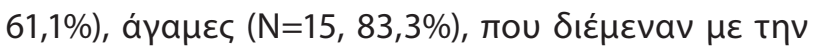

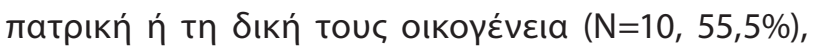

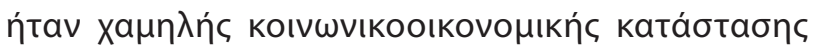

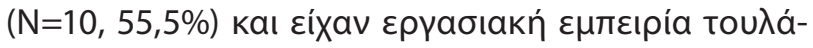

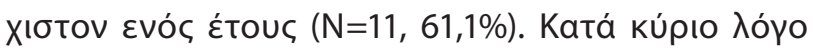

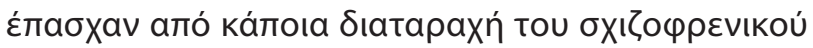

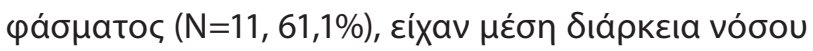

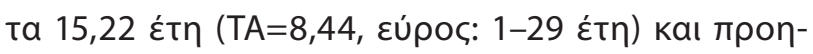

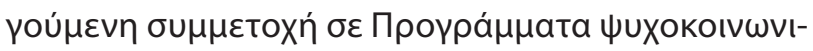

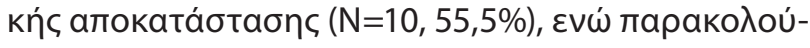

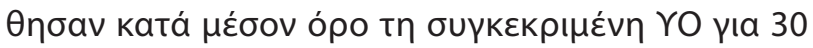

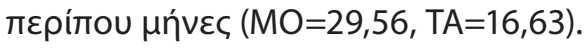

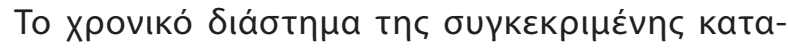

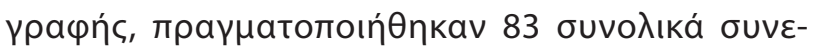

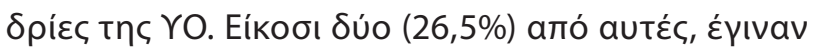

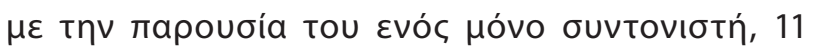

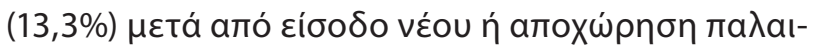

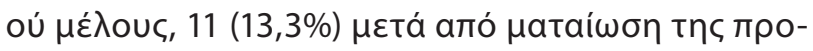

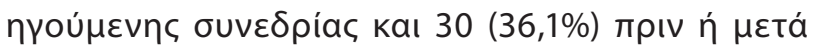

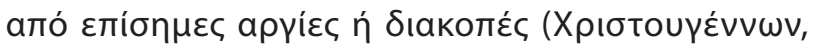

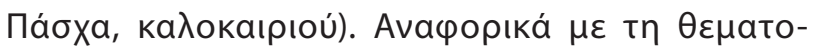

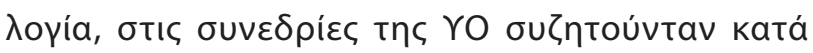

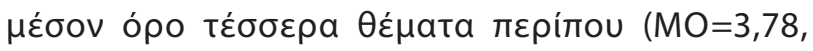

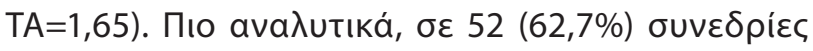

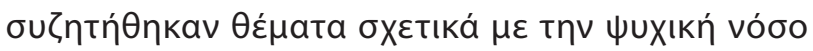

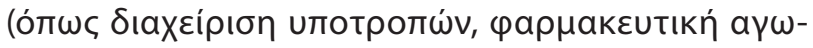

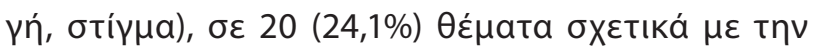

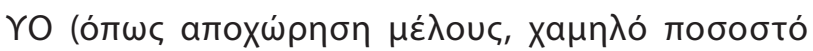

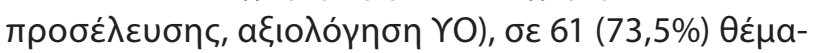

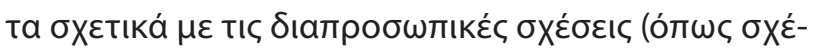

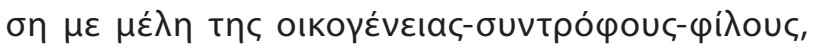




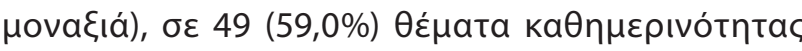

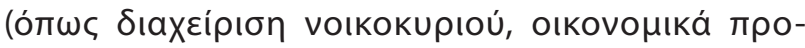

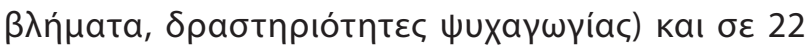

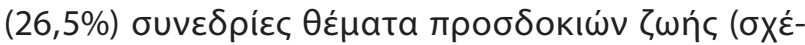

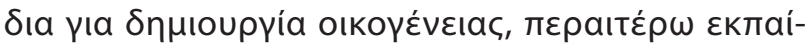

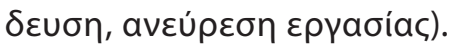

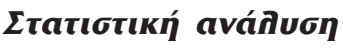

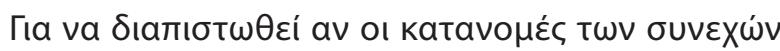

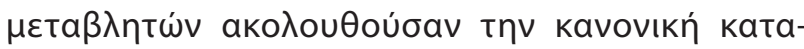

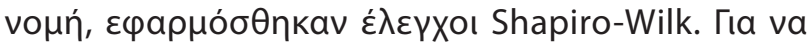

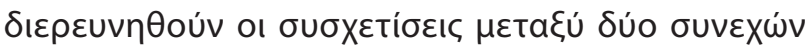

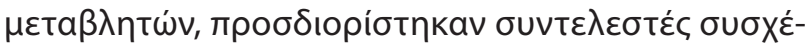

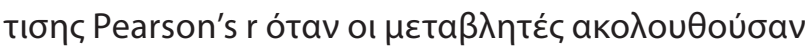

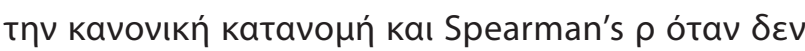

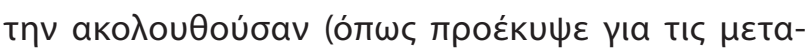

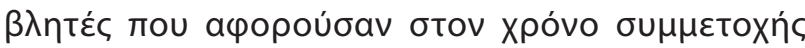

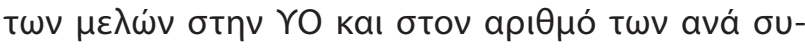

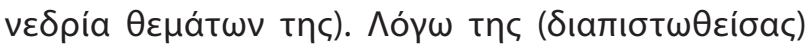

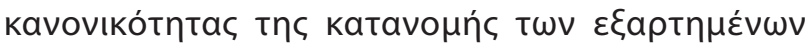

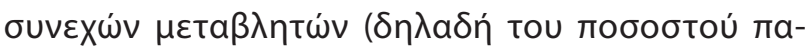

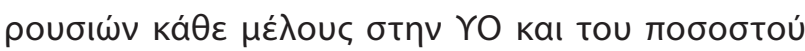

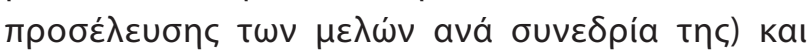

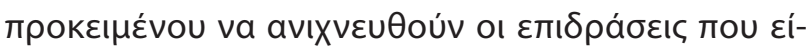

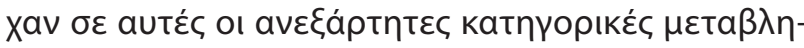

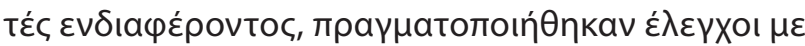

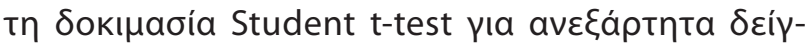

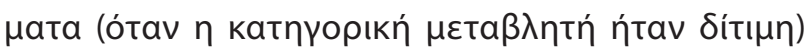

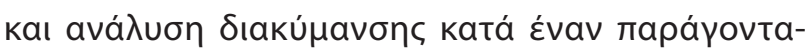
ANOVA (

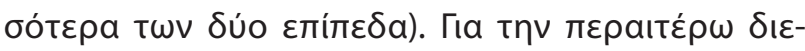

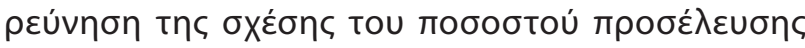

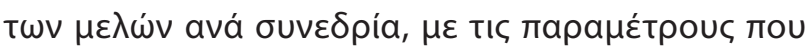

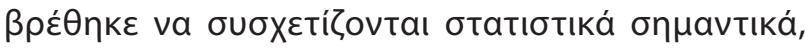

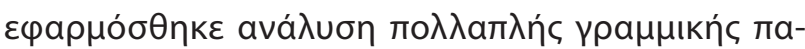

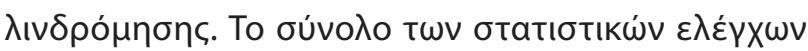

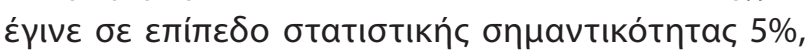

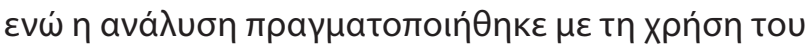

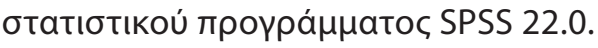

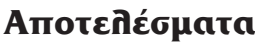

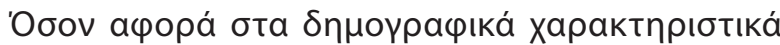

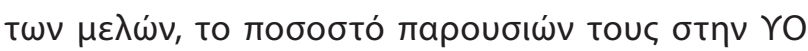

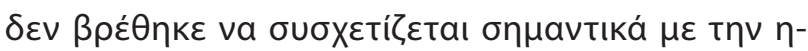

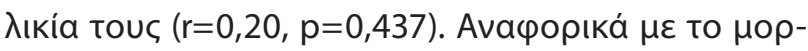

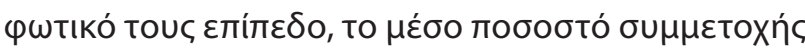

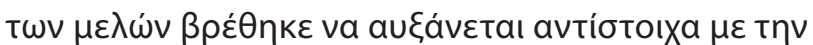

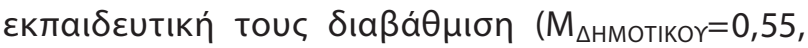

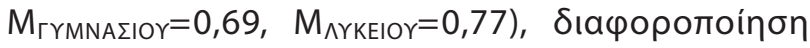

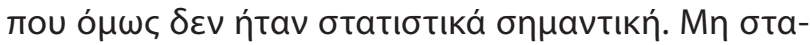

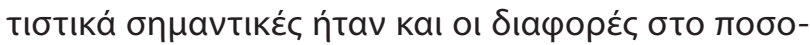

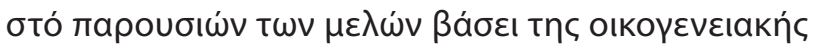

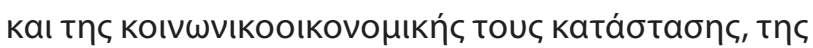

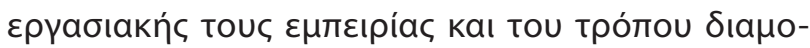

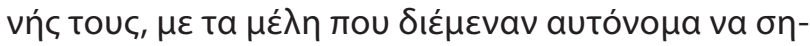

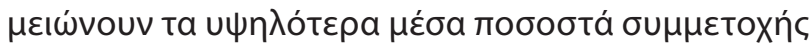

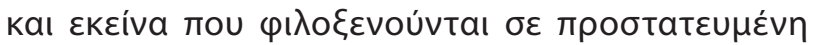

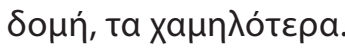

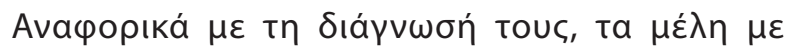

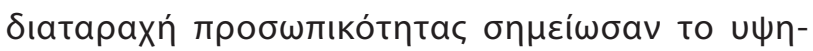

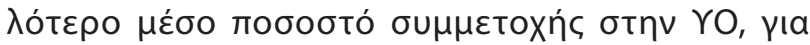

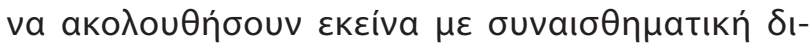

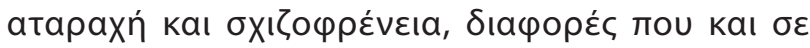

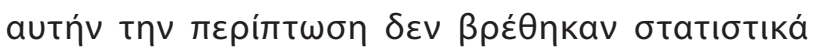

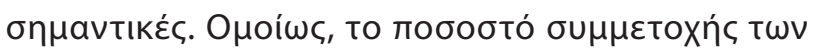

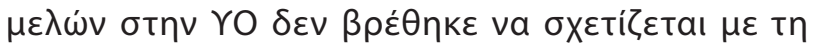

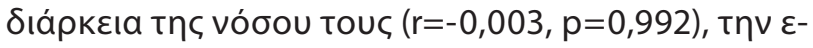

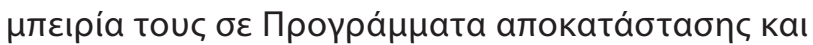

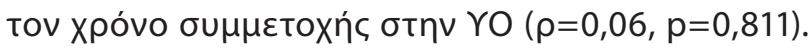

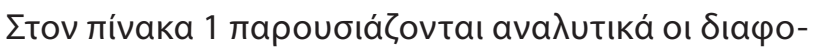

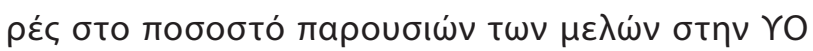

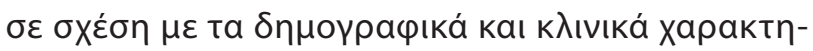
рıбтıкá тouc.

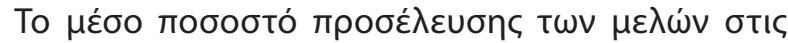

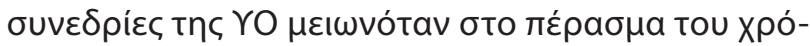
vou $\left[\left(M_{10 \text { в́ то }}=0,74\right)>\left(M_{20}\right.\right.$ в́ то $\left.=0,71\right)=\left(M_{3 \text { о в́ то }}=0,71\right)$

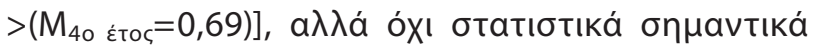

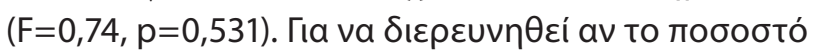

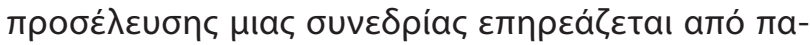

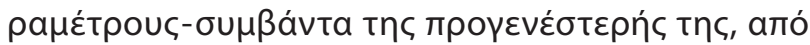

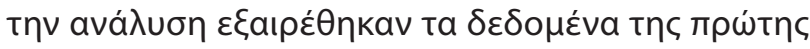

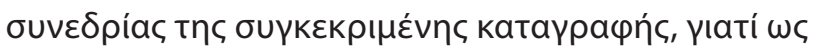

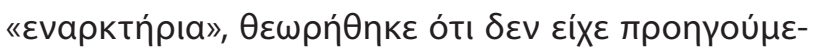

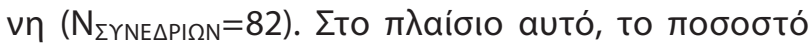

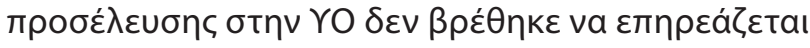

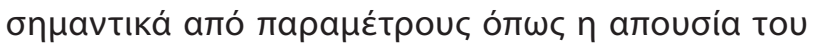

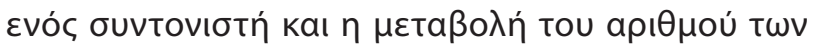

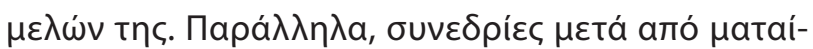

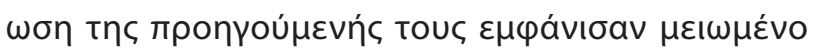

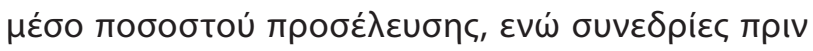

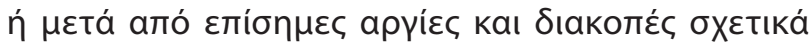




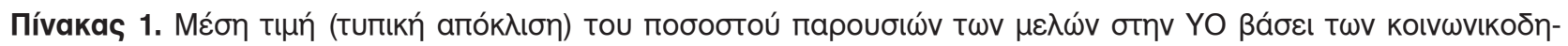

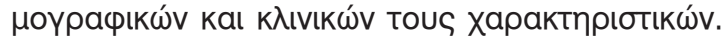

\begin{tabular}{|c|c|c|c|c|c|}
\hline Характпрібтікó & n (\%) & MO (TA) & $\mathbf{F}$ & $\mathbf{t}$ & $\mathbf{p}$ \\
\hline 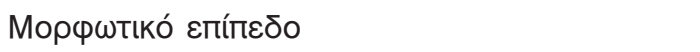 & & & 1,21 & & 0,343 \\
\hline Апо́фоітп $\triangle$ потікои́ & $4(22,2)$ & $0,55(0,31)$ & & & \\
\hline Апо́фоıтn Гuнvađíou & $2(11,1)$ & $0,69(0,15)$ & & & \\
\hline 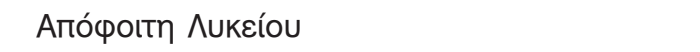 & $11(61,1)$ & $0,77(0,16)$ & & & \\
\hline 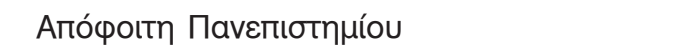 & $1(5,6)$ & & & & \\
\hline 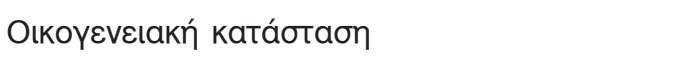 & & & 0,54 & & 0,593 \\
\hline 'Аүa & $15(83,3)$ & $0,69(0,21)$ & & & \\
\hline 'Eүүанп & $2(11,1)$ & $0,85(0,03)$ & & & \\
\hline$\Delta ı а \zeta \varepsilon \cup \gamma \mu \varepsilon ́ v \eta$ & $1(5,6)$ & & & & \\
\hline$\Delta$ ıанoví & & & 0,87 & & 0,438 \\
\hline Móvn & $3(16,7)$ & $0,83(0,14)$ & & & \\
\hline 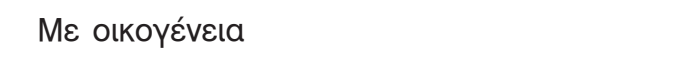 & $10(55,5)$ & $0,71(0,22)$ & & & \\
\hline 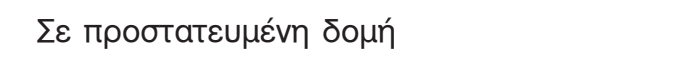 & $5(27,8)$ & $0,64(0,22)$ & & & \\
\hline 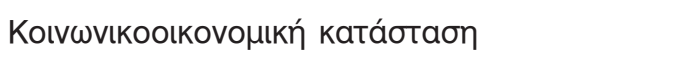 & & & 0,16 & & 0,857 \\
\hline 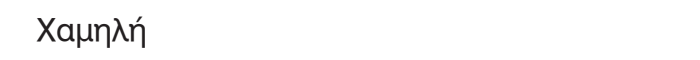 & $10(55,5)$ & $0,70(0,25)$ & & & \\
\hline Métpıa & $6(33,4)$ & $0,75(0,13)$ & & & \\
\hline 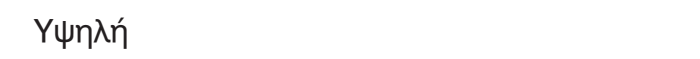 & $2(11,1)$ & $0,66(0,23)$ & & & \\
\hline 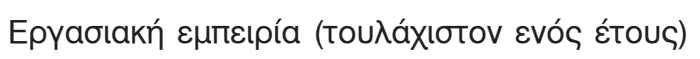 & & & & $-1,34$ & 0,216 \\
\hline Nal & $11(61,1)$ & $0,77(0,14)$ & & & \\
\hline 'OXl & $7(38,9)$ & $0,63(0,26)$ & & & \\
\hline$\Delta$ lápvwơn & & & 0,66 & & 0,533 \\
\hline 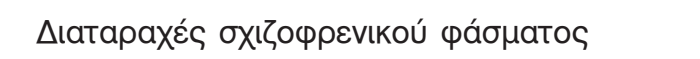 & $11(61,1)$ & $0,67(0,25)$ & & & \\
\hline 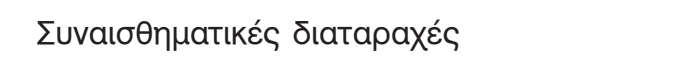 & $5(27,8)$ & $0,75(0,12)$ & & & \\
\hline 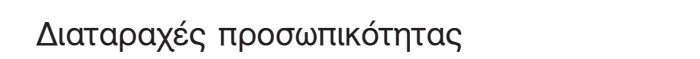 & $2(11,1)$ & $0,83(0,04)$ & & & \\
\hline 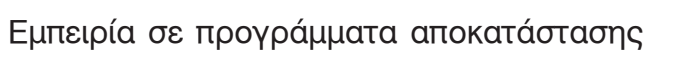 & & & & $-0,09$ & 0,993 \\
\hline Nal & $10(55,5)$ & $0,72(0,23)$ & & & \\
\hline 'OXı & $8(44,5)$ & $0,71(0,18)$ & & & \\
\hline
\end{tabular}

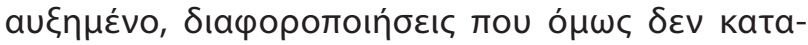

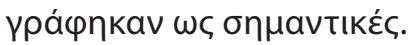

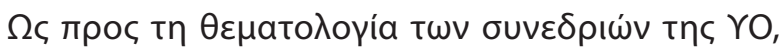

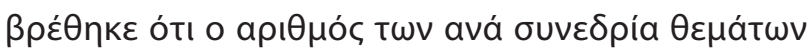

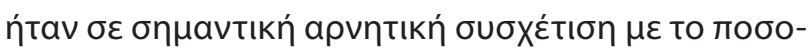

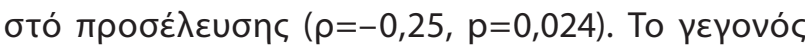

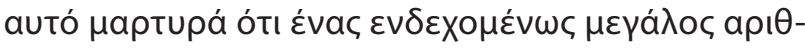

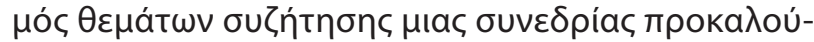

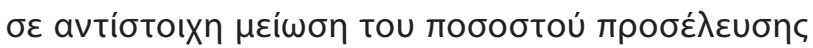

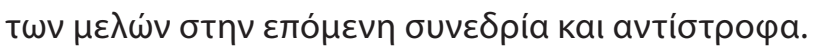

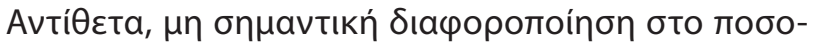

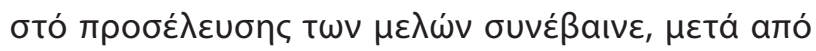

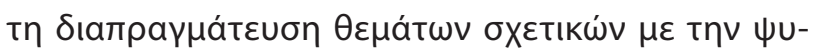

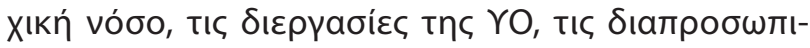




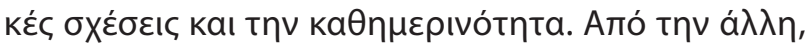

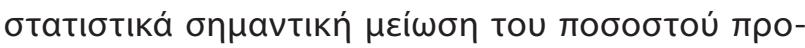

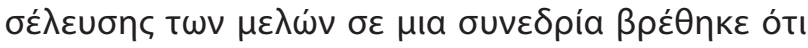

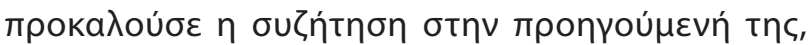

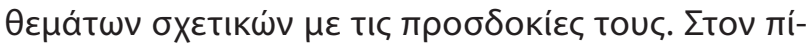

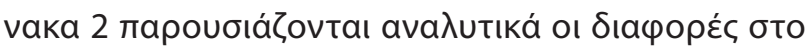

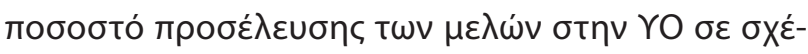

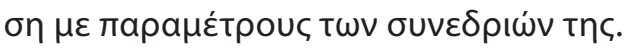

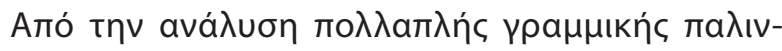

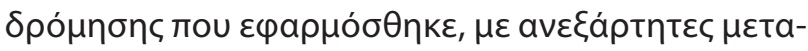

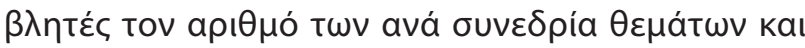

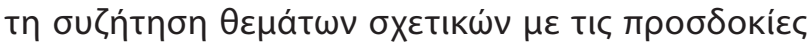

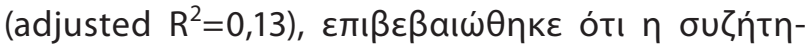

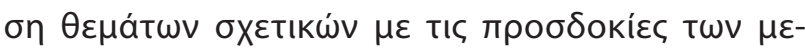

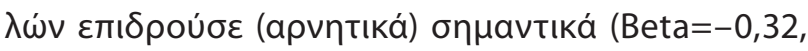

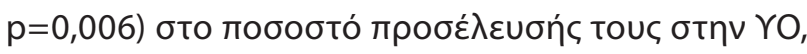

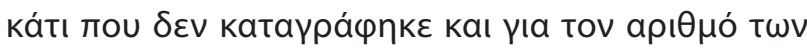

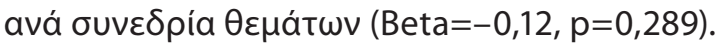

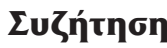

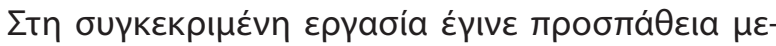

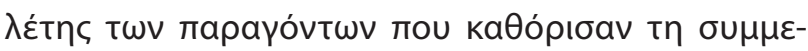

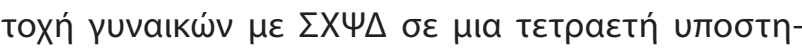

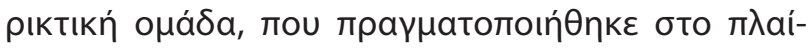

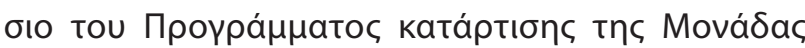

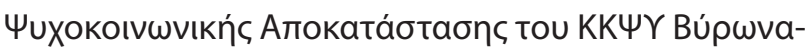

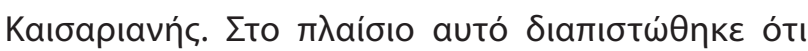

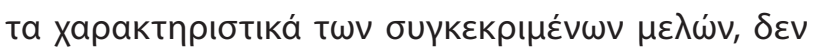

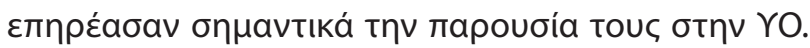

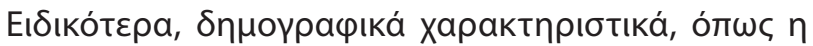

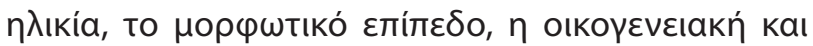

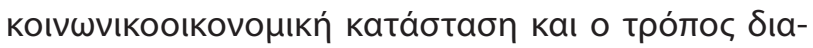

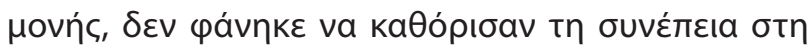

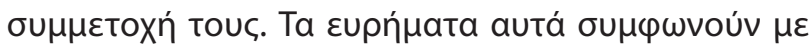

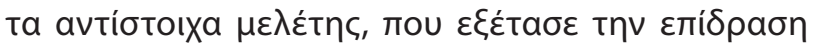

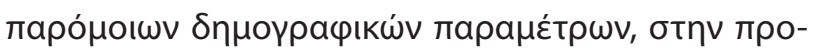

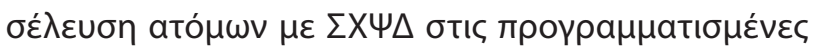

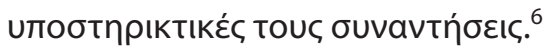

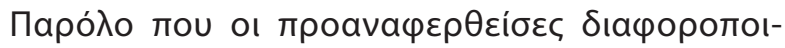

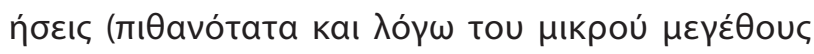

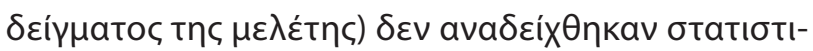

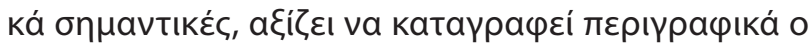

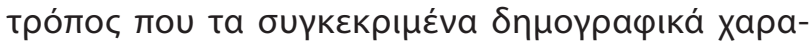

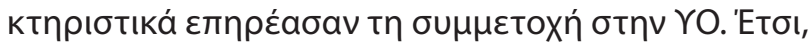

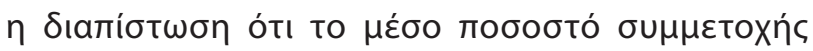

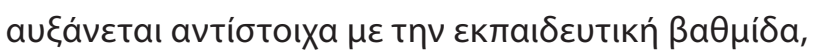

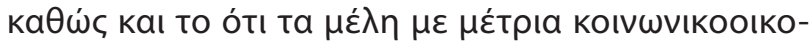

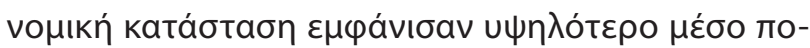

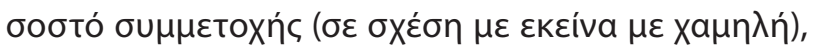

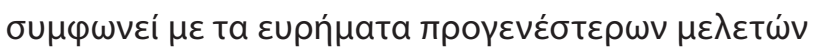

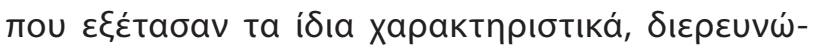

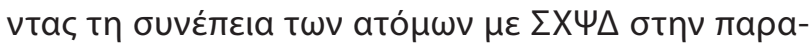

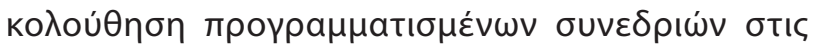

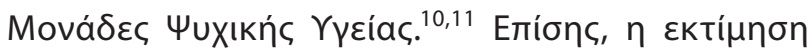

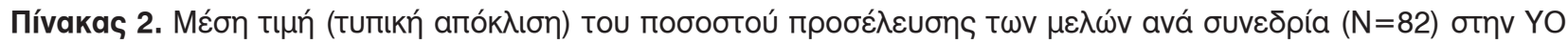

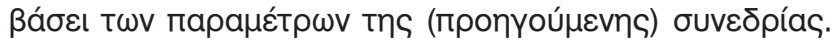

\begin{tabular}{|c|c|c|c|c|}
\hline 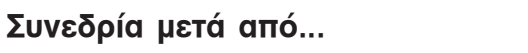 & n (\%) & MO (TA) & $\mathbf{t}$ & $\mathbf{p}$ \\
\hline 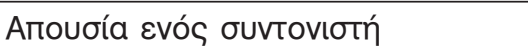 & $22(26,8)$ & $0,71(0,10)$ & $-0,19$ & 0,854 \\
\hline 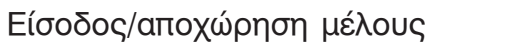 & $11(13,4)$ & $0,71(0,14)$ & $-0,03$ & 0,979 \\
\hline 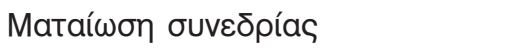 & $11(13,4)$ & $0,67(0,10)$ & 1,18 & 0,243 \\
\hline 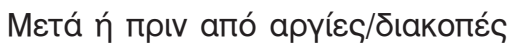 & $30(36,6)$ & $0,72(0,13)$ & $-0,68$ & 0,498 \\
\hline \multicolumn{5}{|l|}{ 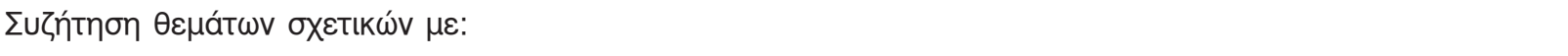 } \\
\hline 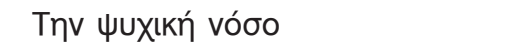 & $51(62,2)$ & $0,71(0,11)$ & 0,32 & 0,748 \\
\hline 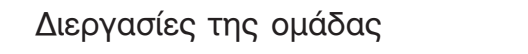 & $19(23,2)$ & $0,74(0,11)$ & $-1,45$ & 0,151 \\
\hline 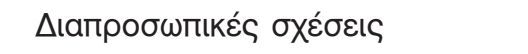 & $61(74,4)$ & $0,71(0,11)$ & 0,56 & 0,577 \\
\hline 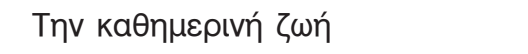 & $48(58,5)$ & $0,71(0,12)$ & 0,43 & 0,667 \\
\hline 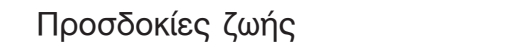 & $22(26,8)$ & $0,64(0,08)$ & 3,53 & 0,001 \\
\hline
\end{tabular}


ótı o б

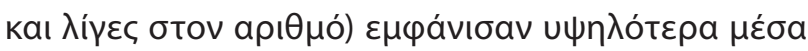

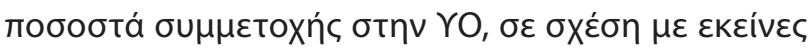

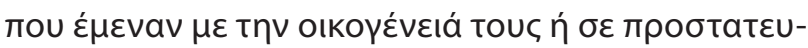

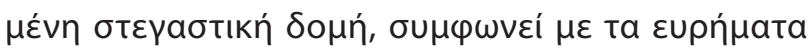

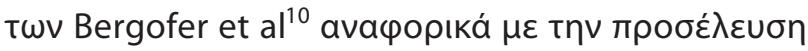

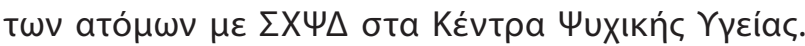

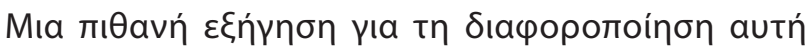

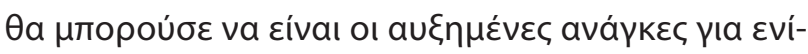

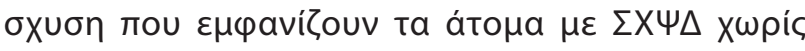

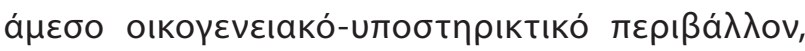

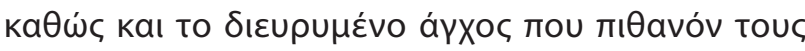

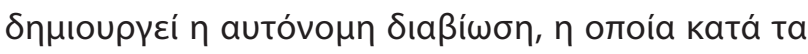

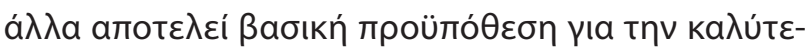

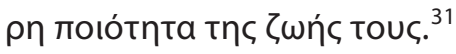

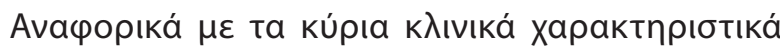

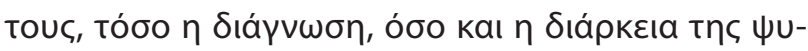

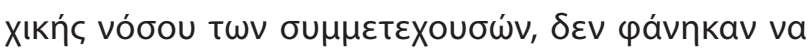

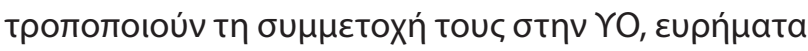

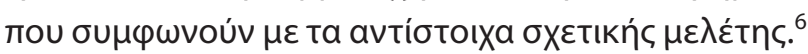

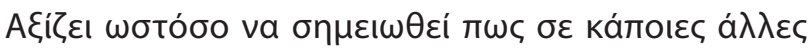

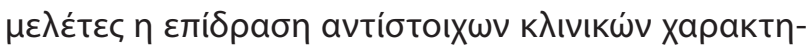

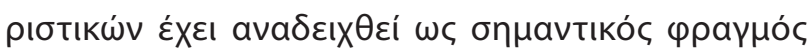

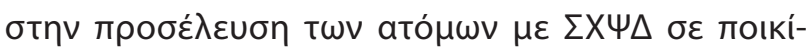

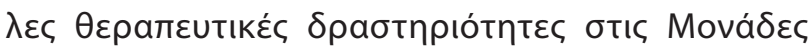

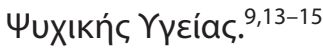

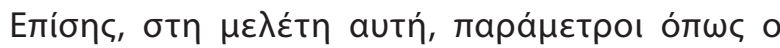

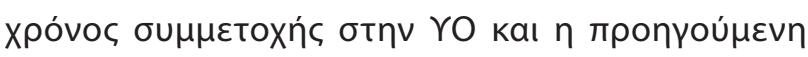

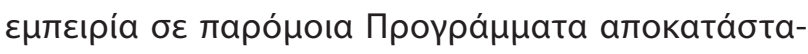

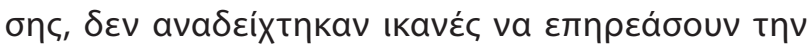

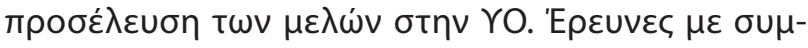

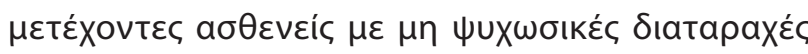

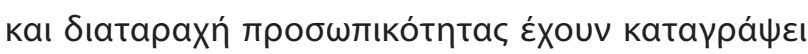

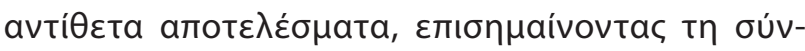

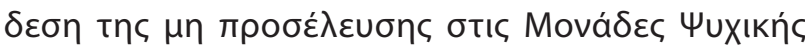

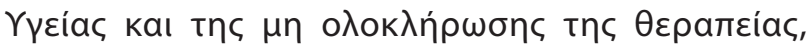

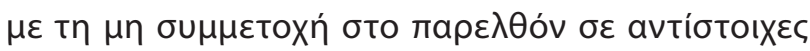

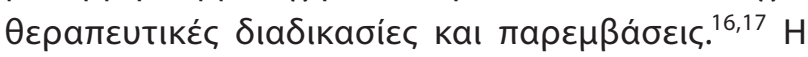

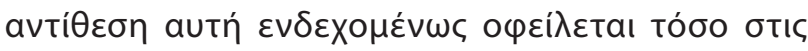

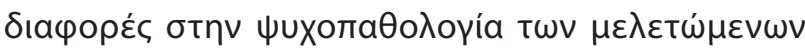

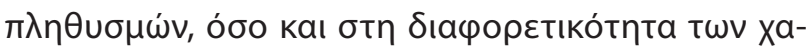

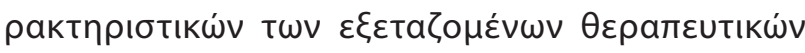

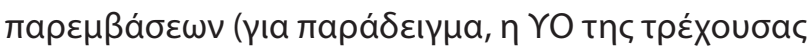

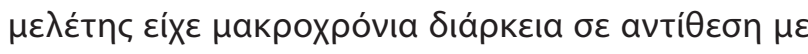

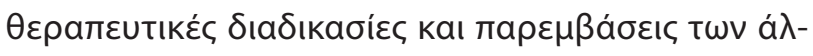
$\lambda \omega v \mu \varepsilon \lambda \varepsilon \tau \omega \dot{v})$.

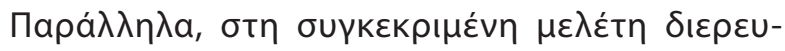

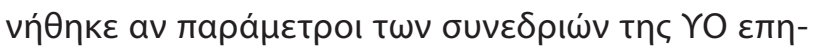

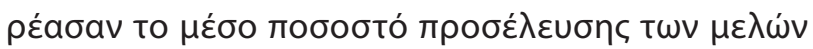

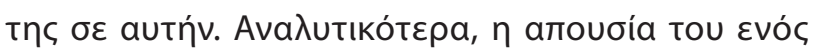

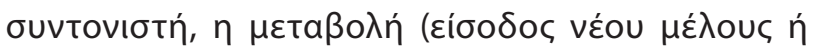

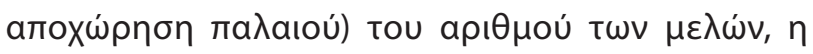

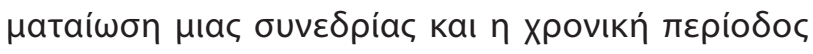

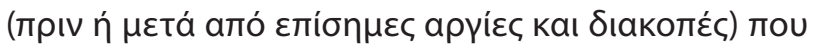

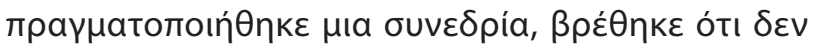

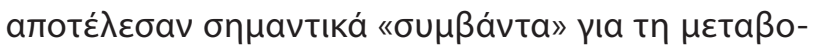

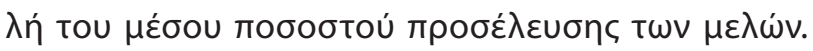

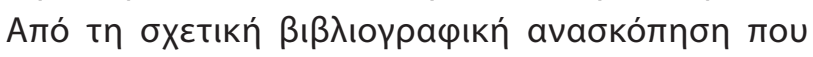

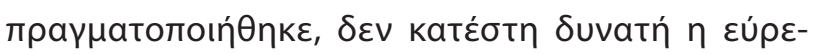

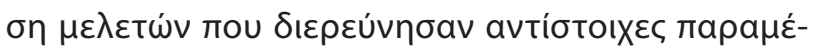

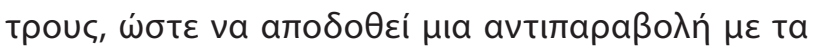

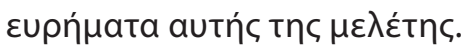

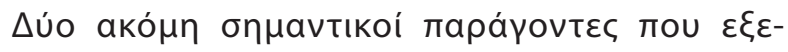

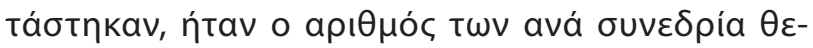

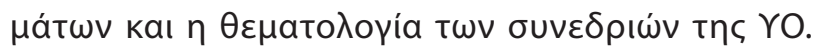

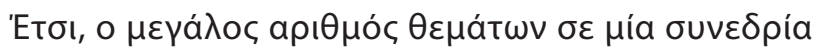

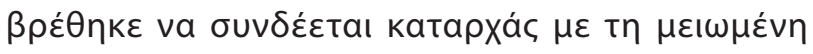

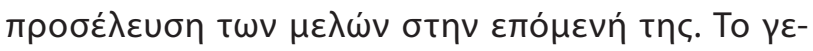

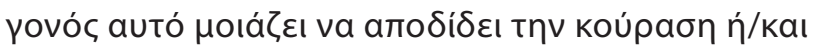

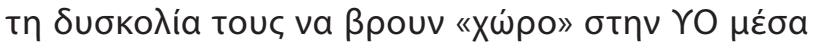

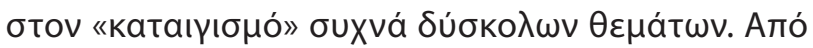

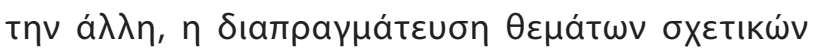

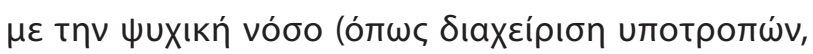

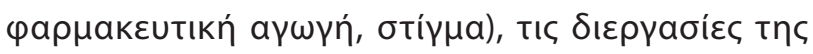

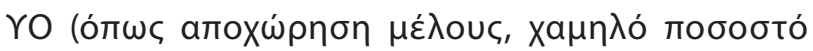

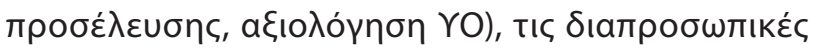

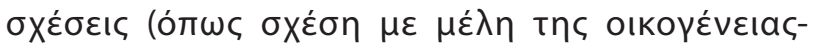

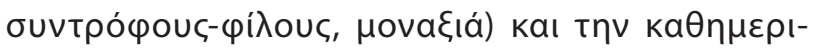

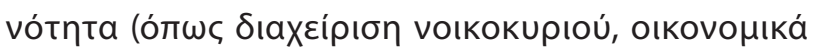

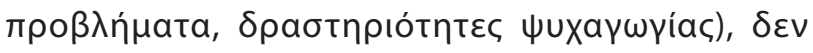

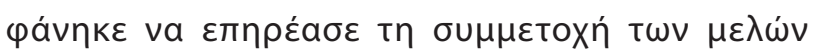

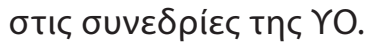

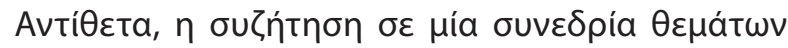

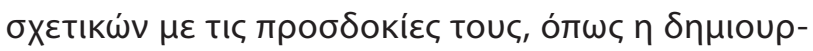

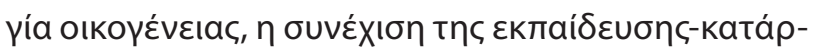

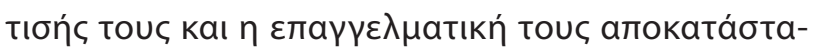

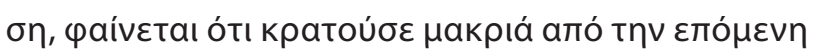

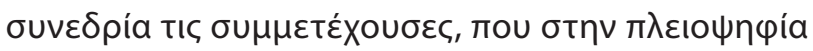

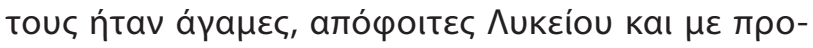




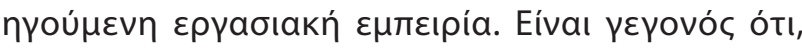

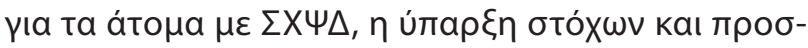

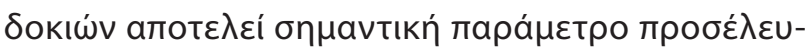

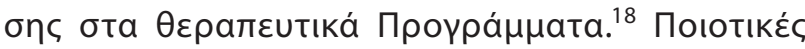

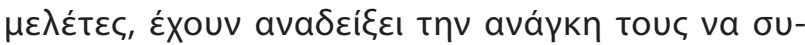

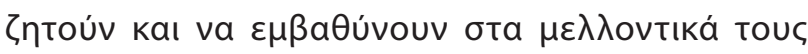

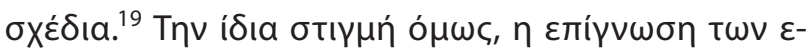

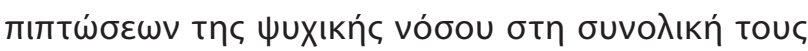

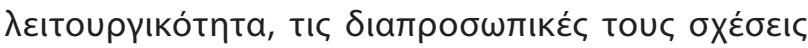

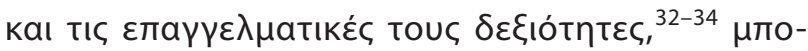

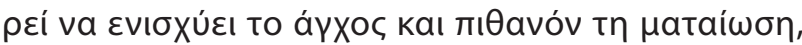

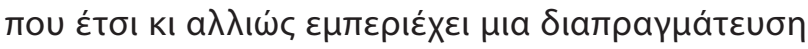

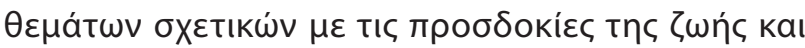

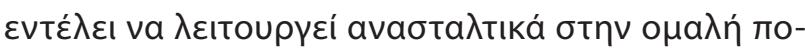

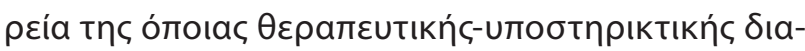

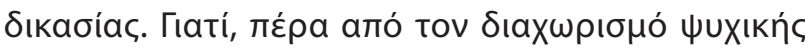

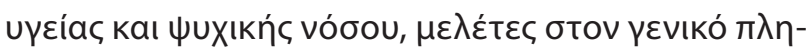

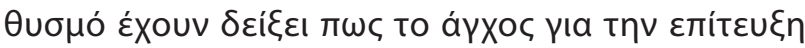

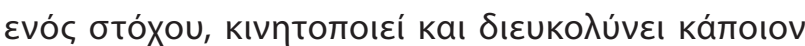

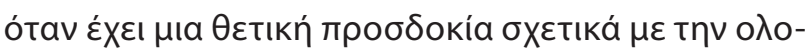

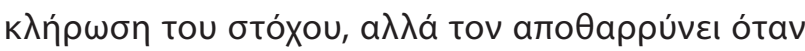

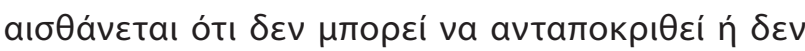

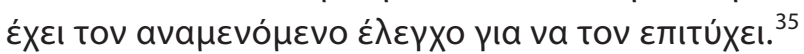

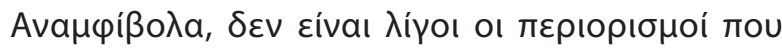

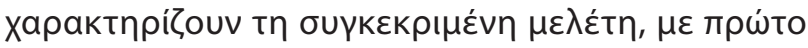

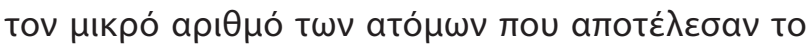

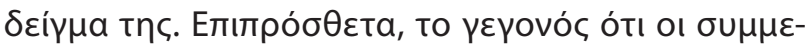

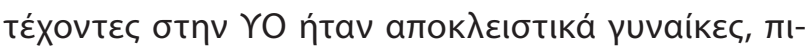

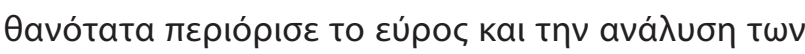

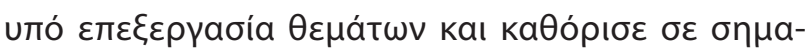

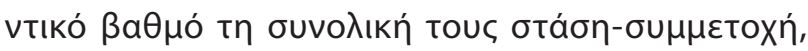

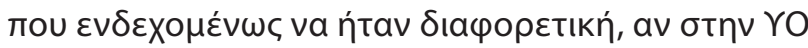

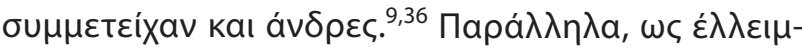

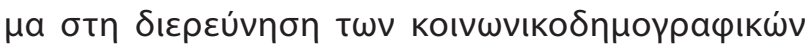

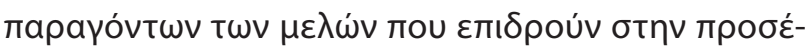

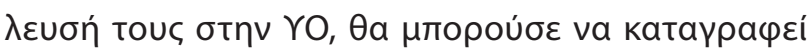

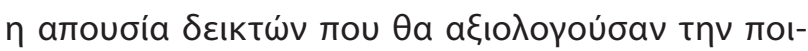

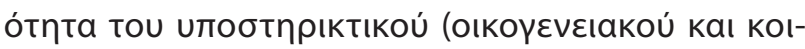

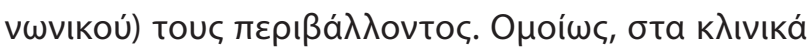

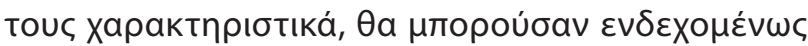

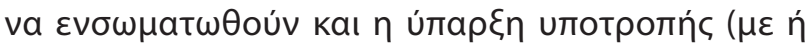

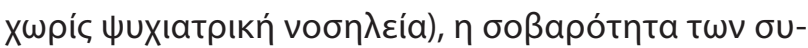

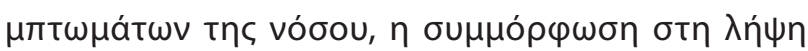

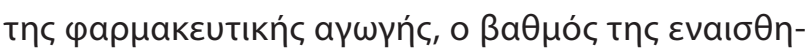

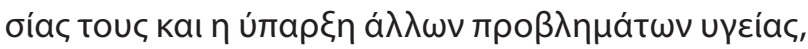

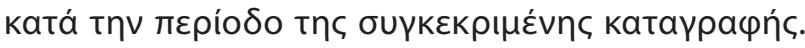

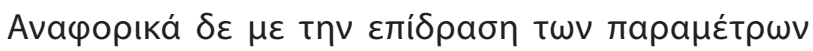

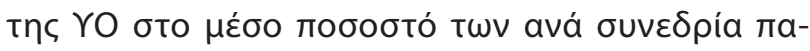

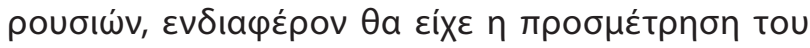

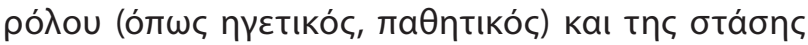

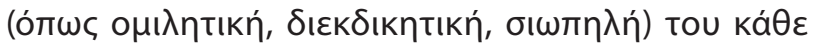

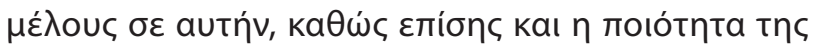

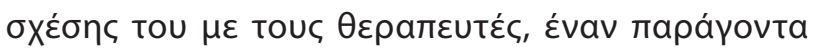

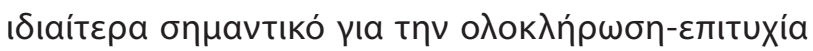

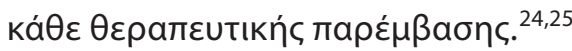

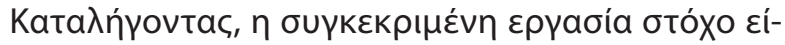

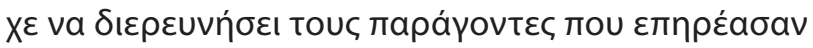

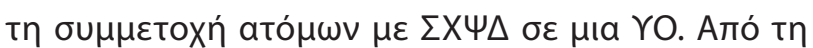

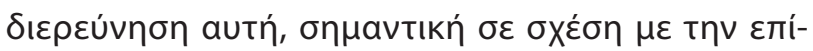

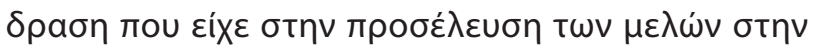

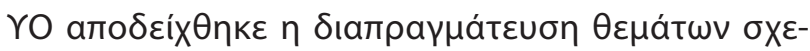

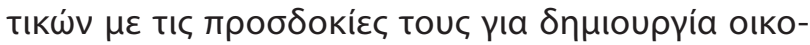

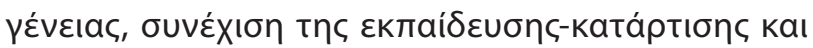

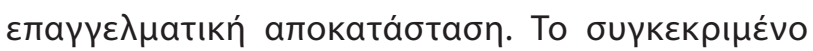

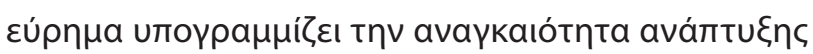

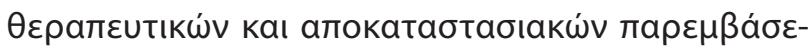

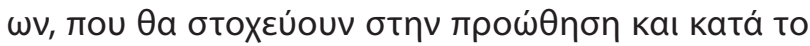

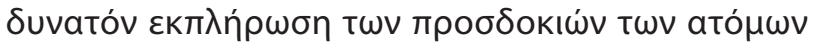

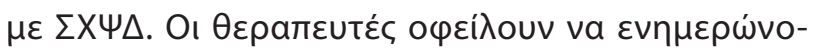

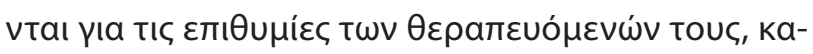

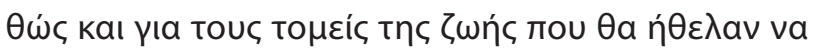

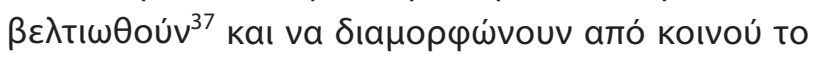

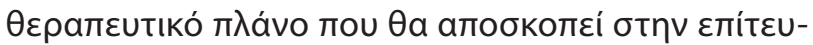

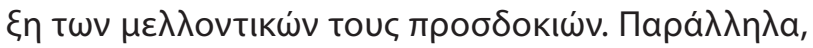

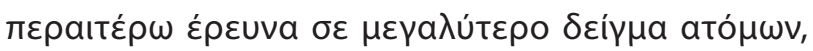

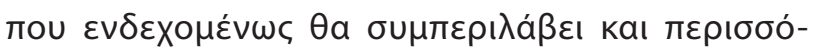

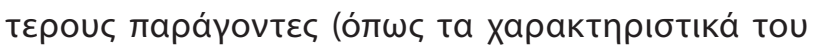

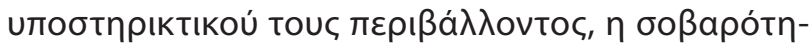

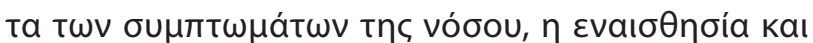

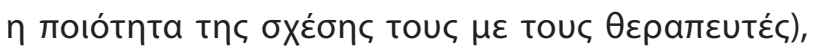

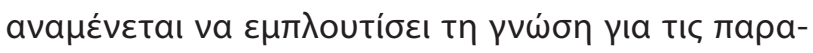

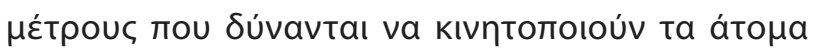

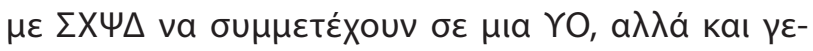

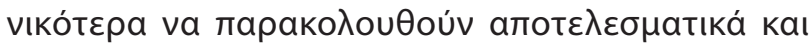

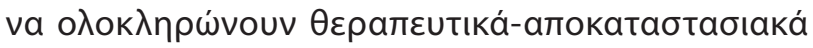

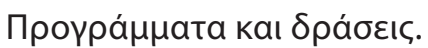

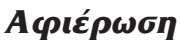

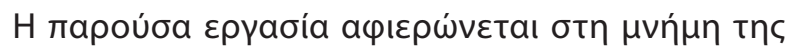

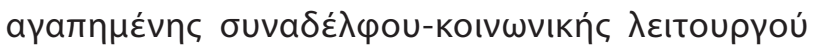

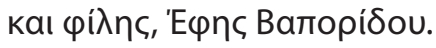




\title{
Psychosocial rehabilitation and severe mental illness: Factors that influence patients' attendance in a support group
}

\author{
Z. Kalogerakis, A. Petroutsou, A. Chatzakis, D. Ploumpidis, \\ Ch. Papageorgiou, M. Economou \\ Psychosocial Rehabilitation Unit, Byron-Kaissariani Community Mental Health Centre, \\ First Department of Psychiatry, National and Kapodistrian University of Athens, Athens, Greece
}

Psychiatriki 2019, 30:108-119

Psychosocial rehabilitation for people with chronic-severe mental illness mainly aims to social integration by restoring independent functioning in the community, improving quality of life, and addressing risk factors that lead to social disability. Support groups (SG) are usually part of this multilevel mental health process. Given that non-adherence to treatment is a common phenomenon in people with chronic- severe mental illness, the aim of the current study was to identify which factors influence members' attendance in a support group in a vocational training Program of the Psychosocial Rehabilitation Unit of Byron-Kaissariani Community Mental Health Centre. The SG sessions were weekly, with 45-minute duration, opened to any new member of the Program and coordinated by two therapists. Members' demographic and psychiatric data were gathered from the medical records of the Center. Information about SG was obtained from the reports of the sessions. The sample consisted of 18 women, with mean age $38.56( \pm 6.92)$ years. Most of them were high school graduates (61.1\%), unmarried (83.3\%), with low socioeconomic status (55.5\%), suffering from a schizophrenic spectrum disorder (61.1\%) with a mean duration $15.22( \pm 8.44)$ years. Out of 83 sessions in total, twenty-two (26.5\%) were in absence of a co-therapist, 11 (13.3\%) after a member's entrance or withdrawal and 11 (13.3\%) after a session cancellation. Furthermore, an average of four issues was discussed per session, with mental illness (62.7\%) and interpersonal relationships (73.5\%) being the most popular topics during the sessions. The statistical analysis demonstrated that members' demographic (age, education, marital status, residence, socioeconomic status, working experience) and psychiatric characteristics (diagnosis, illness duration, rehabilitation program experience) were not associated with the attendance rate in the SG. Similarly, the proportion of participants attending the sessions did not seem to be significantly related to the absence of a co-therapist, to a member's entrance or withdrawal and to a session cancellation. In contrast, attendance seemed to be significantly reduced when the topic of a session focused on members' future expectations/goals (having a family, further education, finding a job) (Beta $=-0.32$, $p=0.006)$. This finding highlights the need for future research in order to incorporate interventions that promote and address future goals and expectations of people with chronic-severe mental illness in psychosocial rehabilitation services.

Key words: Chronic-severe mental illness, support group, attendance, psychosocial rehabilitation. 


\section{BıßAtoypacpía}

1. Menear M, Briand C. Implementing a continuum of evidencebased psychosocial interventions for people with severe mental illness: part 1 - Review of major initiatives and implementation strategies. Can J Psychiatry 2014, 59:178-186, doi: $10.1177 / 070674371405900402$

2. Crawford MJ, de Jonge E, Freeman GK, Weaver T. Providing continuity of care for people with severe mental illness- A narrative review. Soc Psychiatry Psychiatr Epidemiol 2004, 39:265272, doi: 10.1007/s00127-004-0732-x

3. Castelein S, Bruggeman R, Davidson L, van der Gaag M. Creating a supportive environment: Peer support groups for psychotic disorders. Schizophr Bull 2015, 41:1211-1213, doi 10.1093/schbul/sbv113

4. Salzer MS, Shear SL. Identifying consumer-provider benefits in evaluations of consumer-delivered services. Psychiatr Rehabil J 2002, 25:281-288, doi: 10.1037/h0095014

5. Penn DL, Meyer PS, Evans E, Wirth RJ, Cai K, Burchinal M. A randomized controlled trial of group cognitive-behavioral therapy vs. enhanced supportive therapy for auditory hallucinations. Schizophr Res 2009, 109:52-59, doi: 10.1016/j. schres.2008.12.009

6. Castelein S, Bruggeman R, van Busschbach J, van der Gaag $\mathrm{M}$, Stant $\mathrm{A}$, Knegtering $\mathrm{H}$ et al. The effectiveness of peer support groups in psychosis: a randomized controlled trial. Acta Psychiatr Scand 2008, 118:64-72, doi: 10.1111/j.16000447.2008.01216.x

7. Chien WT, Thompson D. An RCT with three-year follow-up of peer persons with schizophrenia. Psychiatr Serv 2013, 64:9971005, doi: 10.1176/appi.ps.201200243

8. Davidson L, Chinman M, Sells D, Rowe M. Peer support among adults with serious mental illness: A report from the field Schizophr Bull 2006, 32:443-450, doi: 10.1093/schbul/sbj043

9. Villeneuve K, Potvin S, Lesage A, Nicole L. Meta-analysis of rates of drop-out from psychosocial treatment among persons with schizophrenia spectrum disorder. Schizophr Res 2010, 121: 266-270, doi: 10.1016/j.schres.2010.04.003

10. Berghofer G, Schmidl F, Rudas S, Steiner E, Schmitz M. Predictors of treatment discontinuity in outpatient mental health care. Soc Psychiatry Psychiatr Epidemiol 2002, 37:276-282, doi: 10.1007/s001270200020

11. Segarra R, Ojeda N, Pena J, Garcia J, Rodriguez-Morales A, Ruiz I et al. Longitudinal changes of insight in first episode psychosis and its relation to clinical symptoms, treatment adherence and global functioning: one-year follow-up from the Eiffel study. Eur Psychiatry 2012, 27:43-49, doi: 10.1016/j. eurpsy.2010.06.003

12. Tehrani E, Krussel J, Borg L, Munk-Jorgensen P. Dropping out of psychiatric treatment: A prospective study of a first-admission cohort. Acta Psychiatr Scand 1996, 94:266-271, doi: 10.1111/ j.1600-0447.1996.tb09859.x

13. Killaspy H, Banerjee S, King M, Lloyd M. Prospective controlled study of psychiatric out-patient non-attendance: Characteristics and outcome. Br J Psychiatry 2000, 176:160-165, doi: 10.1192/ bjp.176.2.160

14. Spidel A, Greaves C, Yuille J, Lecomte T. A comparison of treatment adherence in individuals with a first episode of psychosis and inpatients with psychosis. Int J Law Psychiatry 2015, 39:90-98, doi: 10.1016/j.ijlp.2015.01.026

15. Tsang HW, Fung KM, Chung RC. Self-stigma and stages of change as predictors of treatment adherence of individuals with schizophrenia. Psychiatry Res 2010, 180:10-15, doi: 10.1016/j. psychres.2009.09.001

16. Fenger M, Mortensen E, Poulsen S, Lau M. No-shows, drop-outs and completers in psychotherapeutic treatment: Demographic and clinical predictors in a large sample of nonpsychotic patients. Nord J Psychiatry 2011, 65:183-191, doi: 10.3109/08039488.2010.515687

17. Ogrodniczuk JS, Joyce AS, Lynd LD, Piper WE, Steinberg $\mathrm{PI}$, Richardson K. Predictors of premature termination of day treatment for personality disorder. Psychother Psychosom 2008, 77:365-371, doi: 10.1159/000151390

18. Corriss DJ, Smith TE, Hull JW, Lim RW, Pratt SI, Romanelli S. Interactive risk factors for treatment adherence in a chronic psychotic disorders population. Psychiatry Res 1999, 89:269-274, doi: 10.1016/S0165-1781(99)00111-0

19. Gunnmo P, Fatouros-Bergman H. What do individuals with schizophrenia need to increase their well-being. Int $J$ Qual Stud Heal Well-being 2011, 6:1-11, doi: 10.3402/qhw.v6i1.5412

20. Fung KMT, Tsang HWH, Corrigan PW. Self-stigma of people with schizophrenia as predictor of their adherence to psychosocial treatment. Psychiatr Rehabil J 2008, 32:485-495, doi: 10.2975/32.2.2008.95.104

21. Bryson G, Lysaker P, Bell M. Quality of life benefits of paid work activity in schizophrenia. Schizophr Bull 2002, 28:249-257, PMID: 12693431

22. Rossi A, Amaddeo F, Bisoffi G, Ruggeri M, Thornicroft G, Tansella M. Dropping out of care: Inappropriate terminations of contact with community-based psychiatric services. $\mathrm{Br} \mathrm{J}$ Psychiatry 2002, 181:331-338, doi: 10.1192/bjp.181.4.331

23. Thompson L, McCabe R. The effect of clinician-patient alliance and communication on treatment adherence in mental health care: A systematic review. BMC Psychiatry 2012, 12:87, doi: 10.1186/1471-244X-12-87

24. Bentall R, Tarrier N, Lewis S, Haddock G, Kinderman P, Kingdon D et al. The therapeutic alliance in early psychosis. Acta Psychiatr Scand 2002, 106:69-106, doi: 10.1034/j.1600-0447.106.s413.1_ 119.x

25. Lecomte T, Spidel A, Leclerc C, MacEwan GW, Greaves C, Bentall RP. Predictors and profiles of treatment nonadherence and engagement in services problems in early psychosis. Schizophr Res 2008, 102:295-302, doi: 10.1016/j. schres.2008.01.024 
26. Barry MJ, Edgman-Levitan S. Shared decision making - The pinnacle of patient centered care. N Engl J Med 2012, 366:780-781, doi: 10.1056/NEJMp1109283

27. Kane J, Kishimoto T, Correll C. Non-adherence to medication in patients with psychotic disorders: Epidemiology, contributing factors and management strategies. World Psychiatry 2013, 12:216-226, doi: 10.1002/wps.20060

28. Slade $M$. Implementing shared decision making in routine mental health care. World Psychiatry 2017, 16:146-153, doi: 10.1002/wps.20412

29. Kreyenbuhl J, Nossel IR, Dixon LB. Disengagement from mental health treatment among individuals with schizophrenia and strategies for facilitating connections to care: A review of the literature. Schizophr Bull 2009, 35:696-703, doi: 10.1093/schbul/sbp046

30. Nosé M, Barbui C, Tansella M. How often do patients with psychosis fail to adhere to treatment programmes? A systematic review. Psychol Med 2003, 33:1149-1160, doi: 10.1017/ S0033291703008328

31. Hansson L, Middelboe T, Merinder L, Bjarnason O, BengtssonTops A, Nilsson $L$ et al. Predictors of subjective quality of life in schizophrenic patients living in the community. A Nordic multicentre study. Int J Soc Psychiatry 1999, 45:247-258, doi: 10.1177/002076409904500403

32. Evensen S, Wisløff T, Lystad JU, Bull H, Ueland T, Falkum E. Prevalence, employment rate, and cost of schizophrenia in a high-income welfare society: A population-based study using comprehensive health and welfare registers. Schizophr Bull 2015, 42:476-483, doi: 10.1093/schbul/sbv141
33. Winokur G, Tsuang M. The natural history of mania, depression, and schizophrenia. American Psychiatric Press, Washington, 1996

34. Jobe T, Harrow M. Long-term outcome of patients with schizophrenia: A review. Can J Psychiatry 2005, 50:892-900, doi: 10.1177/070674370505001403

35. Jones G, Hanton S. Interpretation of competitive anxiety symptoms and goal attainment expectancies. J Sport Exerc Psychol 1996, 18:144-157, doi: 10.1123/jsep.18.2.14

36. Pure DL. Single-gender or mixed-gender groups: Choosing a perspective. In: Kleinberg JL (ed) The Wiley-Blackwell Handbook of Group Psychotherapy. Wiley-Blackwell, Chichester, 2012

37. Priebe S, McCabe R, Bullenkamp J, Hansson L, Lauber C, Martinez-Leal $\mathrm{R}$ et al. Structured patient-clinician communication and 1-year outcome in community mental healthcare: cluster randomised controlled trial. Br J Psychiatry 2007, 191:420426, doi: 10.1192/bjp.bp.107.036939

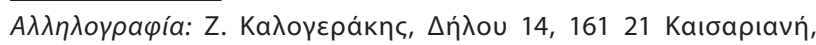
$\operatorname{T\eta \lambda }$ : 210-76 44 705, 210-76 61 069, e-mail: zachkalo@med.uoa.gr 


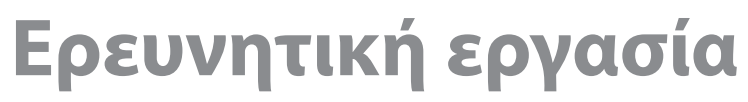 Research article
}

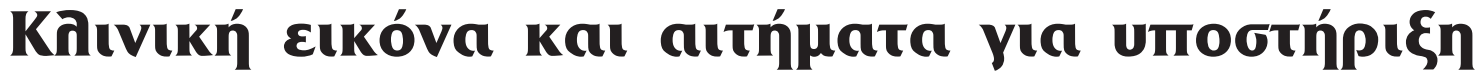

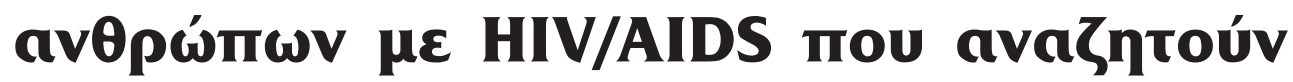

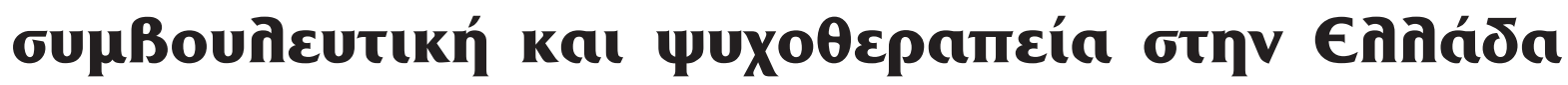

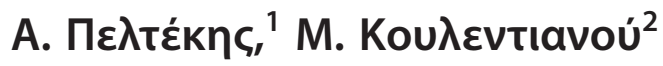

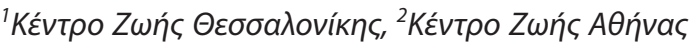

廿uxıатрıкń 2019, 30:120-128

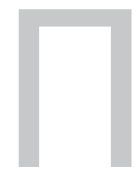

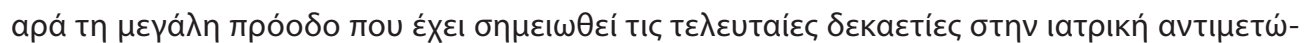

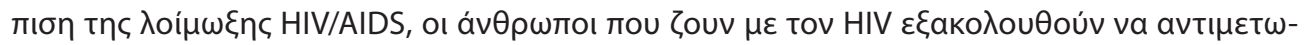

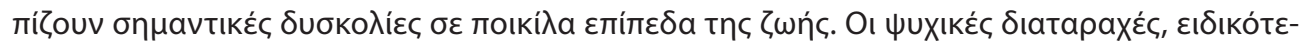

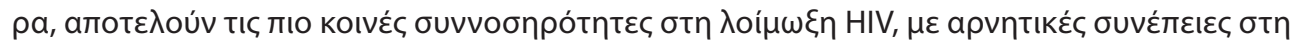

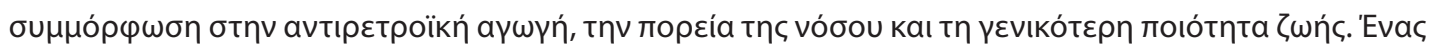

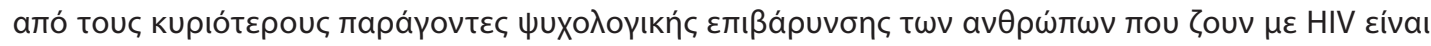

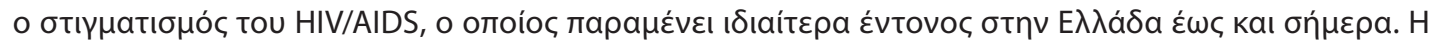

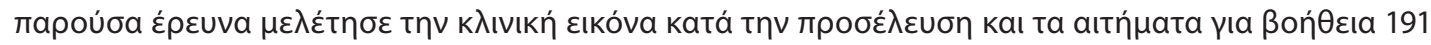

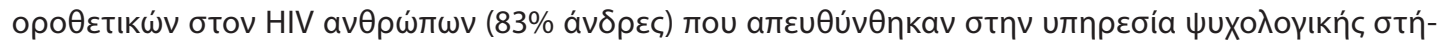

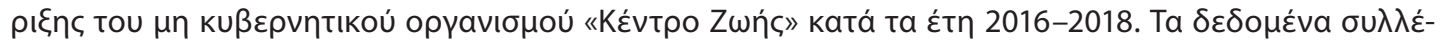

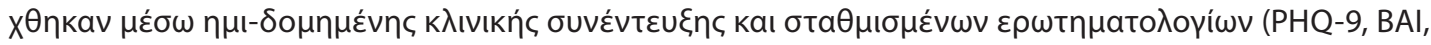

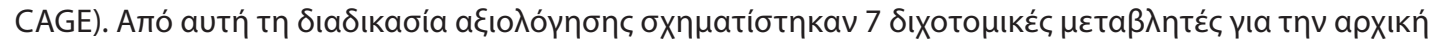

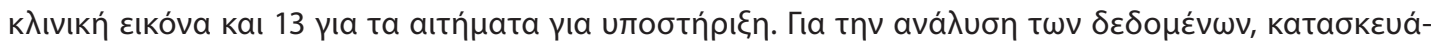

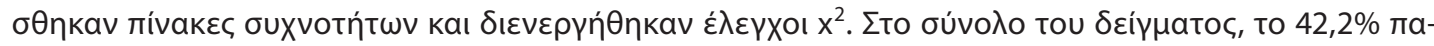

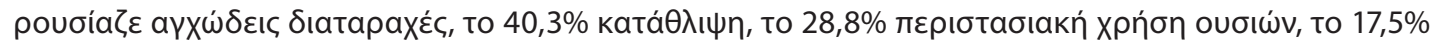

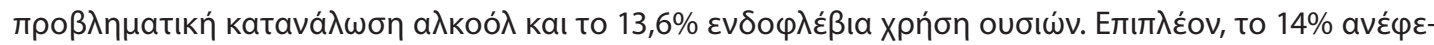

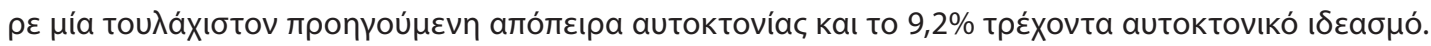

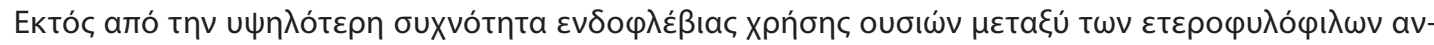

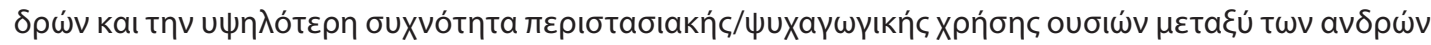

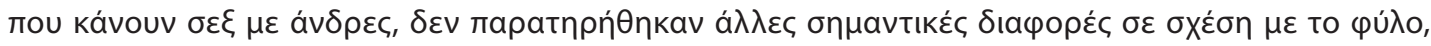

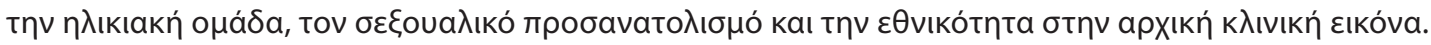

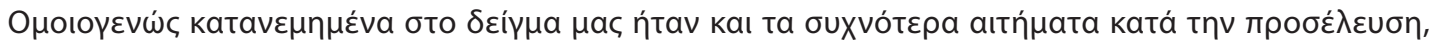




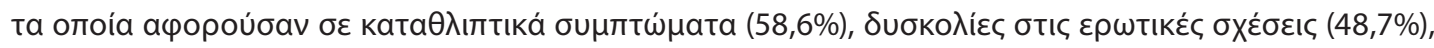

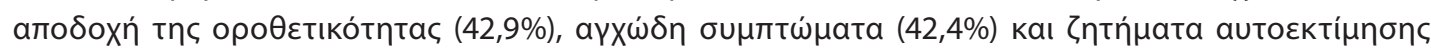

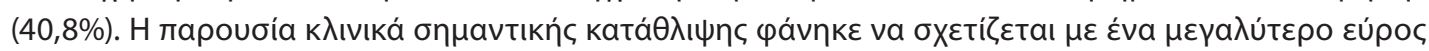

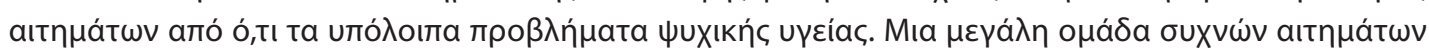

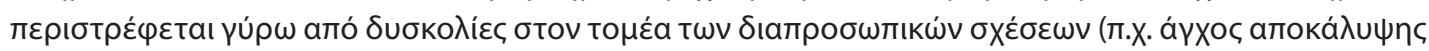

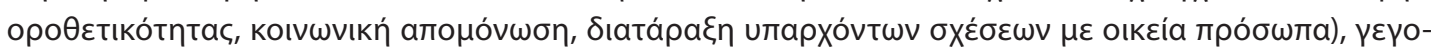

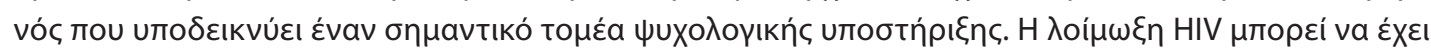

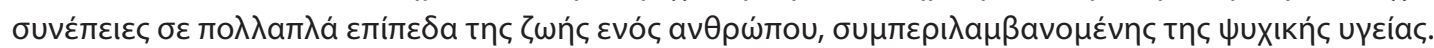

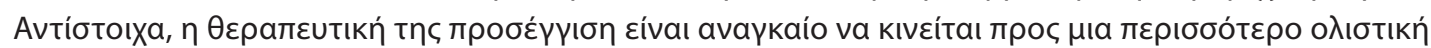

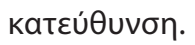

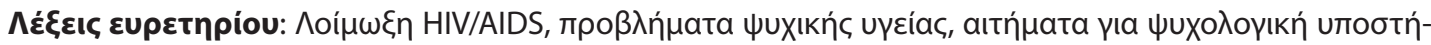

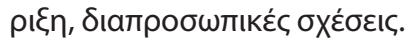

\section{Eıбaywyń}

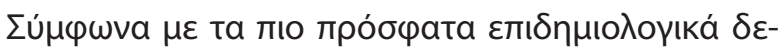

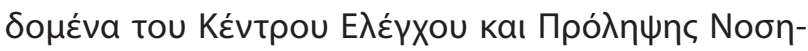

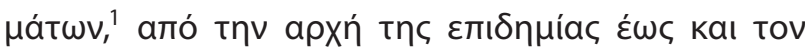

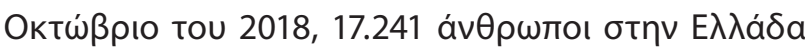

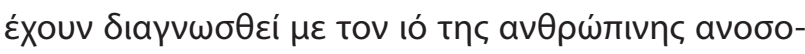

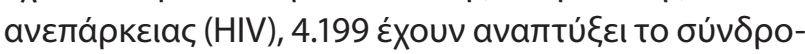

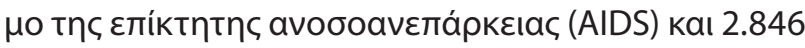

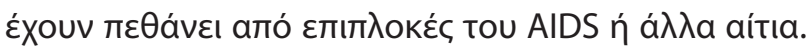

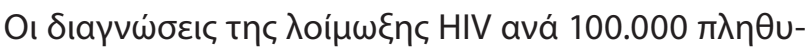

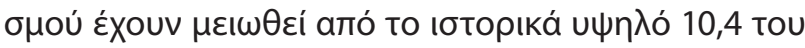

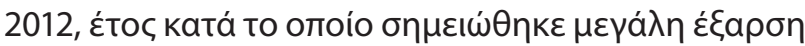

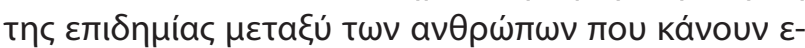

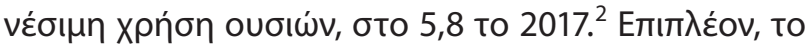

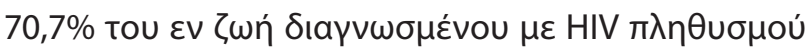

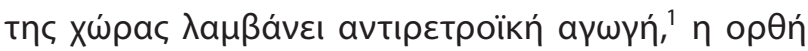

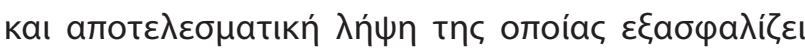

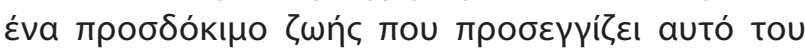

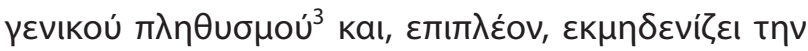

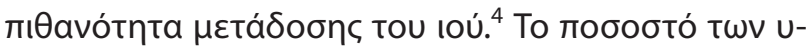

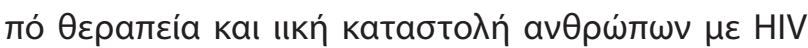

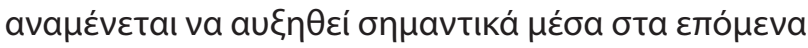

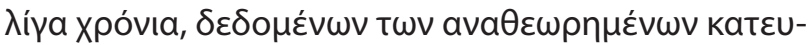

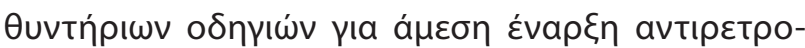

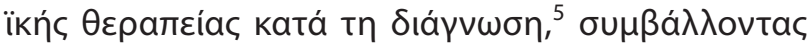

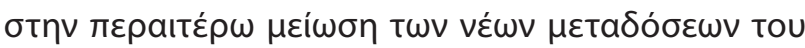

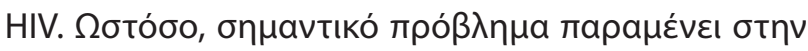

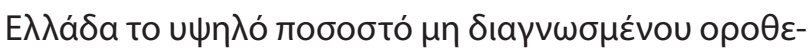

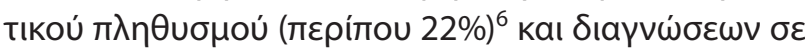

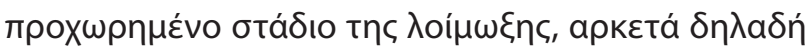

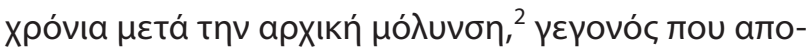

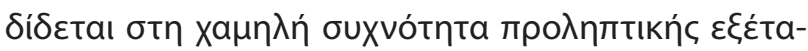

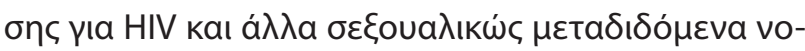

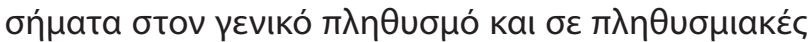

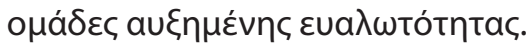

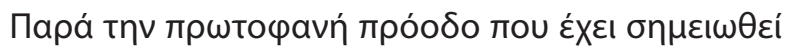

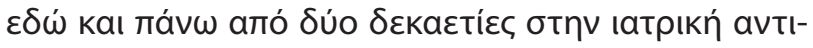

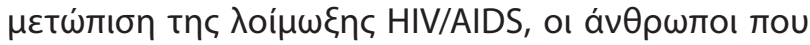

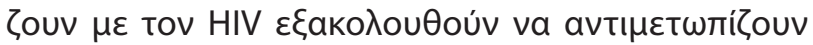

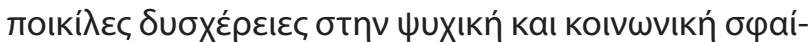

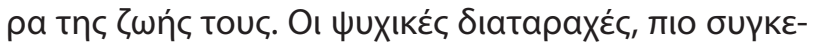

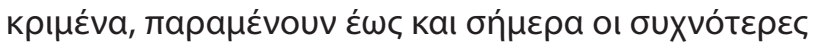

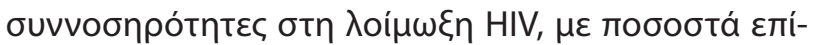

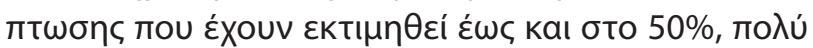

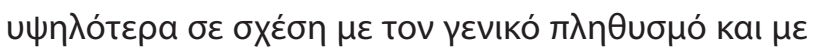

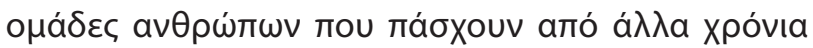

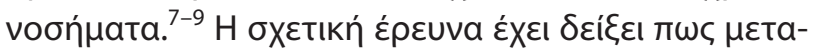

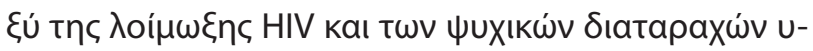

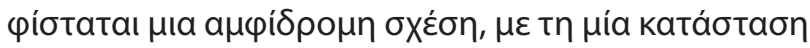

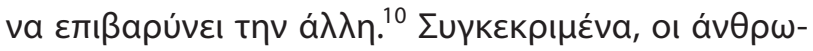

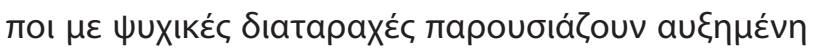

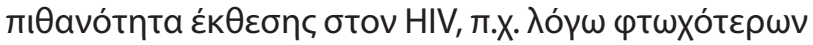

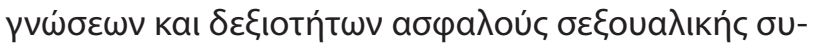

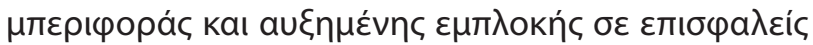

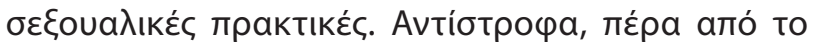

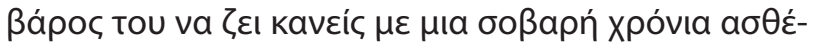

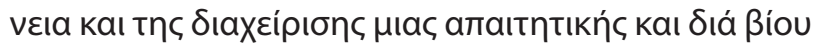

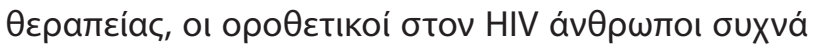

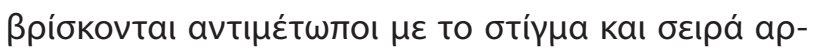

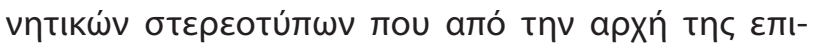

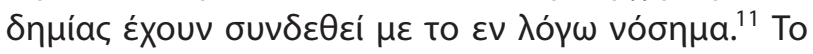

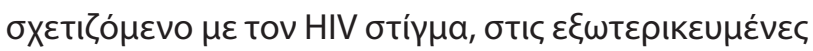

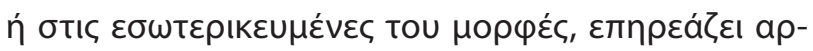

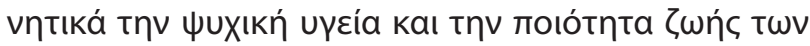

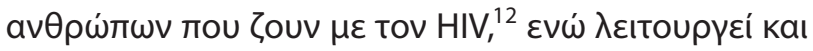

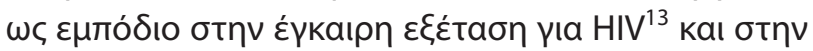

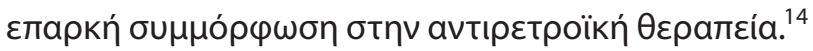




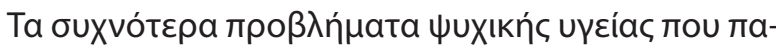

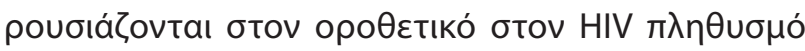

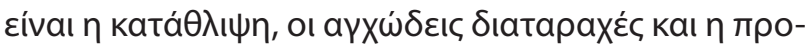

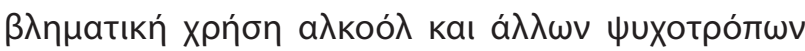

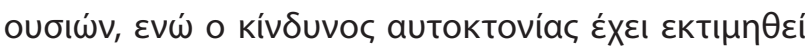

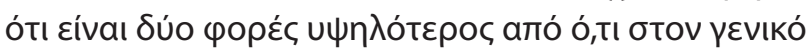

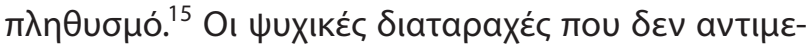

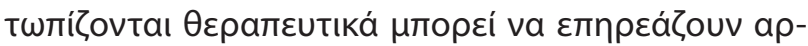

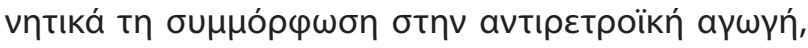

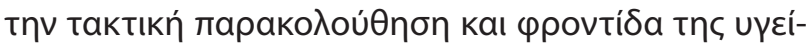

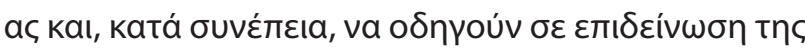

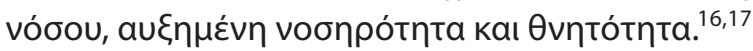

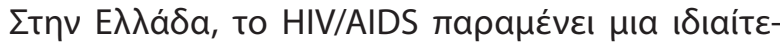

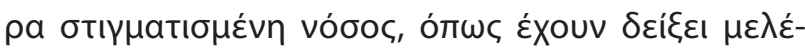

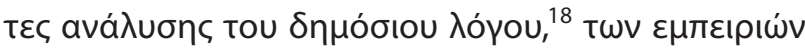

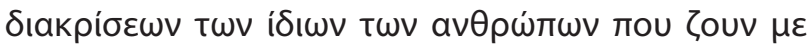

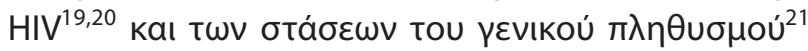

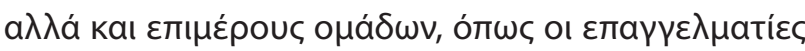

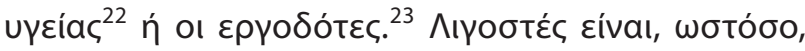

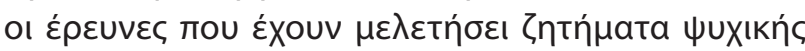

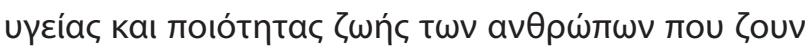

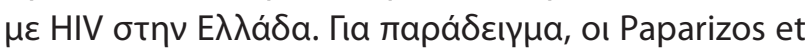

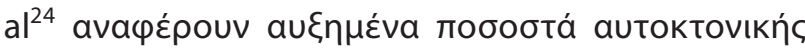

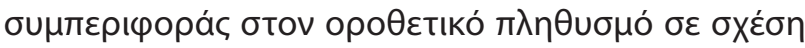

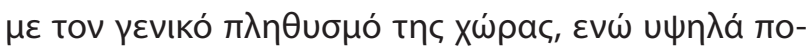

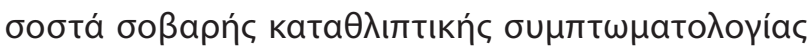

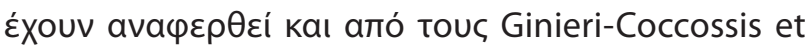

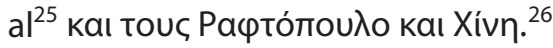

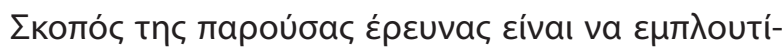

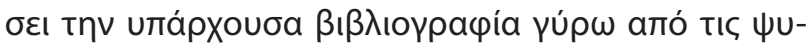

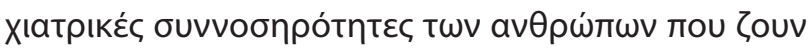

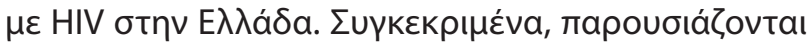

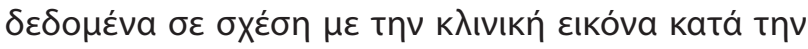

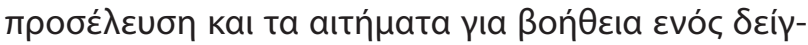

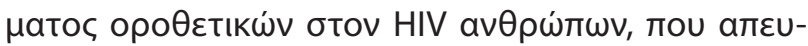

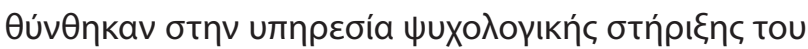

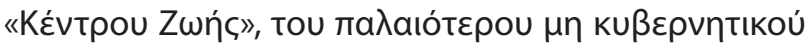

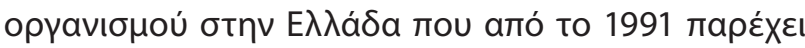

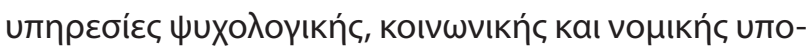

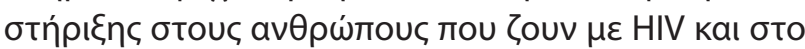

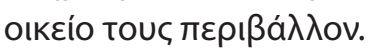

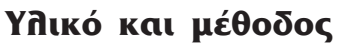

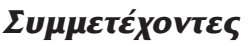

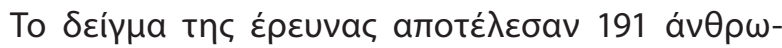

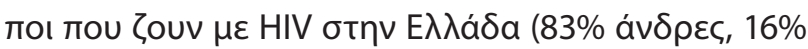

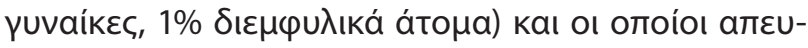

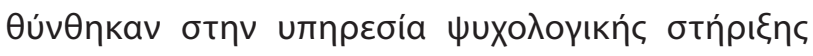

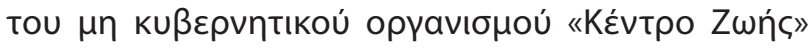

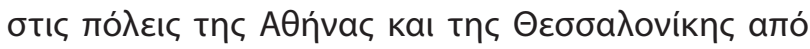

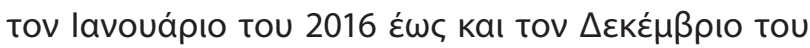

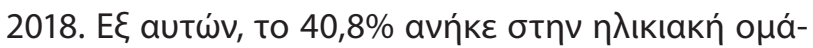

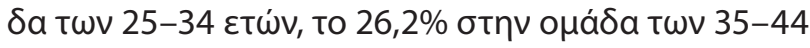

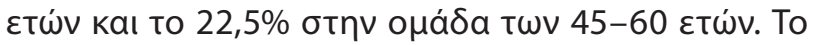

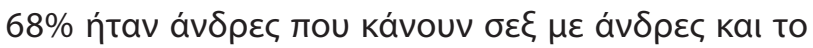
13,6\% ávӨ

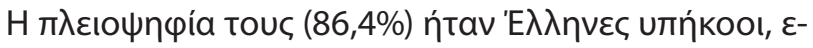

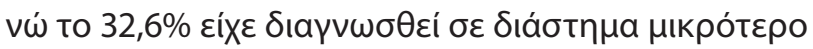

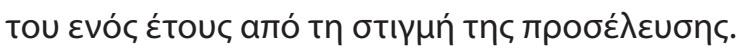

\section{Мıаঠıкабі́a}

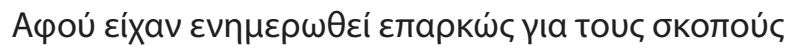

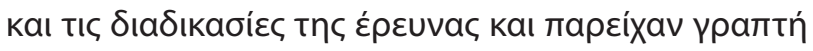

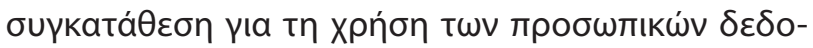

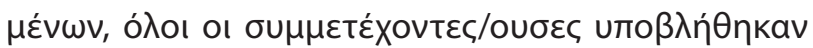

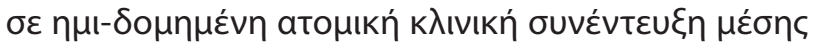

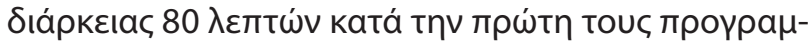

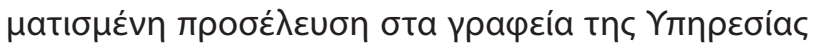

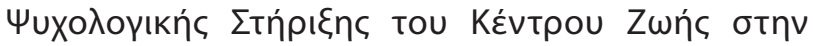

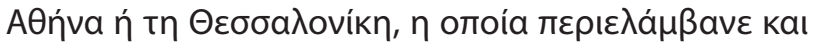

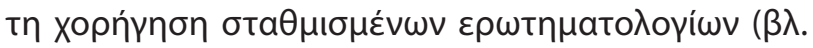

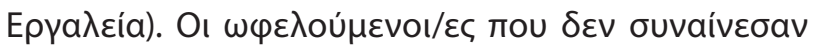

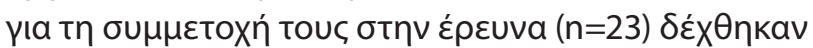

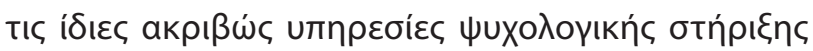

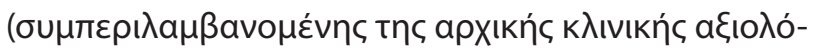

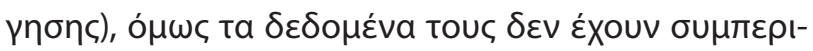

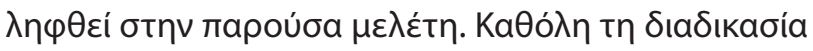

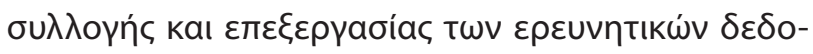

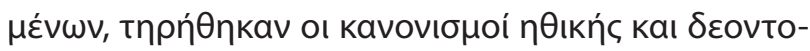

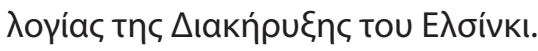

\section{Epyaneía}

Oı ато

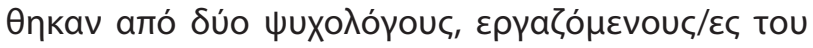

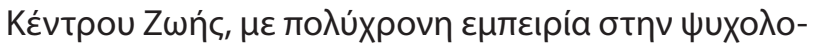

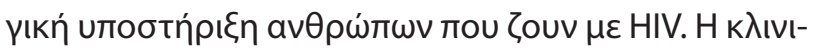

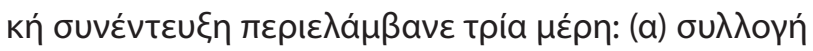

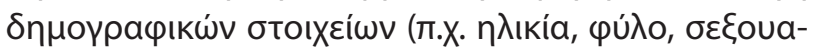

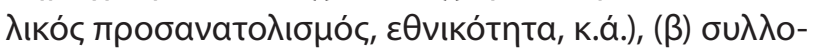

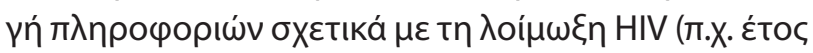

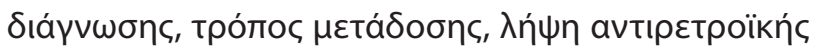

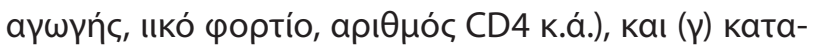

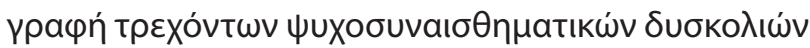

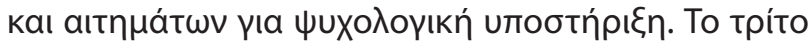

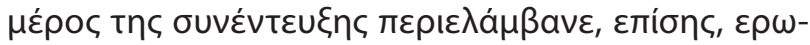




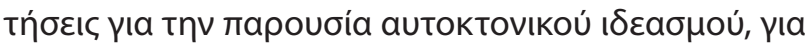

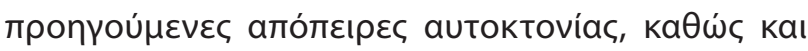

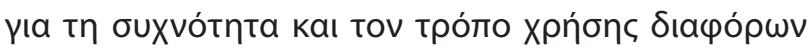

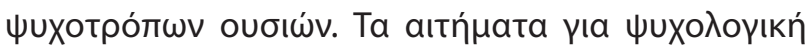

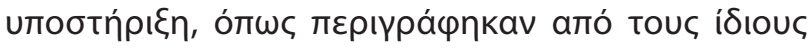

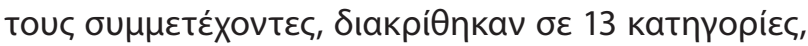

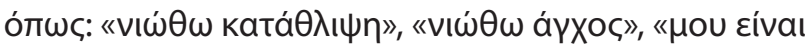

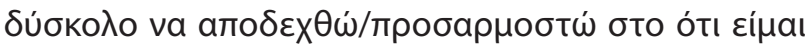

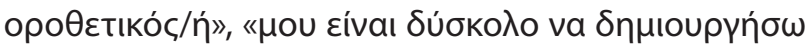

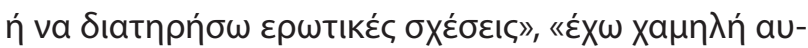

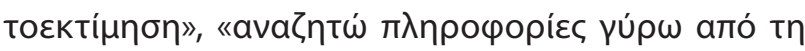

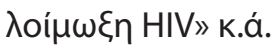

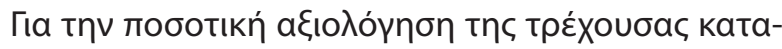

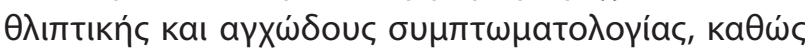

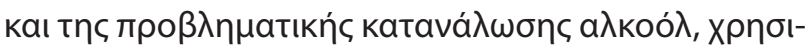

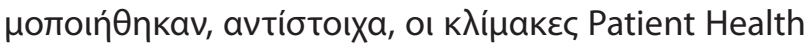
Questionnaire (PHQ-927,28), Beck Anxiety Inventory

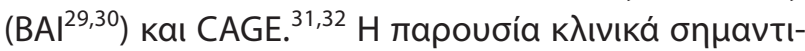

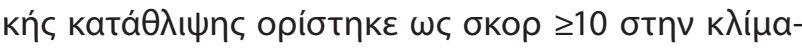

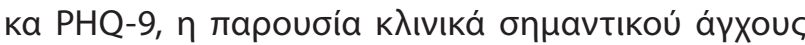

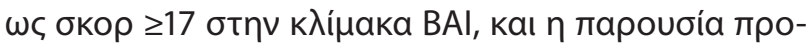

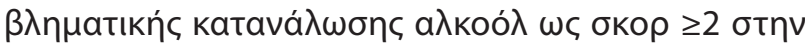

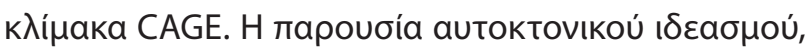

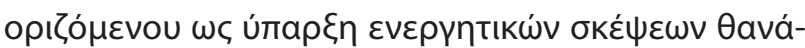

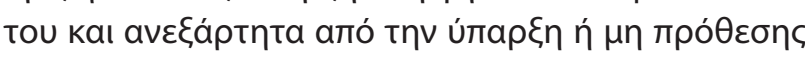

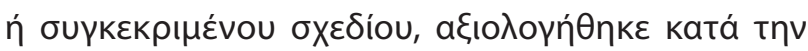

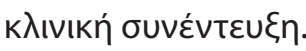

\section{Avánuø耳}

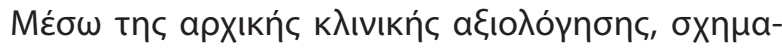

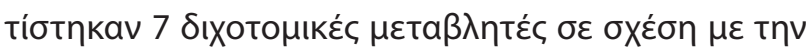

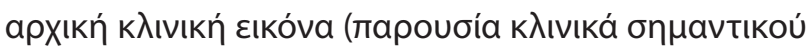

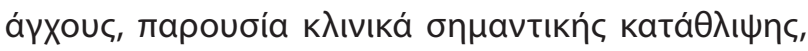

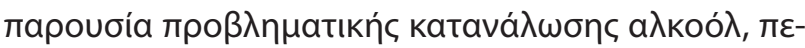

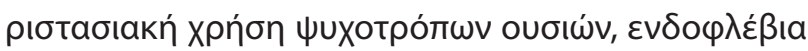

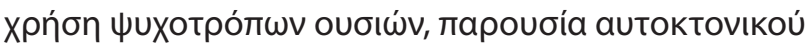

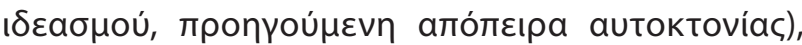

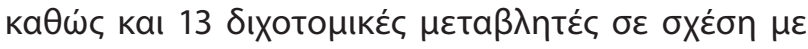

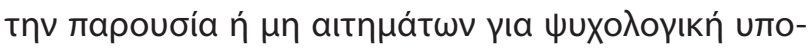

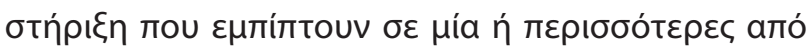

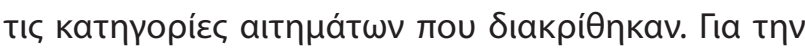

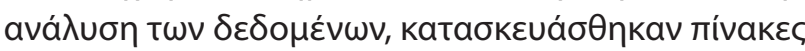

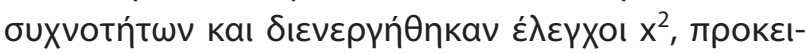

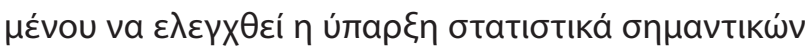

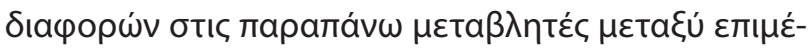

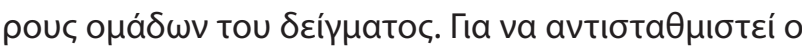

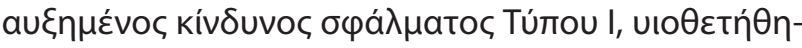

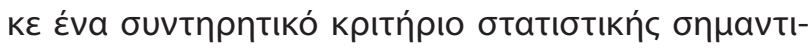

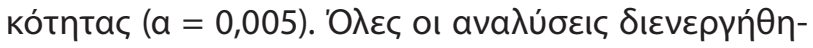

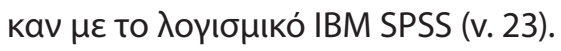

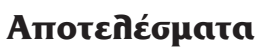

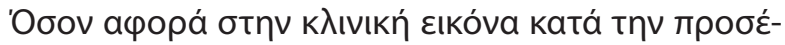

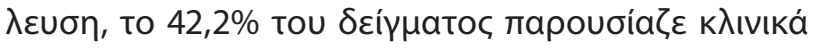

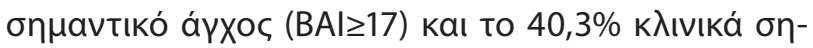

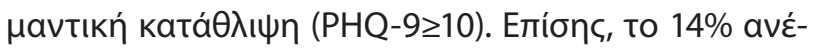

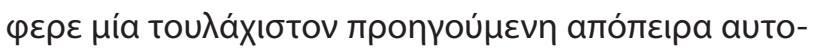

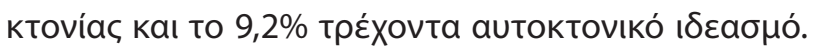

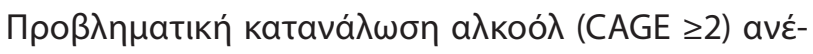

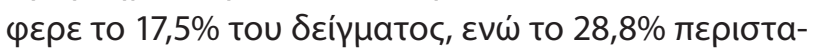

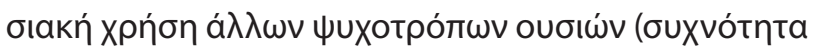

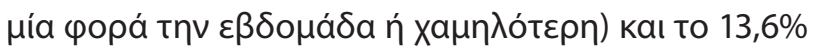

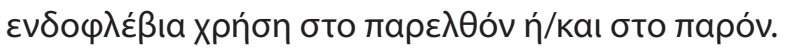

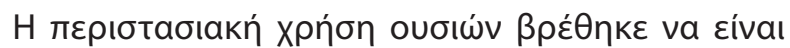

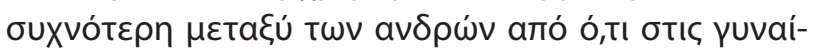

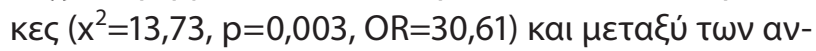

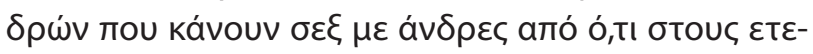

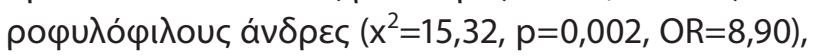

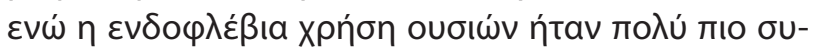

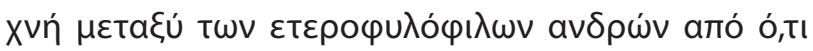

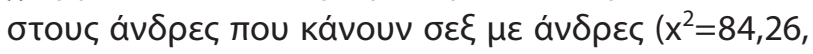

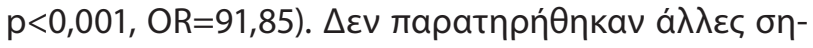

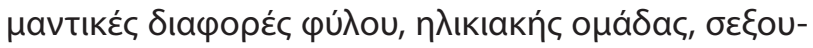

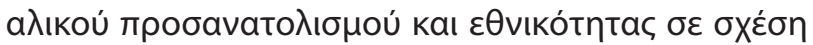

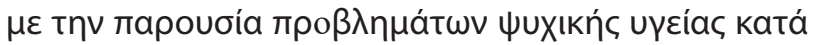

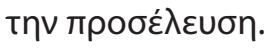

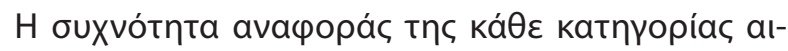

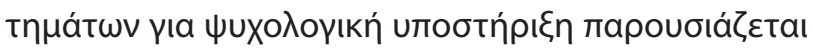

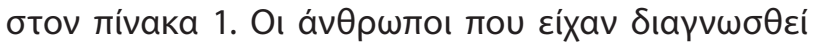

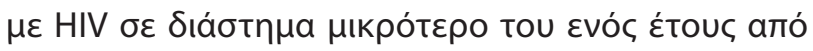

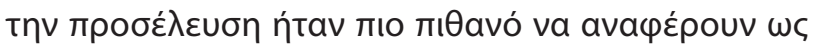

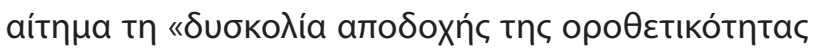

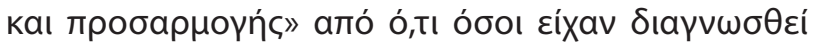

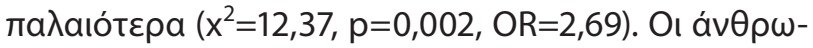

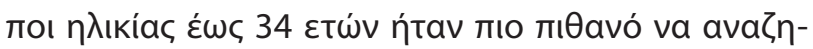

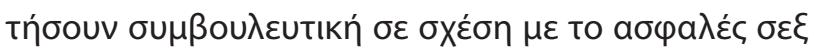

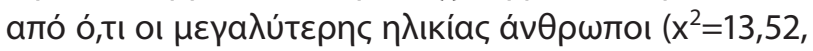

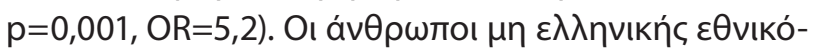

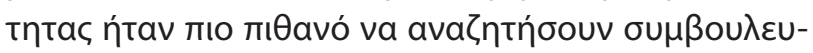

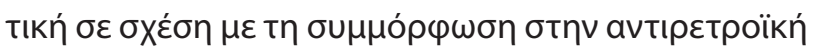

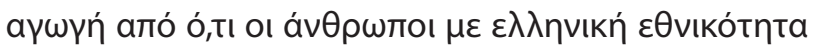

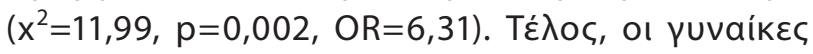

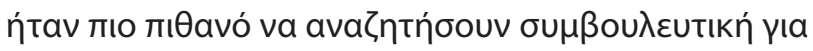

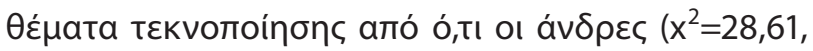




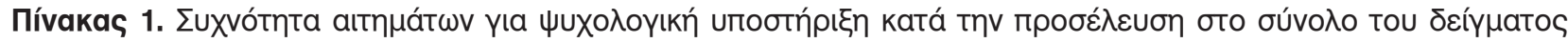
$(\mathrm{N}=191)$.

\begin{tabular}{|c|c|c|}
\hline 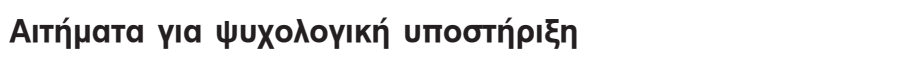 & $\mathbf{n}$ & (\%) \\
\hline 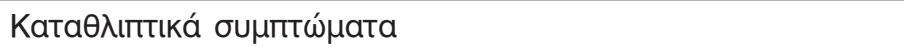 & 112 & $58,6 \%$ \\
\hline 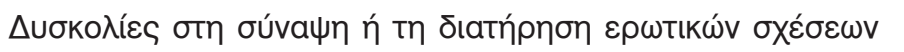 & 93 & $48,7 \%$ \\
\hline 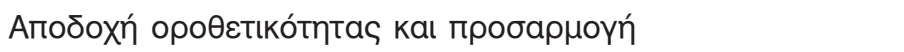 & 82 & $42,9 \%$ \\
\hline 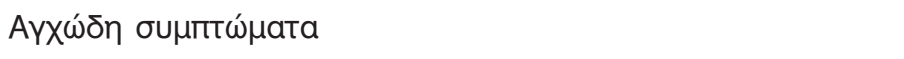 & 81 & $42,4 \%$ \\
\hline 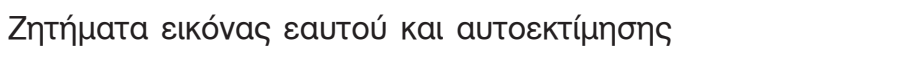 & 78 & $40,8 \%$ \\
\hline 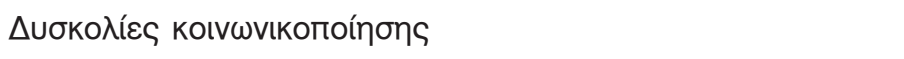 & 69 & $36,1 \%$ \\
\hline 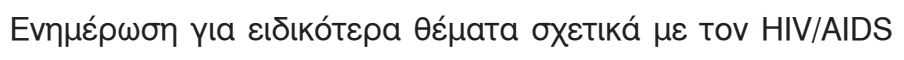 & 64 & $33,5 \%$ \\
\hline 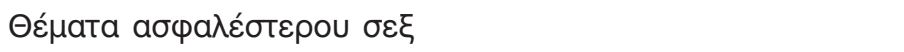 & 30 & $15,7 \%$ \\
\hline 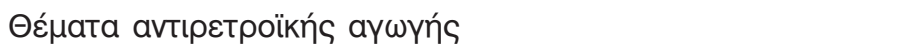 & 11 & $5,8 \%$ \\
\hline 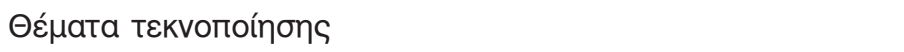 & 9 & $4,7 \%$ \\
\hline 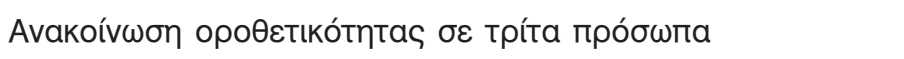 & 60 & $31,4 \%$ \\
\hline 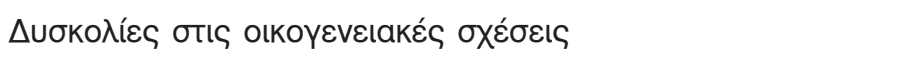 & 51 & $26,7 \%$ \\
\hline 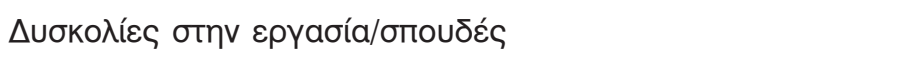 & 39 & $20,4 \%$ \\
\hline 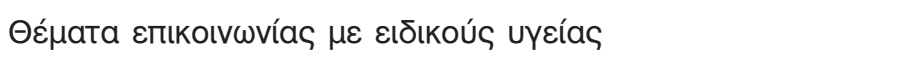 & 33 & $17,3 \%$ \\
\hline 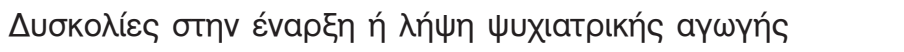 & 24 & $12,6 \%$ \\
\hline 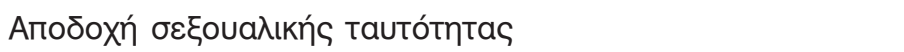 & 23 & $12,0 \%$ \\
\hline
\end{tabular}

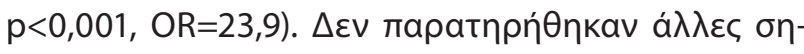

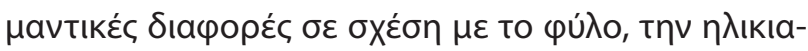

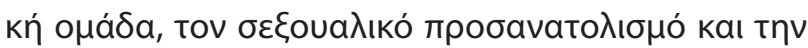

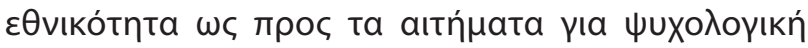

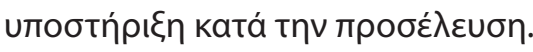

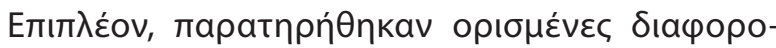

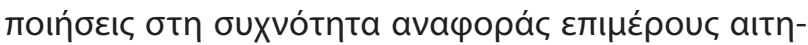

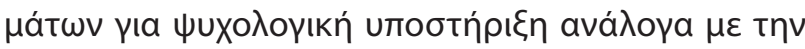

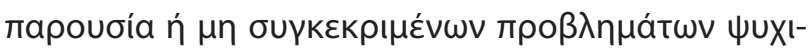

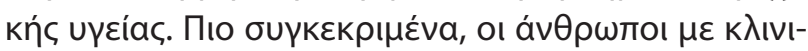

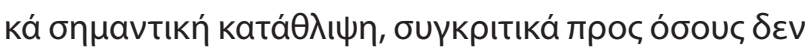

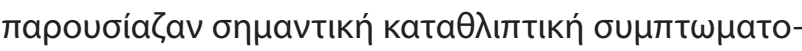

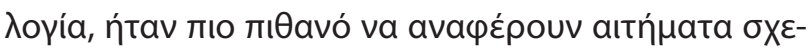

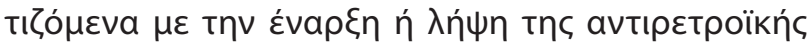

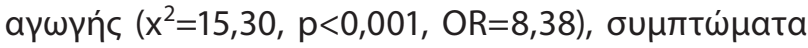

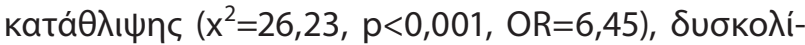

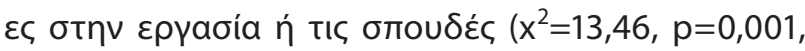

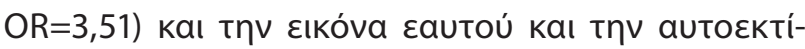

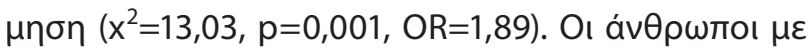

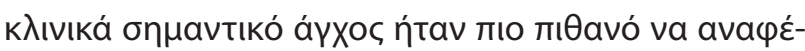

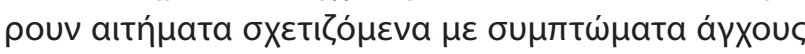
$\left(x^{2}=71,99, p<0,001, O R=19,43\right)$, $\varepsilon v \omega \dot{~ o l ~ a ́ v \theta \rho \omega \pi о ~} \mu \varepsilon$

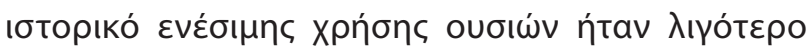

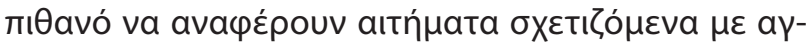

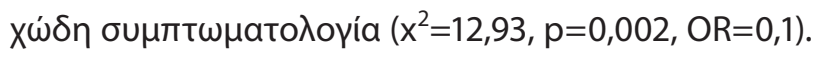

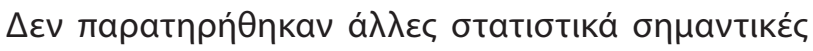

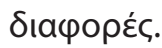

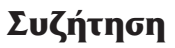

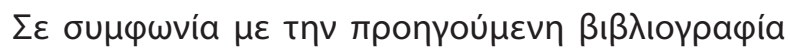

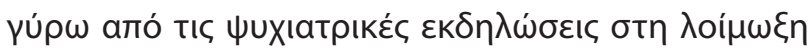

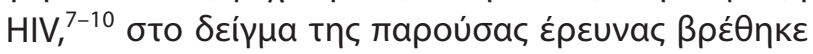

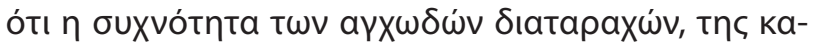

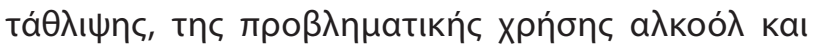

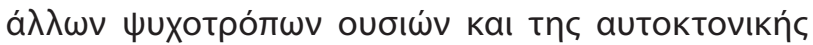

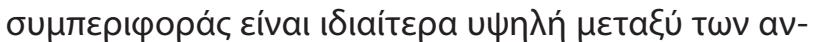

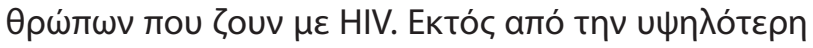

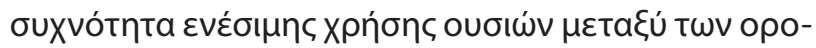

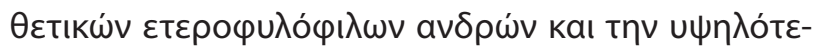

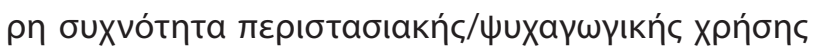

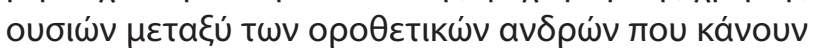

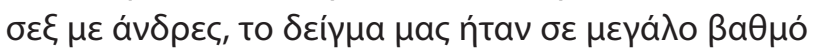

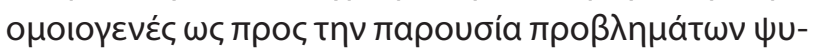

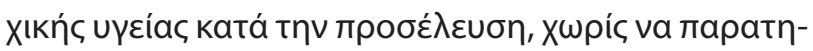

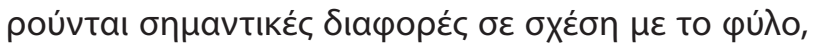

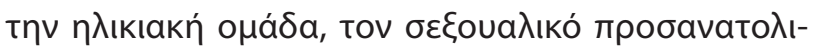

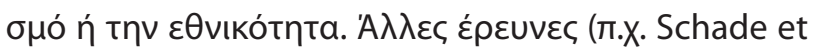

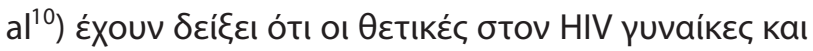




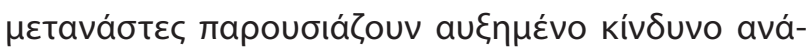

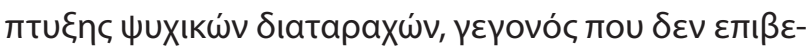

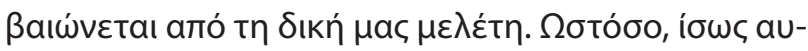

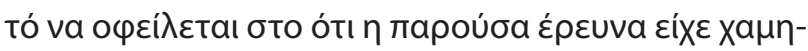

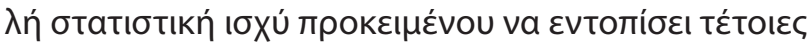

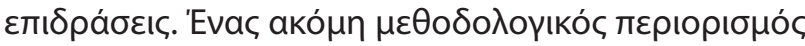

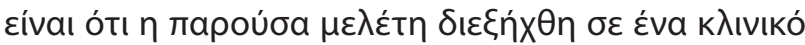

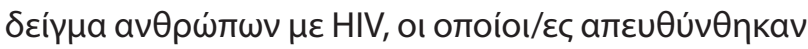

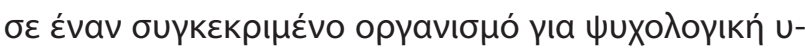

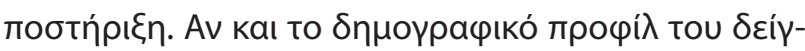

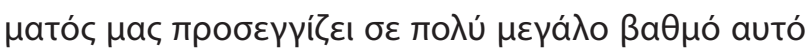

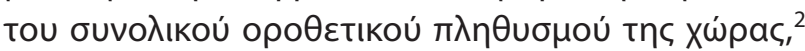

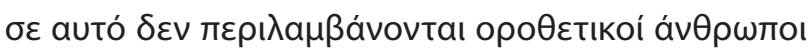

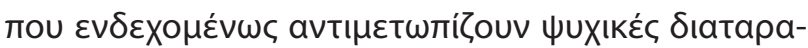

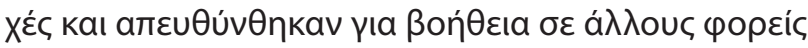

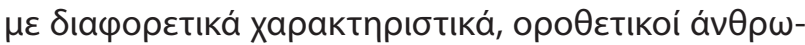

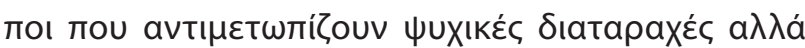

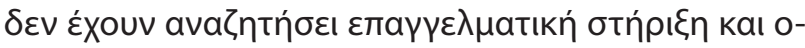

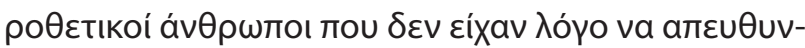

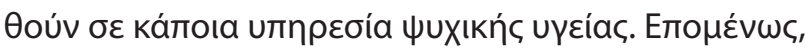

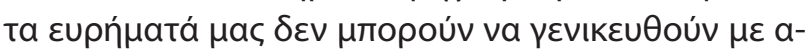

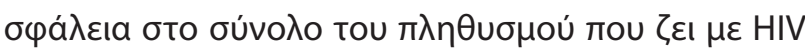
$\sigma \tau \eta v E \lambda \lambda a^{\prime} \delta a$.

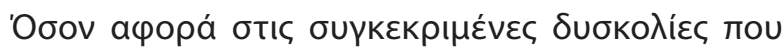

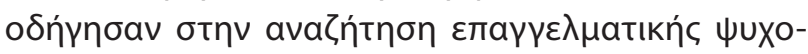

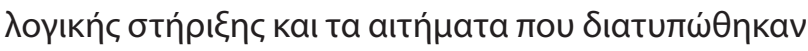

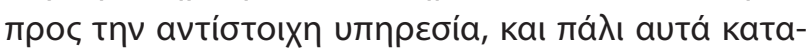

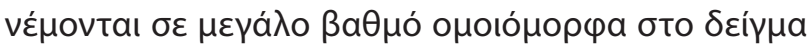

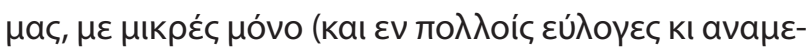

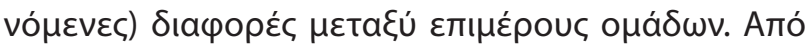

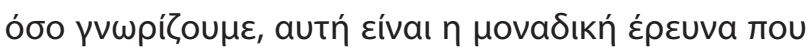

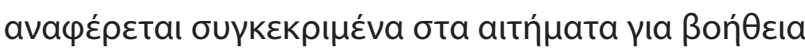

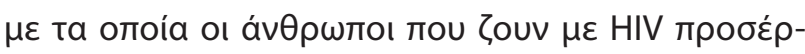

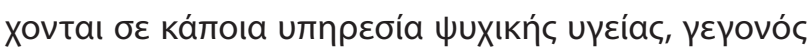

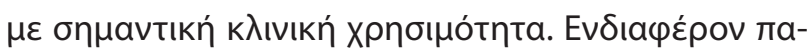

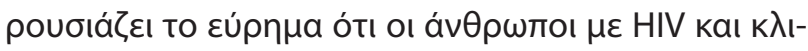

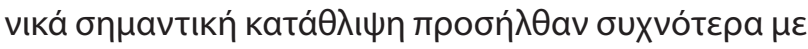

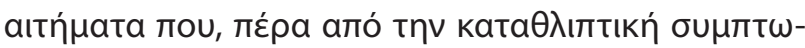

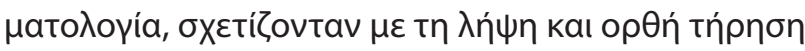

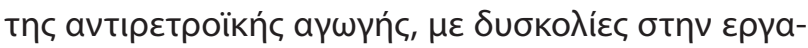

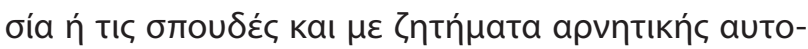

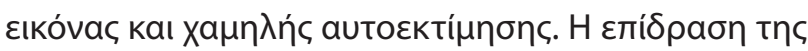

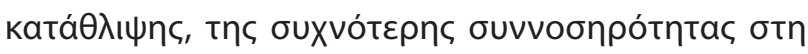

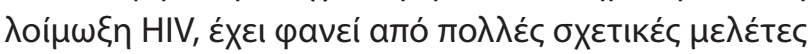

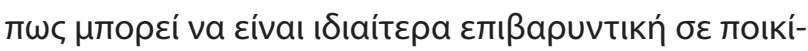

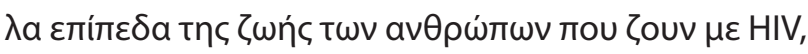

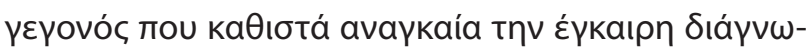

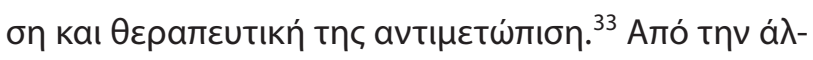

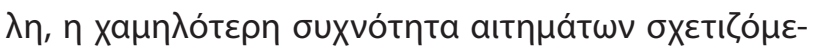

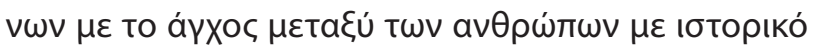

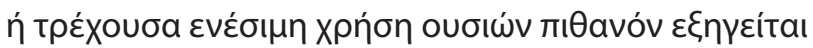

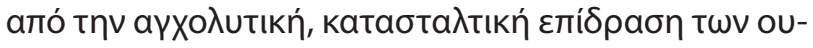

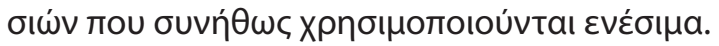

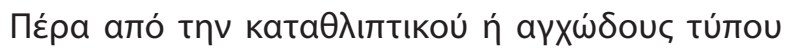

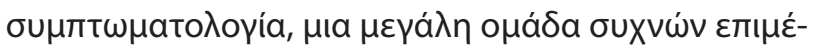

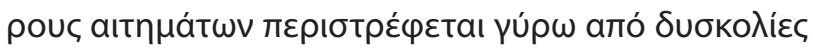

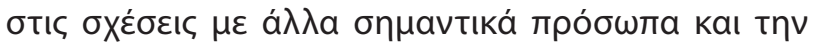

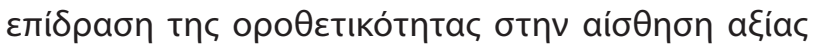

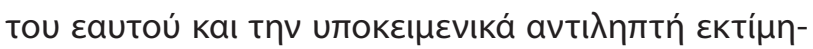

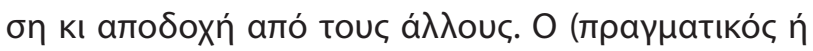

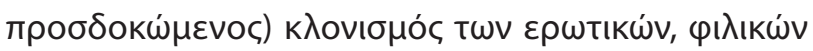

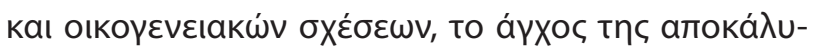

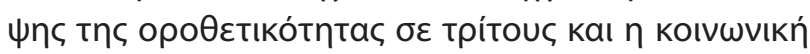

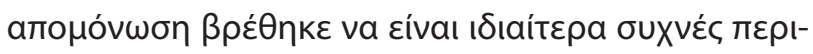

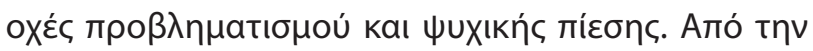

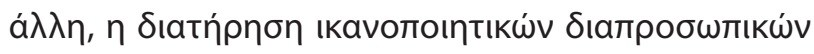

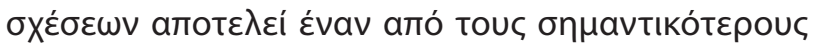

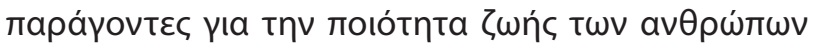

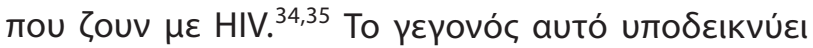

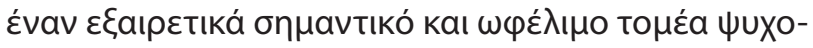

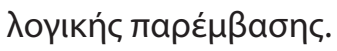

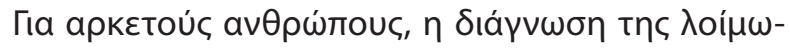

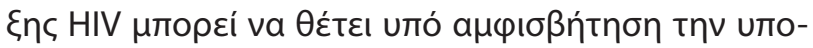

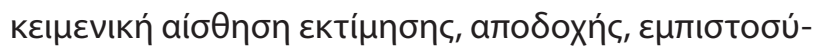

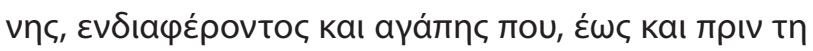

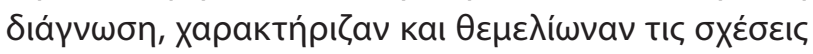

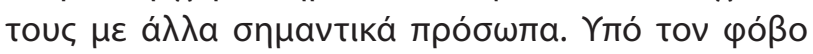

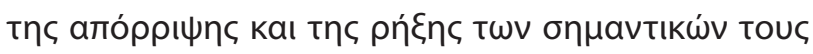

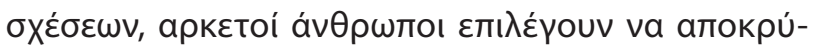

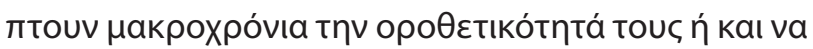

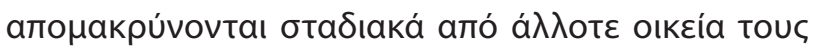

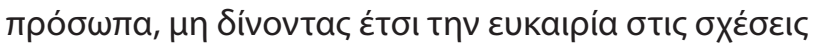

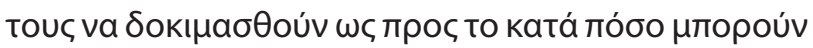

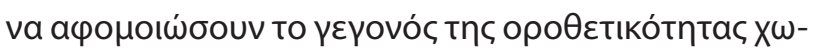

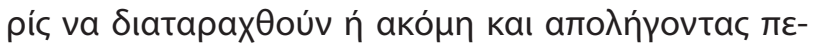

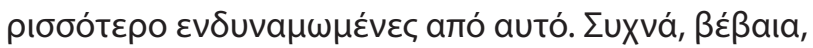

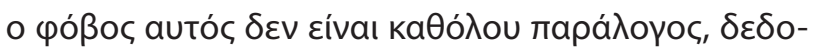

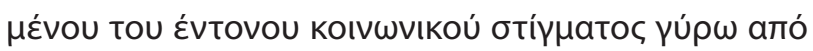

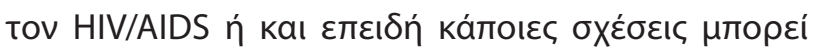

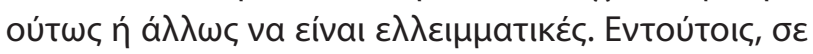

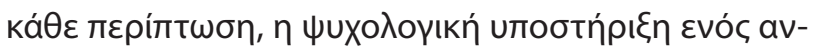

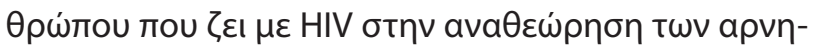

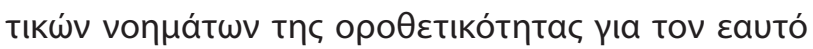

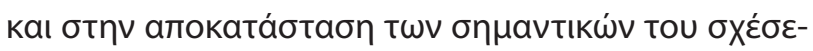




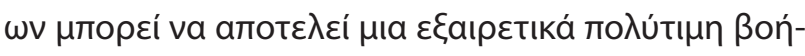

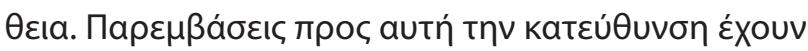

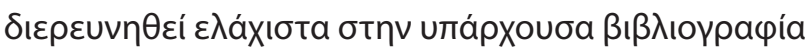

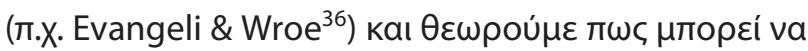

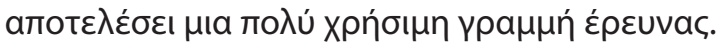

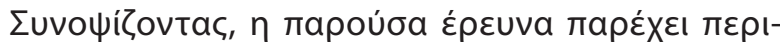

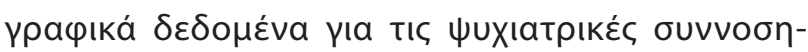

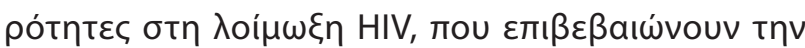

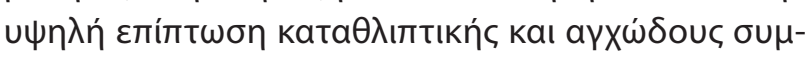

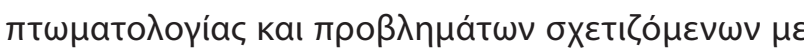

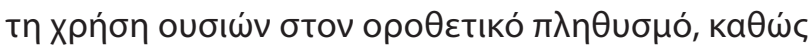

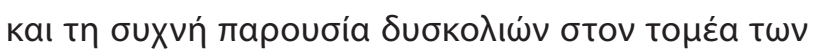

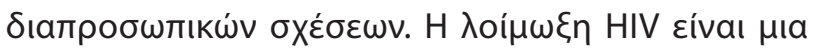

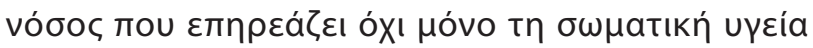

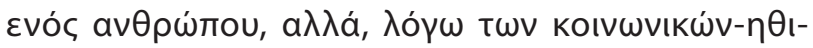

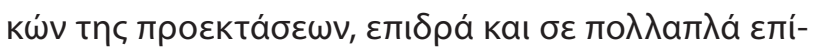

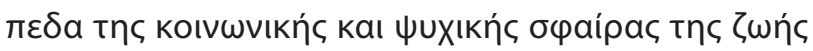

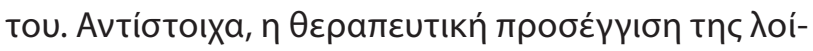

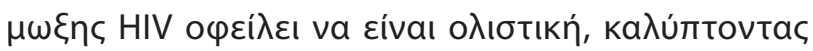

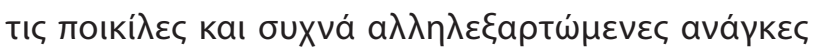

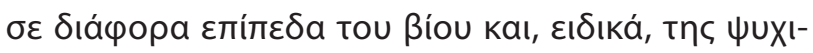
kńc uүعíac.

\title{
Clinical presentation and requests for support of people with HIV/AIDS seeking counselling and psychotherapy in Greece
}

\author{
A. Peltekis, ${ }^{1}$ M. Koulentianou ${ }^{2}$ \\ ${ }^{1}$ Centre for Life, Thessaloniki, ${ }^{2}$ Centre for Life, Athens, Greece
}

Psychiatriki 2019, 30:120-128

Despite the large progress during the last decades in the medical treatment of HIV/AIDS infection, people living with HIV nevertheless face multiple adversities at various levels of their lives. Mental disorders, in particular, are the most common comorbidities in HIV infection with negative consequences in adherence to antiretroviral medication, disease progress and overall quality of life. HIVrelated stigma, still quite intense in Greece, is one of the most debilitating factors concerning people's living with HIV mental health. The present study looked at the clinical presentation at intake and treatment requests of 191 (83\% males) people living with HIV who addressed the psychological support service of the non-governmental organization 'Centre for Life' during the years 201618. Data were collected through a semi-structured clinical interview and administration of adapted questionnaires (PHQ-9, BAI, CAGE), which resulted in 7 dichotomous variables related to clinical presentation and 13 dichotomous variables related to treatment requests at intake. To analyze data, we constructed frequency tables and performed chi-square tests. In the whole sample, $42.2 \%$ presented anxiety disorders, $40.3 \%$ depression, $28.8 \%$ occasional substance use, $17.5 \%$ problematic use of alcohol and $13.6 \%$ intravenous drug use. Moreover, $14 \%$ reported at least one suicide attempt in the past and 9.2\% current suicidal ideation. Apart from more frequent intravenous drug use among heterosexual males and more frequent occasional/recreational drug use among men who have sex with men, no other differences related to gender, age group, sexual orientation or ethnicity were observed in the initial clinical presentation. Similarly, the most frequent treatment requests were homogenously distributed in our sample, such as depressive symptoms (58.6\%), difficulties in romantic relationships (48.7\%), accepting being HIV positive (42.9\%), anxiety symptoms (42.4\%) and issues of negative self-esteem (40.8\%). The presence of clinically significant depression was found to be related to a wider range of treatment requests compared to other mental health problems. A large group 
of requests focused on interpersonal relationships difficulties (e.g. disclosure anxiety, social isolation, disturbed relationships with familiar persons). This indicates an important area of psychological intervention. HIV infection may affect many levels of an individual's life, including their mental health. Respectively, HIV treatment needs to adopt a more holistic approach.

Key words: HIV/AIDS infection, mental health problems, requests for psychological support, interpersonal relationships.

\section{Bıßntoypacpía}

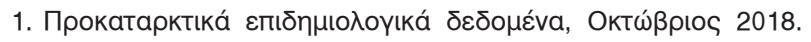

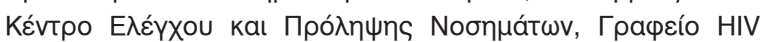

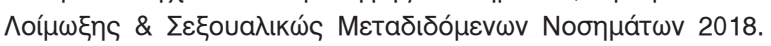

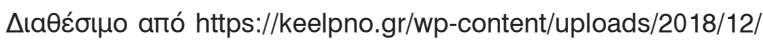
epidimiologiko-31-10-2018_final.pdf

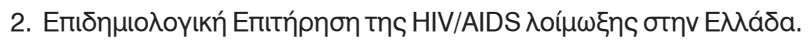

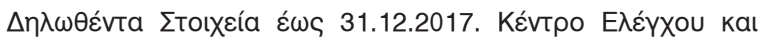

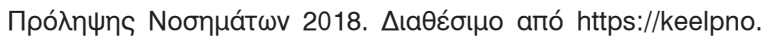
gr/wp-content/uploads/2018/12/epidimiologiko-2017.pdf

3. The Antiretroviral Cohort Collaboration. Survival of HIV-positive patients starting antiretroviral therapy between 1996 and 2013: a collaborative analysis of cohort studies. Lancet HIV 2017, 4:e349-e356, doi: 10.1016/S2352-3018(17)30066-8

4. Rodger A, Cambiano V, Bruun T, Vernazza P, Collins S, Corbelli GM et al. Risk of HIV transmission through condomless sex in MSM couples with suppressive ART: The PARTNER2 Study extended results in gay men. Профорıки́ пароuбíaon бт 220

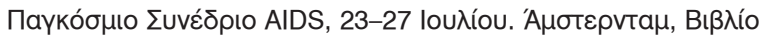

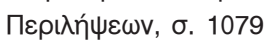

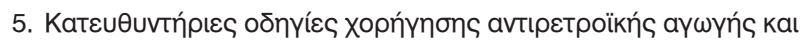

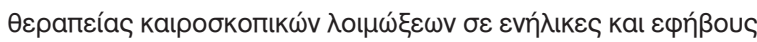

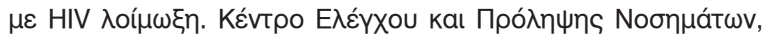

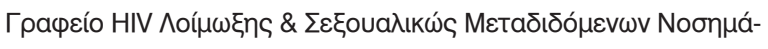

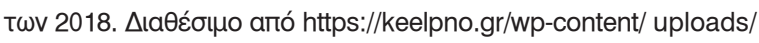
2018/12/antiretroiki-agogi-11-05-2018.pdf

6. Vourli G, Nikolopoulos G, Paparizos V, Skoutelis A, Metallidis S, Gargalianos P et al. HIV cascade of care in Greece: useful insights from additional stages. PLoS One 2018, 13: e0207355, doi: 10.1371/journal.pone.0207355

7. Adams C, Zacharia S, Masters L, Coffey C, Catalan P. Mental health problems in people living with HIV: changes in the last two decades: the London experience 1990-2014. AIDS Care 2016, 28:56-59, doi: 0.1080/09540121.2016.1146211

8. Kendall CE, Wong J, Taljaard M, Glazier RH, Hogg W, Younger $\mathrm{J}$ et al. A cross-sectional, population-based study measuring comorbidity among people living with HIV in Ontario. BMC Public Health 2014, 14:161, doi: 10.1186/1471-2458-14-161

9. Edmiston N, Passmore E, Smith DJ, Petoumenos K. Multimorbidity among people with HIV in regional New South Wales, Australia. Sex Health 2015, 12:425-432, doi: 10.1071/SH14070

10. Schadı A, van Grootheest G, Smit JH. HIV-infected mental health patients: characteristics and comparison with HIV-infected patients from the general population and non-infected mental health patients. BMC Psychiatry 2013, 13:35, doi: 10.1186/1471244X-13-35
11. Holzemer WL, Human S, Arudo J, Rosa ME, Hamilton MJ, Corless I et al. Exploring HIV stigma and quality of life for persons living with HIV infection. J Assoc Nurses AIDS Care 2009, 20:161-168, doi: 10.1016/j.jana.2009.02.002

12. Fuster-Ruizdeapodaca MJ, Molero F, Holgado FP, Mayordomo S. Enacted and internalized stigma and quality of life among people with HIV: the role of group identity. Qual Life Res 2014, 23:1967-1975, doi: 10.1007/s11136-014-0653-4

13. Gesesew HA, Gebremedhin AT, Demissie TD, Kerie MW, Sudhakar M, Mwanri L. Significant association between perceived HIV related stigma and late presentation for HIV/AIDS care in low and middle-income countries: A systematic review and meta-analysis. Qual Life Res 2014, 23:1967-1975, doi: 10.1371/journal.pone. 0173928

14. Katz IT, Ryu AE, Onuegbu AG, Psaros C, Weiser SD, Bangsberg DR et al. Impact of HIV-related stigma on treatment adherence: systematic review and meta-synthesis. J Int AIDS Soc 2013, 16:18640, doi: 10.7448/IAS.16.3.18640

15. Jallow A, Ljunggren $G$, Wändell $P$, Wahlström L, Carlsson AC. HIV-infection and psychiatric illnesses - A double edged sword that threatens the vision of a contained epidemic: The Greater Stockholm HIV Cohort Study. J Infect 2016, 74:22-28, doi: 10.1016/j.jinf.2016.09.009

16. Rueda S, Mitra S, Chen S, Gogolishvili D, Globerman J, Chambers $L$ et al. Examining the associations between HIVrelated stigma and health outcomes in people living with HIV/ AIDS: a series of meta-analyses. BMJ Open 2016, 6:e011453, doi: 10.1136/bmjopen-2016-011453

17. Vagenas P, Azar MM, Copenhaver MM, Springer SA, Molina $\mathrm{PE}$, Altice FL. The impact of alcohol use and related disorders on the HIV continuum of care: a systematic review. Curr HIV/ AIDS Rep 2015, 12:421-436, doi: 10.1007/s11904-015-0285-5

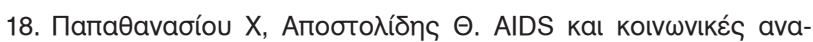

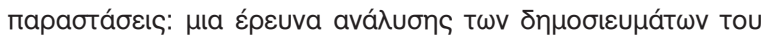

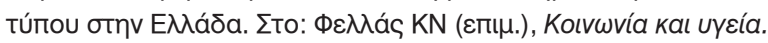

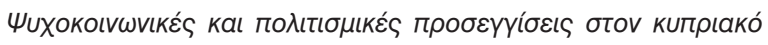

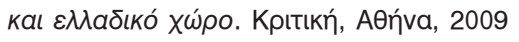

19. Kalemi G, Gkioka S, Tsapatsari P, Tzeferakos G, Kandri T, Psarra ML. Stigma and self-esteem: a case of HIV-positive sex-workers. Psychiatriki 2017, 28:67-74, doi: 10.22365/jpsych.2017.281.67

20. The Greek Association of PLHIV - Positive Voice. The people

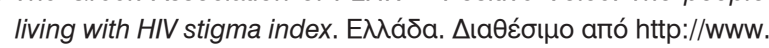
stigmaindex.org/sites/default/files/reports/Stigmalndex_Greece_ Final_ENG.pdf 


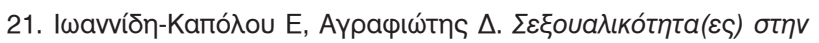

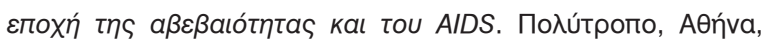
2005

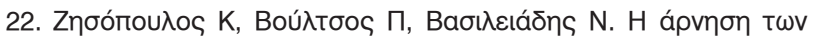

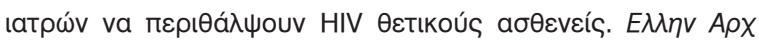
AIDS 2010, 18:54-60

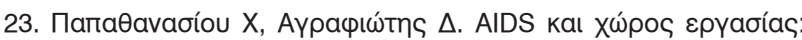

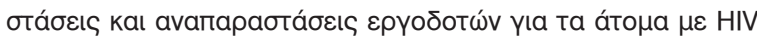

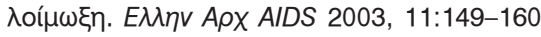

24. Paparizos V, Triantafyllopoulou I, Kourkounti S, Retsas T, Paparizou E. Suicidal behaviour in HIV-infected patients in Greece. Infez Med 2017, 25:64-70, PMID: 28353458

25. Ginieri-Coccossis M, Triantafillou E, Papanikolaou N, Baker R, Antoniou C, Skevington SM et al. Quality of life and depression in chronic sexually transmitted infections in UK and Greece: the use of WHOQOL-HIV/STI BREF. Psychiatriki 2018, 29:209-219, doi: $10.22365 /$ jpsych.2018.293.209

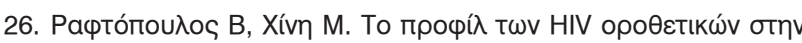

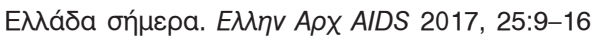

27. Kroenke K, Spitzer RL, Williams JB. The PHQ-9: validity of a brief depression severity measure. J Gen Intern Med 2001, 16:606613, PMID: 11556941

28. Karekla M, Pilipenko N, Feldman J. Patient Health Questionnaire: Greek language validation and subscale factor structure. Compr Psychiatry 2012, 53:1217-1226, doi: 10.1016/j.comppsych.2012.05.008

29. Beck AT, Epstein N, Brown G, Steer RA. An inventory for measuring clinical anxiety: psychometric properties. J Consult Clin Psychol 1988, 56:893-897, doi: 10.1037/0022-006X.56.6.893

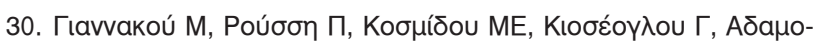

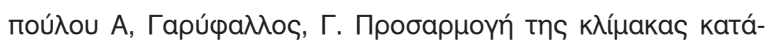

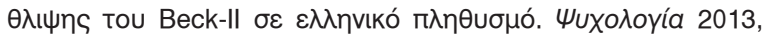
$10: 120-146$
31. Ewing JA. Detecting alcoholism. The CAGE questionnaire. JAMA 1984, 252:1905-1907, PMID: 6471323

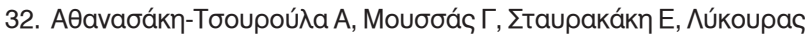

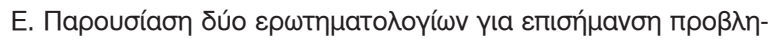

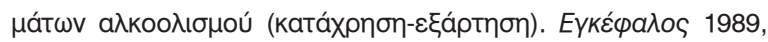
26:71-74

33. Nanni MG, Caruso R, Mitchell AJ, Meggiolaro E, Grassi L. Depression in HIV infected patients: a review. Curr Psychiatry Rep 2015, 17:530, doi: 10.1007/s11920-014-0530-4

34. Degroote S, Vogelaers D, Vandijck DM. What determines health-related quality of life among people living with HIV: an updated review of the literature. Arch Publ Health 2014, 72:40, doi: 10.1186/2049-3258-72-40

35. Drewes J, Gusy B, Róden U. More than 20 years of research into the quality of life of people with HIV and AIDS: a descriptive review of study characteristics and methodological approaches of published empirical studies. J Int Assoc Provid AIDS Care 2013, 12:18-22, doi: 10.1177/1545109712456429

36. Evangeli M, Wroe AL. HIV disclosure anxiety a systematic review and theoretical synthesis. AIDS Behav 2017, 21:1-11, doi: 10.1007/ s10461-016-1453-3

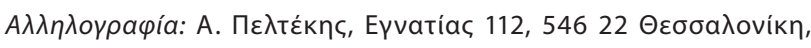
$\operatorname{T\eta } \lambda$ : 2310-23 70 40, e-mail: a.peltekis@kentrozois.gr 


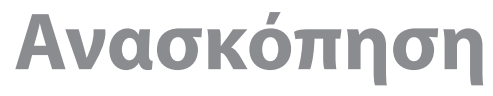 Review
}

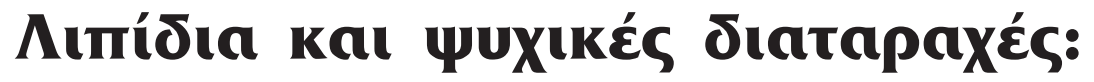

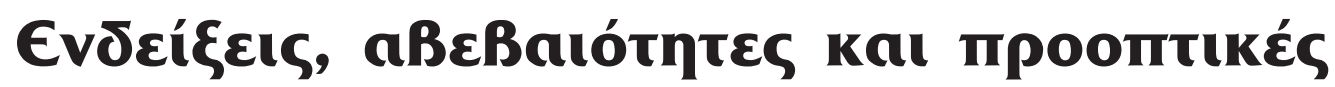

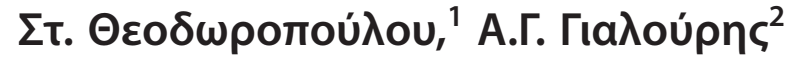

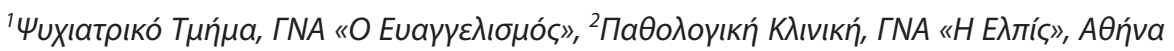

uuxıatpıkń 2019, 30:129-141

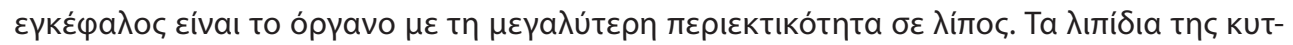

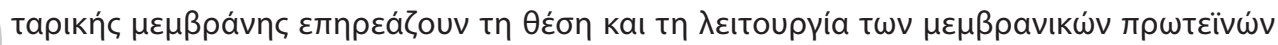

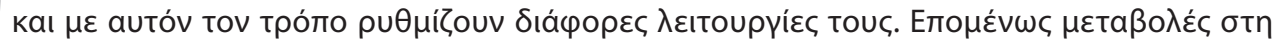

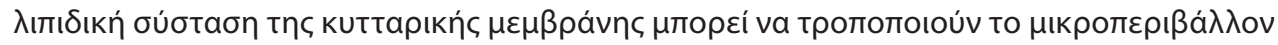

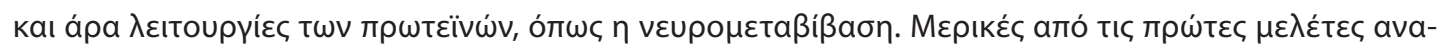

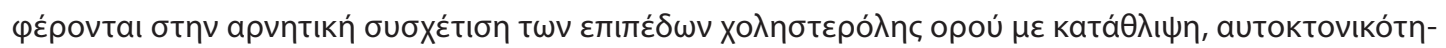

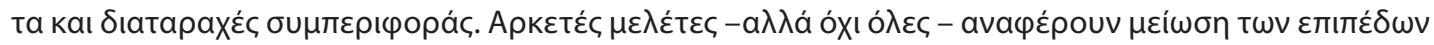

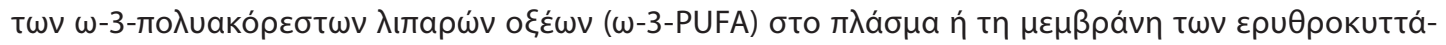

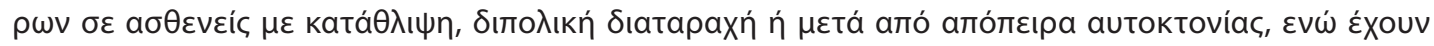

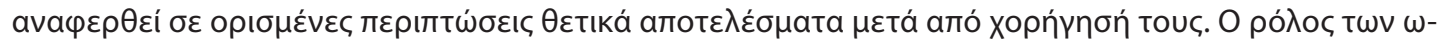

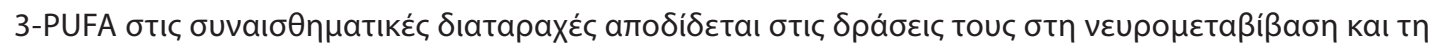

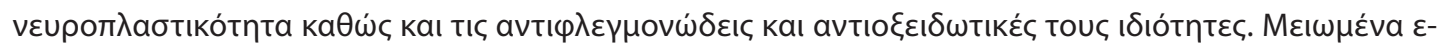

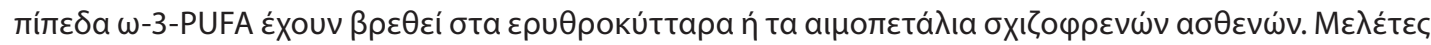

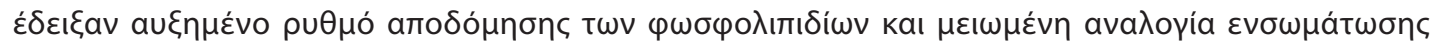

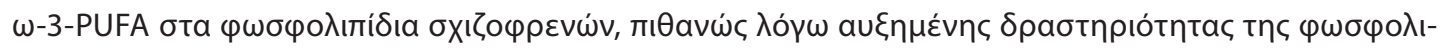

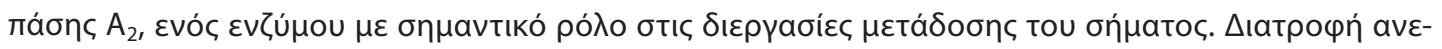

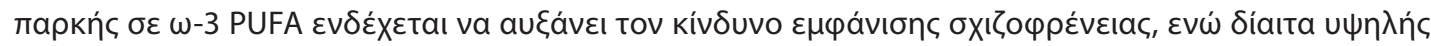

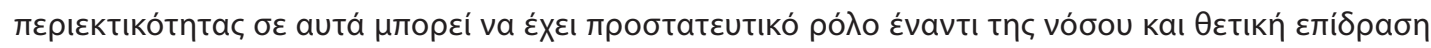

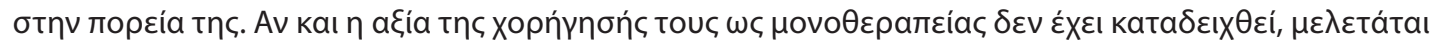

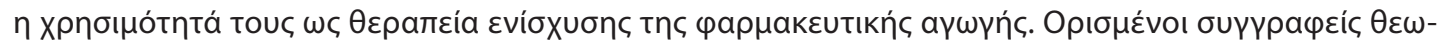

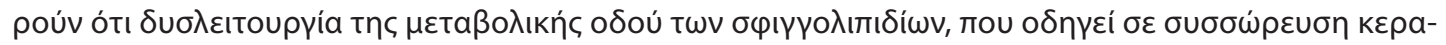

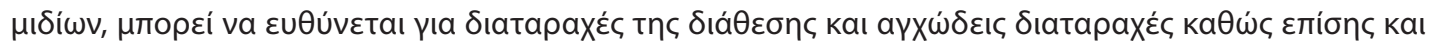




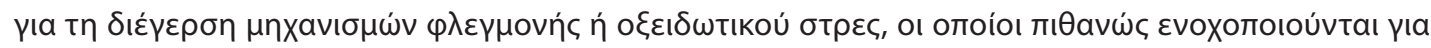

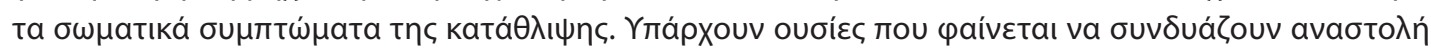

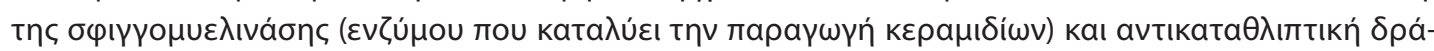

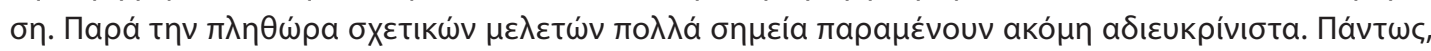

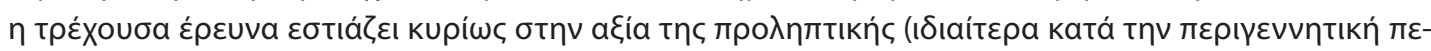

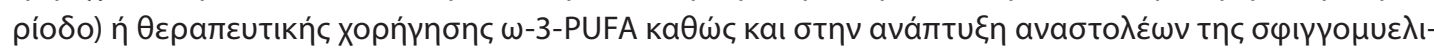

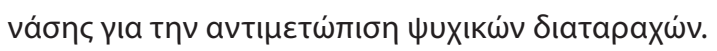

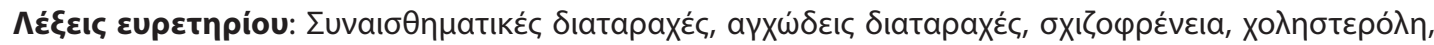

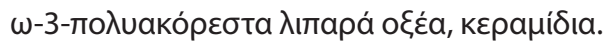

\section{Eıбaywyń}

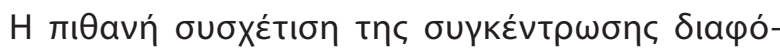

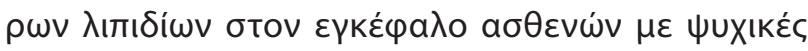

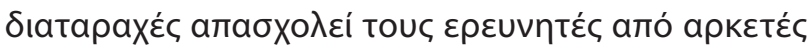

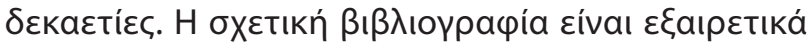

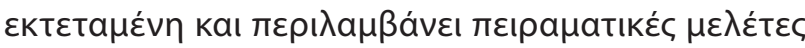

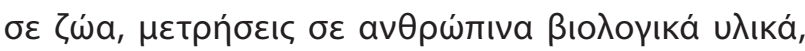

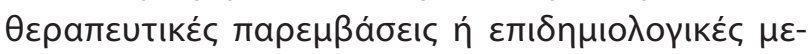

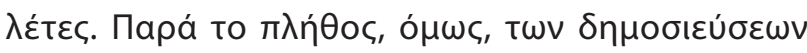

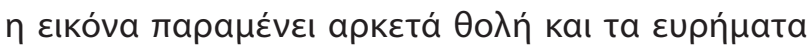

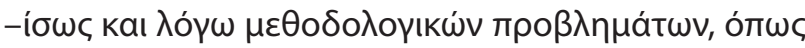
$\mu$ нкро́ ар

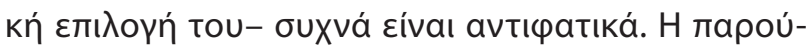

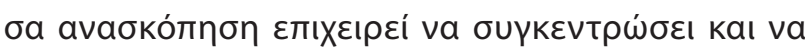

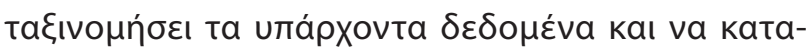

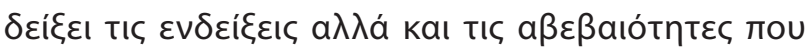

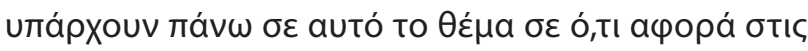

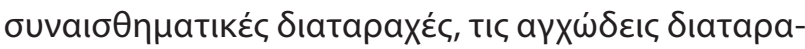

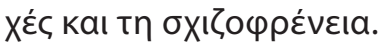

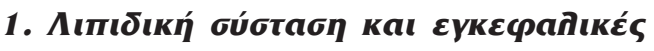

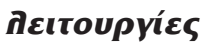

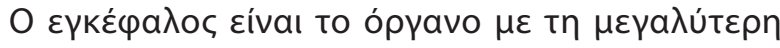

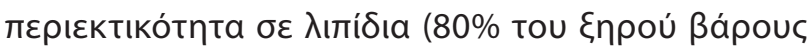

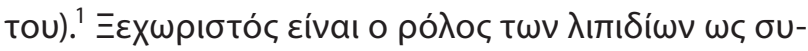

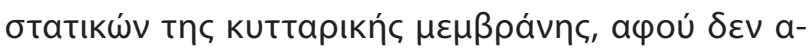

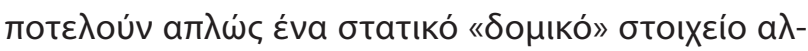

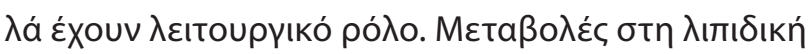

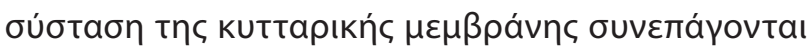

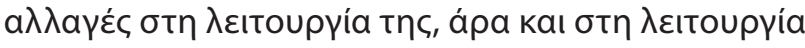

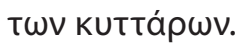

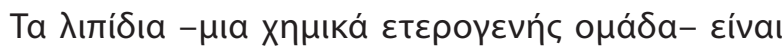

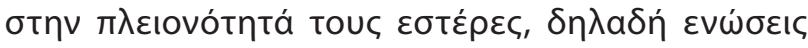

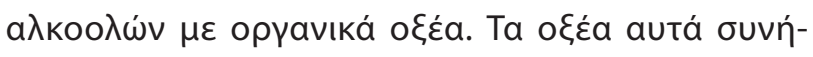

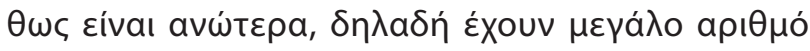

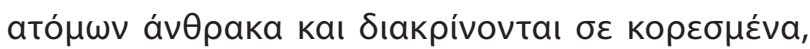

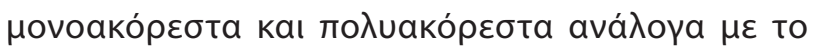

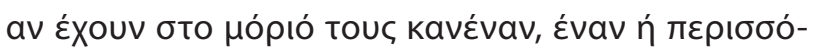

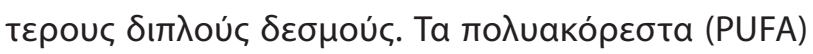

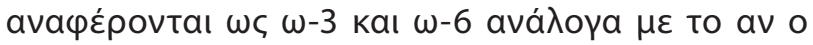

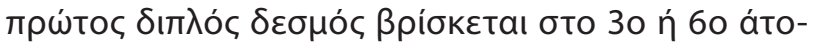

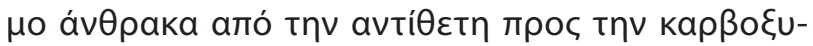

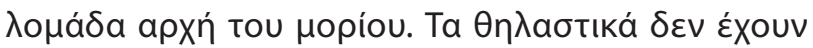

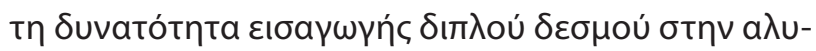

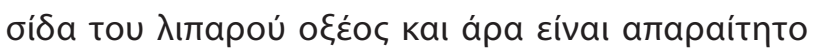

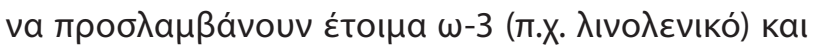

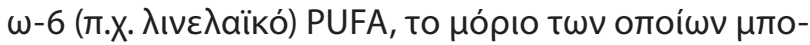

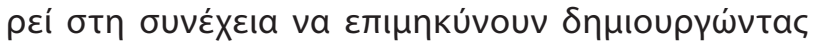

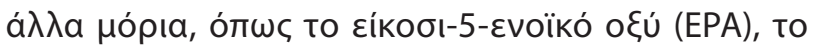

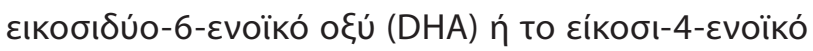

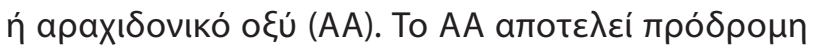

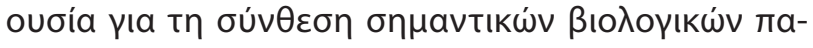

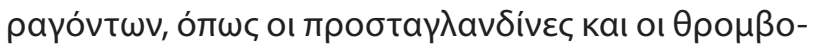
Łávec.

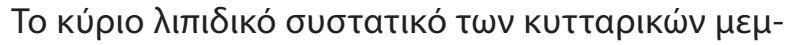

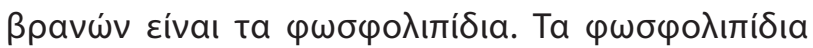

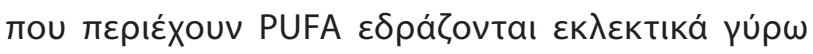

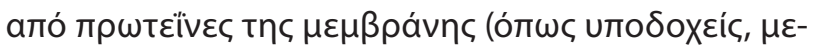

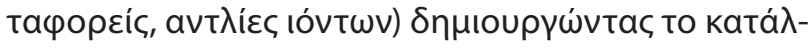

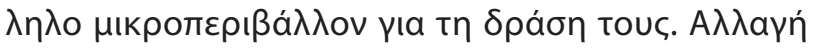

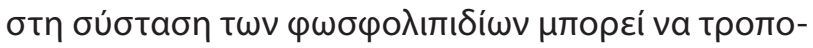

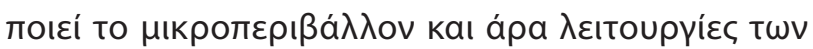

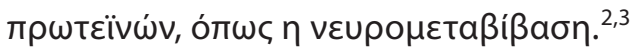

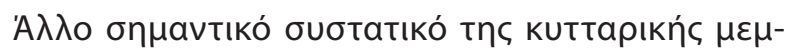

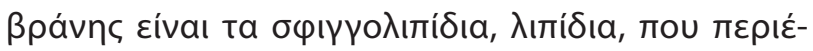

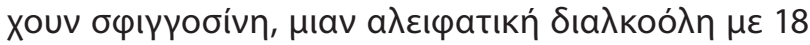




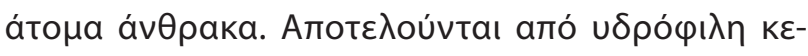

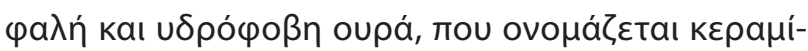

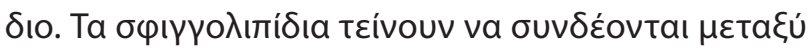

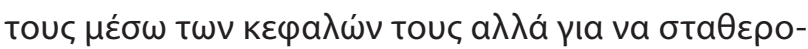

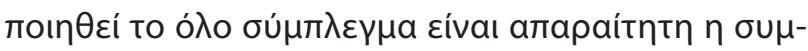

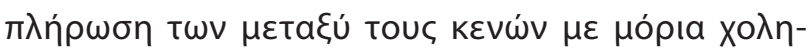

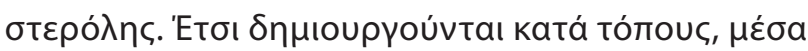

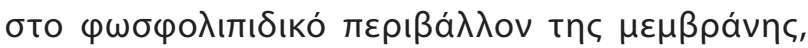

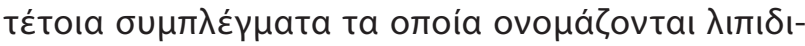

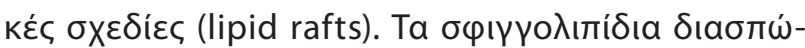

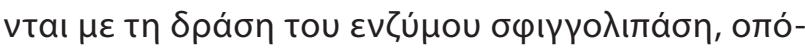

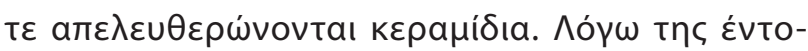

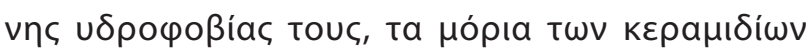

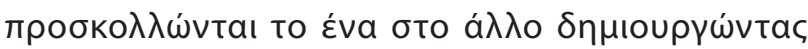

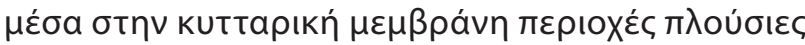

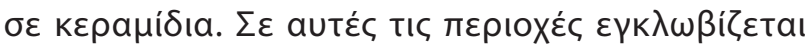

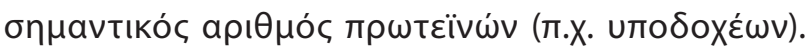

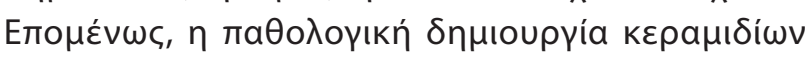

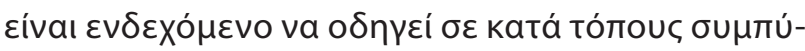

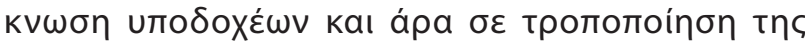
$\mu \varepsilon \mu \beta$ pavikńc $\lambda \varepsilon$ เтouppíac. ${ }^{4}$

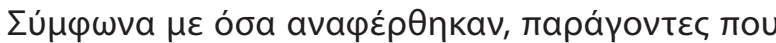

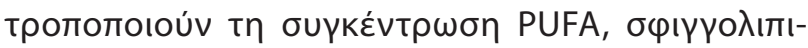

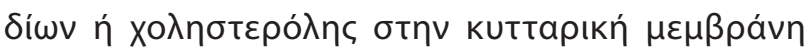

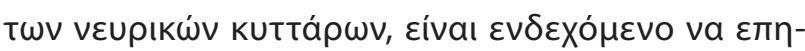

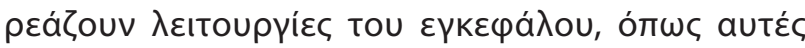

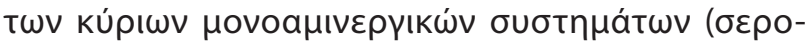

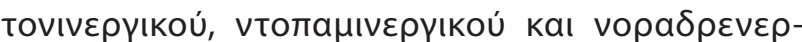

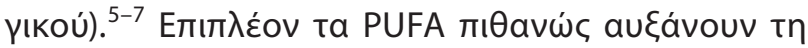

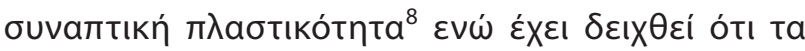

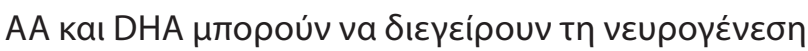
бтоv เтто́канто., ${ }^{9,10}$

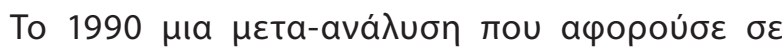

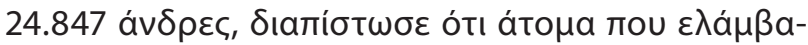

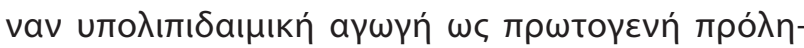

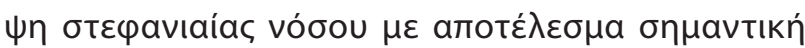

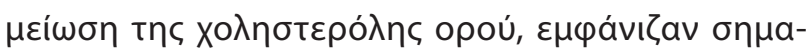

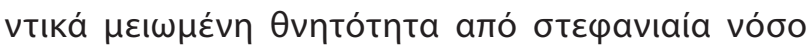

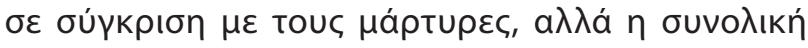

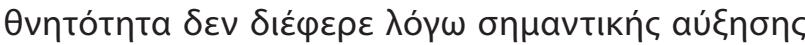

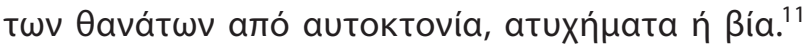

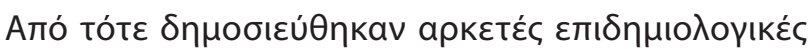
$\mu \varepsilon \lambda \varepsilon \dot{t \varepsilon \varsigma, ~ о І ~ о т о і ́ \varepsilon \varsigma ~ a v a \varphi \varepsilon ́ p o v t a ı ~ \sigma т \eta ~ \sigma X \varepsilon ́ \sigma \eta ~ б U ү к \varepsilon-~}$

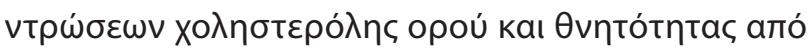

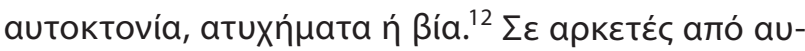

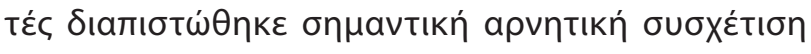

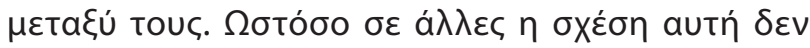
$\varepsilon \pi ı \varepsilon \beta а ı \dot{\theta \eta п к \varepsilon . ~}{ }^{13}$

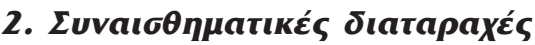

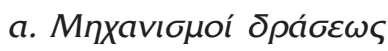

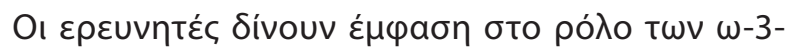

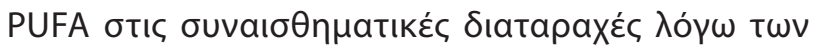

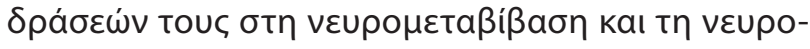

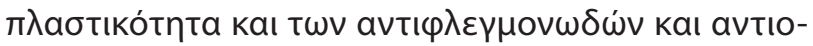

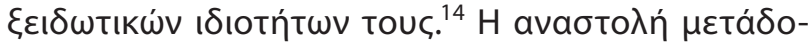

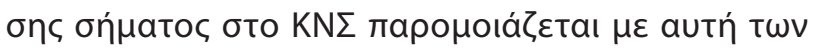

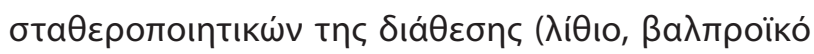

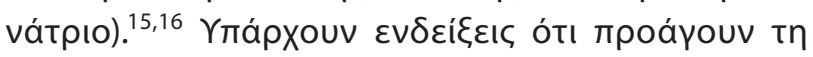

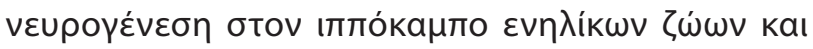

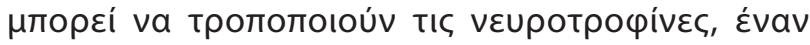

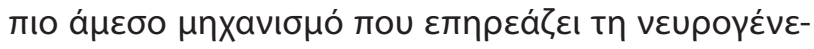
on. ${ }^{17-19}$

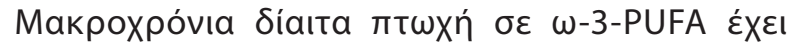

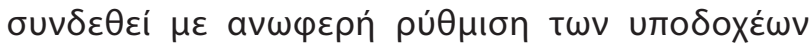

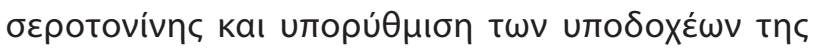

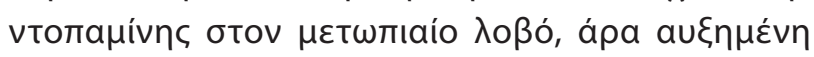

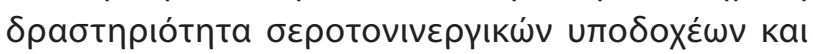

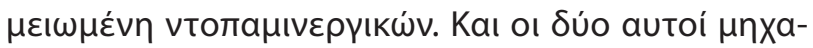

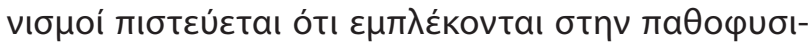

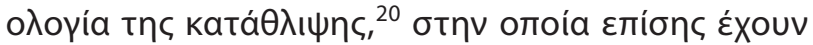

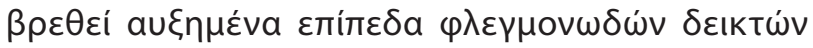

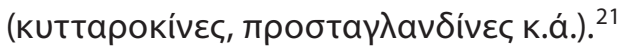

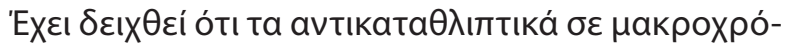

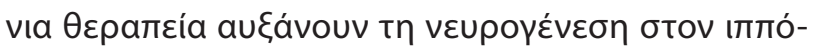

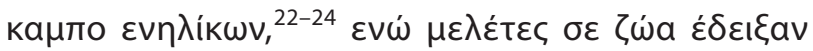

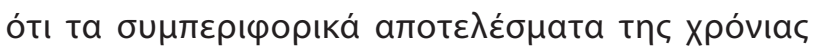

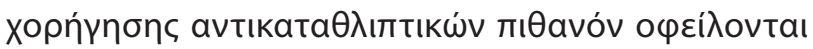

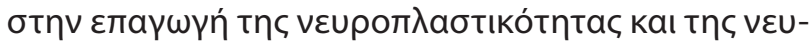

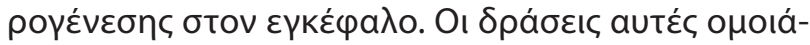

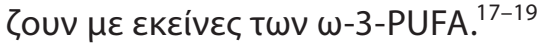

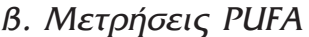

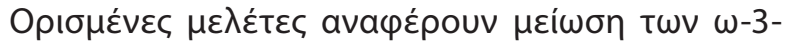

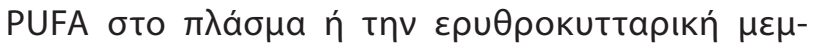

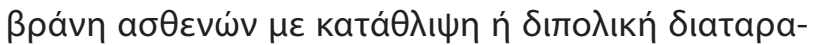

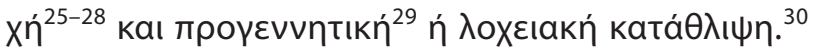

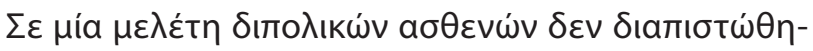

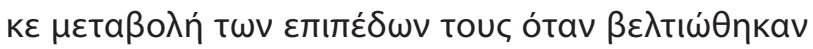

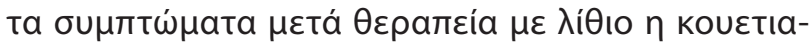




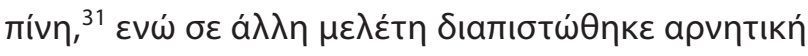

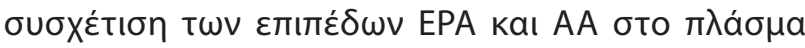

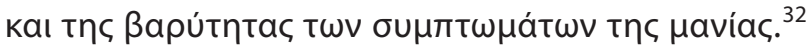

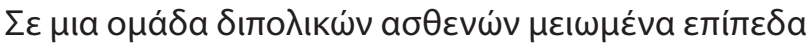

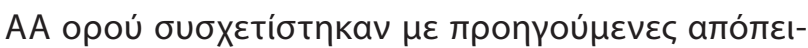

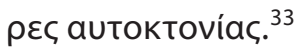

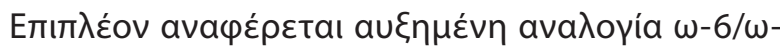

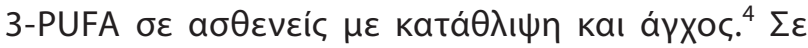

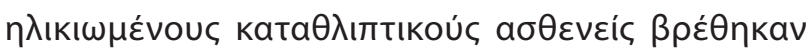

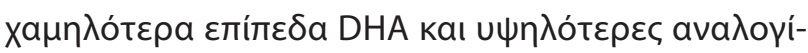

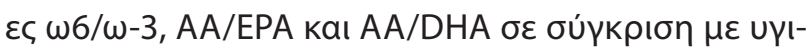

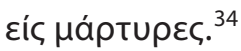

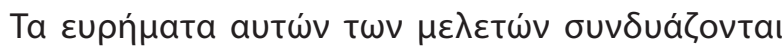

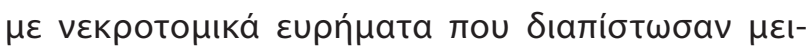

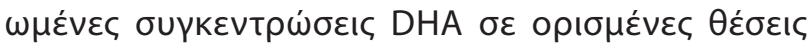

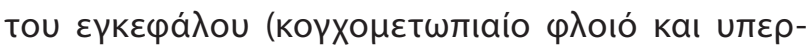

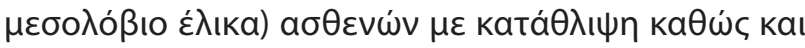

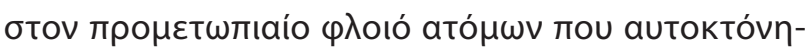

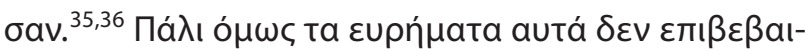
$\dot{\omega} \theta \eta \kappa a v ~ \sigma \varepsilon$ á $\lambda \lambda \varepsilon \varsigma \mu \varepsilon \lambda \varepsilon \dot{\varepsilon} \tau \varsigma \varsigma_{,}^{4,37}$

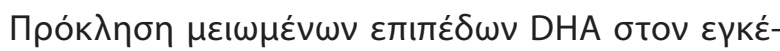

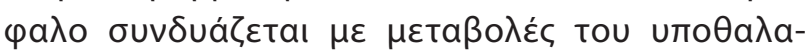

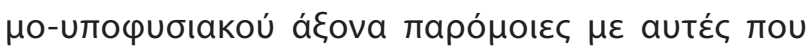

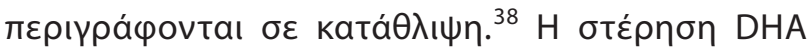

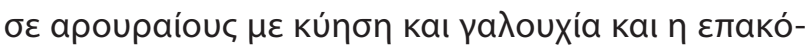

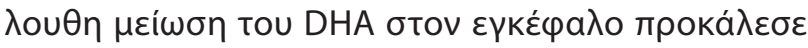

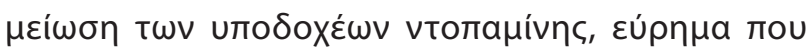

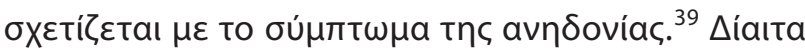

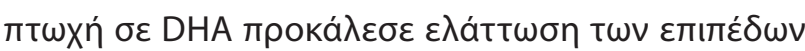

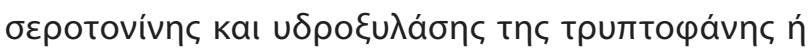

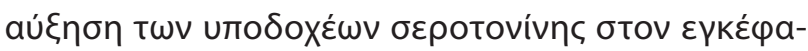
$\lambda$ a apoupaíwv. ${ }^{40,41}$

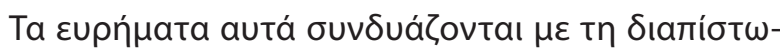

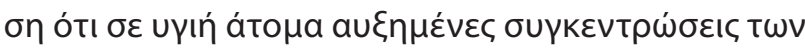

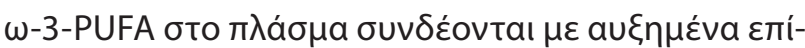

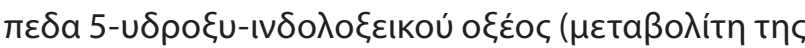

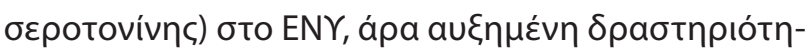
ta oepotovívnc. ${ }^{42}$

\section{y. Хopŕynon PUFA}

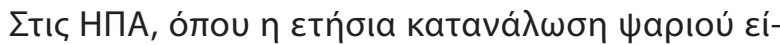

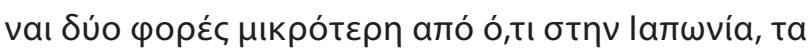

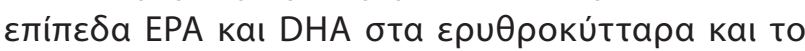

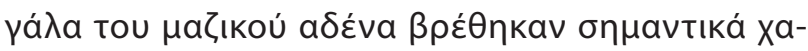

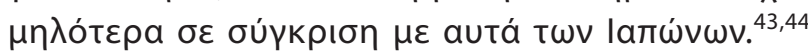

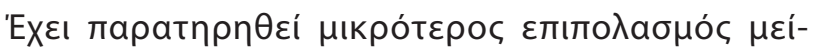

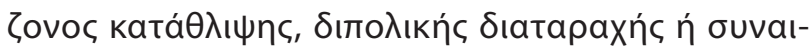

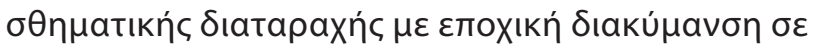

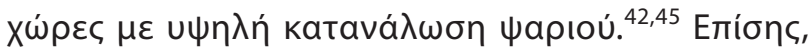

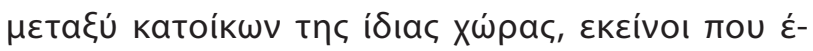

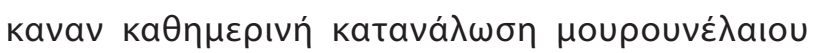

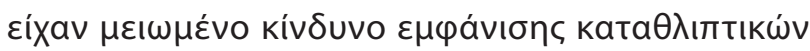

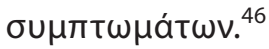

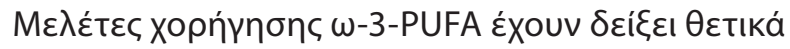

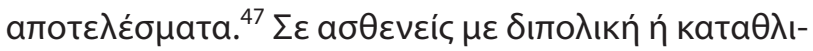

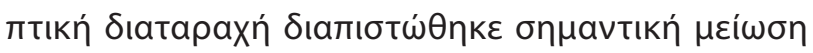

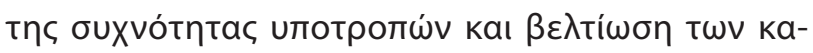

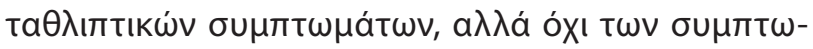

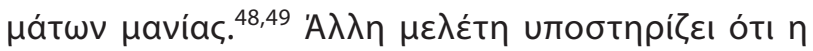

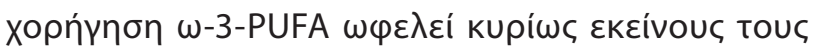

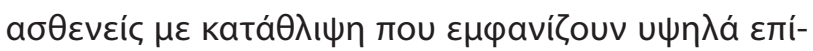

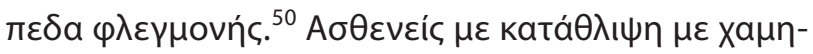

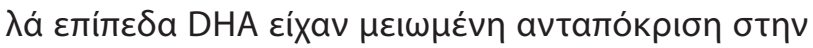

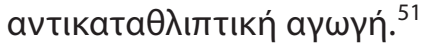

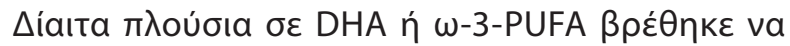

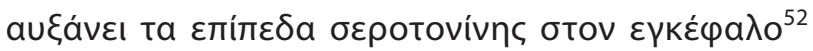

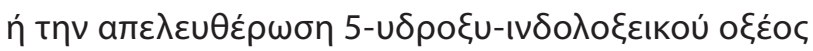

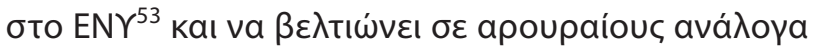

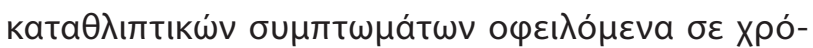

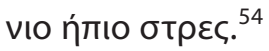

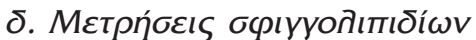

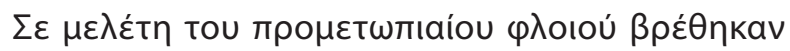

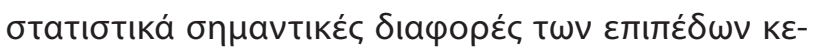

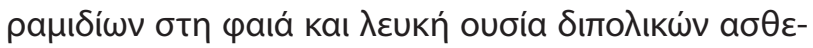

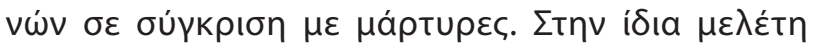

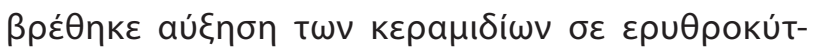

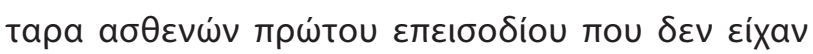

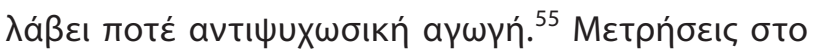

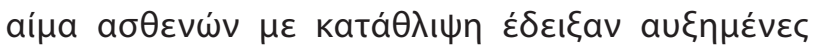

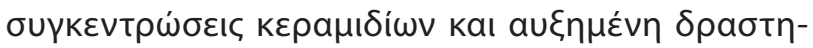

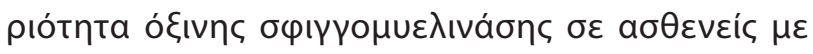

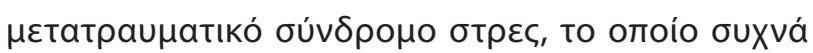

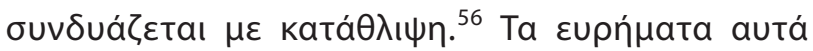

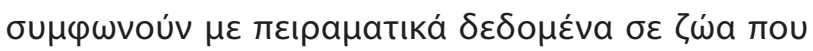

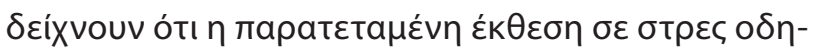

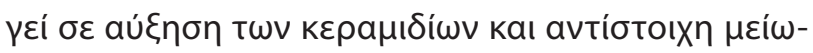

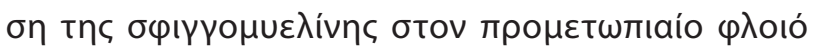
каı тоV Ітпо́канто. ${ }^{57}$ 


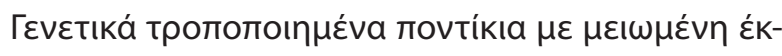

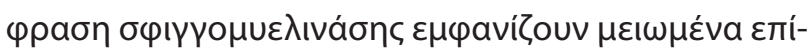

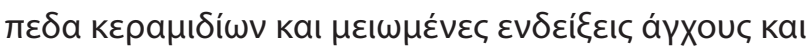

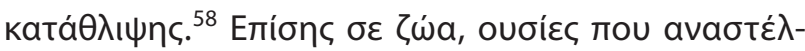

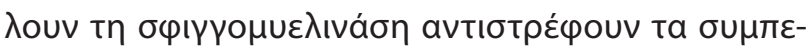

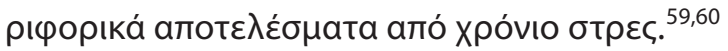

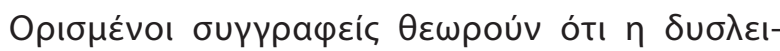

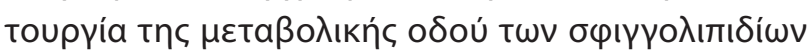

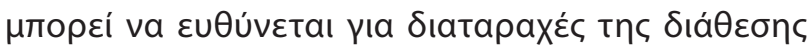

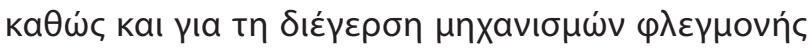

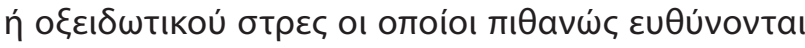

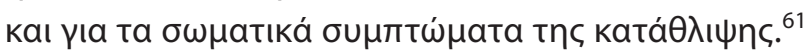

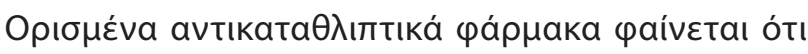

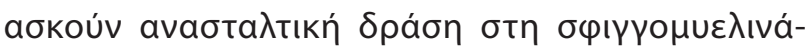

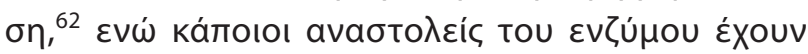

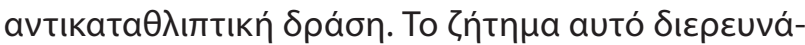

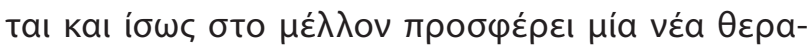

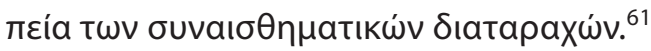

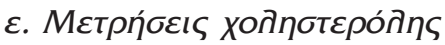

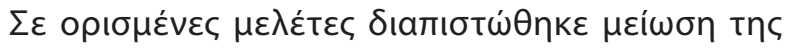

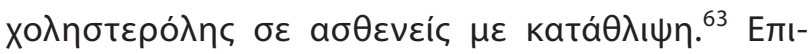

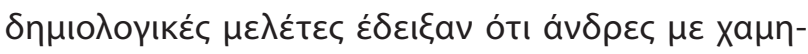

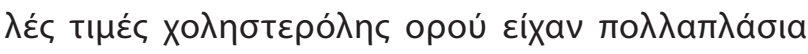

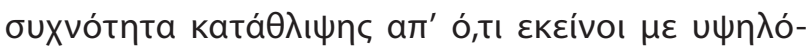

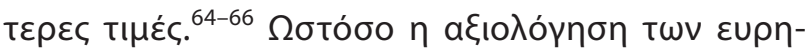

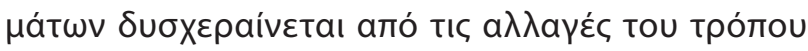

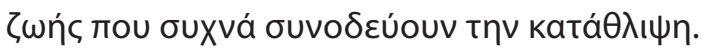

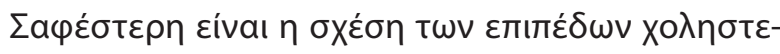

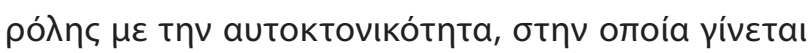

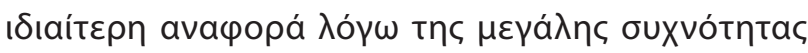

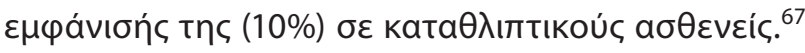

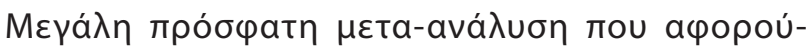

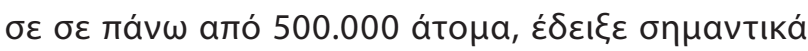

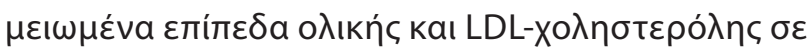

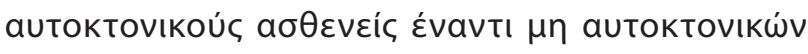

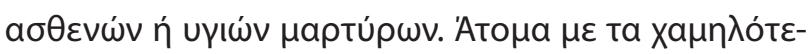

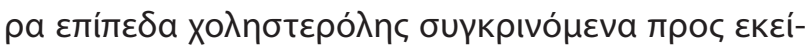

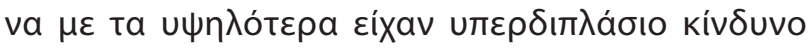

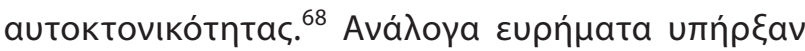

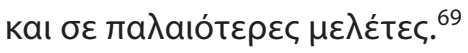

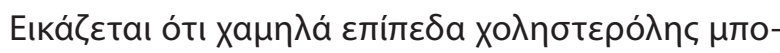

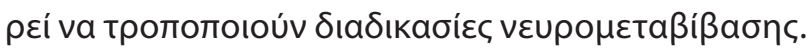

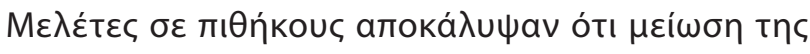

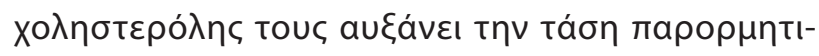

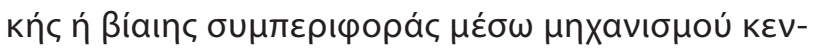

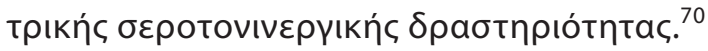

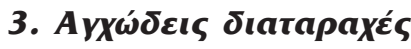

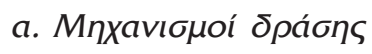

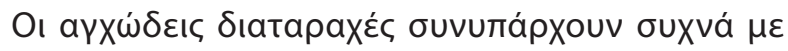

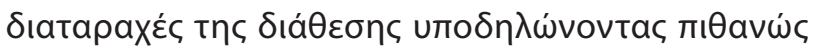

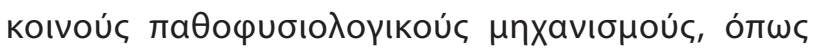
$\delta$

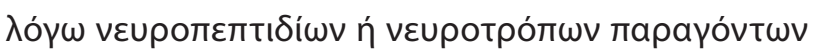

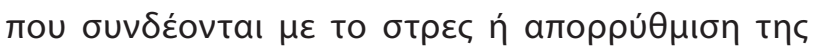

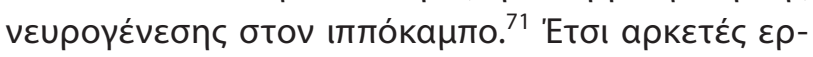

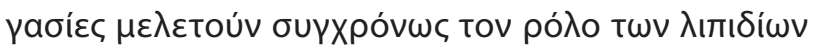

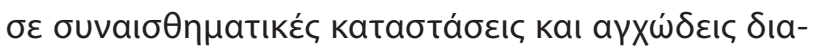
Tapax $\varsigma^{67}{ }^{67}$

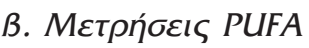

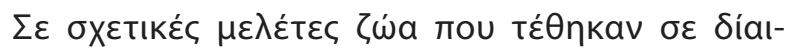

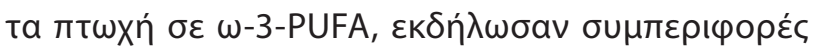

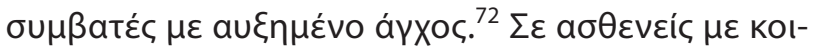

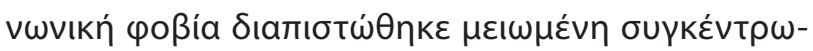

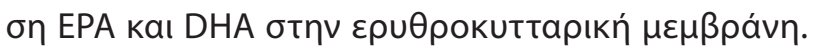

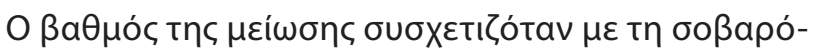

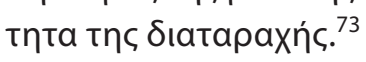

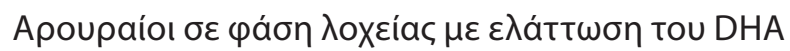

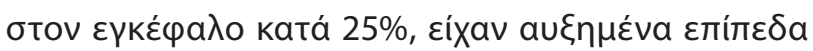

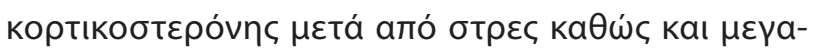

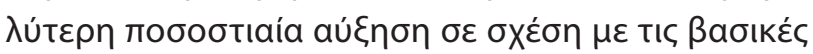

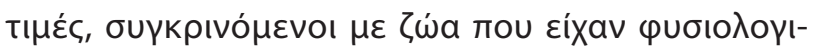

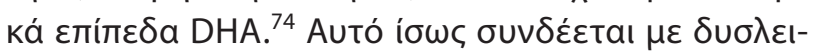

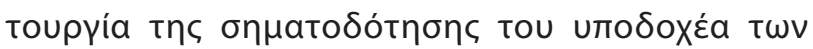

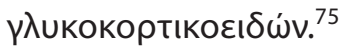

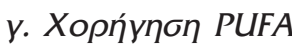

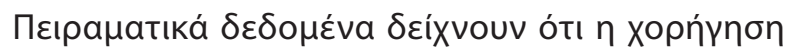

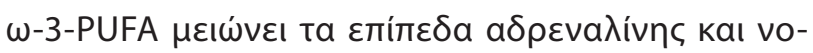

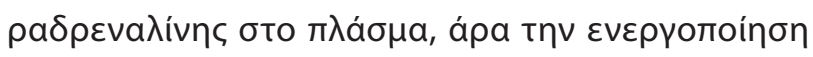

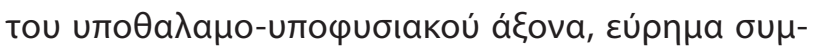

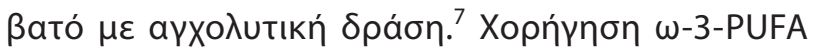

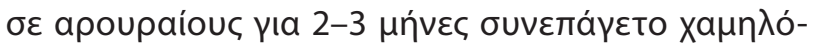

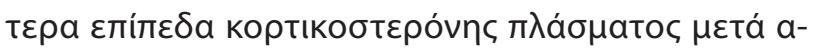

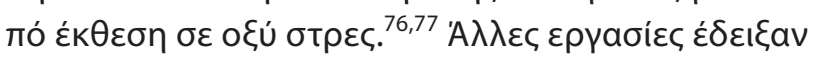

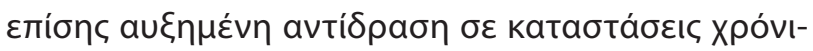

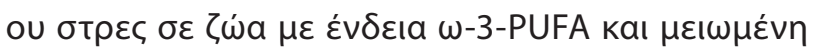

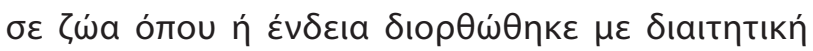




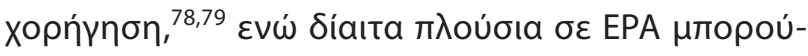

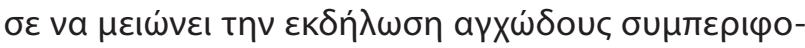
pác. ${ }^{80}$

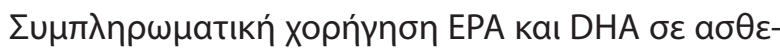

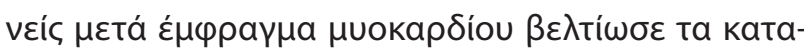

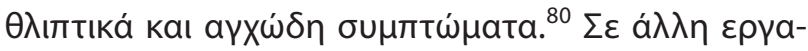

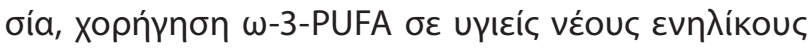

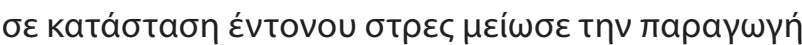

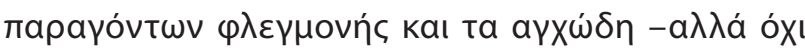
та катаӨ

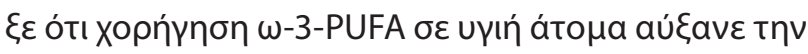

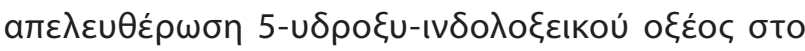

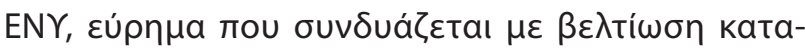

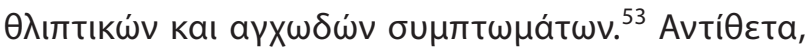

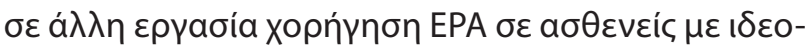

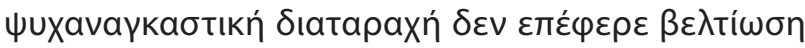

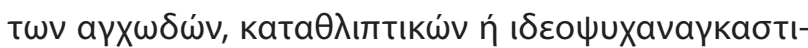

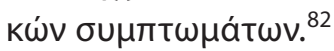

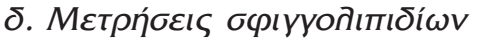

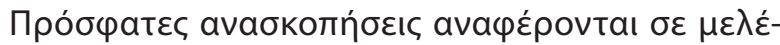

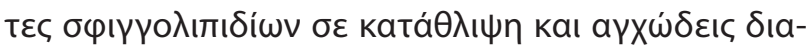

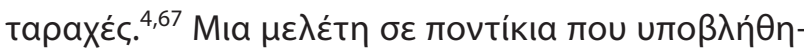

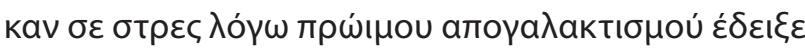

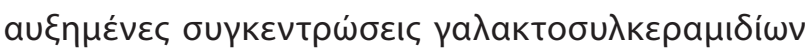

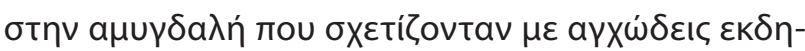
$\lambda \omega \dot{\sigma \varepsilon ા \zeta . ~}{ }^{83}$

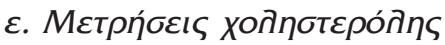

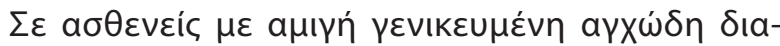

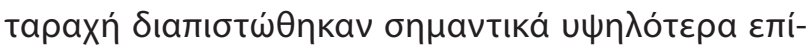

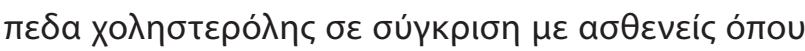

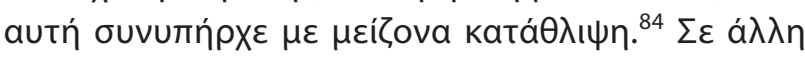

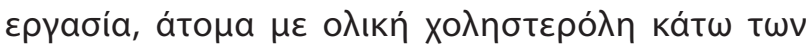

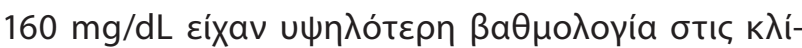

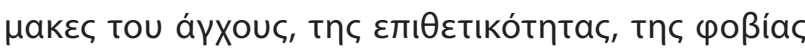

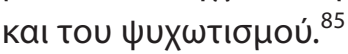

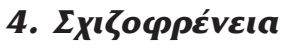

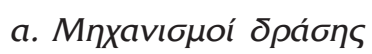

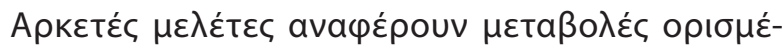

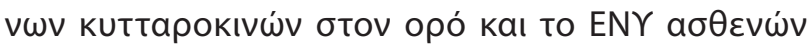

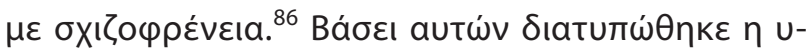

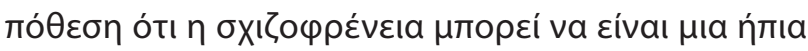

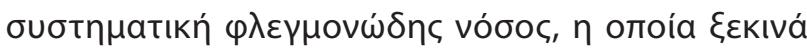

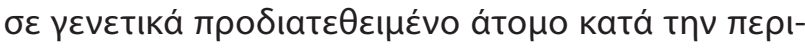

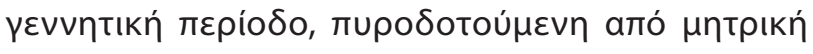

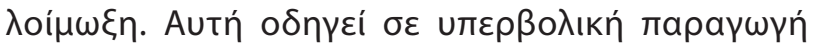

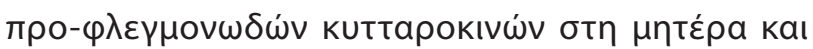

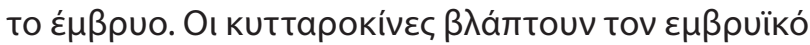

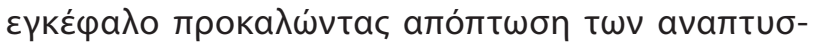

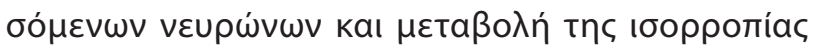

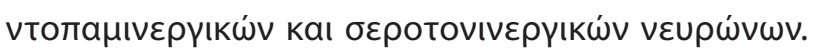

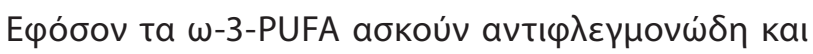

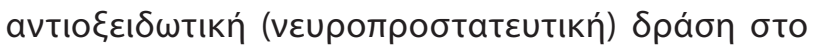

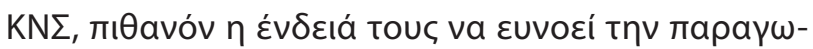

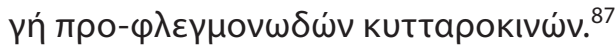

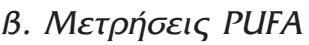

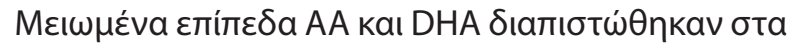

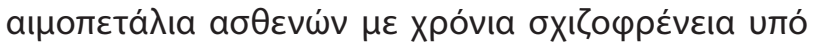

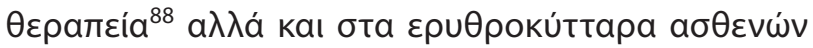

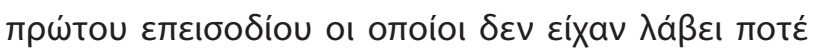

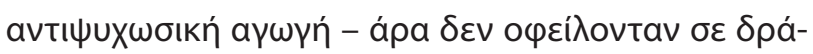

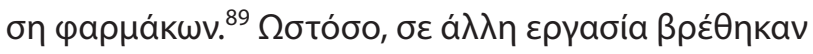

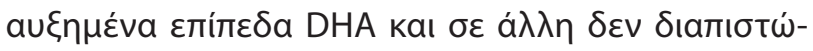

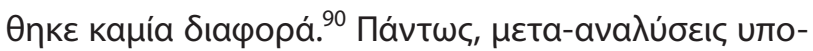

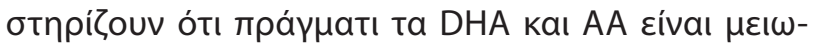

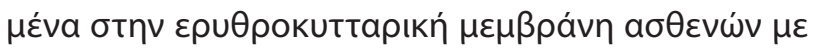

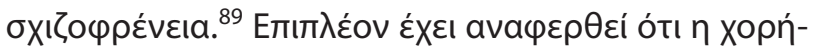

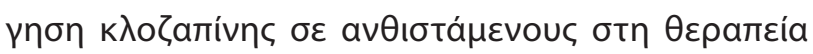

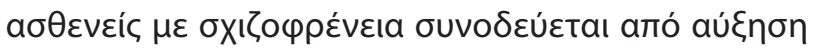

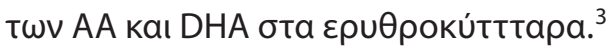

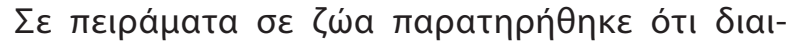

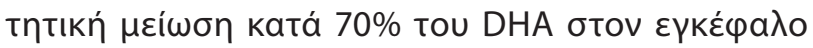

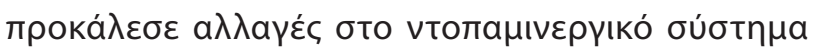

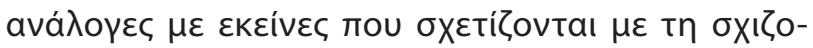

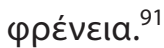

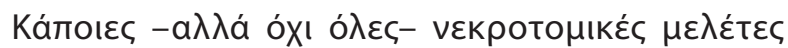

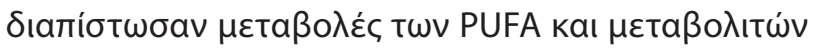

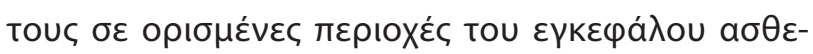

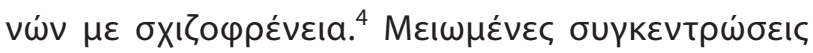

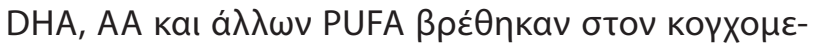

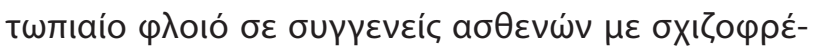
vela. $^{35}$

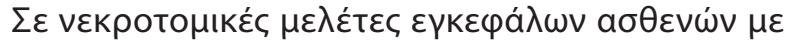

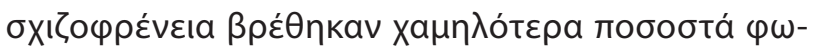

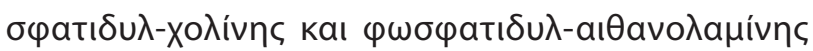

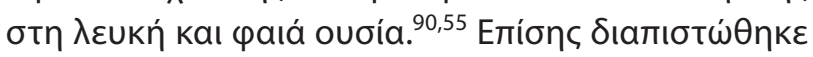

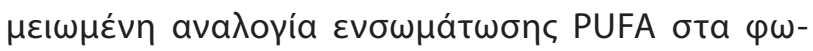

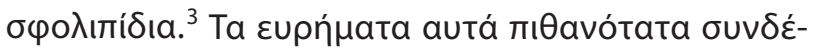




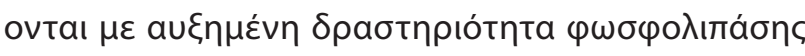

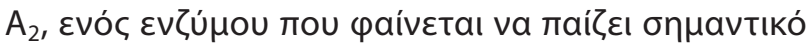

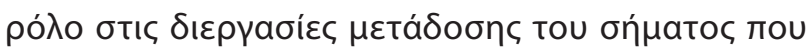

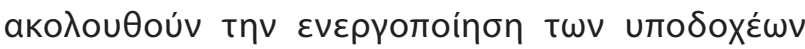

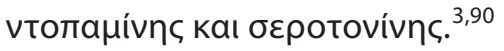

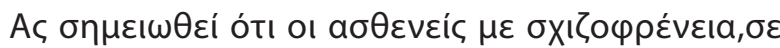

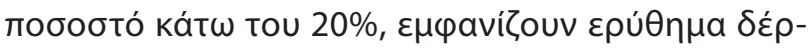

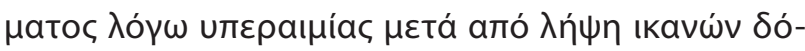

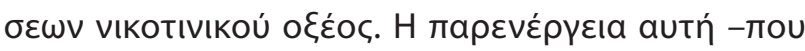

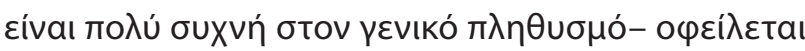

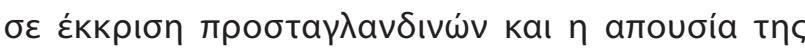

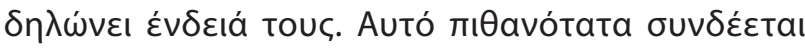

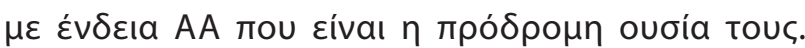

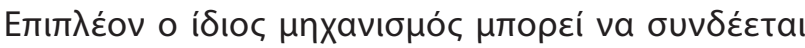

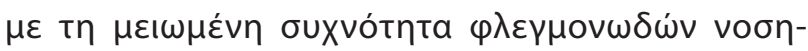

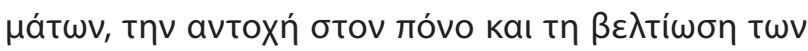

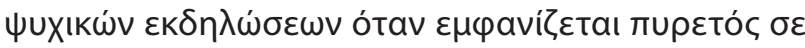

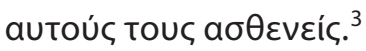

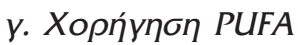

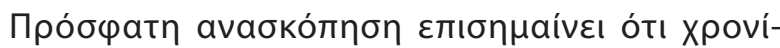

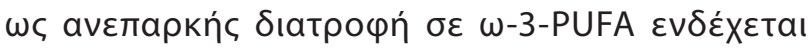

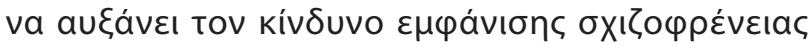

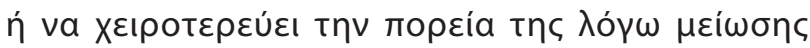

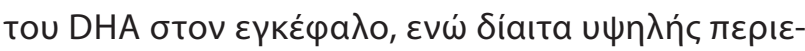

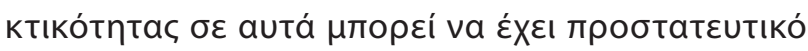

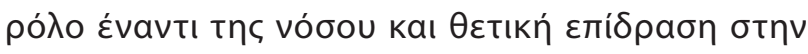
порвía tnc. ${ }^{4}$

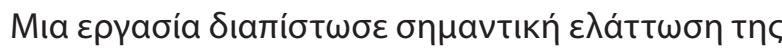

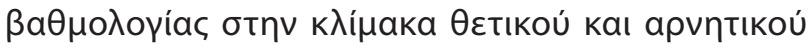

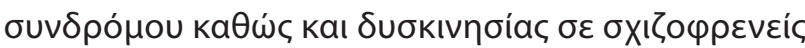

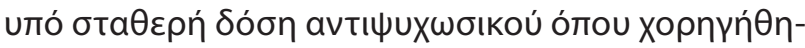

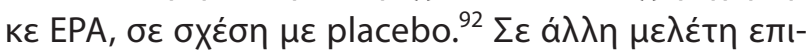

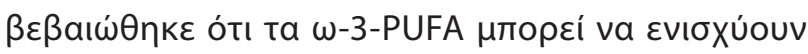

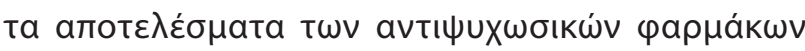

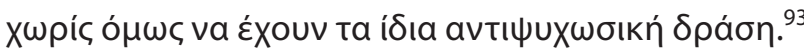

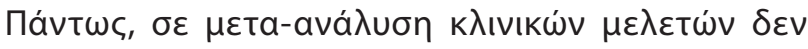

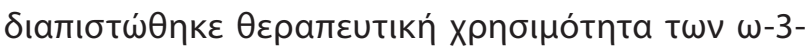

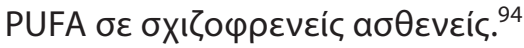

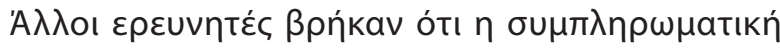

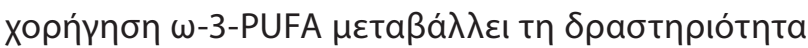

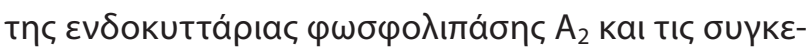

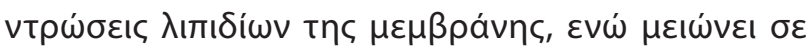

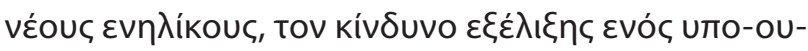

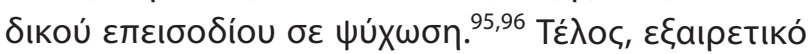

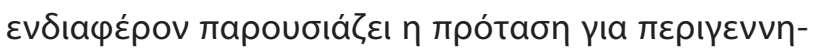

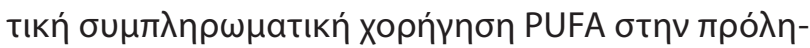

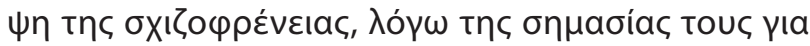

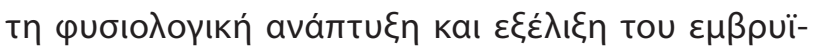

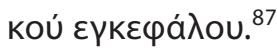

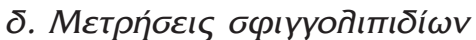

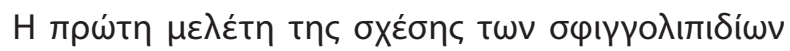

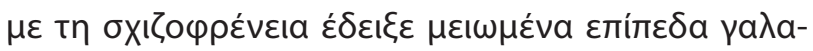

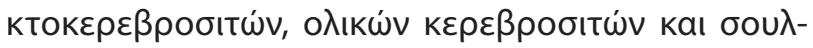

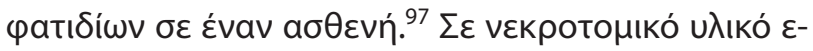

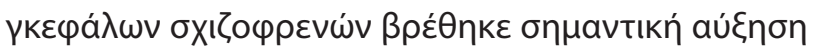

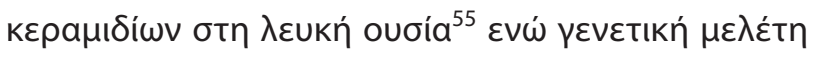

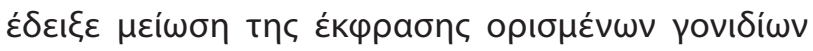

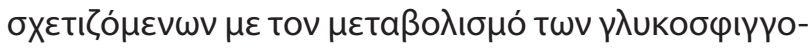
$\lambda \iota \pi เ \delta i \omega v .{ }^{98}$

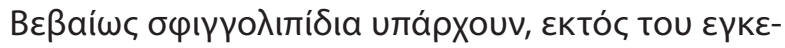

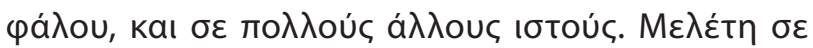

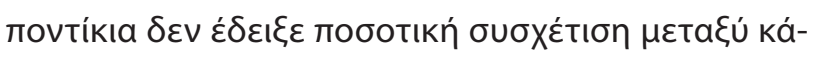

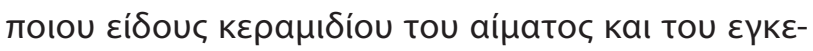

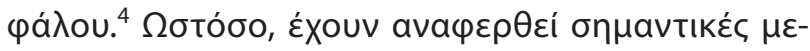

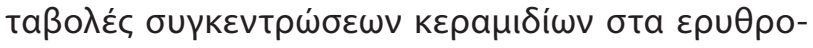

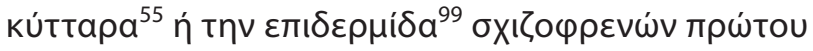

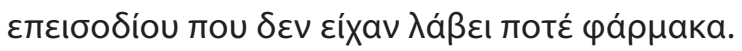

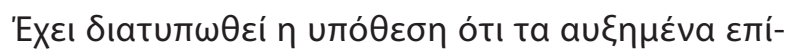

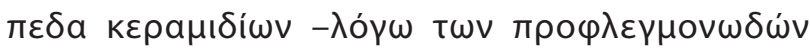

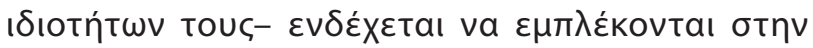

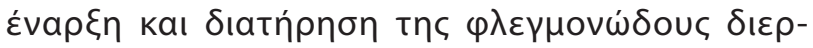

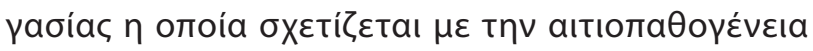

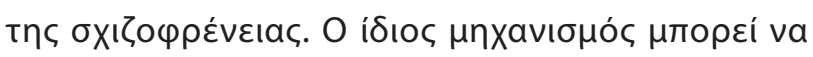

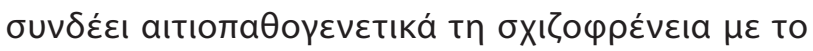

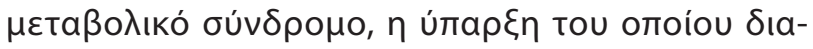

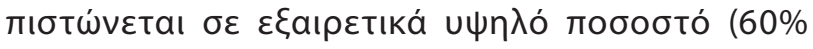

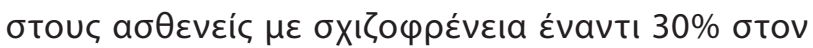
үعVIKó $\pi \lambda \eta \theta \cup \sigma \mu o ́) .{ }^{100}$

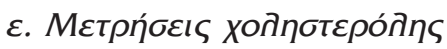

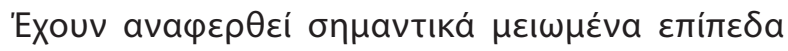

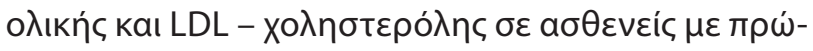

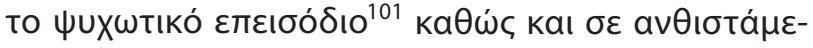

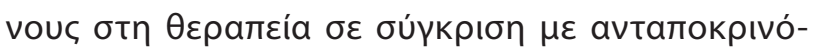

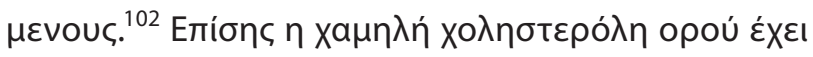

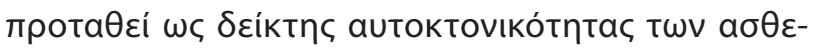

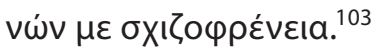




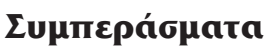

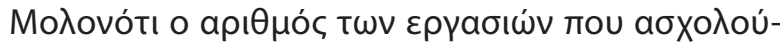

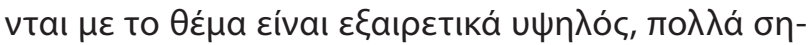

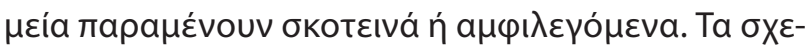

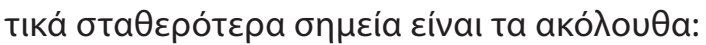

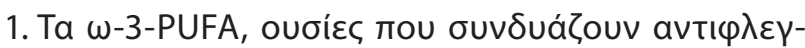

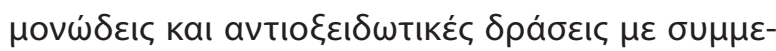

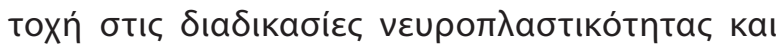

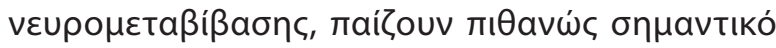

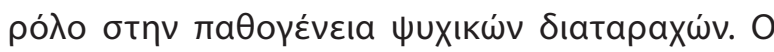

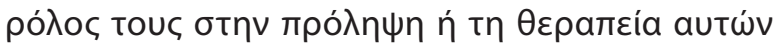

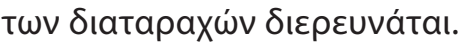

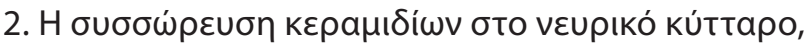

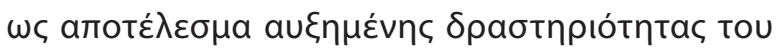

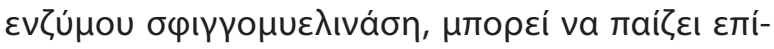

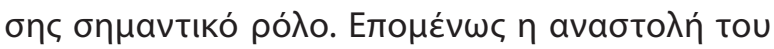

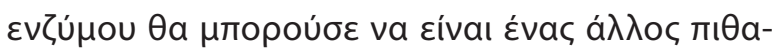

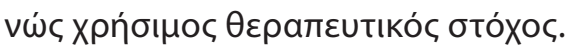

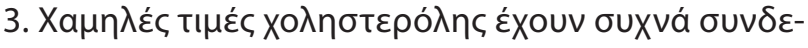

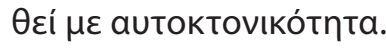

\title{
Lipids and mental disorders: Evidence, uncertainties and perspectives
}

\author{
St. Theodoropoulou, ${ }^{1}$ A.G. Gialouris ${ }^{2}$ \\ ${ }^{1}$ Psychiatric Department, General Hospital "Evaggelismos", Athens, \\ ${ }^{2}$ Department of Internal Medicine, General Hospital "Elpis", Athens, Greece
}

Psychiatriki 2019, 30:129-141

Brain is an organ with the highest lipid concentration in the body. Cellular membrane lipids can affect both the positioning and the functioning of membrane proteins, thus regulating several cell actions. Changes in the lipid composition of cell membrane can modulate the microenvironment and consequently the function of its proteins, e.g. neurotransmission. Some of the first studies on the subject have shown a negative correlation between serum cholesterol levels and depression, suicidality and behavioral disorders. Several studies -but not all- have found decreased concentrations of $\omega-3$ polyunsaturated fatty acids ( $\omega$-3-PUFA) in plasma or erythrocyte membranes of patients with depression, bipolar disorder or after a suicide attempt. In some cases, positive results after their administration have been reported. The effect of $\omega$-3-PUFA in affective disorders is attributed to their action on neurotransmission, neuroplasticity as well as to their anti-inflammatory and anti-oxidative properties. Besides, decreased levels of $\omega$-3-PUFA have been found in erythrocytes or platelets of schizophrenic patients. Some studies have shown in schizophrenics an increased rate of membrane phospholipids breakdown and a decreased ratio of $\omega$-3-PUFA incorporation in phospholipids, possibly because of increased activity of phospholipase $A_{2}$, an enzyme with crucial role in signaling transduction. Deficient dietetic ingestion of $\omega$-3-PUFA may increase the risk for development of schizophrenia, while a diet rich in $\omega$-3-PUFA may have a preventive role for the disease or improve its course. Although there is no evidence for their action as a monotherapy, they may be useful as an add-on therapy to drug treatment. Some authors suggest that abnormal sphingolipid metabolism, leading to accumulation of ceramides, may be responsible for the development of mood and anxiety disorders, as well as for induc- 
tion of inflammation or oxidative stress, mechanisms possibly responsible for the physical symptoms of depression. Some drugs seem to combine inhibition of sphingomyelinase (an enzyme catalyzing the production of ceramides) and antidepressant effect. Despite the multitude of related studies, many aspects of the subject remain obscure. Current research focuses on the validity of preventive (especially in the perinatal period) or therapeutic administration of $\omega$-3-PUFA as well as to the pharmacological manipulation of enzymes involved in lipid metabolism (e.g. sphingomyelinase) for the treatment of psychiatric disorders.

Key words: Affective disorders, anxiety disordes, schizophrenia, cholesterol, $\omega-3$ polyunsaturated fatty acids, ceramides.

\section{BıßAtoypacpía}

1. Hallahan B, Garland MR. Essential fatty acids and mental health. $\mathrm{Br} J$ Psychiatry 2005, 186:275-277, doi: 10.1192/ bjp. 186.4.275

2. Bourre JM, Dumont O, Piciotti M, Clement M, Chaudiere J, Bonneil $M$ et al. Essentially of w3 fatty acids for brain structure and function. World Rev Nutr 1991, 66:103-117, PMID: 2053331

3. Horrobin DF. The membrane phospholipid hypothesis as a biochemical basis for the neurodevelopmental concept of schizophrenia. Schizophr Res 1998, 30:193 208, PMID: 9589514

4. Schneider M, Levant B, Reichel M, Gulbin E, Kornhuber J, Müller CP. Lipids in psychiatric disorders and preventive medicine. Neurosci Biobehav Rev 2017, 76:336-362, doi: 10.1016/j. neubiorev.2016.06.002

5. de la Presa Owens S, Innis SM. Docosahexaenoic and arachidonic acid prevent a decrease in dopaminergic and serotoninergic neurotransmitters in frontal cortex caused by a linoleic and alpha-linolenic acid deficient diet in formula-fed piglets. J Nutr 1999, 129:2088-2093, doi:1093/in/129.11.2088

6. Kodas E, Galineau L, Bodard S, Vancassel S, Guilloteau D, Besnard JC et al. Serotoninergic neurotransmission is affected by $n-3$ polyunsaturated fatty acids in the rat. $J$ Neurochem 2004, 89:695-702, doi: 10.1111/j.1471-4159.2004.02401.x

7. Hamazaki K, Itomura M, Huan M, Nishizawa H, Sawazaki S, Tanouchi $M$ et al. Effect of omega-3 fatty acid-containing phospholipids on blood catecholamine concentrations in healthy volunteers: a randomized placebo-controlled, double-blind trial. Nutr 2005, 21:705-710, doi: 10.1016/j.nut.2004.07.020

8. Darios F, Davletov B. Omega-3 and omega-6 fatty acids stimulate cell membrane expansion by acting on syntaxin 3. Nature 2006, 440:813-817, doi: 10.1038/nature04598

9. Kawakita E, Hashimoto M, Shido O. Docosahexaenoic acid promotes neurogenesis in vitro and in vivo. Neuroscience 2006,139:991-997, doi: 10.1016/j.neuroscience.2006.01.021

10. Tokuda H, Kontani M, Kawashima H, Kiso Y, Shibata H, Osumi N. Differential effect of arachidonic acid and docosahexaenoic acid on age-related decreases in hippocampal neurogenesis. Neurosci Res 2014, 88:58-66, doi: 1016j.neures,2014.08.002
11. Muldoon MF, Manuck SB, Matthews KA. Lowering cholesterol concentrations and mortality: a quantitative review of primary prevention trials. BMJ 1990, 301:309-314, PMID: 2144195

12. Muldoon MF, Rossouw EJ, Manuck BS, Glueck JC, Kaplan RJ, Kaufmann PG. Low or lowered cholesterol and risk of death from suicide and trauma. Metabolism 1993, 42(Suppl):45-56, doi: 10.1016/0026-0495(93)90259Q

13. Fritze J, Schneider B, Lanczik M, Autoaggressive behaviour and cholesterol. Neuropsychobiology 1992, 26:180-181, doi:10.1159/000118916

14. Kuan-Pin Su, Matsuoka Y, Chi-Un Pae. Omega-3 polyunsaturated fatty acids in prevention of mood and anxiety disorders. Clin Psychopharmacol Neurosci 2015, 13:129-137, doi: 10.9758/cpn.2015.13.2.129

15. Stoll AL, Severus WE. Mood stabilizers shared mechanisms of action at post-synaptioc signal transduction and kindling processes. Harv Rev Psychiatry 1996, 4:77-89, doi: 10.3109/ 10673229609030527

16. Chen G, Manji HK, Hawver DB, Wright CB, Potter WZ. Chronic sodium valporate selectively decreases protein kinase $\mathrm{C}$ a and e in vitro. J Neurochem 1994, 63:2361-2364, PMID: 7964759

17. Blondeau N, Nguemeni C, Debruyne DN, Piens M, Wu X, Pan $\mathrm{H}$ et al. Subchronic alpha-linolenic acid treatment enhances brain plasticity and exerts an antidepressant effect: a versatile potential therapy for stroke. Neuropsychopharmacology 2009, 34:2548-2559, doi: 10.1038/npp.200984

18. Venna VR, Deplanque D, Allet C, Belarbi K, Hamdane M, Bordet R. PUFA induce antidepressant-like effects in parallel to structural and molecular changes in the hippocampus. Psychoneuroendocrinology 2009, 34:199-211, doi: 10.1016/j. psyneuen.2008.08.025

19. Wu A, Ying Z, Gomez-Pinilla F. Dietary omega-3 fatty acids normalize BDNF levels, reduce oxidative damage, and counteract learning disability after traumatic brain injury in rats. J Neurotrauma 2004, 21:1, doi: 10.1089/neu.2004.21.1457

20. Maes M, Meltzer HYM. The serotonin hypothesis of major depression. In: Bloom FE, Kupfer DJ (eds) Psychopharmaco- 
logy, the Fourth Generation of Progress. Raven Press, New York, 1995

21. Su KP. Inflammation in psychopathology of depression: clinical, biological, and therapeutic implications. BioMedicine 2012, 2:68-74, doi: 10.1016/j.biomed.2012.03.002

22. Duman RS, Malberg J, Thome J. Neural plasticity to stress and antidepressant treatment. Biol Psychiatry 1999, 46:1181-1191, PMID: 10560024

23. Santarelli L, Saxe M, Gross C, Surget A, Battaglia F, Dulawa $S$ et al. Requirement of hippocampal neurogenesis for the behavioral effects of antidepressants. Science 2003, 301:805809, doi: $10.1126 /$ science. 1083328

24. Beltz BS, Tlusty MF, Benton JL, Sandeman DC. Omega-3 fatty acids upregulate adult neurogenesis. Neurosci Lett 2007, 415:154-158, doi: 10.1016/j.neulet 2007.01.010

25. Maes A, Delanghe J, Altamura C, Neels H, Meltzer HY. Lowered $\omega 3$ polyunsaturated fatty acids in serum phospholipids and cholesteryl esters of depressed patients. Psychiatry Res 1999, 85:275-291, doi: 10.1016/S0165-1781(99)00014-1

26. McNamara RK, Jandacek R, Rider T, Tso P, Dwivedi $Y$, Pandey GN. Selective deficits in erythrocyte docosahexaenoic acid composition in adult patients with bipolar disorder and major depressive disorder. J Affect Disord 2010, 126:303-311, doi: 10.1016/j.jad.2010.03.015

27. McNamara RK, Welge JA. Meta-analysis of erythrocyte polyunsaturated fatty acid biostatus in bipolar disorder. Bipolar Disord 2016, 18:300-306, doi: 10.1111/bdi.12386

28. Ranjekar PK, Hinge A, Hegde MV, Ghate M, Kale A, Sitasawad $S$ et al. Decreased antioxidant enzymes and membrane essential polyunsaturated fatty acids in schizophrenic and bipolar mood disorder patients. Psychiatry Res 2003, 121:109-122, PMID: 14656446

29. Chang JP, Lin CY, LinPY, Shih YH, Chiu TH, Ho M et al. Polyunsaturated fatty acids and inflammatory markers in major depressive episodes during pregnancy. Prog Neuro Psychopharmacology Biol Psychiatry 2018, 80:273-278, doi: 10.1016/j.pnpbp.2017.05.008

30. de Vriese SR, Christophe AB, Maes M. Lowered serum n-3 polyunsaturated fatty acid (PUFA) levels predict the occurrence of postpartum depression: further evidence that lowered n-PUFAs are related to major depression. Life Sci 2003,73:3181-3187, PMID: 14561523

31. McNamara RK, Jandacek R, Tso P, Blom TJ, Welge JA, Strawn $\mathrm{JR}$ et al. First-episode bipolar disorder is associated with erythrocyte membrane docosahexaenoic acid deficits: Dissociation from clinical response to lithium or quetiapine. Psychiatry Res 2015, 230:447-453, doi: 10.1016/j.psychres.2015.09.035

32. Sublette ME, Bosetti F, DeMar JC, Ma K, Bell JM, Fagin-Jones $S$ et al. Plasma free polyunsaturated fatty acid levels are associated with symptom severity in acute mania. Bipolar Disord 2007, 9:759-765, doi: 10.1111/j.1399-5618.2007.00387.x

33. Evans SJ, Prossin AR, Harrington GJ, Kamali M, Ellingrod VL, Burant CF et al. Fats and factors: lipid profiles associate with personality factors and suicidal history in bipolar subjects. PLoS One 2012, 7, doi: 10.1371/journal.pone.0029297

34. Tiemeier H, van Tuijl HR, Hofman A, Kiliaan AJ, Breteler MM. Plasma fatty acid composition and depression are associated in the elderly: the Rotterdam Study. Am J Clin Nutr 2003, 78:40-46, doi: $10.1093 /$ ajcn/78.1.40

35. McNamara RK, Hahn CG, Jandacek R, Rider T, Tso P, Stanford KE et al. Selective deficits in the omega-3 fatty acid docosahexaenoic acid in the postmortem orbitofrontal cortex of patients with major depressive disorder. Biol Psychiatry 2007, 62:17-24, doi: 10.1016/j.biopsych.2006.08.026

36. McNamara RK, Jandacek R, Tso P, Dwivedi Y, Ren X, Pandey GN. Lower docosahexaenoic acid concentrations in the postmortem prefrontal cortex of adult depressed suicide victims compared with controls without cardiovascular disease. $J$ Psychiatry Res 2013, 47:1187-1191, doi: 10.1016/j.jpsychires. 2013.05.007

37. Beskow J, Gottfries CG, Roos BE, Winblad B. Determination of monoamine and monoamine metabolites in the human brain: post mortem studies in a group of suicides and in a control group. Acta Psychiatr Scand 1976, 53:7-20, doi: 10.1111/j.1600-0447.1976.tb00054.x

38. Plotsky PM, Owens MJ, Nemeroff CB. Psychoneuroendocrinology of depression. Hypothalamic-pituitary-adrenal axis. Psychiatr Clin N Am 1998, 21:293-307, doi: 10.1016/S0193-953X(05) 70006-X

39. Davis PF, Ozias MK, Carlson SE, Reed GA, Winter MK, McCarson KE et al. Dopamine receptor alterations in female rats with diet-induced decreased brain docosahexaenoic acid (DHA): interactions with status. Nur Neurosci 2010, 13:161169, doi: $10.1179 / 147683010 \times 12611460764282$

40. McNamara RK, Able J, Liu Y, Jandacek R, Rider T, Tso P et al. Omega-3 fatty acid deficiency during perinatal development increases serotonin turnover in the prefrontal cortex and decreases midbrain tryptophan hydroxylase-2 expression in adult female rats: dissociation from estrogenic effects. J Psychiatr Res 2009, 43:656-663, doi: 10.1016/j. jpsychires.2008. 09.011

41. McNamara RK, Jandacek R, Rider T, Tso P, Cole-Strauss A, Lipton JW. Omega-3 fatty acid deficiency increases constitutive pro-inflammatory cytokine production in rats: relationship with central serotonin turnover. Prostaglandins Leukot Essent Fatty Acids 2010, 83: 185-191, doi: 10.1016/j.plefa.2010.08.004

42. Hibbeln JR. Fish consumption and major depression. Lancet 1998, 351:1213, doi: 10.1016/S0140-6736(05)79168-6

43. WHO. World Health Organization Fish and Fishery Products. World Apparent Consumption Based On Food Balance Sheets (1961-1993). Food and Agriculture Organization. Rome: 1996, FAO Fisheries Circular, 821 Rev 3

44. Brenna JT, Varamini B, Jensen RG, Diersen-Schade DA, Boettcher JA, Arterburn LM. Docosahexaenoic and arachidonic acid concentrations in human breast milk worldwide. Am J Clin Nutr 2007, 85:1457-1464, doi: 10.1093/ajcn/85.6.1457 
45. Hibbeln JR, Nieminen LR, Blasbalg TL, Riggs JA, Lands WE. Healthy intakes of $n-3$ and $n-6$ fatty acids: estimations considering worldwide diversity. Am J Clin Nutr 2006, 83:1483S-1493S, doi: 10.1093/ajcn/83.6.1483S

46. Raeder MB, Steen VM, Vollset SE, Bjelland I. Associations between cod liver oil use and symptoms of depression: the Hordaland Health Study. J Affect Disord 2007, 101:245-249, doi: 10.1016/j.jad.2006.11.006

47. Messamore E, Almeida DM, Jandacek RJ, McNamara RK. Polyunsaturated fatty acids and recurrent mood disorders: Phenomenology, mechanisms, and clinical application. Prog Lipid Res 2017, 66:1-13, doi: 10.1016/j.plipres.2017.01.001

48. Stoll AL, Severus WE, Freeman MP, Rueter S, Zboyan HA, Diamond $\mathrm{E}$ et al. Omega 3 fatty acids in bipolar disorder: a preliminary double-blind, placebo-controlled trial. Arch Gen Psychiatry 1999, 56:407-412, doi: 10.1001/archpsyc.56.5.407

49. Yang JR, Han D, Qiao ZX, Tian X, Qi D, Qiu XH. Combined application of eicosapentaenoic acid and docosahexaenoic acid on depression in women: a meta-analysis of double-blind randomized controlled trials. Neuropsychiatr Dis Treat 2015, 11:2055-2061, doi: 10.2147/NDT.S86581

50. Rapaport MH, Nierenberg AA, Schettler PJ, Kinkead B, Cardoos A, Walker $R$ et al. Inflammation as a predictive biomarker for response to omega-3 fatty acids in major depressive disorder: a proof-of-concept study. Mol Psychiatry 2016, 21:71-79, doi: $10.1038 / \mathrm{mp} .2015 .22$

51. Mocking RJ, Verburg HF, Westerink AM, Assies J, Vaz FM, Koeter MW et al. Fatty acid metabolism and its longitudinal relationship with the hypothalamic-pituitary-adrenal axis in major depression: associations with prospective antidepressant response. Psychoneuroendocrinology 2015, 59:1-13, doi: 10.1016/j.psyneuen.2015.04.027

52. Carabelli B, Delattre AM, Pudell C, Mori MA, Suchecki D, Machado RB et al. The antidepressant-like effect of fish oil:possible role of ventral hippocampal 5-HT post-synaptic receptor. Mol Neurobiol 2014, 52:206-215, doi: 10.1007/ s12035-014-8849-8

53. Nizzo MC, Tegos S, Gallamini A, Toffano G, Polleri A, Massarotti M. Brain cortex phospholipids liposomes effects on CSF HVA, 5-HIAA and on prolactin and somatotropin secreTranstion in man. J Neural Transm 1978, 43:93-102, PMID: 731234

54. Tang M, Jiang P, Li H, Liu Y, Cai H, Dang R et al. Fish oil supplementation alleviates depressant-like behaviors and modulates lipid profiles in rats exposed to chronic unpredictable mild stress. BMC Complement Altern Med 2015, 1:239, doi: 10.1186/s12906-015-0778-1

55. Schwarz E, Prabakaran S, Whitfield P, Major H, Leweke FM, Koethe $D$ et al. High throughput lipidomic profiling of schizophrenia and bipolar disorder brain tissue reveals alterations of free fatty acids, phosphatidylcholines, and ceramides. $J$ Proteome Res 2008, 10:4266-4277, doi: 10.1021/pr800188y

56. Hammad SM, Truman JP, Al Gadban MM, Smith KJ, Twal WO, Hamner MB. Altered blood sphingolipidomics and elevated plasma inflammatory cytokines in combat veterans with posttraumatic stress disorder. Neurobiol Lipids 2012, 10:2

57. Oliveira TG, Chan RB, Bravo FV, Miranda A, Zhou B, Marques $\mathrm{F}$ et al. The impact of chronic stress on the rat brain lipidome. Mol Psychiatry 2016, 21:80-88, doi: 10.1038/mp.2015.14

58. Gulbins E, Palmada M, Reichel M, Lüth A, Böhmer C, Amato $D$ et al. Acid sphingomyelinase/ceramidesystem mediates effects of antidepressant drugs. Nat Med 2013, 19:934-938, doi: $10.1038 / \mathrm{nm} .3214$

59. Kornhuber J, Tripal P, Reichel M, Mühle C, Rhein C, Mühlbacher $M$ et al. Functional inhibitors of acid sphingomyelinase (FIASMAs): a novel pharmacological group of drugs with broad clinical applications. Cell Physiol Biochem 2010, 26:9-20, doi: 10.1159/000315101

60. Kornhuber J, Muehlbacher M, Trapp S, Pechmann S, Friedl $A$, Reichel $M$ et al. Identification of novel functional inhibitors of acid sphingomyelinase. J Affect Disord 2017, 15:213:35-43, doi: 10.1016/j.jad.2017.02.008

61. Kornhuber J, Müller CP, Becker KA, Reichel M, Gulbins E. The ceramide system as a novel antidepressant target. Trends Pharmacol Sci 2014, 35:293-304, doi: 10.1016/j.tips. 204.04.003

62. Kornhuber J, Reichel M, Tripal P, Groemer TW, Henkel AW, Mühle $C$ et al. The role of ceramide in major depressive disorder. Eur Arch Psychiatry Clin Neurosci 2009, 2:199-204, doi: 10.1007/s00406-009-0061-x

63. Maes R, Smith A, Christophe E, Vandoolaeghe A, Van Gastel $\mathrm{V}$, Neels $\mathrm{H}$ et al. Lower serum high-density lipoprotein cholesterol (HDL-C) in major depression and in depressed men with serious suicidal attempts: relationship with immune-inflammatory markers. Acta Psychiatr Scand 1997, 95:212-221, doi: 10.1111/j.1600-0447.1997.tb09622.x

64. van Reedt Dortland AK, Giltay EJ, van Veen T, van Pelt J, Zitman FG, Penninx BW. Associations between serum lipids and major depressive disorder: results from the Netherlands Study of Depression and Anxiety (NESDA). J Clin Psychiatry 2010, 71:729-736, doi: 10.4088/JCP.08m04865blu

65. Morgan RE, Palinkas LA, Barrett-Connor EL, Wingard DL. Plasma cholesterol and depressive symptoms in older men. Lancet 1993, 341:75-79, PMID: 8093404

66. Steegmans PHA, Hoes AW, Bak AAA, van der Does E, Grobbee $D E$. Higher prevalence of depressive symptoms in middleaged men with low serum cholesterol levels. Psychosom Med 2000, 62:205-211 205, PMID: 10772398

67. Müller CP, Reichel M, Mühle C, Rhein C, Gulbins E, Kornhuber J. Molecular and cell biology of lipids brain membrane lipids in major depression and anxiety disorders. Biochim Biophys Acta 2015, 1851:1052-1065, doi: 10.1016/j.bbalip.2014.12. 014

68. Shunquan Wu, Yingying Ding, Fuquan Wu, Guoming Xie, Jun Hou, Panyong Mao. Serum lipid levels and suicidality: a metaanalysis of 65 epidemiological studies. J Psychiatry Neurosci 2016, 41:56-69, doi: 10.1503/jpn.150079 
69. Sullivan PF, Joyce PR, Bulik CM, Mulder RT, Oakley-Browne M. Total cholesterol and suicidality in depression. Biol Psychiatry 1994, 7:472-477, doi: 10.1016/0006-3223(94)90643-2

70. Kaplan JR, Muldoon MF, Manuck SB, Mann JJ. Assessing the observed relationship between low cholesterol and violencerelated mortality. Implications for suicide risk. Ann N Acad Sci 1997, 836:57-80, PMID: 9616794

71. Ross BM. Omega-3 polyunsaturated fatty acids and anxiety disorders. Prostaglandins, Leukot Essent Fatty Acids 2009, 81:309-312, doi: 10.1016/j.plefa.2009.10.004

72. Pifferi F, Dorieux O, Castellano CA, Croteau E, Masson M, Guillermier $M$ et al. Long-chaind n-3 PUFAs form fish oil enhance resting state brain glucoseutilization and reduce anxiety in an adult nonhuman primate, the grey mouselemur. J Lipid Res 2015, 56:1511-1518, doi: 10.1194/jlr.M058933

73. Green P, Hermesh H, Monselise A, Marom S, Presburger G, Weizman A. Red cell membrane omega-3 fatty acids are decreased in non-depressed patients with social anxiety disorder. Eur Neuropsychopharmacol 2006, 16:107113, doi: 10.1016/j.euroneuro.2005.07.005

74. Levant B, Ozias MK, Davis PF, Winter M, Russell KL, Carlson SE et al. Decreased brain docosahexaenoic acid content produces neurobiological effects associated with depression: interactions with reproductive status in female rats. Psychoneuroendocrinology 2008, 33:1279-1292, doi: 10.1016/j.psyneuen.2008.06.012

75. Larrieu T, Hilal ML, Fourrier C, De Smedt-Peyrusse V, Sans N, Capuron $L$ et al. Nutritional omega-3 modulates neuronal morphology in the prefrontal cortex along with depression-related behaviour through corticosterone secretion. Trans/ Psychiatry 2014, 4:e437, doi: 10.1038/tp.2014.77

76. Ferraz AC, Delattre AM, Almendra RG, Sonagli M, Borges C, Araujoet al. Chronic omega-3 fatty acids supplementation promotes beneficial effects on anxiety, cognitive and depressive-like behaviors in rats subjected to a restraint stress protocol. Behav Brain Res 2011, 219:116-122, doi: 10.1016/j. bbr.2010.12.028

77. Jiang L, Liang QY, Shi Y. Pure docosahexaenoic acid can improve depression behaviors and affect HPA axis in mice. Eur Rev Med Pharmacol Sci 2012, 16:1765-1773, PMID: 23208960

78. Harauma A, MoriguchiT. Dietary $n-3$ fatty acid deficiency in mice enhances anxiety induced by chronic mild stress. Lipids 2011, 46:409-416, doi: 10.1007/s11745-010-3523-z

79. Hennebelle M, Balasse L, Latour A, Champeil-Potokar G, Denis $\mathrm{S}$, Lavialle $\mathrm{M}$ et al. Influence of omega-3 fatty acid status on the way rats adapt to chronic restraint stress. PLoS One 2012, 7:e42142, doi: 10.1371/journal.pone.0042142

80. Su KP, Matsuoka Y, Pae CU. Omega-3 polyunsaturated fatty acids in prevention of mood and anxiety disorders. Clin Psychopharmaco Neurosci 2015, 13:129-137, doi: 10.9758/ cpn.2015.13.2.129

81. Kiecolt-Glaser JK, Belury MA, Andridge R, Malarkey WB, Glaser R. Omega-3 supplementation lowers inflammation and anxiety in medical students: a randomized controlled trial. Brain Behav Immun 2011, 25:1725-1734, doi: 10.1016/j. bbi.2011.07.229

82. Fux M, Benjamin J, Nemets B. A placebo-controlled crossover trial of adjunctive EPA in OCD. J Psychiatr Res 2004, 38:323-325, doi: 10.1016/S0022-3956(03)00077-3

83. Ono M, Kikusui T, Sasaki N, Ichikawa M, Mori Y, MurakamiMurofushi K. Early weaning induces anxiety and precocious myelination in the anterior part of the basolateral amygdala of male Balb/c mice. Neuroscience 2008, 156, 1103-1110, doi: 10.1016/j.neuroscience.2008.07.078.

84. Kuczmierczyk AR, Barbee JG, Bologna NA, Townsend $\mathrm{MH}$. Serum cholesterol levels in patients with generalized anxiety disorder (GAD) and with GAD and comorbid major depression. Can J Psychiatry 1996, 41:465-8, doi: 10.1177/070674379604100712

85. Chwen Cheng Chen, Feng-Hwa Lu, Jin-Shang Wu, Chih-Jen Chang. Correlation between serum lipid concentrations and psychological distress. Psychiatry Res 2001, 102:153-162, PMID: 11408054

86. Theodoropoulou S, Spanakos G, Baxevanis CN, Economou M, Gritzapis AD, Papamichail MP et al. Cytokine serum levels, autologous mixed lymphocyte reaction and surface marker analysis in never medicated and chronically medicated schizophrenic patients. Schizophr Res 2001 47:13-25, PMID: 11163541

87. Das UN. Can perinatal supplementation of long-chain polyunsaturated fatty acids prevent hypertension in adult life? Med Sci Monit 2004, 10:33-37, PMID: 15567990

88. Peet M, Laugharne JD, Mellor J, Ramchand CN. Essential fatty acid deficiency in erythrocyte membranes from chronic schizophrenic patients, and the clinical effects of dietary supplementation. Prostaglandins Leukot Essent Fatty Acids 1996, 55:71-75, PMID: 8888126

89. Hoen WP, Lijmer JG, Duran M, Wanders RJ, van Beveren NJ, de Haan L. Red blood cell polyunsaturated fatty acids measured in red blood cells and schizophrenia: a meta-analysis. Psychiatry Res 2013, 207:1-12, doi: 10.1016/j.psychres. 2012.09.041

90. Du Bois TM, Deng C, Huang XF. Membrane phospholipid composition, alterations in neurotransmitter systems and schizophrenia. Prog NeuroPsychopharmacol Biol Psychiatry 2005, 29:878-888, doi: 10.1016/j.pnpbp.2

91. Chalon, S. Omega-3 fatty acids and monoamine neurotransmission. Prostaglandins Leukot Essent Fatty Acids 2006, 75:259269, doi: 10.1016/j.plefa.2006.07.005

92. Emsley R, Myburgh C, Oosthuizen P, van Rensburg SJ. Randomized, placebo-controlled study of ethyl-eicosapentaenoic acid as supplemental treatment in schizophrenia. Am J Psychiatry 2002, 159:1596-1598, doi: 10.1176/appi. ajp.159.9.1596

93. Emsley R, Chiliza B, Asmal L du Plessis S, Phahladira L, van Niekerk $\mathrm{E}$ et al. A randomized, controlled trial of omega-3 fatty acids plus an antioxidant for relapse prevention after antipsychotic discontinuation in first-episode schizophrenia. Schizophr Res 2014, 158:230-235, doi: 10.1016/j.schres.2014.06.004 
94. Politi P, Rocchetti M, Emanuele E, Rondanelli M, Barale F. Randomized placebo-controlled trials of omega-3 polyunsaturated fatty acids in psychiatric disorders: a review of the current literature. Curr Drug Discov Technol 2013, 10:245-253, PMID: 21838664

95. Amminger GP, Schafer MR, Papageorgiou K, Klier CM, Cotton SM, Harrigan SM et al. Long-chain omega-3fatty acids for indicated prevention of psychotic disorders: a randomized, placebo-controlled trial. Arch Gen Psychiatry 2010, 67, 146-154, doi: 10.1001/archgenpsychiatry.2009.192

96. Amminger GP, Schafer MR, Schlogelhofer M, Klier CM, McGorry PD. Longer-term outcome in the prevention of psychotic disorders by the Viennaomega-3 study. Nat Commun 2015, 6:7934, doi: 10.1038/ncomms8934

97. Cherayil GD. Estimation of glycolipids in four selected lobes of human brain in neurological diseases. J Neurochem 1969 16:913-920, doi: 10.1111/j.1471-4159.1969.tb08980.x

98. Narayan S, Head SR, Gilmartin TJ, Dean B, Thomas EA. Evidence for Disruption of Sphingolipid Metabolism in Schizophrenia. J Neurosci Res 2009, 87:278-288, doi: 10.1002/j nr.21822

99. Smesny S, Schmelzer CE, Hinder A, Köhler A, Schneider C, Rudzok $M$ et al. Skin ceramide alterations in first-episode schizophrenia indicate abnormal sphingolipid metabolism. Schizophr Bull 2013, 39:933-941, doi: 10.1093/schbul/ sbs058

100. Castillo RI, Rojo LE, Henriquez-Henriquez M, Silva $H$, Maturana A, Villar MJ et al. From molecules to the clinic: Linking schizophrenia and metabolic syndrome through sphingolipids metabolism. Front Neurosci 2016, 10:488, doi: 10.3389/fnins.2016.00488
101. Pillinger T, Beck K, Stubbs B, Howes OD. Cholesterol and triglyceride levels in first-episode psychosis: systematic review and meta-analysis. Br J Psychiatry 2017, 211:339-349, doi: 10.1192/bjp.bp.117.200907

102. Boston PF, Dursun SM, Zafar R, Reveley MA. Serum cholesterol and treatment-resistance in schizophrenia. Biol Psychiatry 1996, 40:542-543, doi: 10.1016/0006-3223(96)00102-3

103. Mensi R, Messaoud A, Mhallah A, Azizi I, Salah WH, Douki W et al. The association between altered lipid profile and suicide attempt among Tunisian patients with schizophrenia. Ann Gen Psychiatry 2016,15:36, doi: 10.1186/s12991-016-0123-1

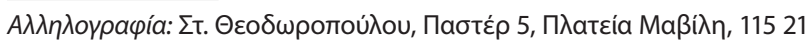

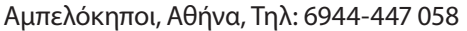
e-mail: st.theodoropoulou@gmail.com 


\title{
Eıठı́кó á $\theta$ $\theta$ o Special article
}

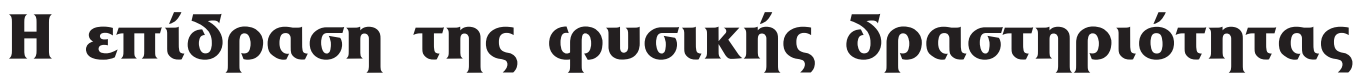 otnv ávoı̀}

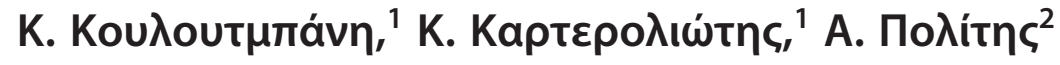

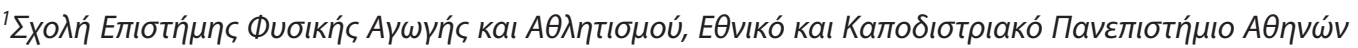

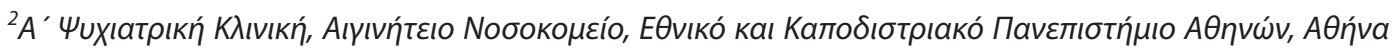

Uuxıатрıкń 2019, 30:142-155

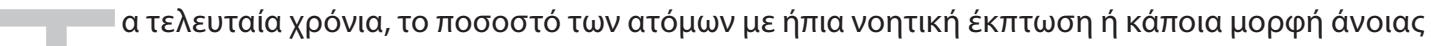

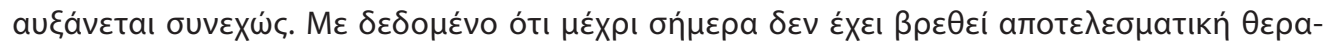

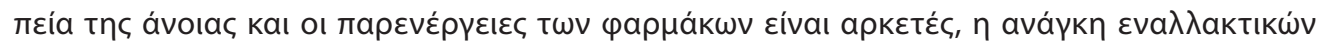

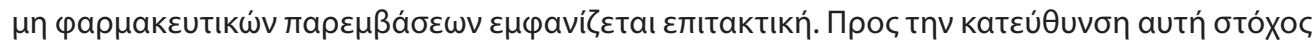

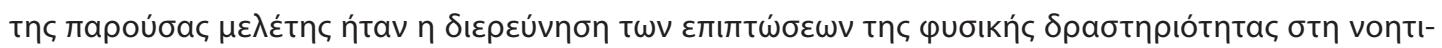

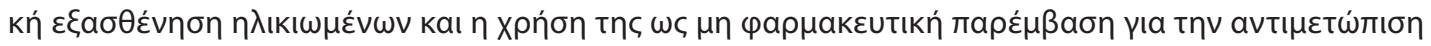

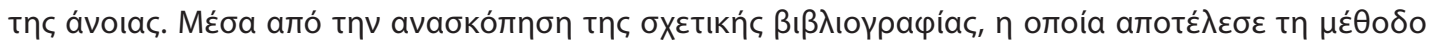

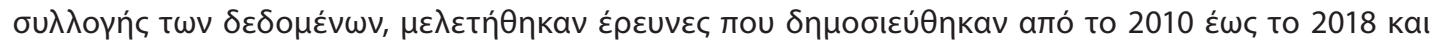

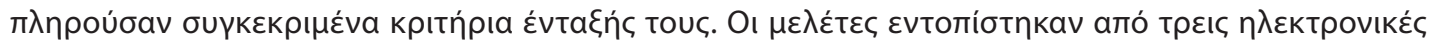

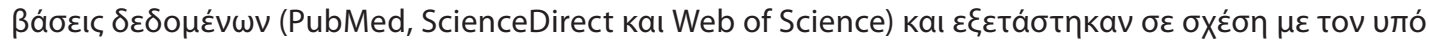

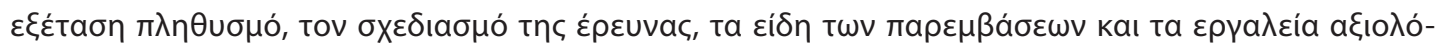

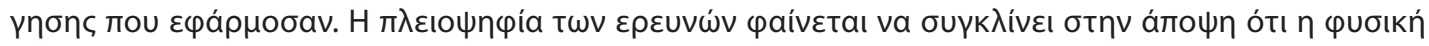

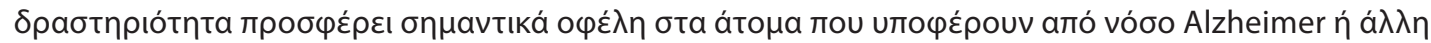

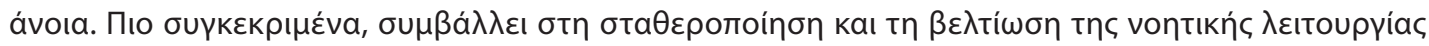

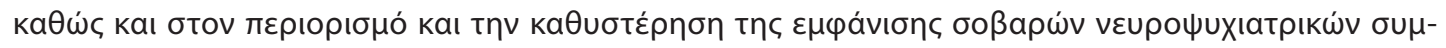

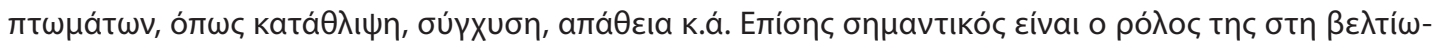

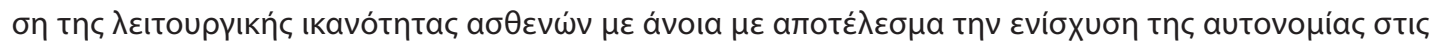

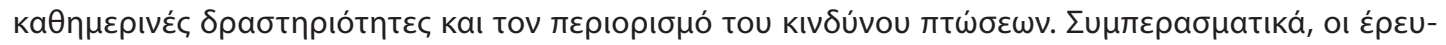

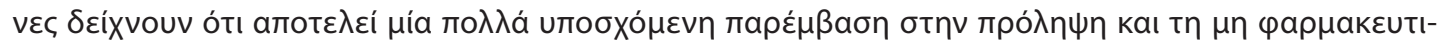

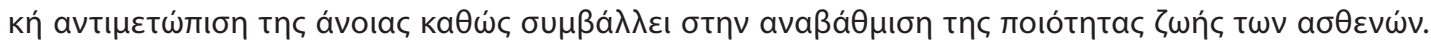

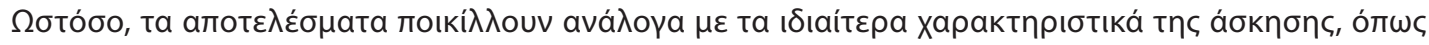

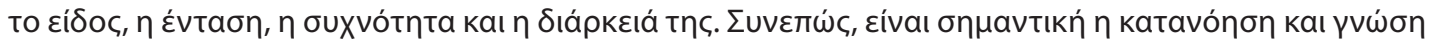




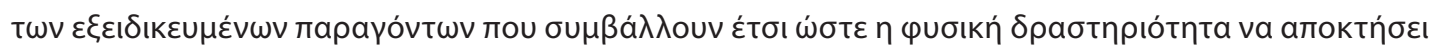

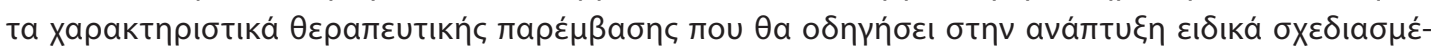

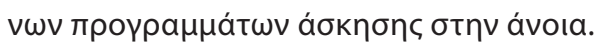

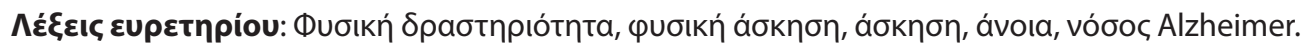

\section{Eıбaywyń}

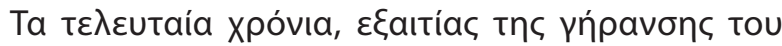

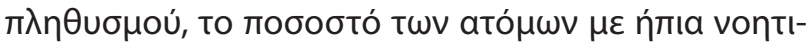

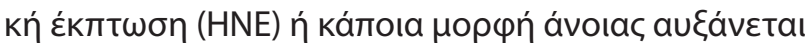

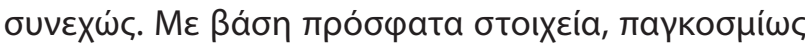

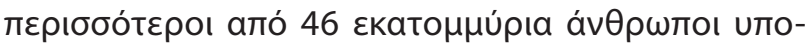

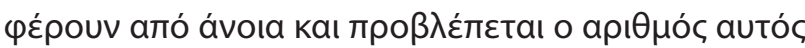

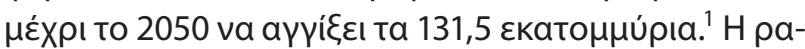

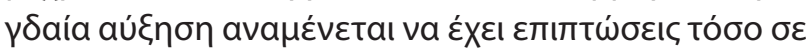

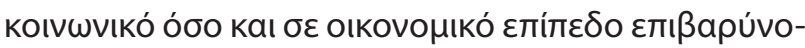

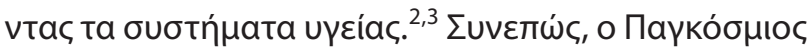

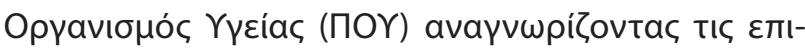

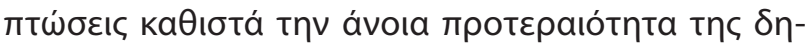

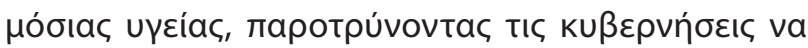

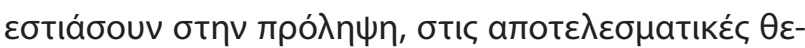

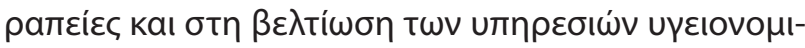

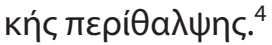

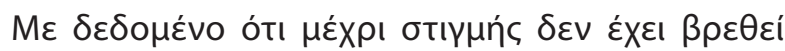

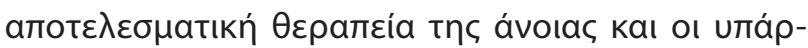

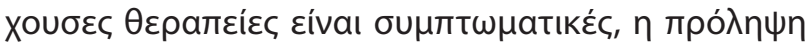

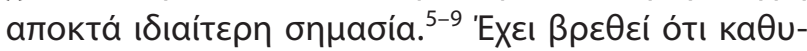

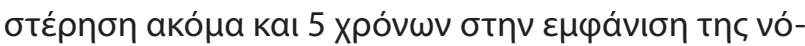

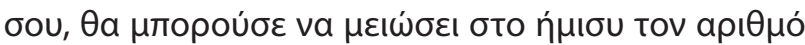

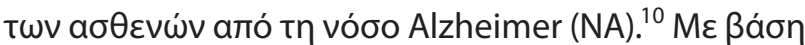

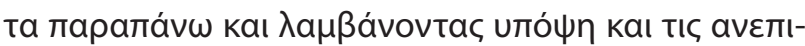

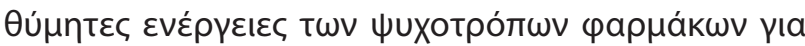

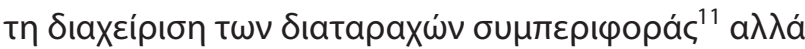

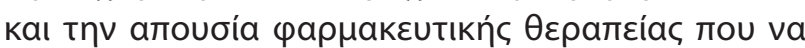

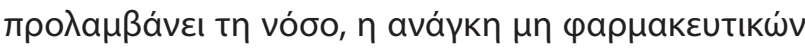

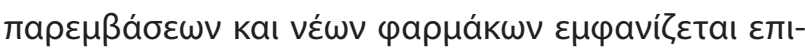
тактікń.

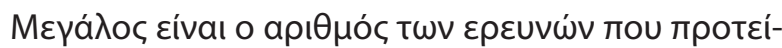

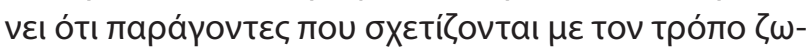

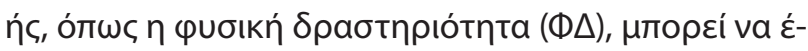

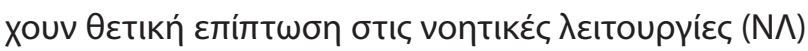

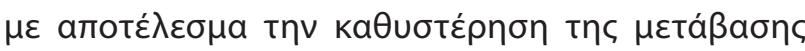

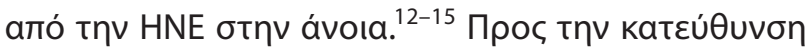

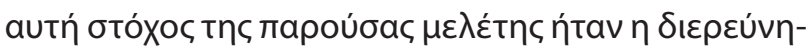

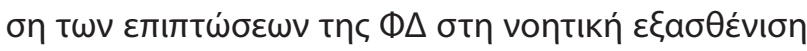

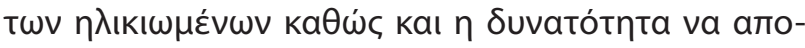

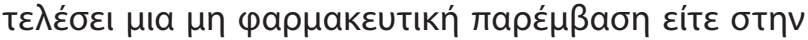

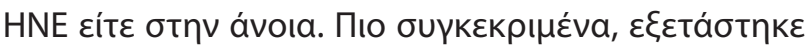

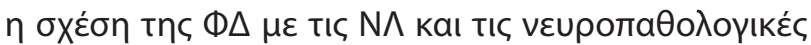

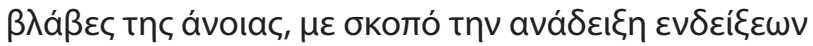

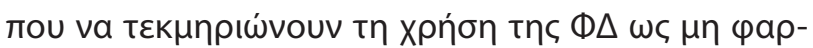

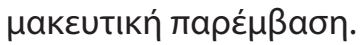

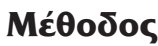

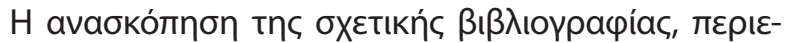

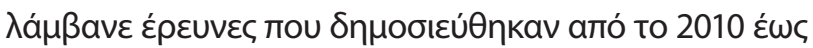

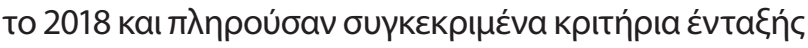

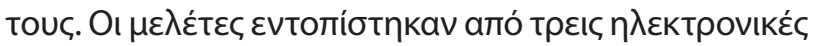

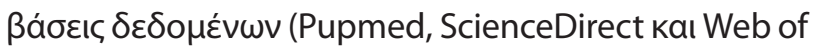

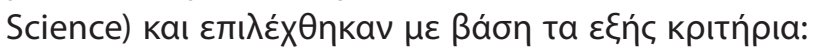

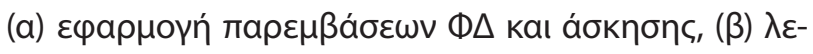

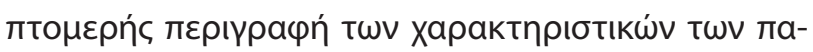

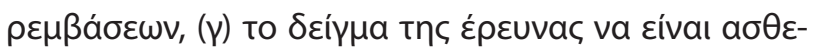

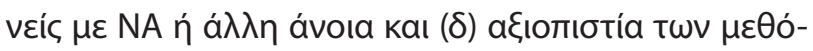

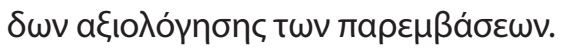

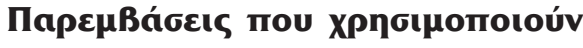

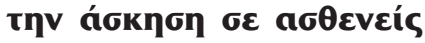

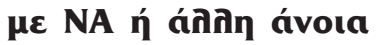

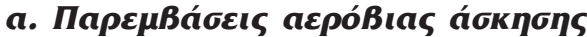

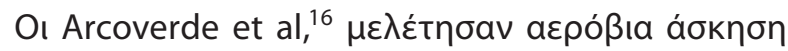

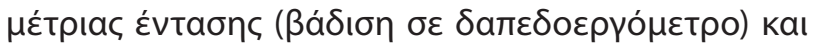

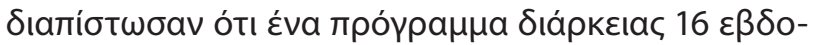

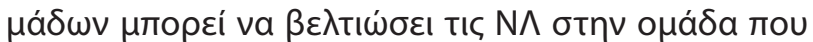

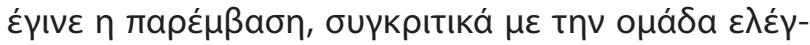

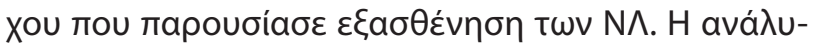

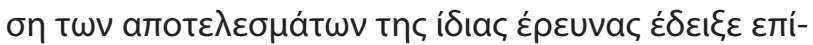

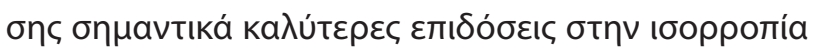

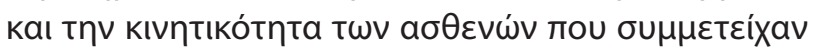

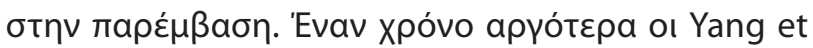

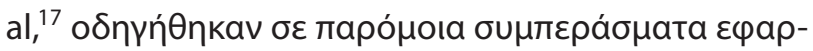

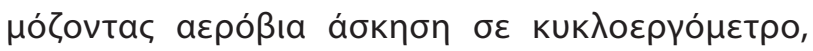

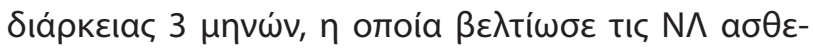

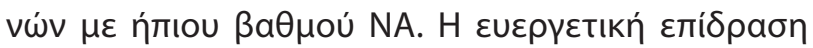

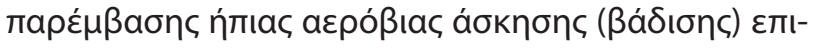

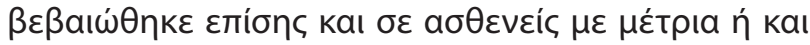




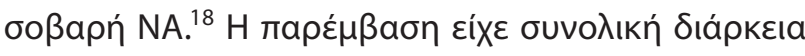

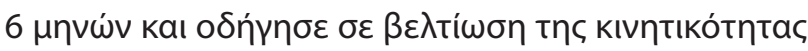

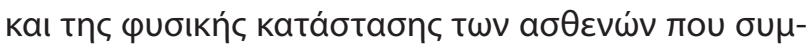

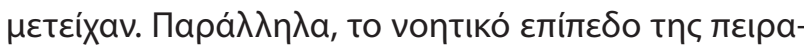

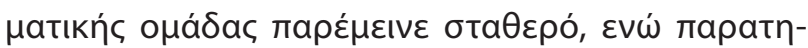

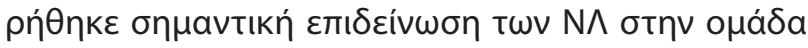

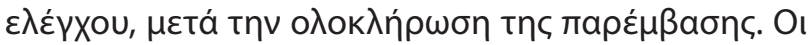

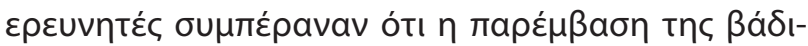

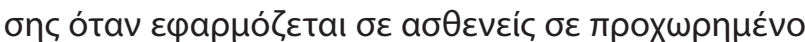

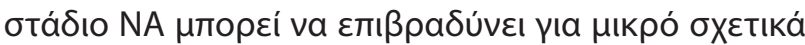

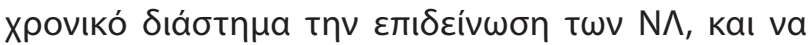

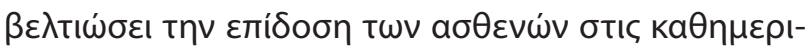

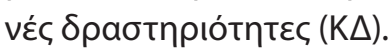

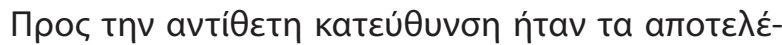

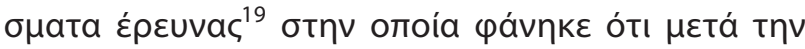

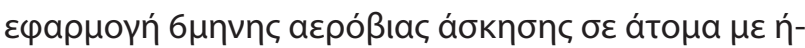

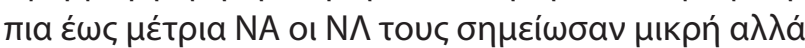

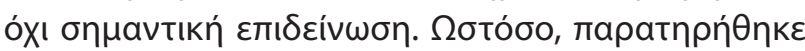

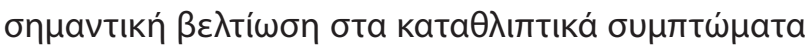

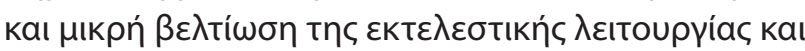

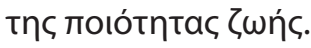

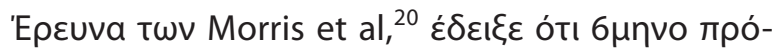

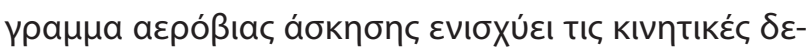

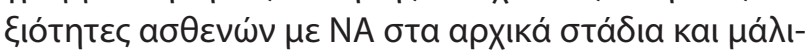

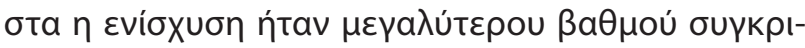

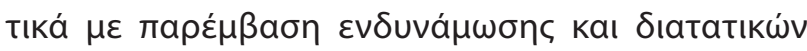

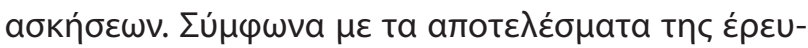

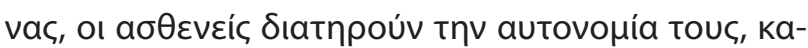

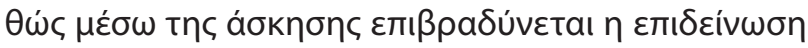

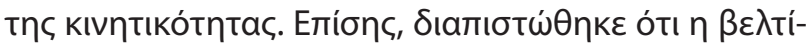

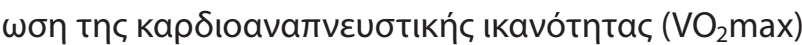

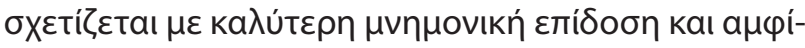

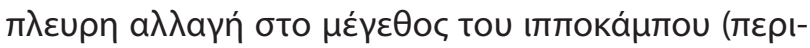

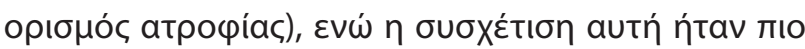

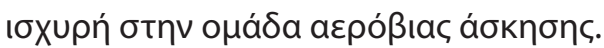

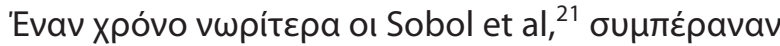

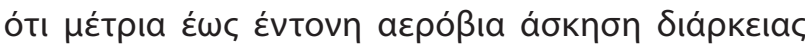

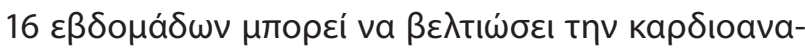

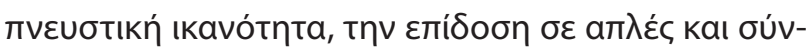

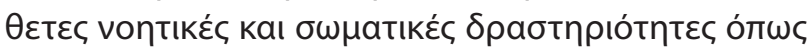

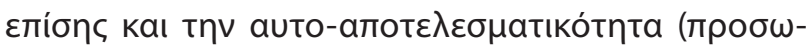

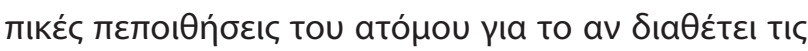

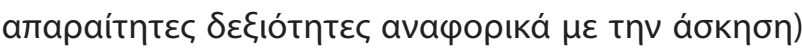

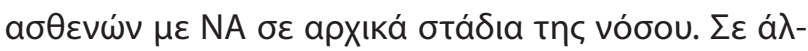

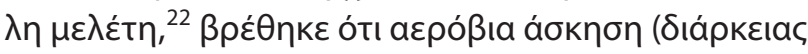

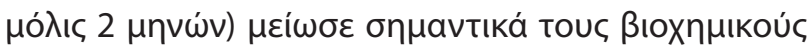

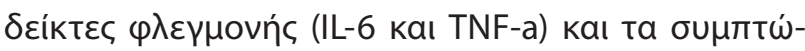

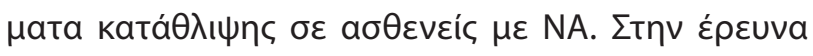

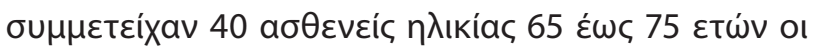

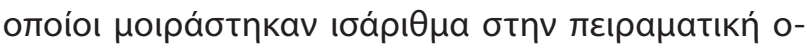

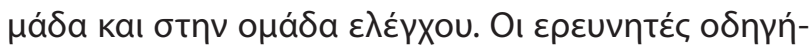

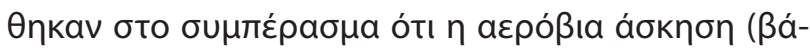

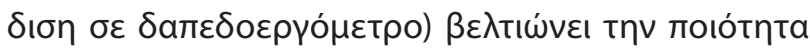

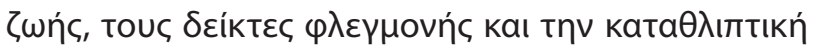

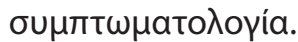

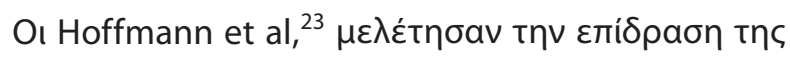

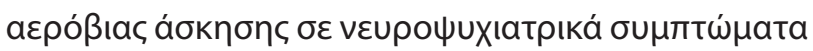

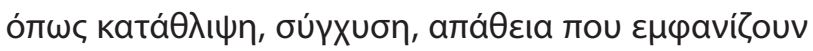

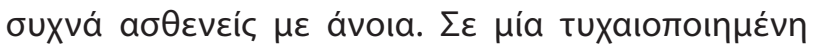

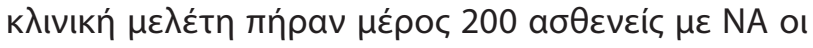

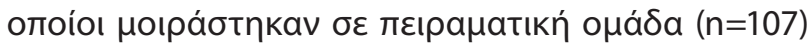

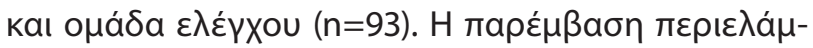

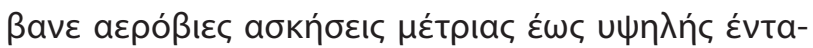

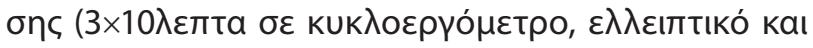

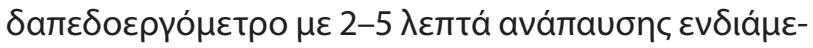

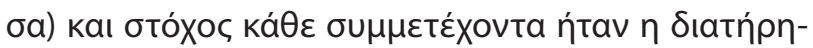

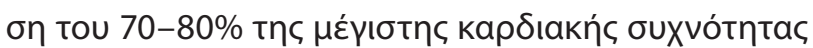

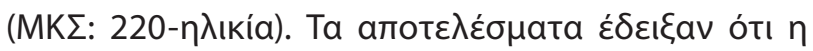

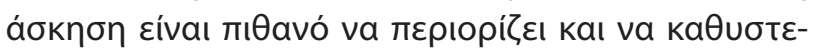

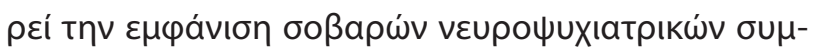

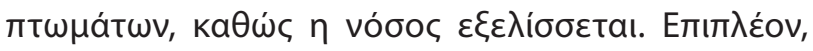

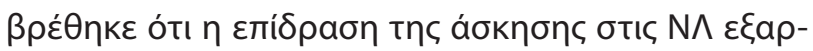

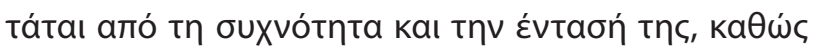

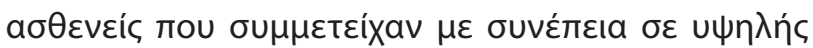

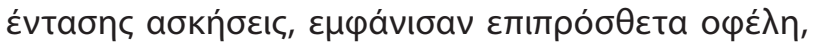

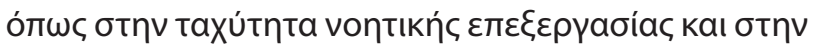
пробохи́.

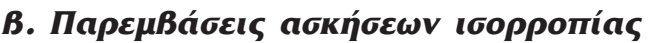

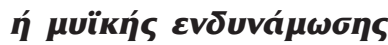

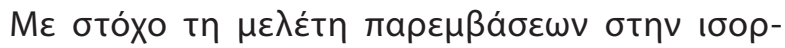

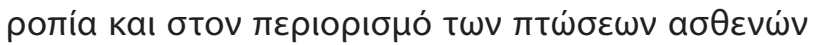

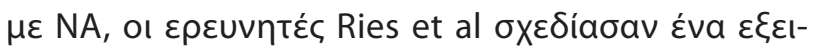

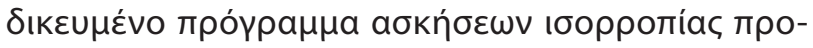

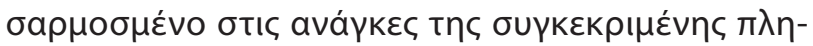

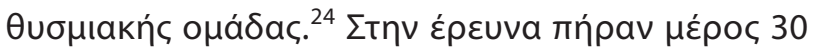

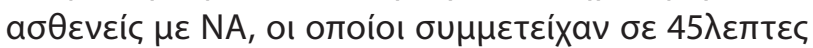

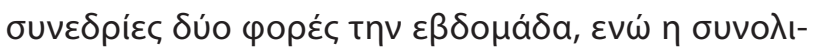

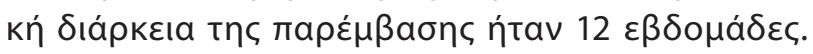

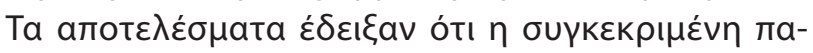

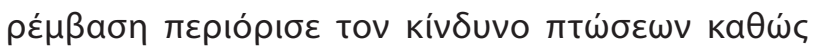

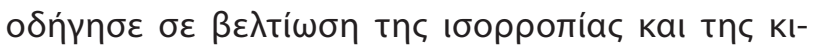

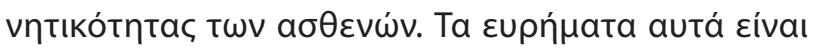

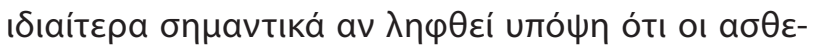

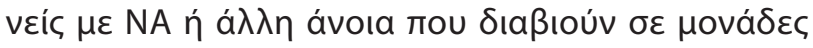




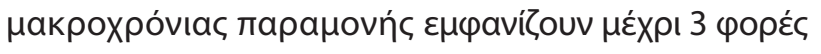

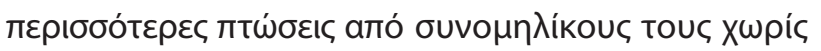

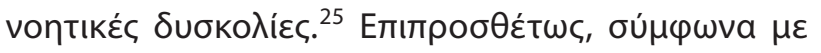

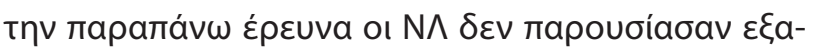

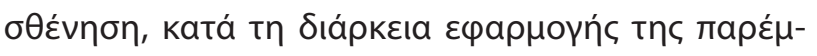

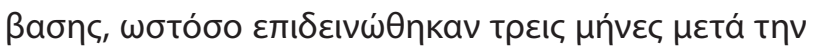

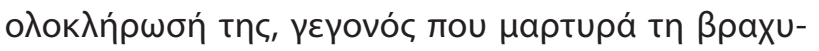

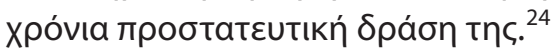

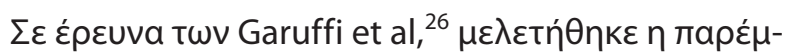

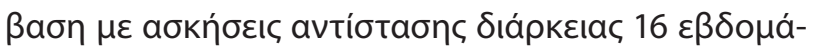

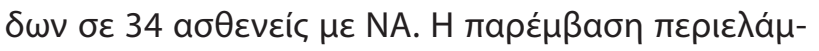

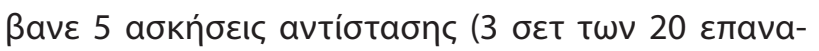

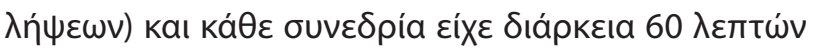

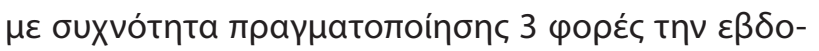

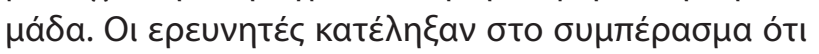

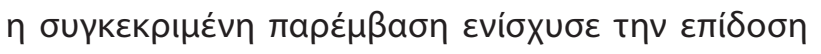

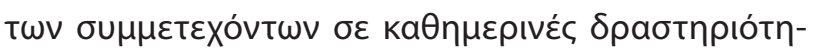

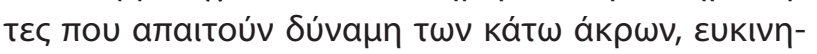

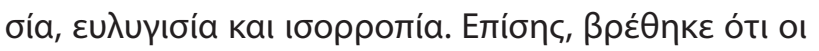

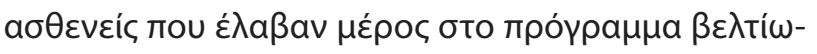

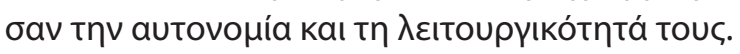

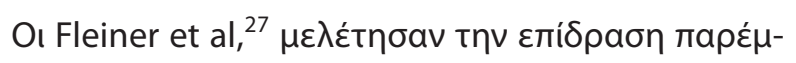

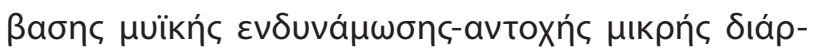

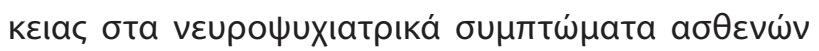

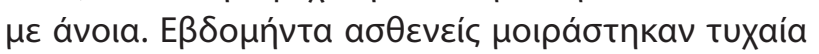

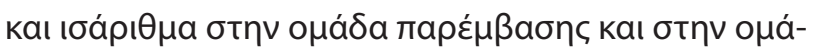

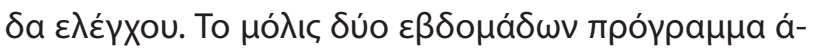

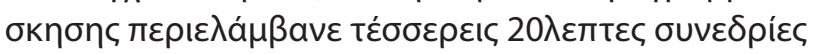

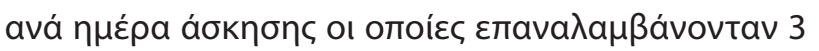

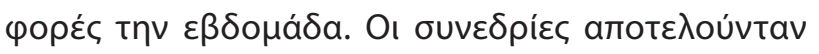

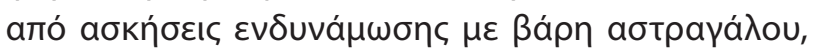

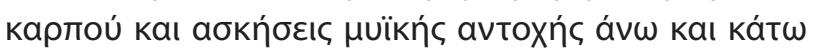

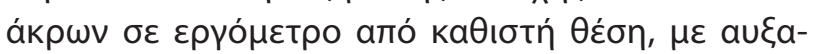

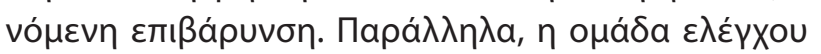

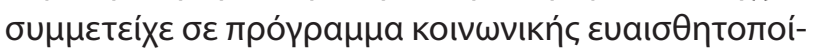

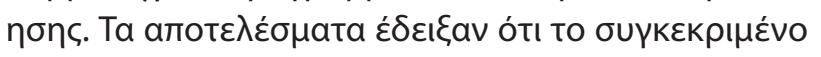

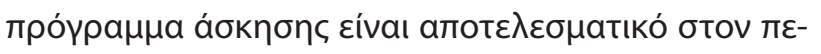

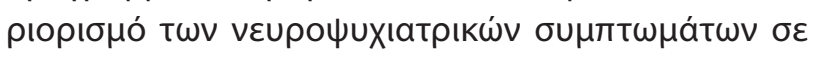

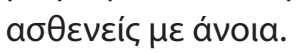

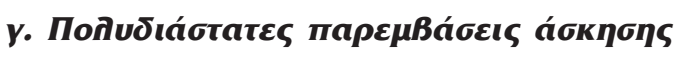

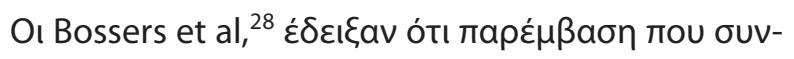

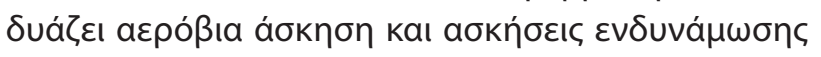

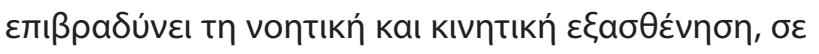

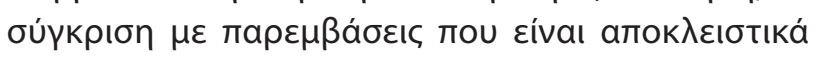

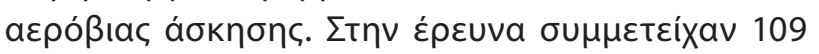

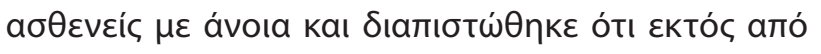

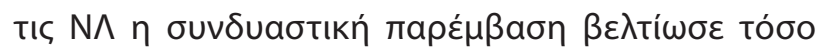

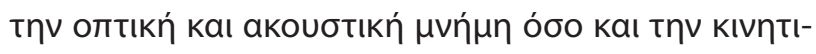

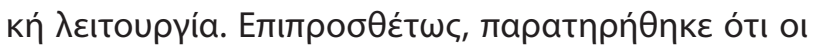

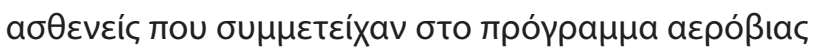

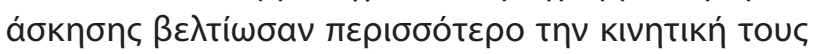

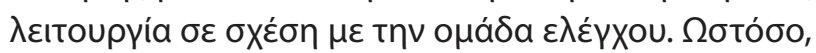

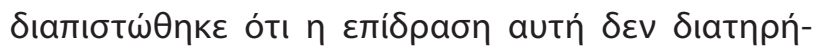

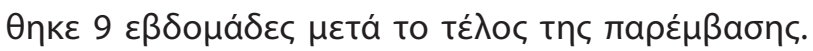

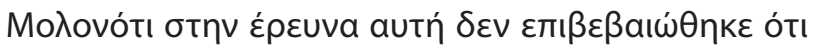

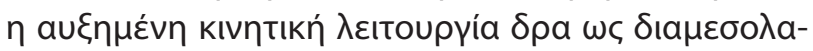

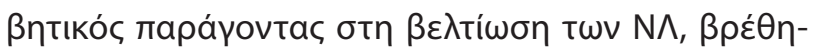

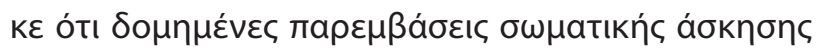

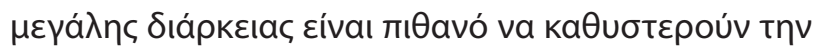

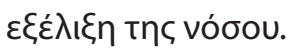

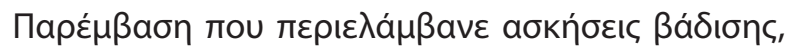

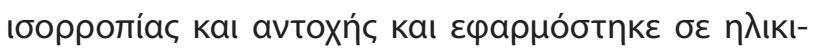

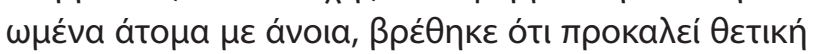

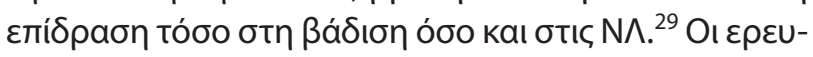

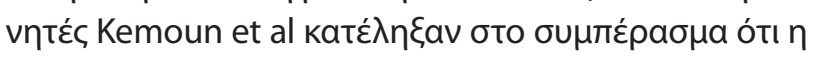

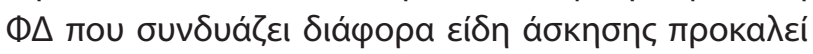

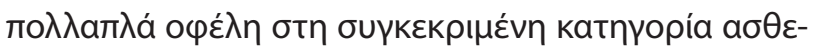

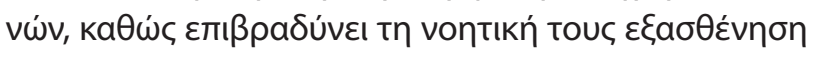

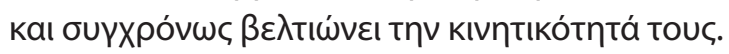

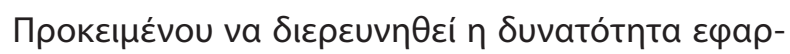

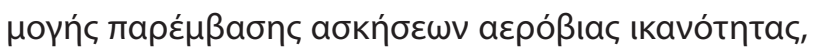

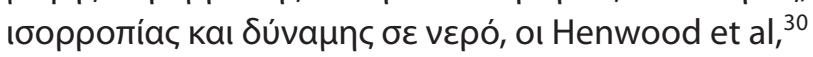

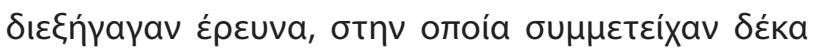

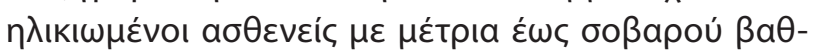

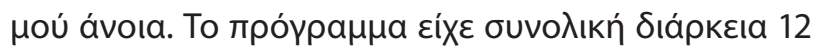

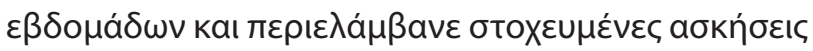

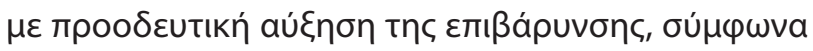

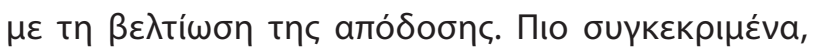

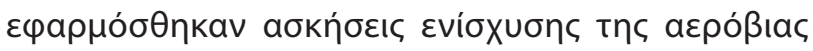

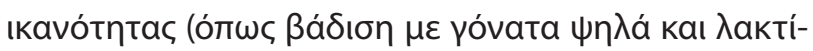

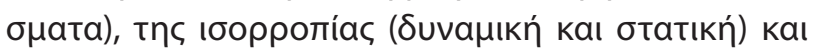

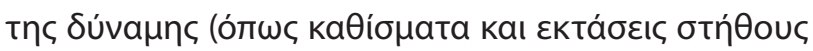

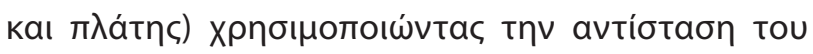

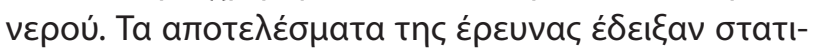

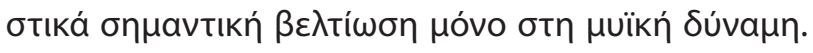

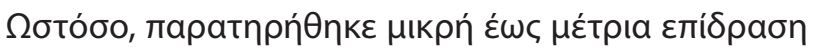

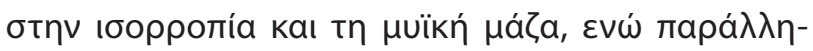

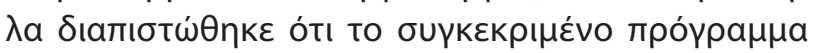

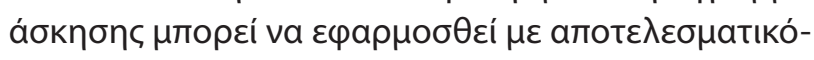

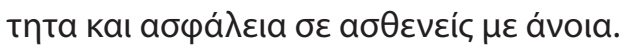

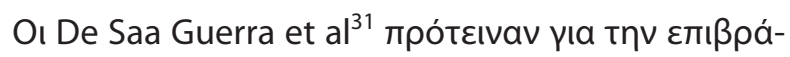

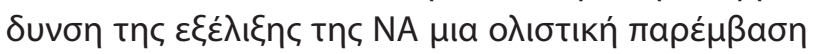

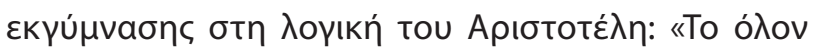




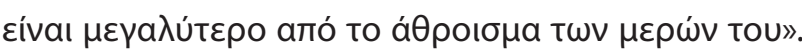

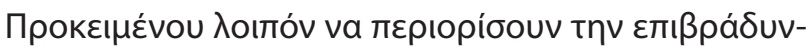

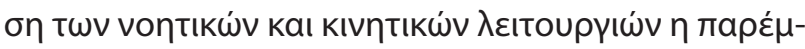

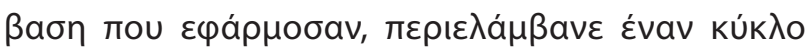

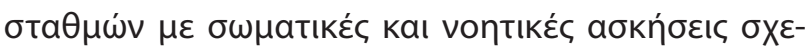

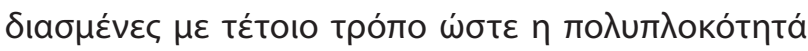

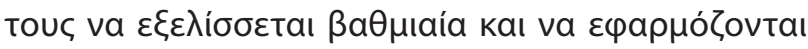

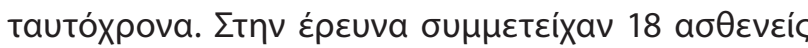

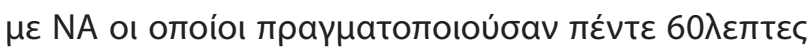

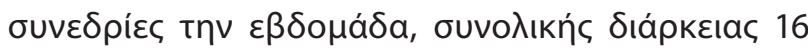

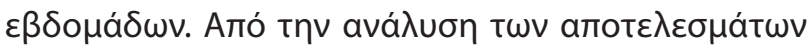

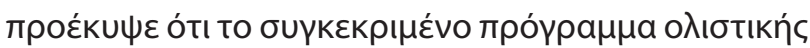

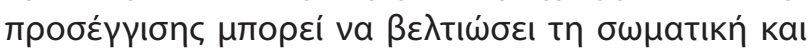

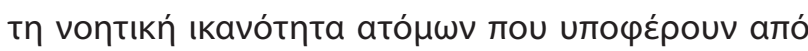

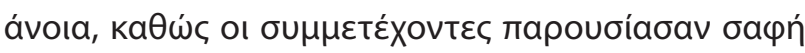

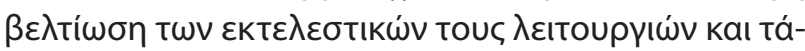

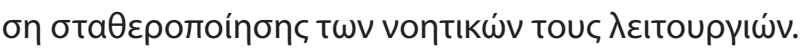

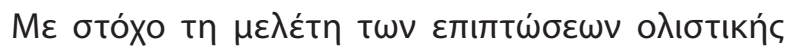

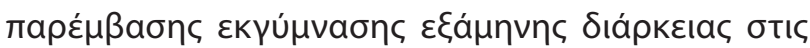

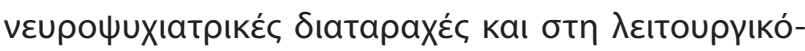

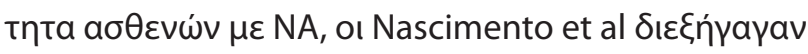

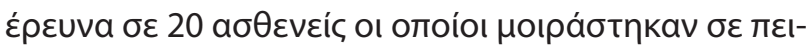

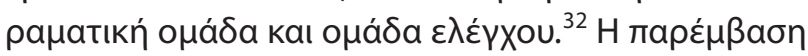

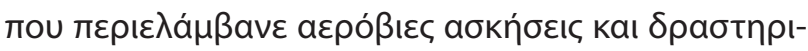

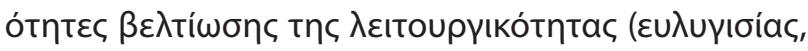

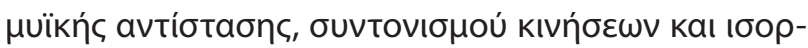

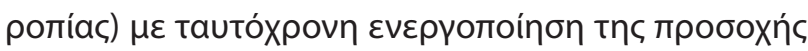

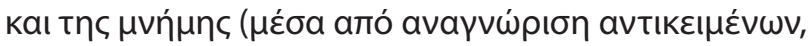

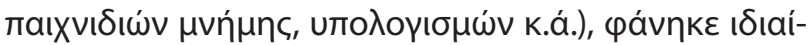

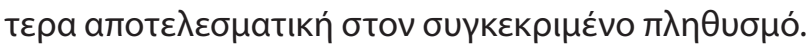

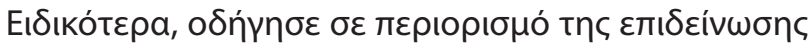

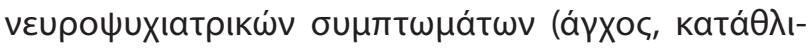

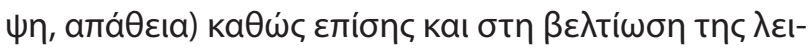

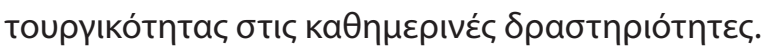

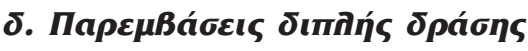

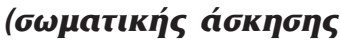

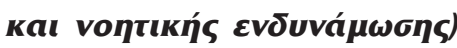

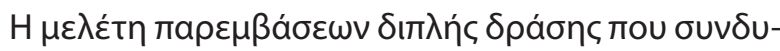

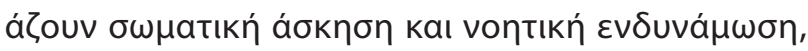

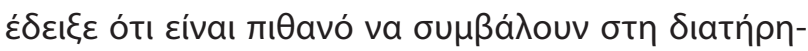

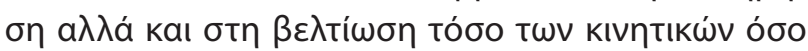

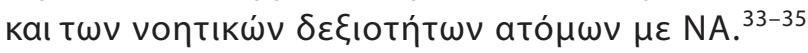

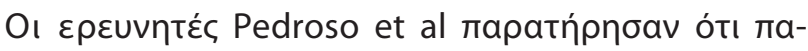

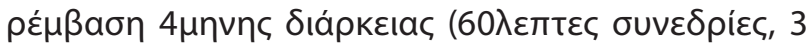

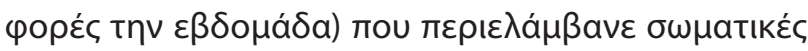

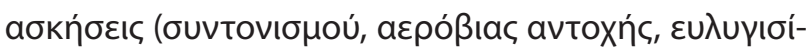

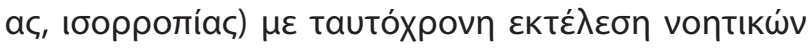

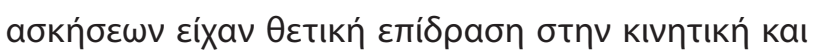

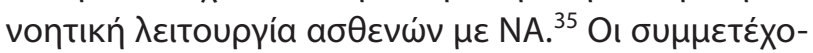

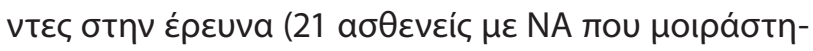

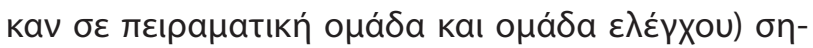

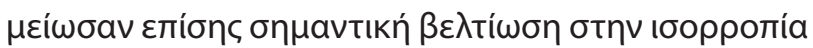

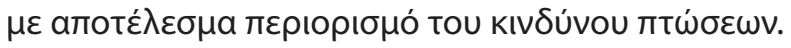

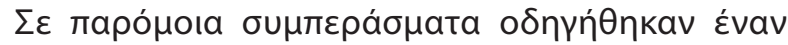

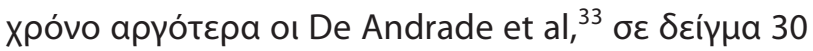

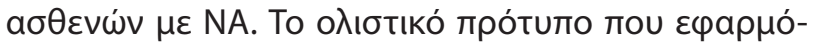

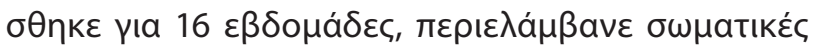

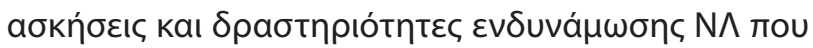

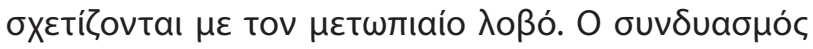

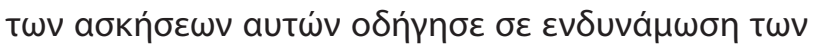

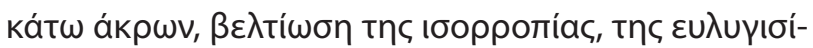

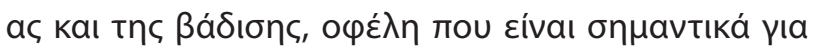

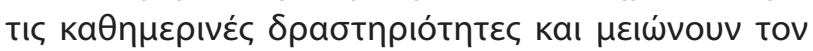

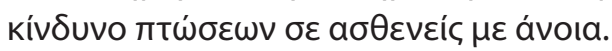

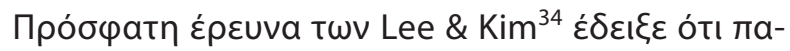

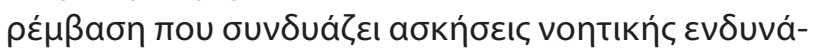

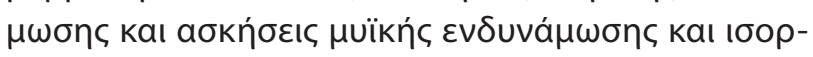

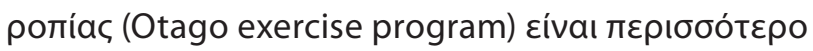

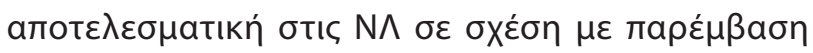

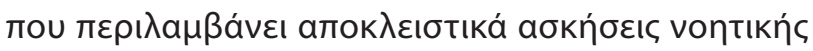

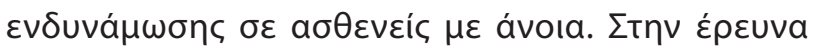

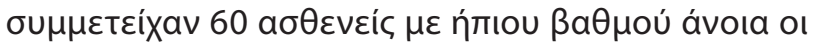

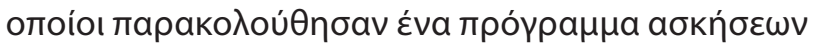

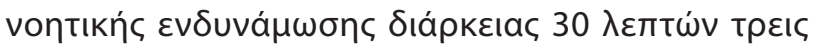

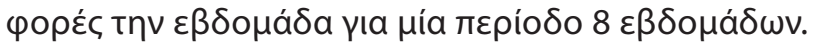

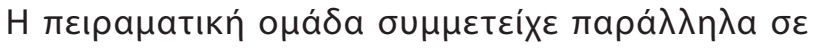

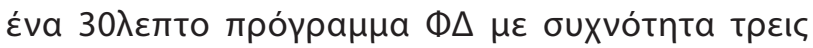

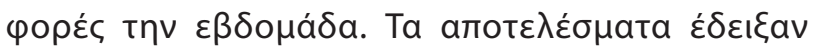

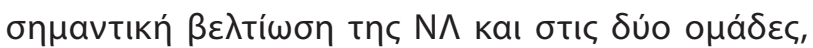

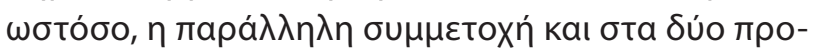

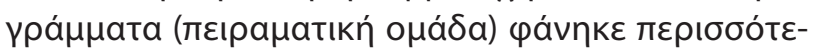

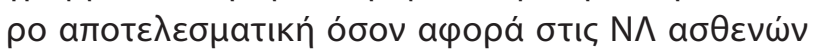

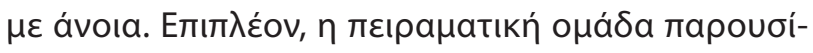

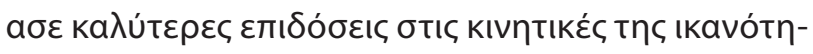

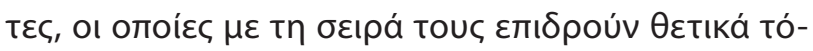

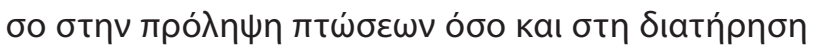

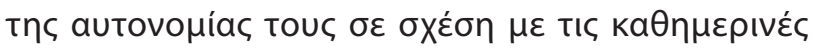

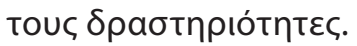

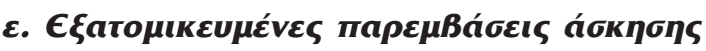 кат' оíкоv}

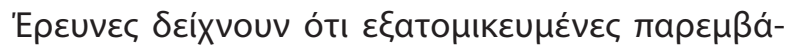

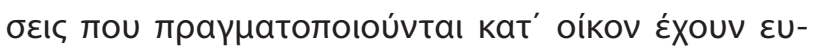

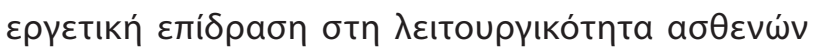




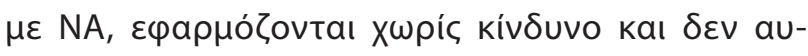

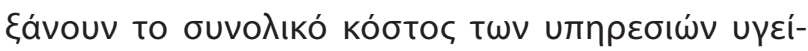

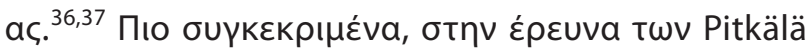

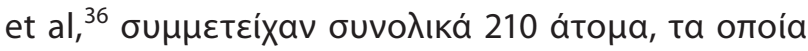

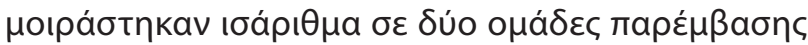

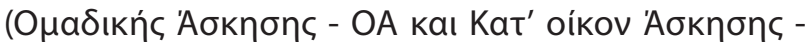

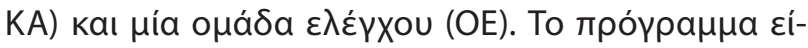

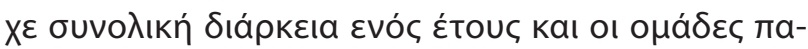

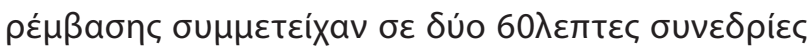

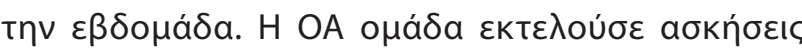

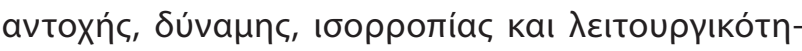

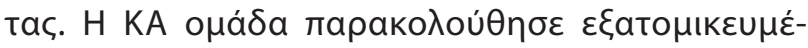

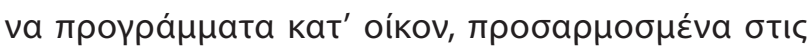

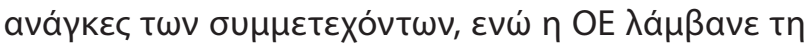

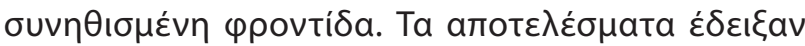

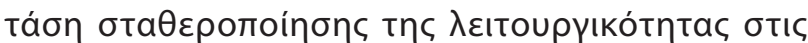
о

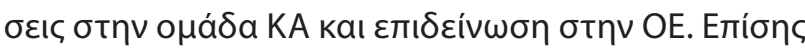

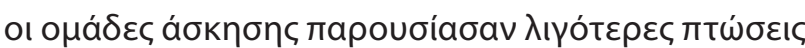

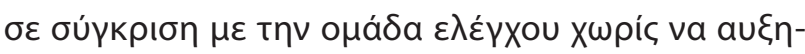

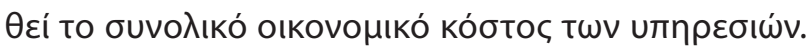

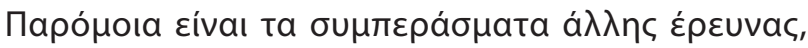

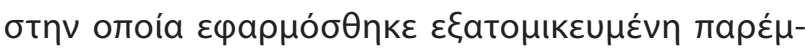

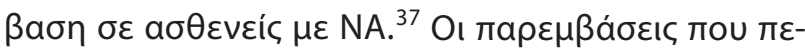

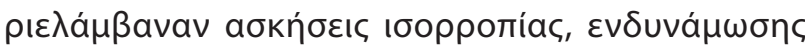

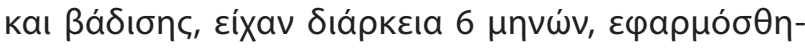

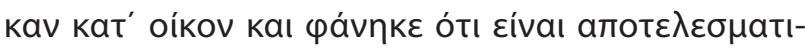

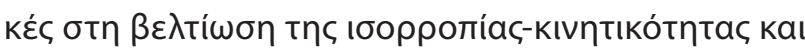

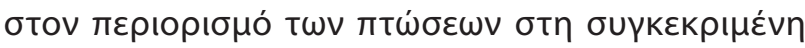

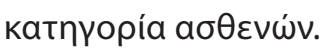

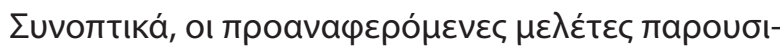

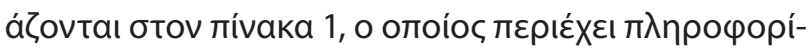

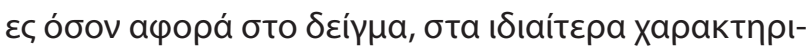

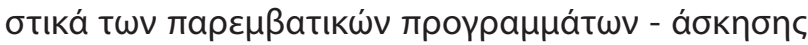

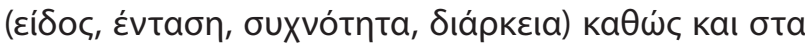

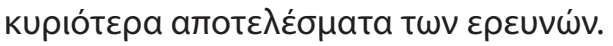

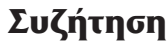

H $\pi \lambda \varepsilon ı$

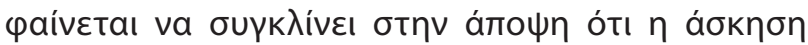

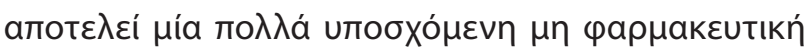

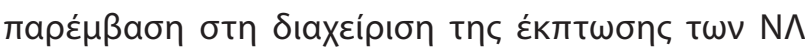

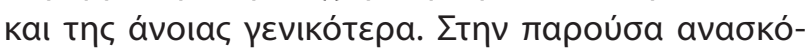

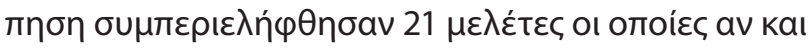

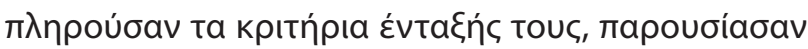

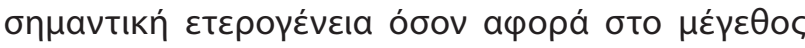

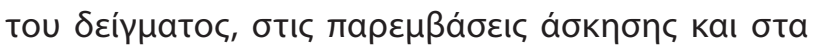

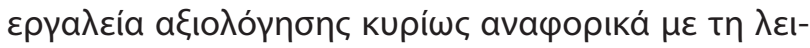

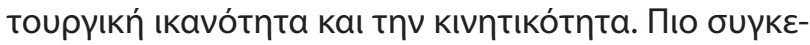

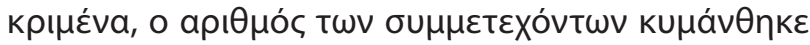

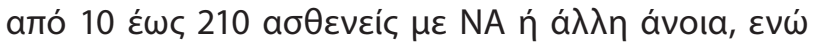

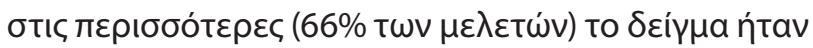

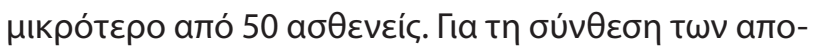

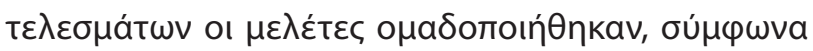

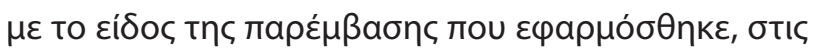

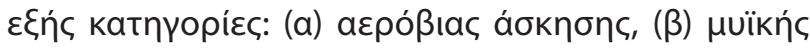

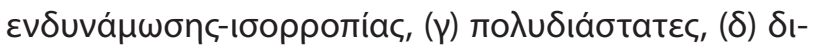

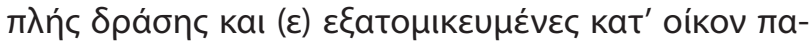

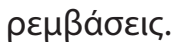

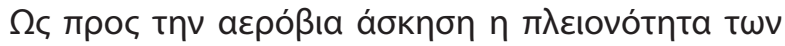

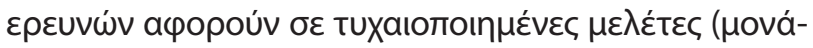

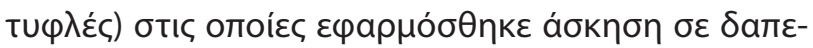

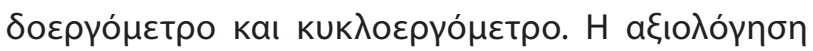

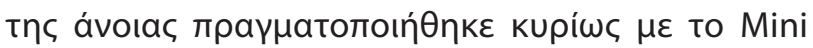
Mental State Examination (MMSE), ${ }^{16-19,21,23}$ To Clinical Demenia Rate $(C D R)^{16,20}$ Kal to NINDS-ADRDA ${ }^{16,21,23}$

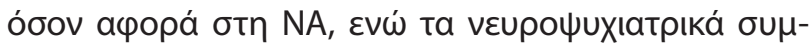

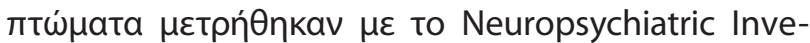

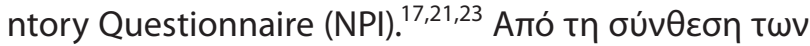

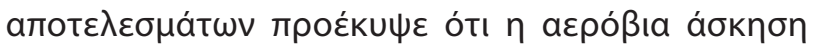

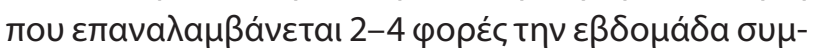

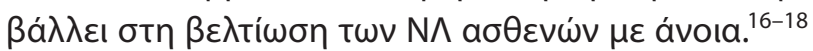

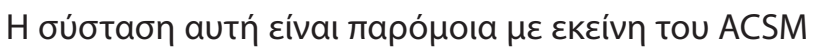
(American College of Sports Medicine) бú $\mu \varphi \omega v a \mu \varepsilon$

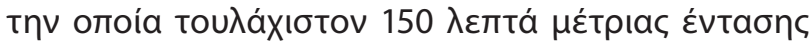

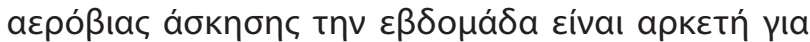

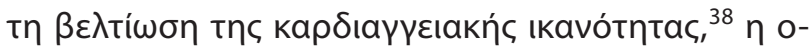

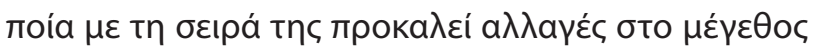

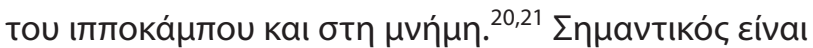

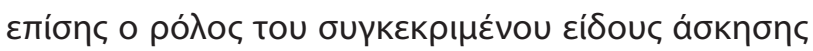

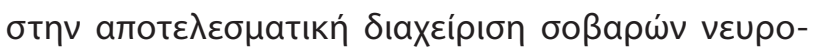

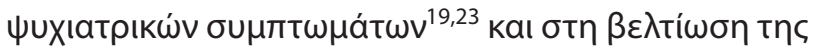

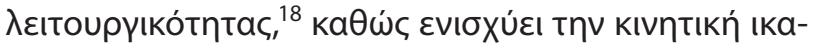
vótnta. ${ }^{16,20,21}$

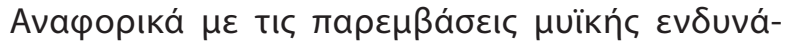

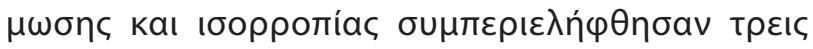

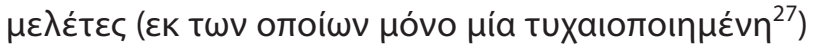

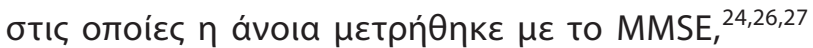
To Functional Assessment Stage Test (FAST) ${ }^{24}$ kal

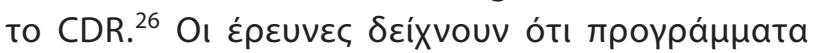

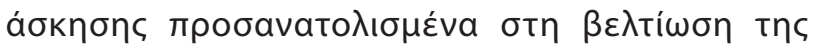

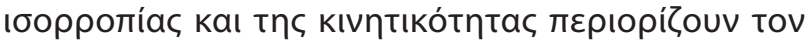




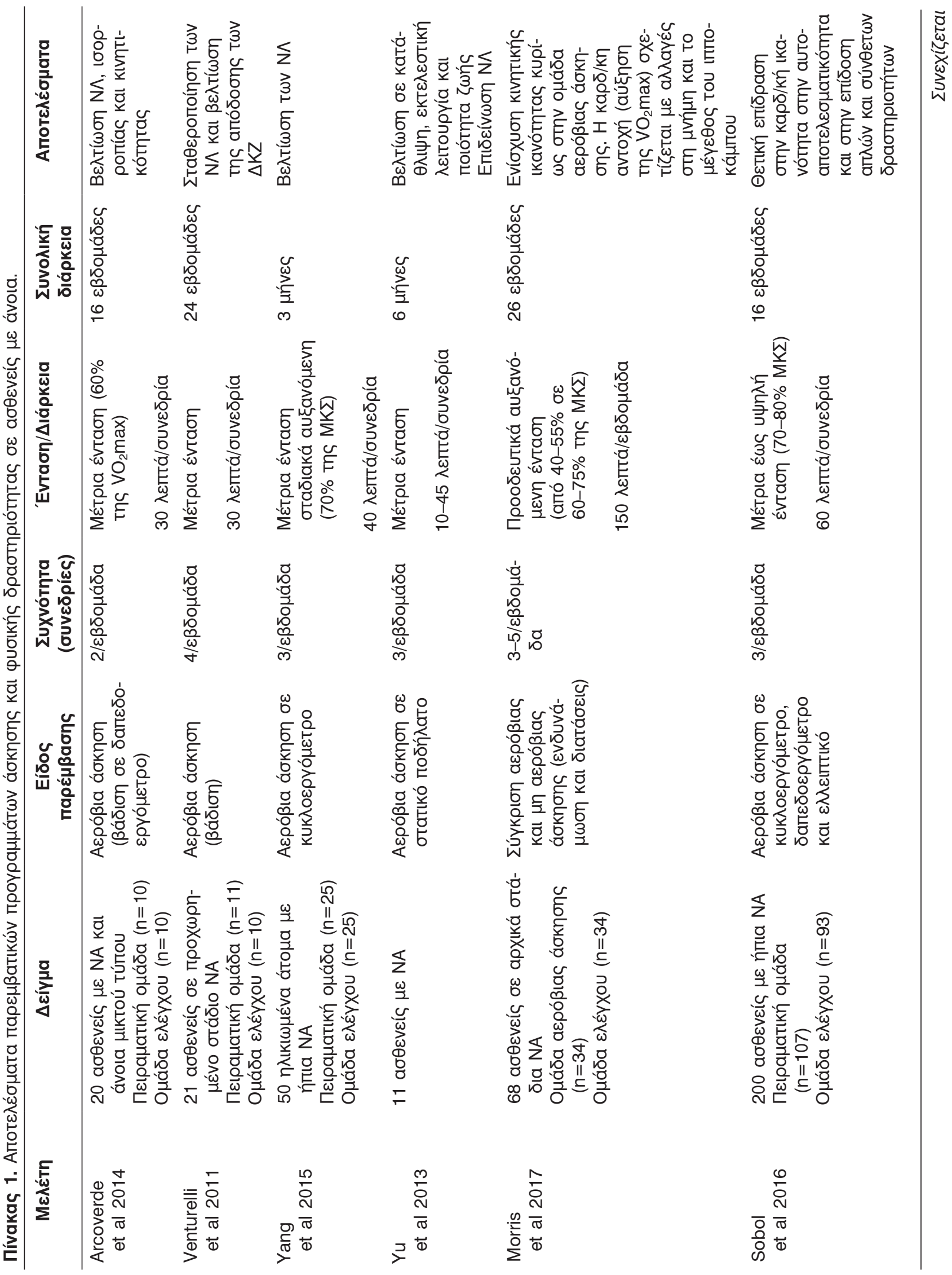




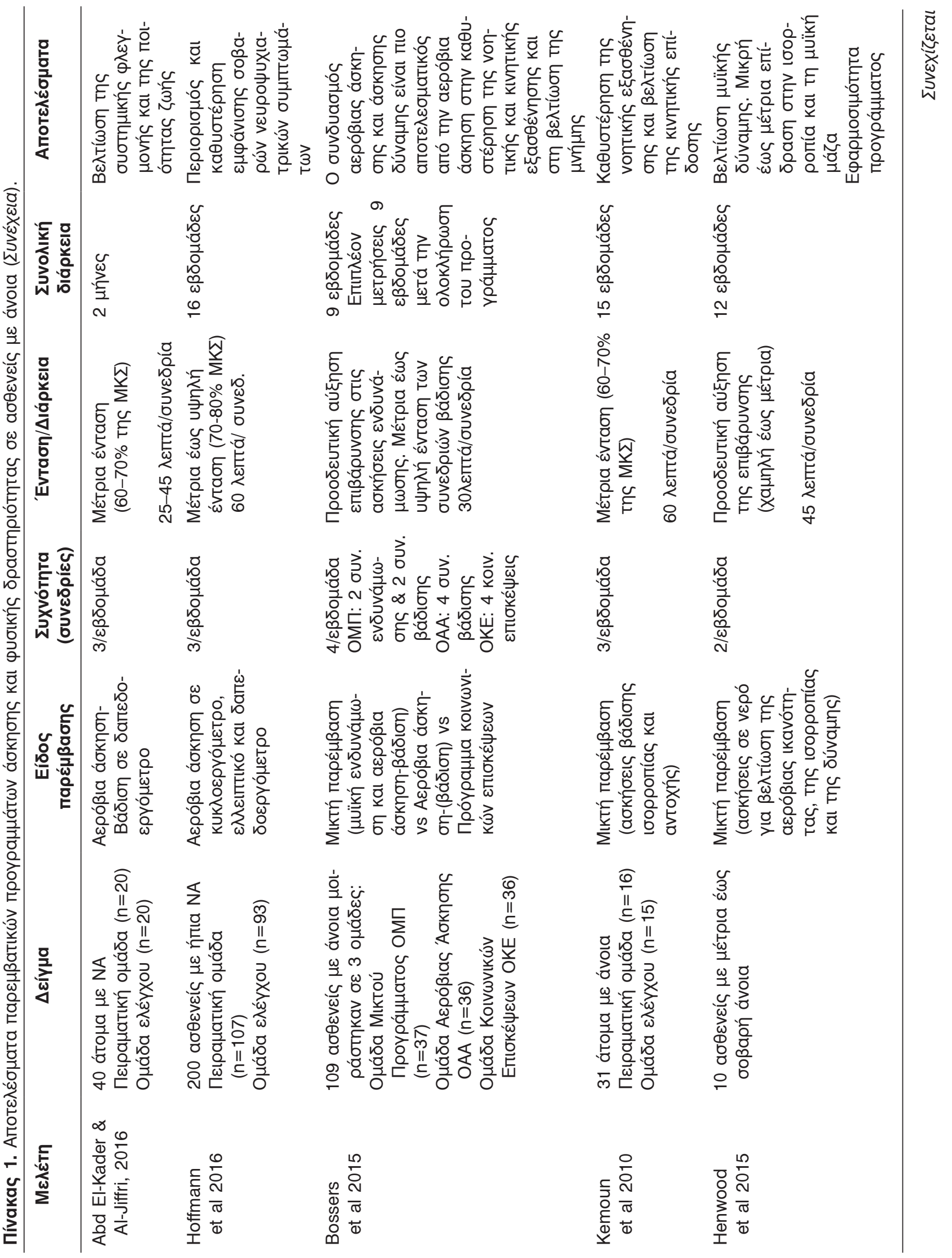




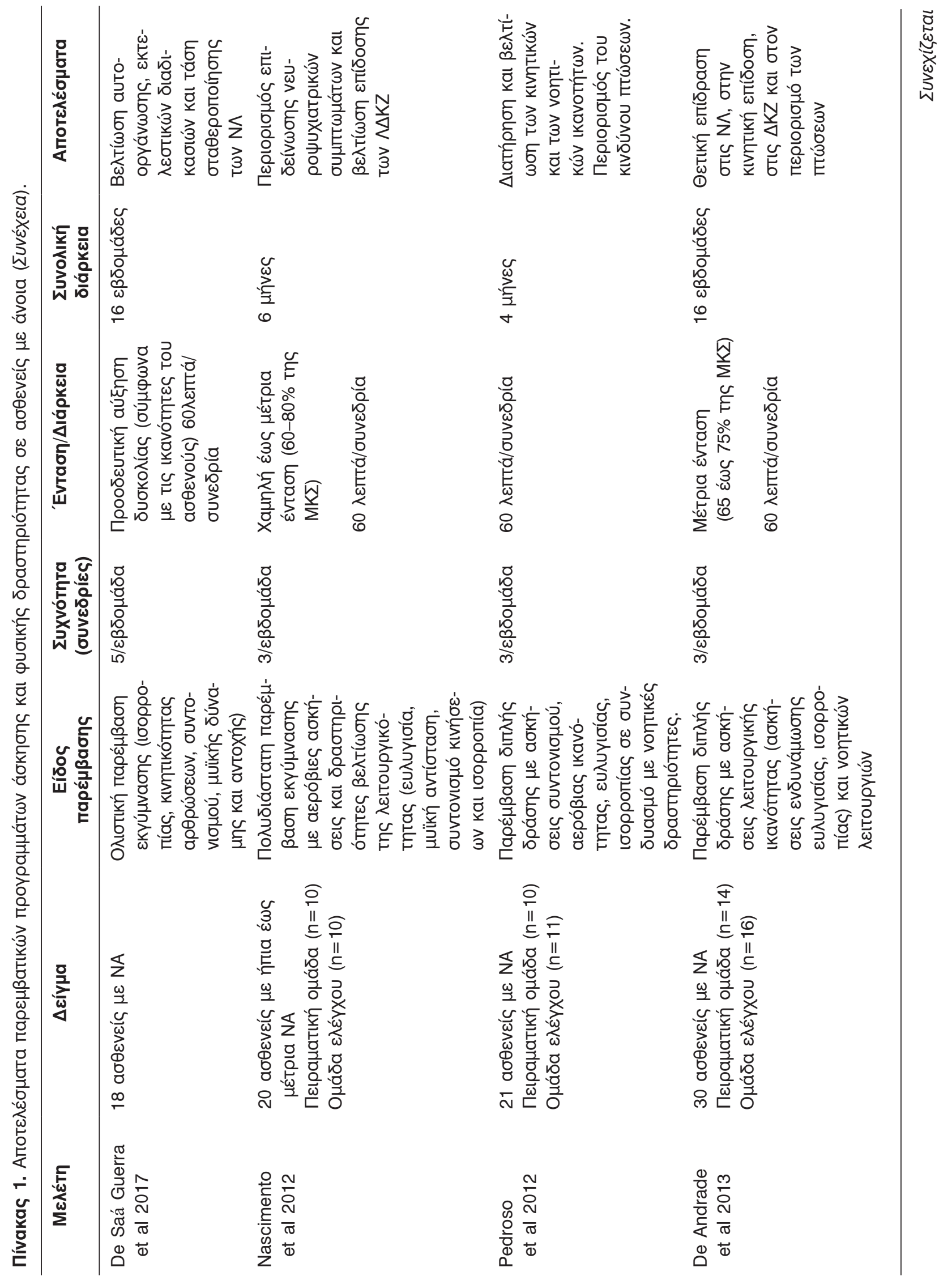




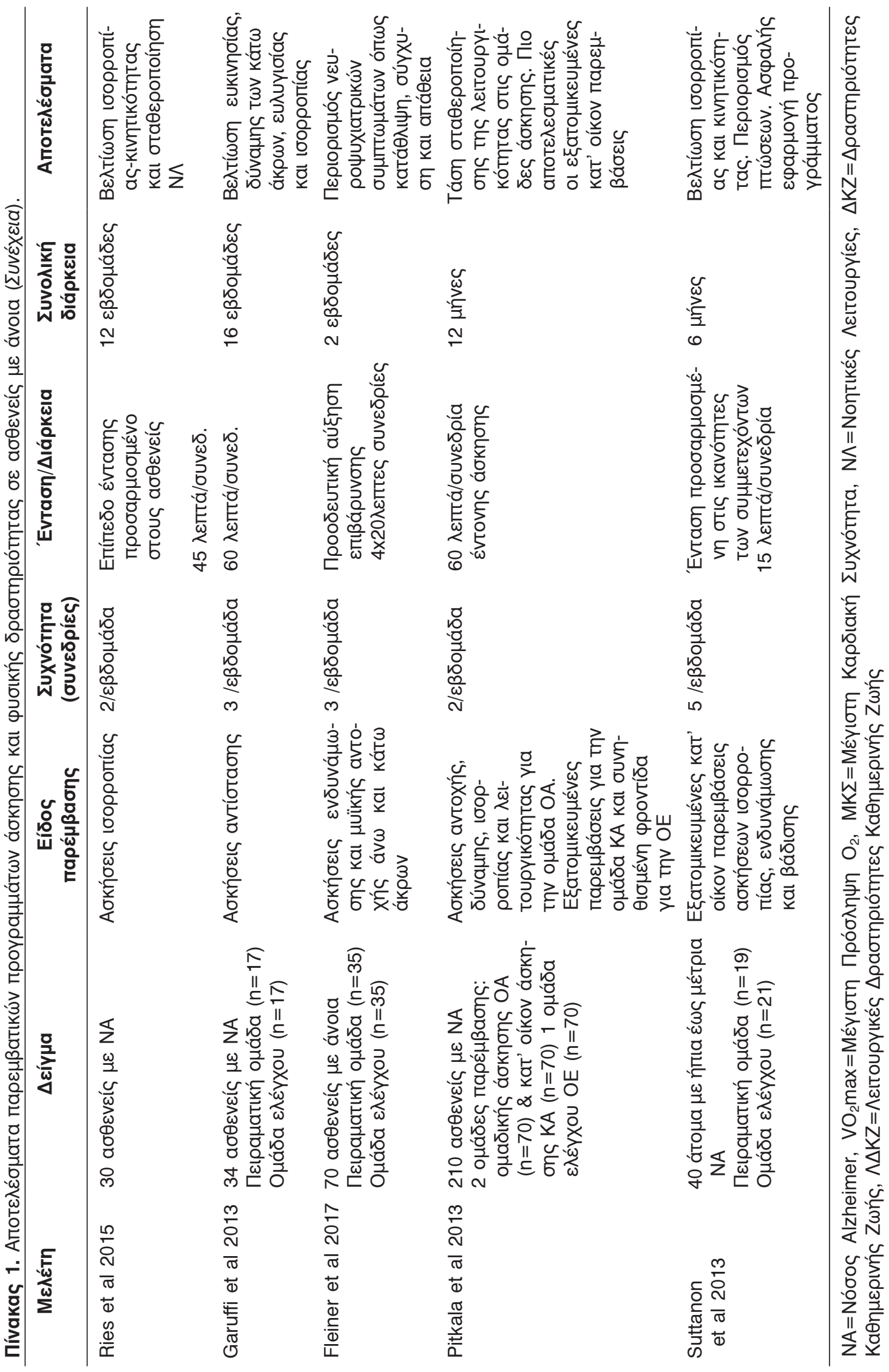




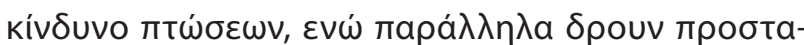

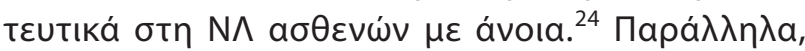

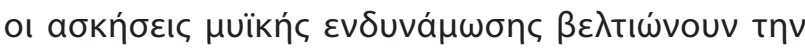

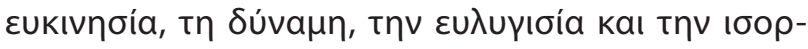

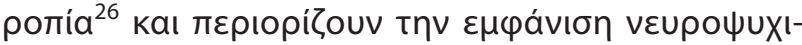

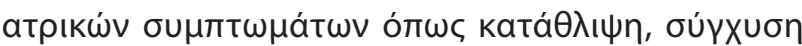

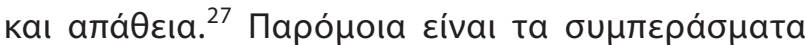

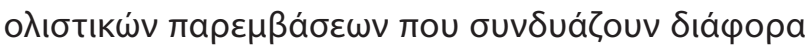

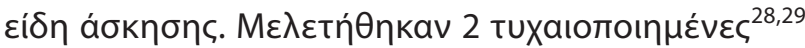

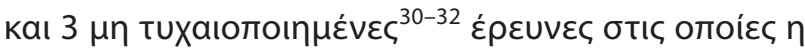

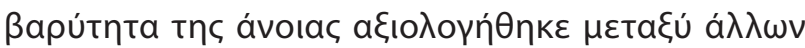
$\mu \varepsilon$ to $\mathrm{MMSE}^{28,29,31,32}$ кal to $\mathrm{FAST}^{30} \varepsilon v \omega \dot{~ t a ~ v \varepsilon u p o \psi u-~}$

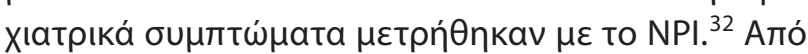

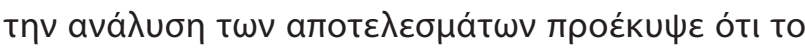

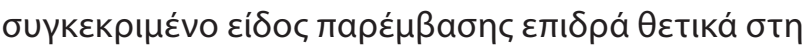

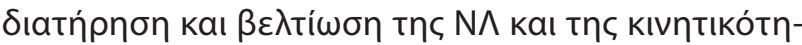

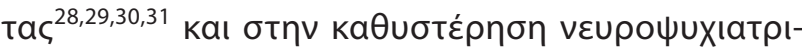

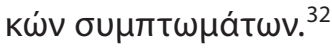

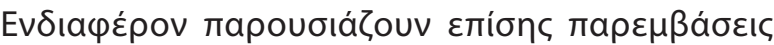

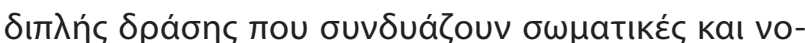

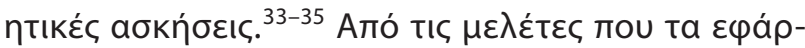

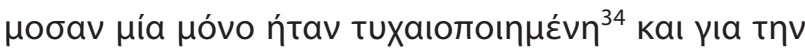

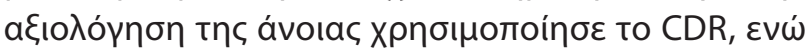

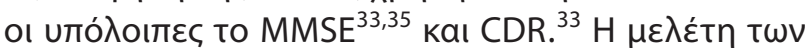

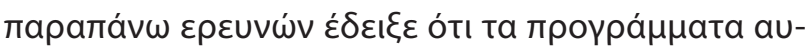

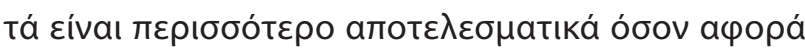

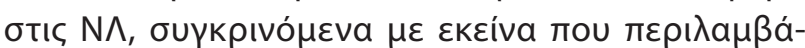

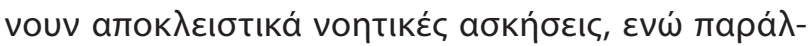

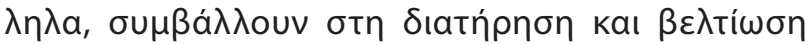

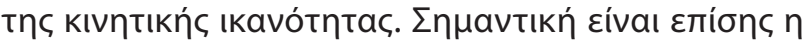

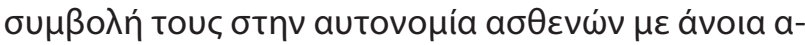

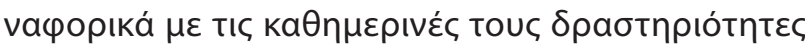

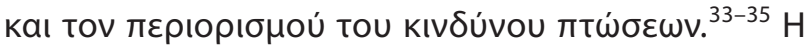

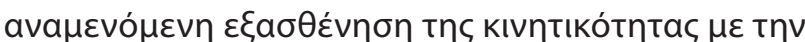

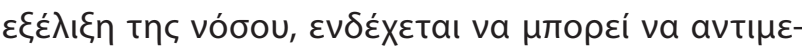

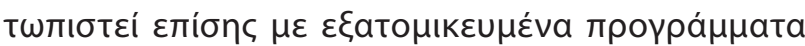

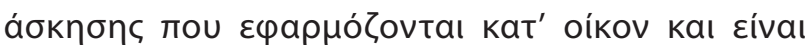

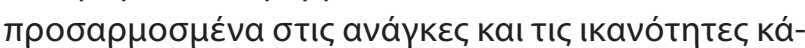

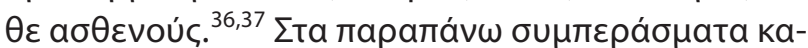

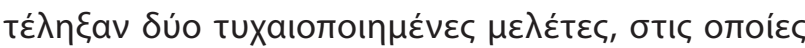

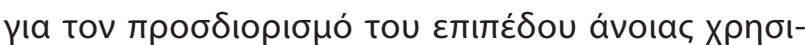

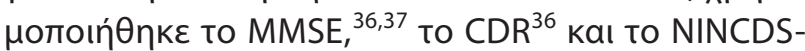
ADRDA $^{36}$ yia tn NA.

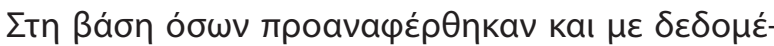

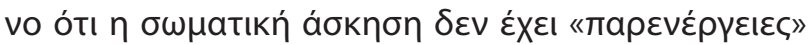

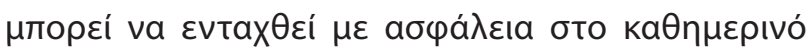

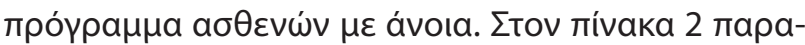

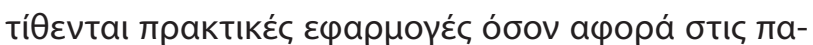

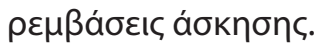

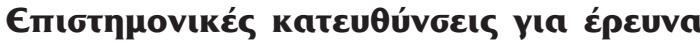

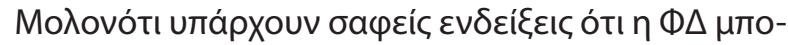

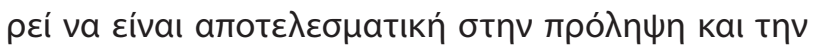

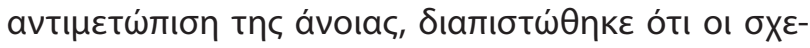

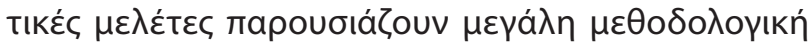

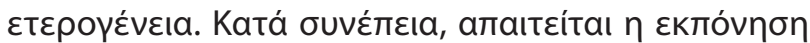

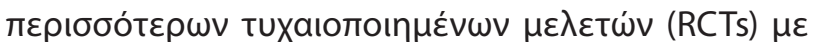

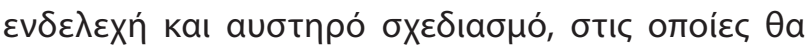

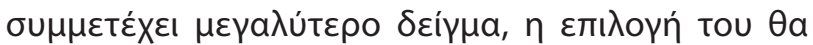

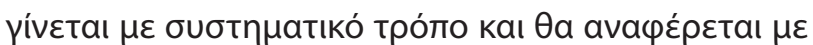

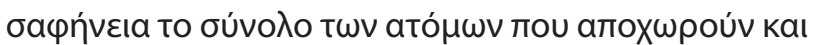

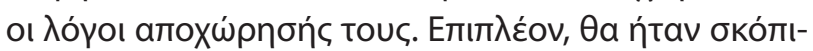

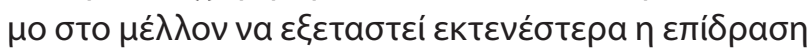

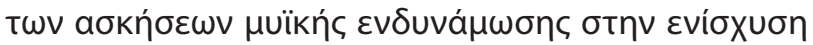

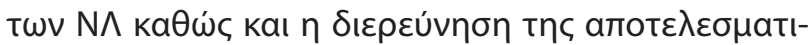

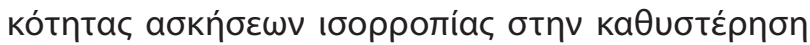

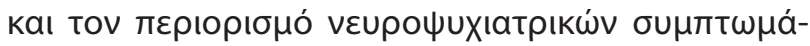

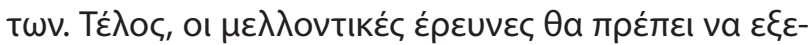

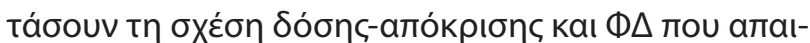

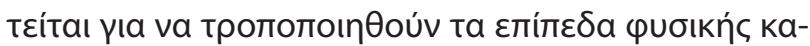

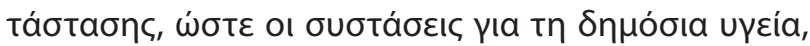

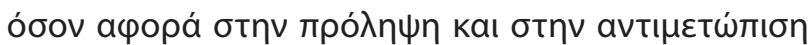

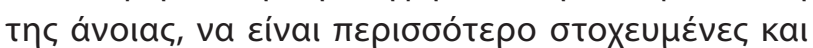

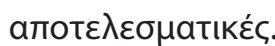

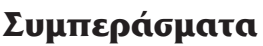

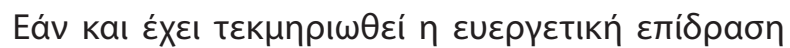

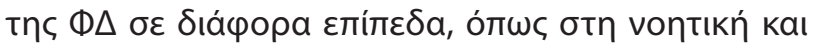

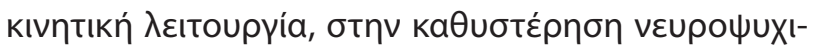

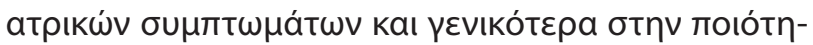

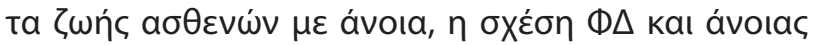

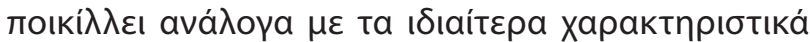

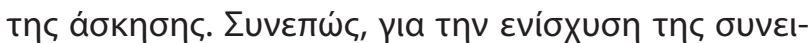

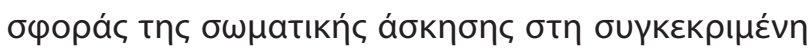

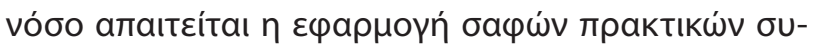

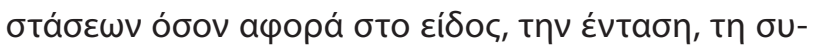

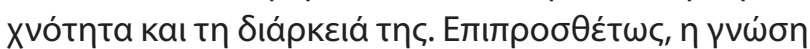

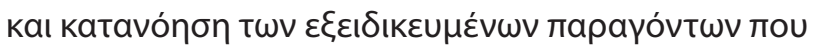
$\varepsilon \pi ı$

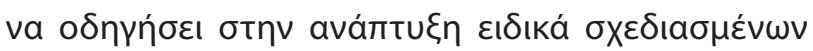

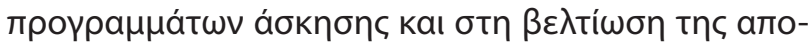

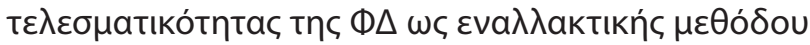

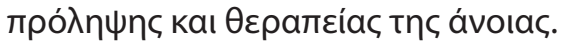




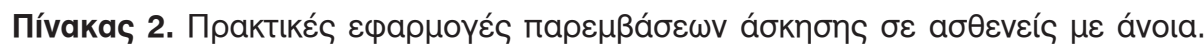

\begin{tabular}{|c|c|}
\hline 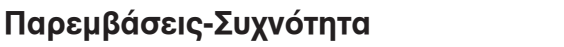 & 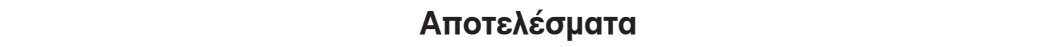 \\
\hline 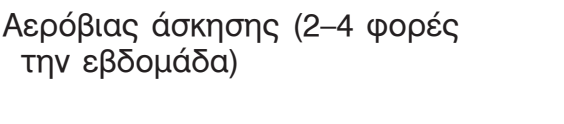 & 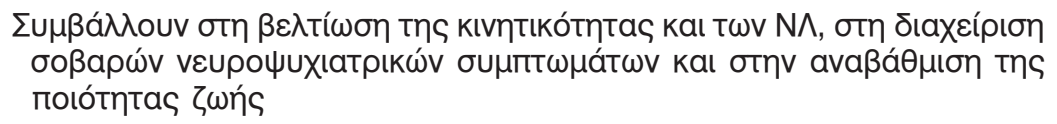 \\
\hline 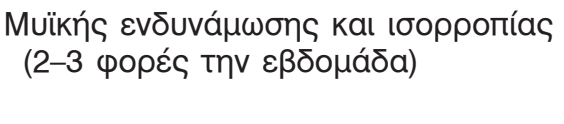 & 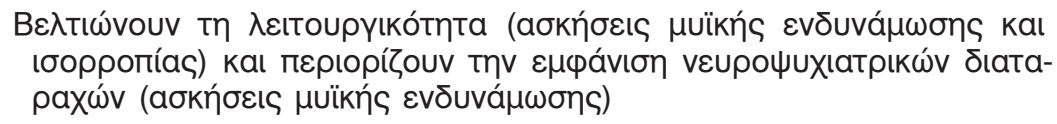 \\
\hline 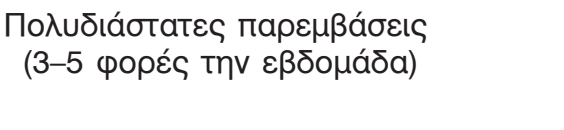 & 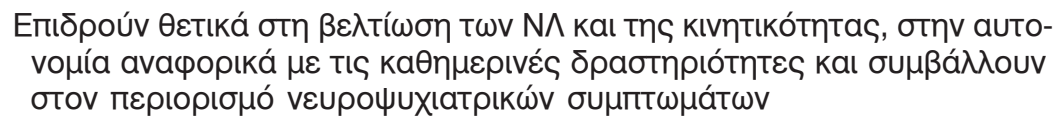 \\
\hline 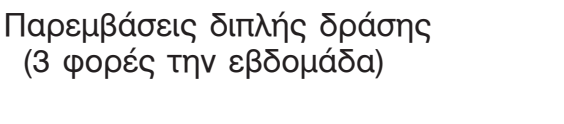 & 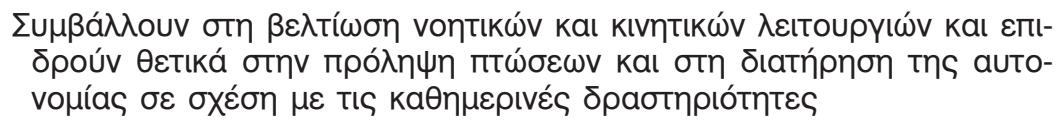 \\
\hline 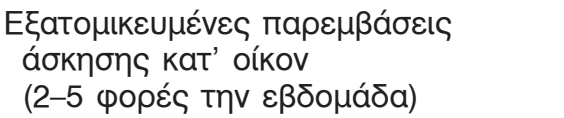 & 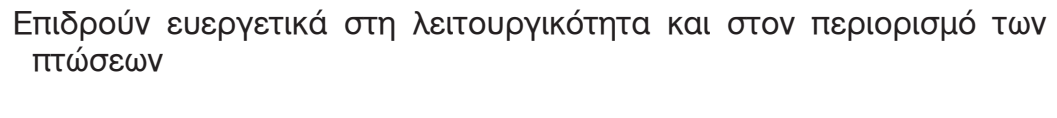 \\
\hline
\end{tabular}

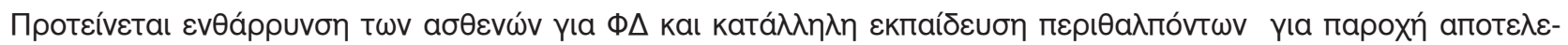

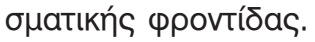

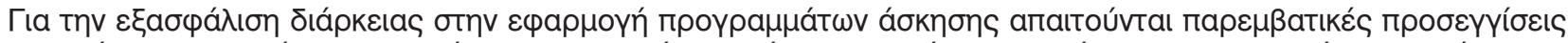

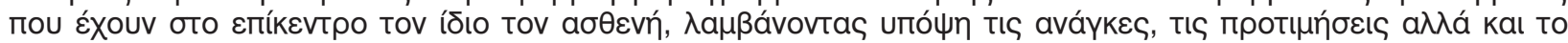

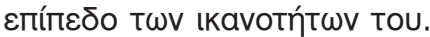

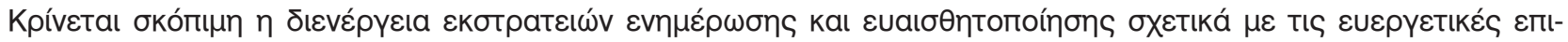

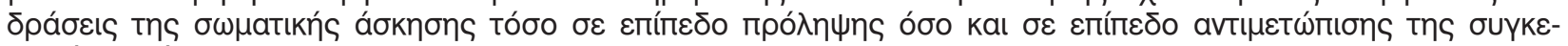

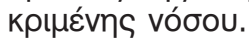

\title{
The effect of physical activity on dementia
}

\author{
K. Kouloutbani, ${ }^{1}$ K. Karteroliotis, ${ }^{1}$ A. Politis ${ }^{2}$ \\ ${ }^{1}$ School of Physical Education and Sport Science, National and Kapodistrian University of Athens, \\ ${ }^{2} 1$ st Department of Psychiatry, Eginition Hospital, National \& Kapodistrian University of Athens, Athens, Greece
}

Psychiatriki 2019, 30:142-155

The rate of patients suffering from mild cognitive impairment or any type of dementia has been constantly on the rise. Considering that no effective treatment of dementia has been discovered to date and that drug use can have numerous side effects, there is an urgent need for the application of alternative, non-pharmacological interventions. To this end, the aim of this study was to investigate the effects of physical activity on the cognitive impairment of the elderly, and its use as a form of non-pharmacological intervention for the treatment of dementia. Taking a review of the relevant literature, as its data collection method, this study examined peer-reviewed papers published between 2010 and 2018 that met the criteria for their inclusion. The articles were drawn from three electronic databases (PubMed, ScienceDirect and Web of Science), and were examined with regard to the populations under consideration, research design, type of intervention programs and assessment tools applied. The vast majority of these research papers tend to support that physical activity offers significant benefits to people suffering from Alzheimer's disease or other dementias. Specifically, it helps stabilize and improve cognitive function as well as reduce and delay the onset of 
severe neuropsychiatric symptoms such as depression, confusion, apathy, etc. In addition, physical exercise plays an important role in improving the executive functioning of patients with dementia, increasing autonomy in their everyday activities and reducing the risk of falls. In conclusion, recent research shows physical activity to be a promising intervention for the prevention and non-pharmacological treatment of dementia in that it contributes to the improvement of patients' quality of life. However, results vary according to the particularly characteristics of the exercise under review, such as type, intensity, frequency, and duration. It is therefore important to gain both awareness and understanding of the specific factors that give physical activity its therapeutic potential leading to the development of exercise programs designed specially to treat dementia.

Key words: Physical activity, physical exercise, exercise, dementia, Alzheimer's disease.

\section{Bıßntoypacpía}

1. Prince MJ, Wimo A, Guerchet MM, Ali GC, Wu Y-T, Prima M. World Alzheimer Report 2015 - The Glodal Impact of Dementia: An analysis of prevalence, incidence, cost and trends. Alzheimer's Disease International, London, 2015

2. Radak Z, Hart N, Sarga L, Koltai E, Atalay M, Ohno H et al. Exercise plays a preventive role against Alzheimer's disease. $J$ Alzheimers Dis 2010, 20:777-783, doi: 10.3233/JAD-2010091531

3. Winblad B, Amouyel P, Andrieu S, Ballard C, Brayne C, Brodaty $\mathrm{H}$ et al. Defeating Alzheimer's disease and other dementias: A priority for European science and society. Lancet Neurol 2016, 15:455-532, doi: 10.1016/S1474-4422(16)00062-4

4. World Health Organisation. Dementia. A public health priority 2012 http://apps.who.int/iris/bitstream/handle/10665/75263/ 9789241564458_eng.pdf;jsessionid=92674AA231747917DE94 E681A2244CFB?sequence $=1(31 / 07 / 2018)$

5. Kemoun G, Thibaud M, Roumagne N, Carette P, Albinet C, Toussaint $L$ et al. Effects of a physical training programme on cognitive function and walking efficiency in elderly persons with dementia. Dement Geriatr Cogn Disord 2010, 29:109-114, doi: $10.1159 / 000272435$

6. Lange-Asschenfeldt C, Kojda G. Alzheimer's disease, cerebrovascular dysfunction and the benefits of exercise: From vessels to neurons. Exp Gerontol 2008, 43:499-504, doi: 10.1016/j. exger.2008.04.002

7. Petersen RC, Lopez O, Armstrong MJ, Getchius TSD, Ganguli M, Gloss D et al. Practice guideline update summary: Mild cognitive impairment: Report of the Guideline Development, Dissemination, and Implementation Subcommittee of the American Academy of Neurology. Neurology 2018, 90:126-135, doi: 10.1212/WNL.0000000000004826

8. Steinberg M, Leoutsakos JS, Podewils LJ, Lyketsos CG. Evaluation of a home-based exercise program in the treatment of Alzheimer's disease: The Maximizing Independence in Dementia (MIND) study. Int J Geriatr Psychiatry 2009, 24:680685, doi: $10.1002 / g p s .2175$

9. Zhou Z, Fu J, Hong YA, Wang P, Fang Y. Association between exercise and the risk of dementia: results from a nationwide longitudinal study in China. BMJ Open 2017, 7:e017497, doi: 10.1136/bmjopen-2017-017497
10. Brookmeyer R, Gray S, Kawas C. Projections of Alzheimer's disease in the United States and the public health impact of delaying disease onset. Am J Public Health 1998, 88:1337-42, PMID: 9736873

11. Lenzer J. FDA warns about using antipsychotic drugs for dementia. BMJ 2005, 330:922, doi: 10.1136/bmj.330.7497.922-c

12. De Bruijn RF, Schrijvers EM, De Groot KA, Witteman JC, Hofman A, Franco $\mathrm{OH}$, et al. The association between physical activity and dementia in an elderly population: the Rotterdam Study. Eur J Epidemiol 2013, 28:277-283, doi: 10.1007/s10654-013-9773-3

13. McEwen SC, Siddarth P, Abedelsater B, Kim Y, Mui W, Wu $P$, et al. Simultaneous aerobic exercise and memory training program in older adults with subjective memory impairments. $J$ Alzheimers Dis 2018, 62:795-806, doi: 10.3233/JAD-170846

14. Plassman B, Williams JWJr, Burke JR, Holsinger T, Benjamin S. Systematic review: factors associated with risk for and possible prevention of cognitive decline in later life. Ann Intern Med 2010, 153:182-193, doi: 10.7326/0003-4819-153-3-201008030-00258

15. Williamson JD, Espeland M, Kritchevsky SB, Newman AB, King $A C$, Pahor $M$ et al. Changes in cognitive function in a randomized trial of physical activity: results of the lifestyle interventions and independence for elders pilot study. J Gerontol A Biol Sci Med Sci 2009, 64:688-694, doi: 10.1093/gerona/glp014

16. Arcoverde C, Deslandes A, Moraes H, Almeida C, de Araujo NB, Vasques PE et al. Treadmill training as an augmentation treatment for Alzheimer's disease: a pilot randomized controlled study. Arq Neuropsiquiatr 2014, 72:190-196, PMID: 24676435

17. Yang SY, Shan CL, Qing H, Wang W, Zhu Y, Yin MM et al. The effects of aerobic exercise on cognitive function of Alzheimer's disease patients. CNS Neurol Disord Drug Targets 2015, 14:1292-1297, PMID: 26556080

18. Venturelli M, Scarsini R, Schena F. Six-month walking program changes cognitive and ADL performance in patients with Alzheimer. Am J Alzheimers Dis Other Demen 2011, 26:381-388, doi: $10.1177 / 1533317511418956$

19. Yu F, Nelson NW, Savik K, Wyman JF, Dysken M, Bronas UG. Affecting cognition and quality of life via aerobic exercise in Alzheimer's disease. West J Nurs Res 2013, 35:24-38, doi: 10.1177/0193945911420174

20. Morris JK, Vidoni ED, Johnson DK, Van Sciver A, Mahnken JD, Honea RA et al. Aerobic exercise for Alzheimer's disease: A 
randomized controlled pilot trial. Plos One 2017, 12:e0170547, doi: 10.1371/journal.pone.0170547

21. Sobol NA, Hoffmann K, Frederiksen KS, Vogel A, Vestergaard $\mathrm{K}$, Brændgaard $\mathrm{H}$ et al. Effect of aerobic exercise on physical performance in patients with AD. Alzheimers Dement 2016, 12:1207-1215, doi: 10.1016/j.jalz.2016.05.004

22. Abd El-Kader SM, Al-Jiffri OH. Aerobic exercise improves quality of life, psychological well-being and systemic inflammation in subjects with Alzheimer's disease. Afr Health Sci 2016, 16:10451055, doi: 10.4314/ahs.v16i4.22

23. Hoffmann K, Sobol NA, Frederiksen KS, Beyer N, Vogel A, Vestergaard $\mathrm{K}$ et al. Moderate-to-high intensity physical exercise in patients with Alzheimer's disease: A randomized controlled trial. J Alzheimers Dis 2016, 50:443-453, doi: 10.3233/ JAD-150817

24. Ries JD, Hutson J, Maralit LA, Brown MB. Group balance training specifically designed for individuals with Alzheimer disease: Impact on Berg Balance Scale, Timed Up and Go, Gait Speed, and Mini-Mental Status Examination. J Geriatr Phys Ther 2015, 38:183-193, doi: 10.1519/JPT.00000000000000030

25. Shaw FE. Falls in cognitive impairment and dementia. Clin Geriatr Med 2002, 18:159-173, PMID: 12180241

26. Garuffi M, Costa JLR, Hernández SSS, Vital TM, Stein AM, dos Santos JG, Stella F. Effects of resistance training on the performance of activities of daily living in patients with Alzheimer's disease. Geriatr Gerontol Int 2013, 13:322-328, doi: 10.1111/j.1447-0594.2012.00899.x

27. Fleiner T, Dauth H, Gersie M, Zijlstra W, Haussermann P. Structured physical exercise improves neuropsychiatric symptoms in acute dementia care: A hospital-based RCT. Alzheimers Res Ther 2017, 9:68, doi: 10.1186/s13195-017-0289-z

28. Bossers WJ, van der Woude LH, Boersma F, Hortobagyi T, Scherder EJ, van Heuvelen MJ. A nine-week aerobic and strength training program improves cognitive and motor function in patients with dementia: a randomized, controlled trial. Am J Geriatr Psychiatry 2015, 23:1106-1116, doi: 10.1016/j. jagp.2014.12.191

29. Kemoun G, Thibaud M, Roumagne N, Carette P, Albinet C, Toussaint $L$ et al. Effects of a physical training programme on cognitive function and walking efficiency in elderly persons with dementia. Dement Geriatr Cogn Disord 2010, 29:109-114, doi: $10.1159 / 000272435$

30. Henwood T, Neville C, Baguley C, Clifton K, Beattie E. Physical and functional implications of aquatic exercise for nursing home residents with dementia. Geriatr Nurs 2015, 36:35-39, doi: 10.1016/j.gerinurse.2014.10.009

31. De Saa Guerra Y, Montesdeoca SS, Manso JMG, González JMM, Valdivielso MN, Ruiz DR et al. Exercise and Alzheimer's:
The body as a whole. Rev Andal Med Deporte 2017, 10:120-124, doi: org/10.1016/j.ramd.2015.11.002

32. Nascimento CMC, Teixeira CVL, Gobbi LTB, Gobbi S, Stella F. A controlled clinical trial on the effects of exercise on neuropsychiatric disorders and instrumental activities in women with Alzheimer's disease. Rev Bras Fisioter 2012, 16:197-204, doi: org/10.1590/S1413-35552012005000017

33. De Andrade LP, Gobbi LT, Coelho FG, Christofoletti G, Costa JL, Stella F. Benefits of multimodal exercise intervention for postural control and frontal cognitive functions in individuals with Alzheimer's disease: a controlled trial. J Am Geriatr Soc 2013, 61:1919-1926, doi: 10.1111/jgs.12531

34. Lee HJ, Kim KD. Effect of physical activity on cognition and daily living activities of the elderly with mild dementia. J Phys Ther Sci 2018, 30:428-433, doi: 10.1589/jpts.30.428

35. Pedroso RV, Coelho FG, Santos-Galduroz RF, Costa, JLR, Gobbi S, Stella F. Balance, executive functions and falls in elderly with Alzheimer's disease (AD): A longitudinal study. Arch Gerontol Geriatr 2012, 54:348-351, doi: 10.1016/j.archger.2011.05.029

36. Pitkälä KH, Pöysti MM, Laakkonen ML, Tilvis RS, Savikko N, Kautiainen $\mathrm{H}$ et al. Effects of the Finnish Alzheimer disease exercise trial (FINALEX): a randomized controlled trial. JAMA Intern Med 2013, 173:894-901, doi: 10.1001/jamainternmed.2013.359

37. Suttanon P, Hill KD, Said CM, Williams SB, Byrne KN, LoGiudice $D$ et al. Feasibility, safety and preliminary evidence of the effectiveness of a home-based exercise programme for older people with Alzheimer's disease: a pilot randomized controlled trial. Clin Rehabil 2013, 27:427-438, doi: 10.1177/0269215512460877

38. Nelson ME, Rejeski WJ, Blair SN, Duncan PW, Judge JO, King $A C$ et al. Physical activity and public health in older adults: recommendation from the American College of Sports Medicine and the American Heart association. Med Sci Sports Exerc 2007, 39:1435-1445, doi: 10.1249/mss.0b013e3180616aa2

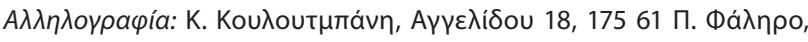

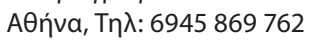

e-mail:komanthi@gmail.com 


\title{
Eıठıкó á $\theta$ $\theta$ o Special article
}

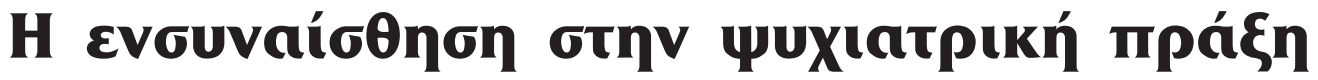

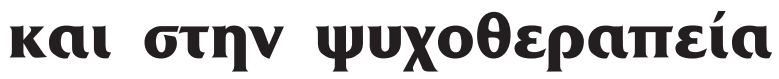

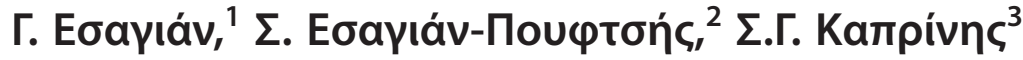

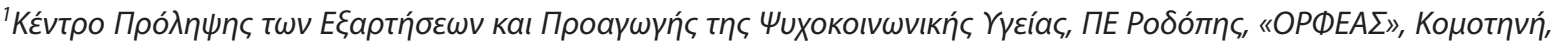

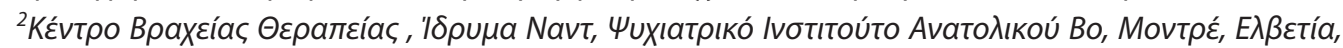

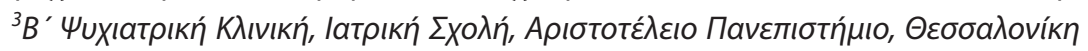 \\ Uuxıатрıкń 2019, 30:156-164
}

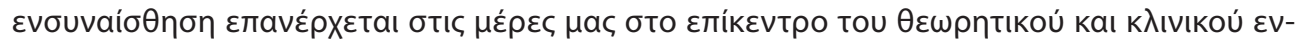

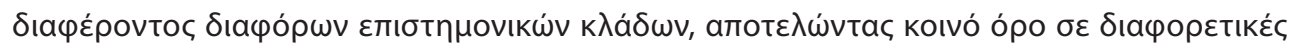

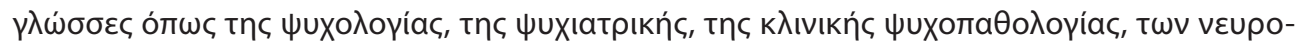

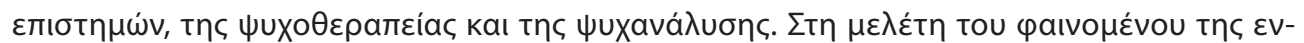

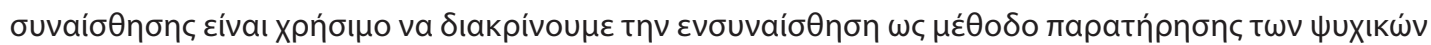

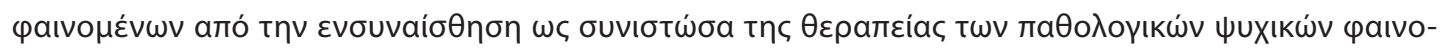

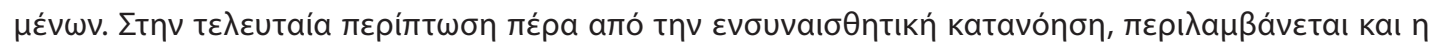

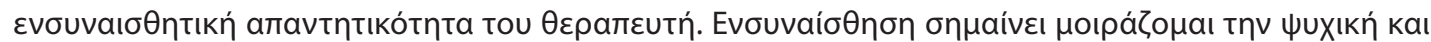

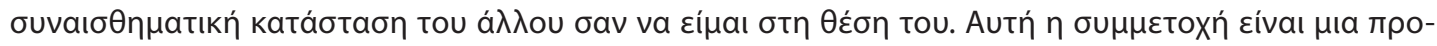

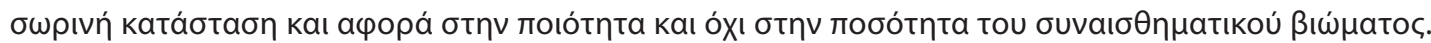

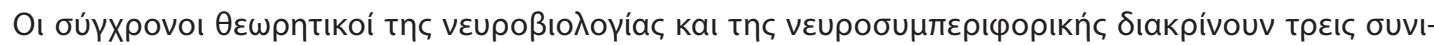

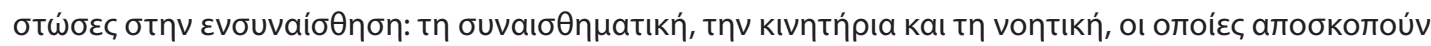

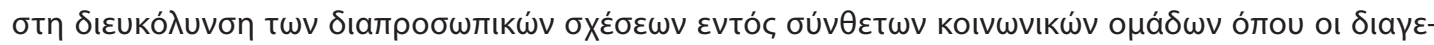

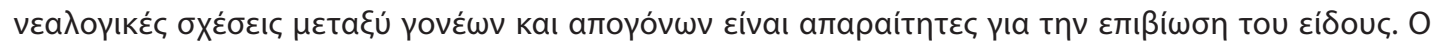

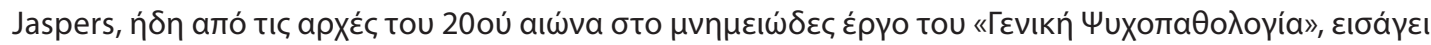

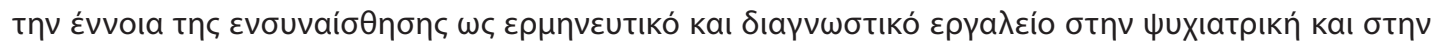

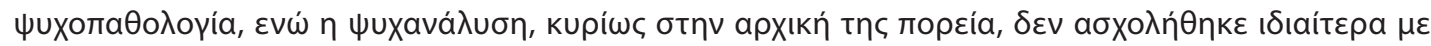

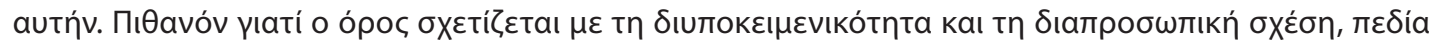

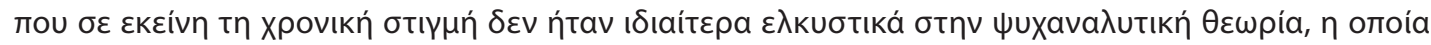

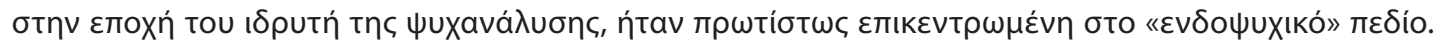

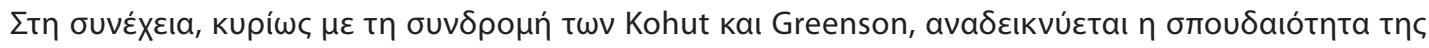

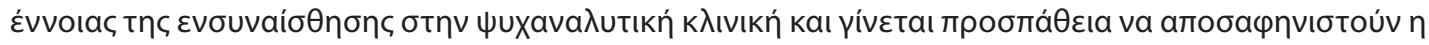




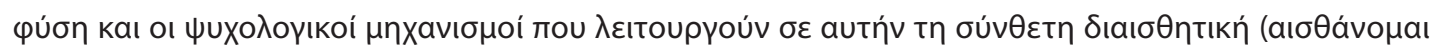

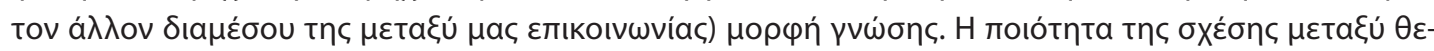

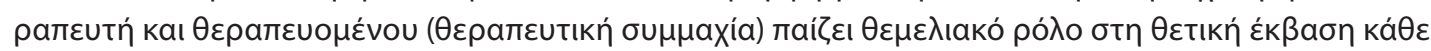

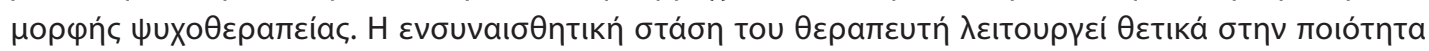

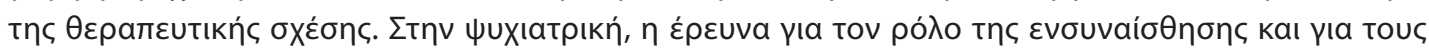

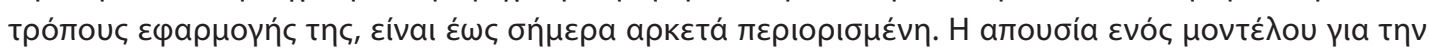

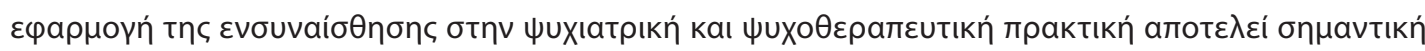

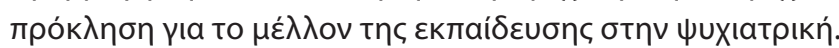

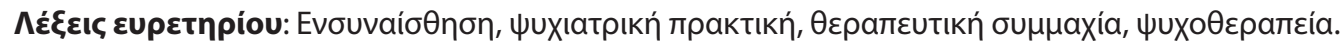

\section{Eıaaywyń}

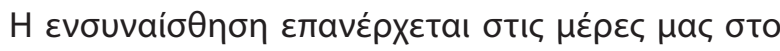

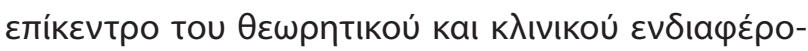

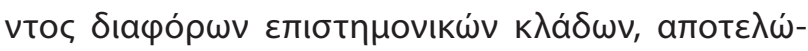

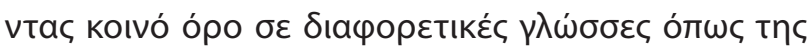

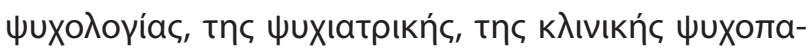

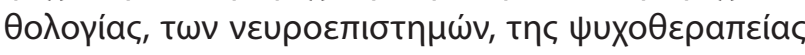

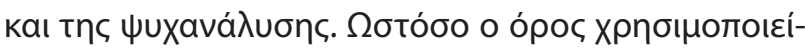

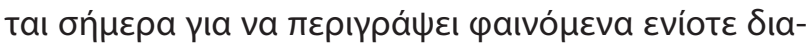

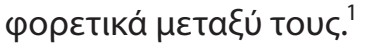

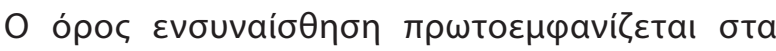

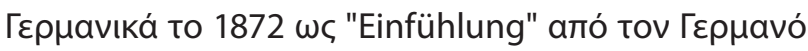

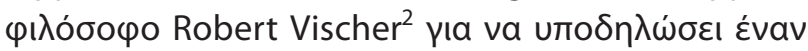

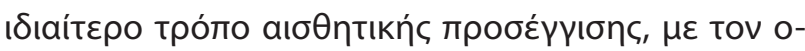

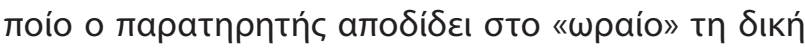

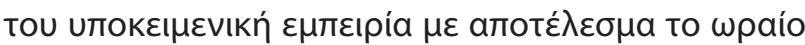

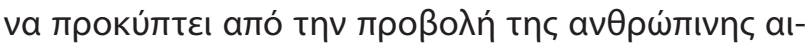

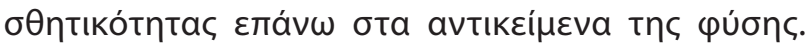

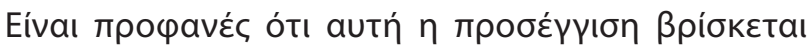

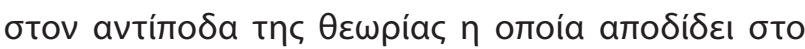

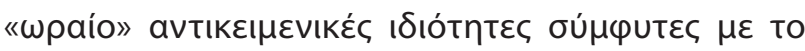

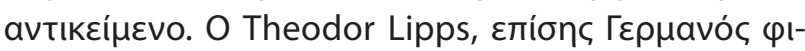

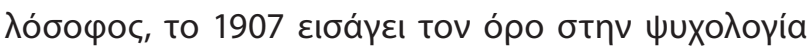

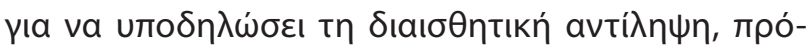

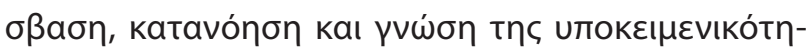

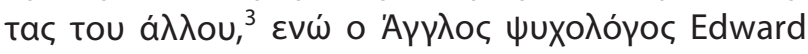

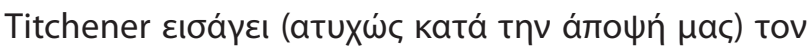

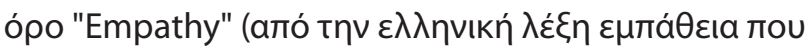

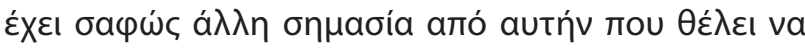

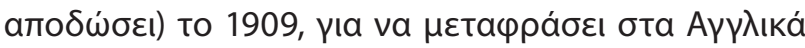

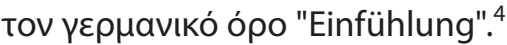

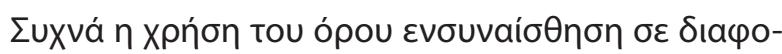

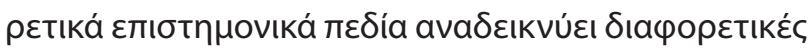

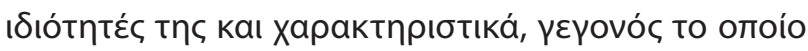

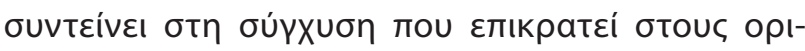

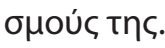

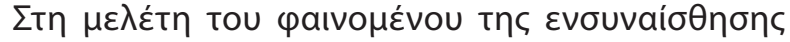

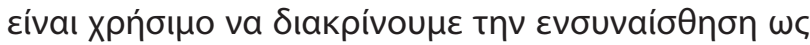

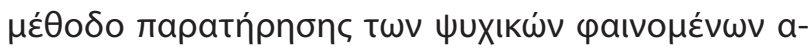

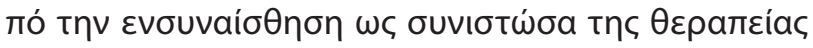

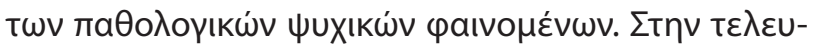

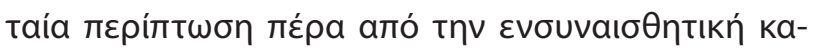

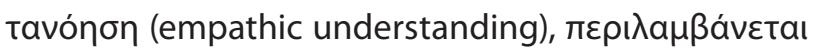

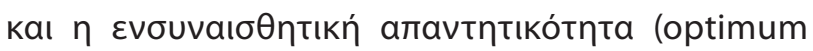

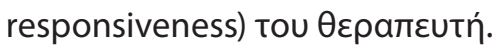

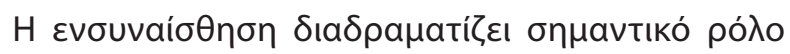

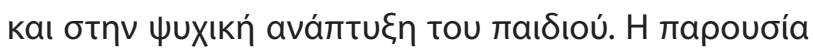

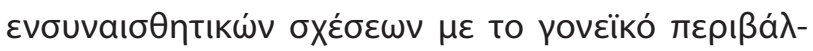

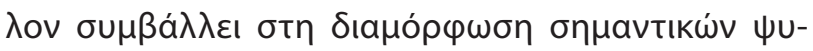

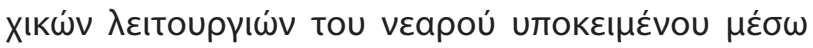

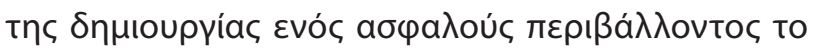

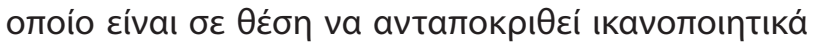

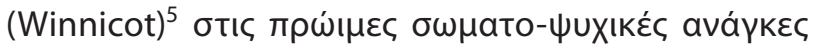

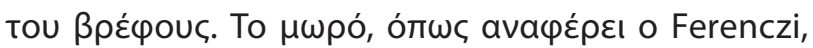

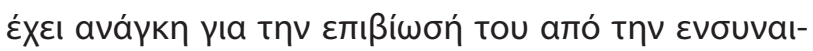

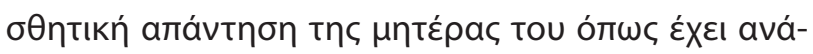

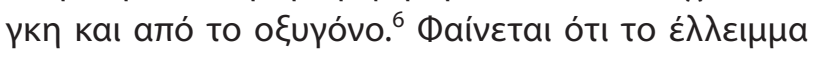

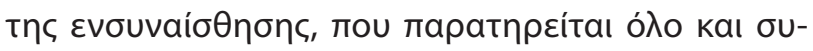

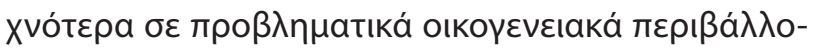

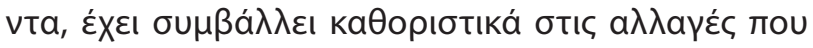

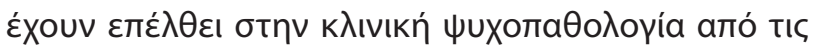

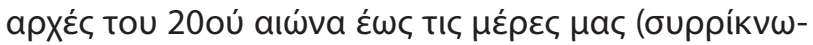

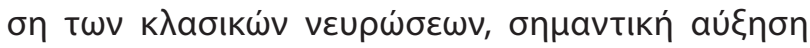

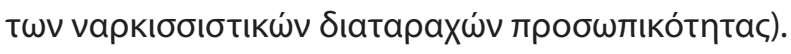

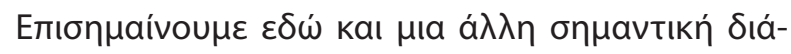

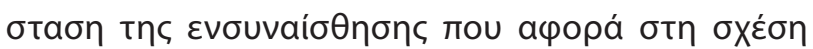

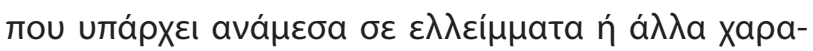

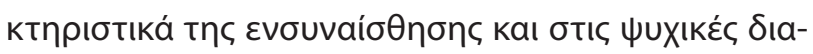

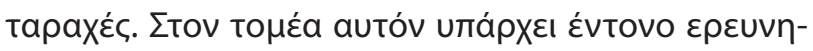

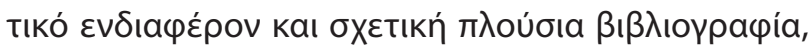

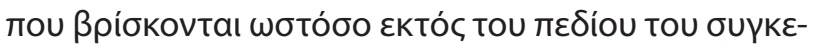

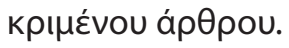




\section{Opı́pós}

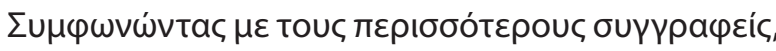

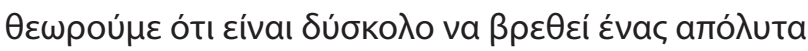

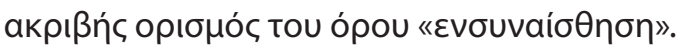

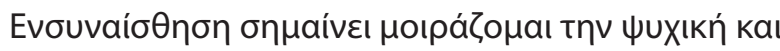

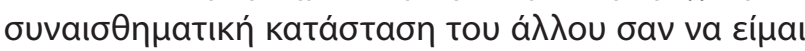

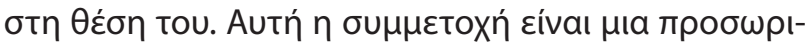

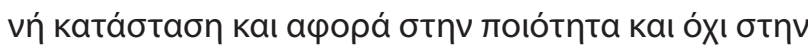

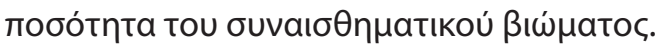

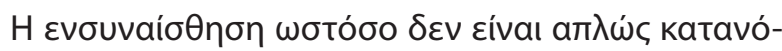

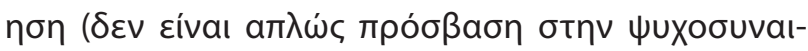

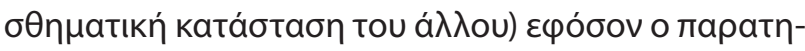

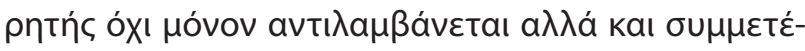

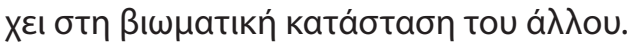

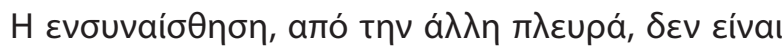

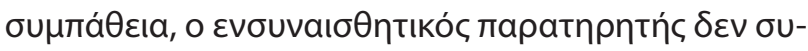

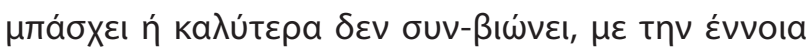

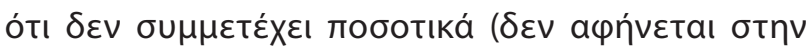

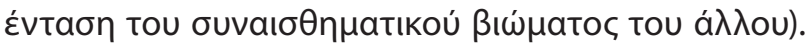

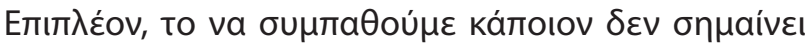

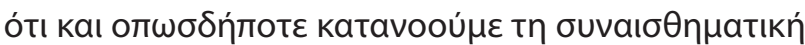
tou katáotaon.

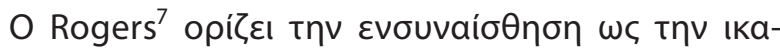

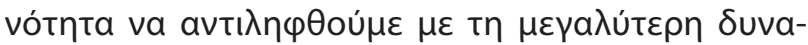

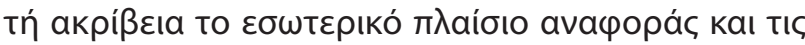

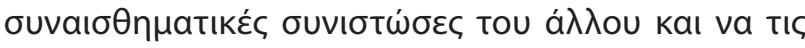

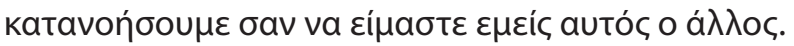

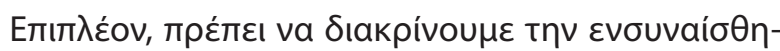

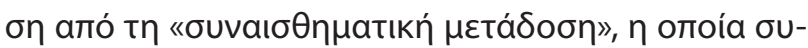

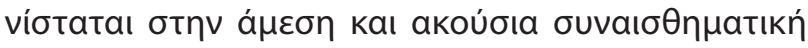

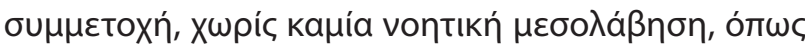

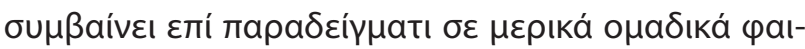
vó $\mu \varepsilon v a$.

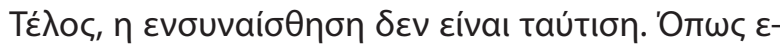

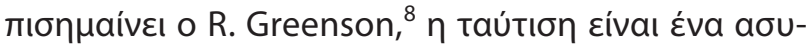

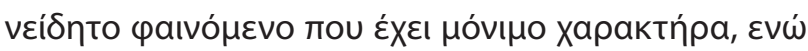

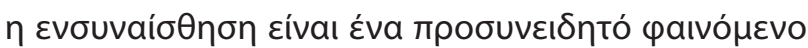

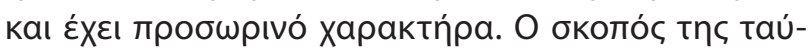

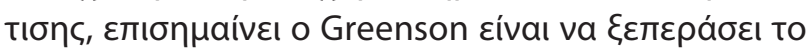

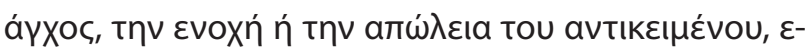

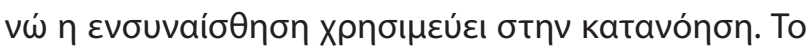

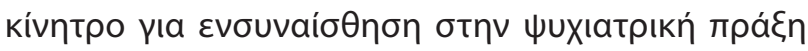

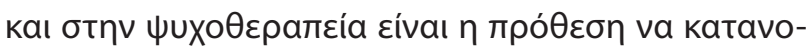

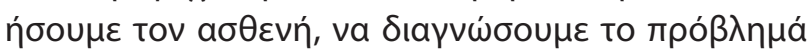

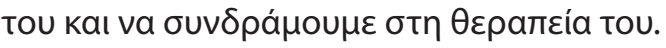

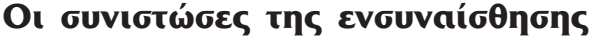

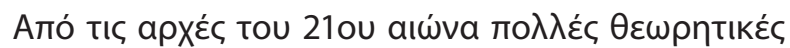

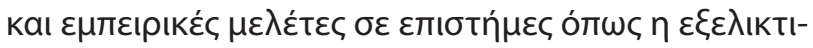

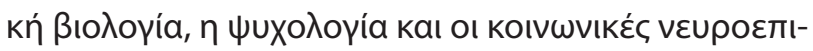

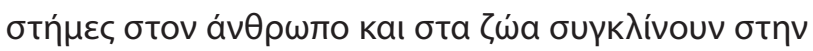

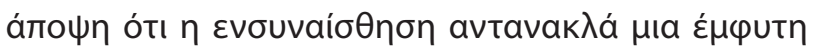

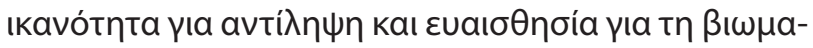

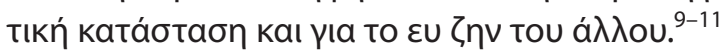

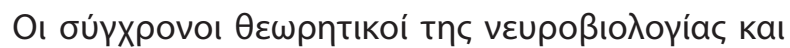

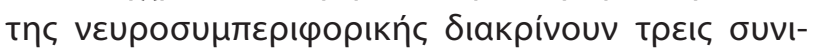

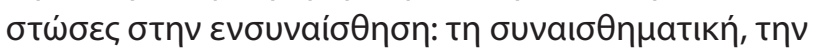

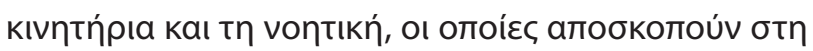

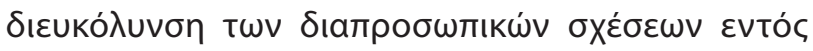

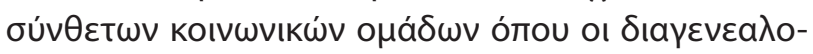

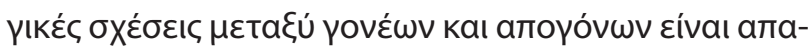

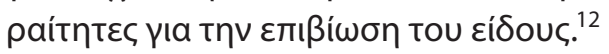

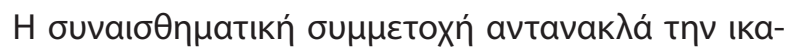

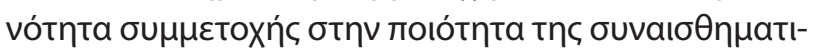

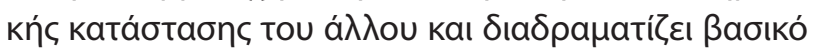

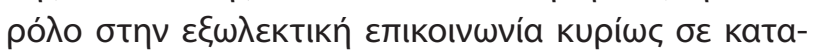

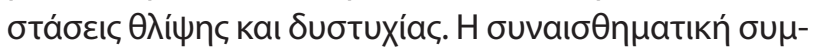

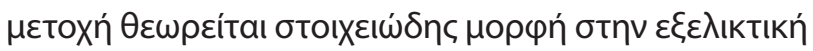

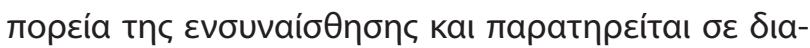

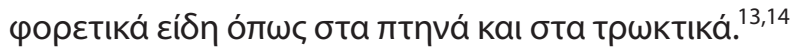

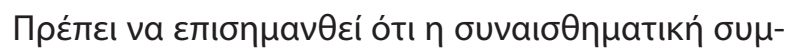

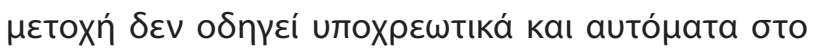

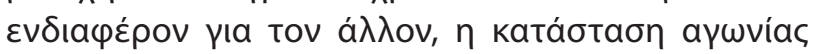

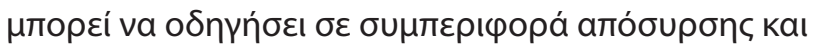

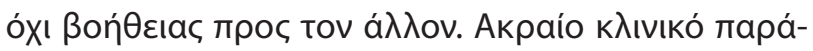

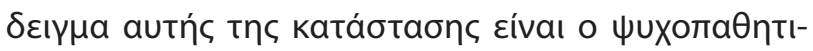

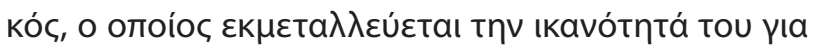

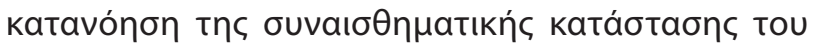

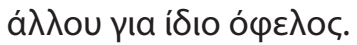

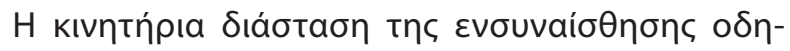

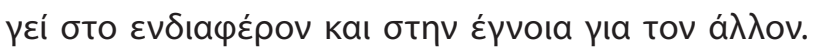

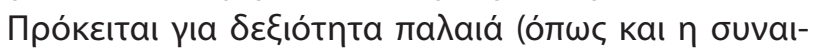

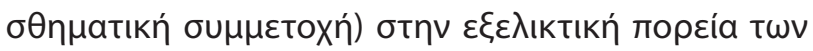

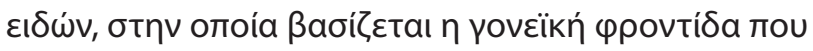

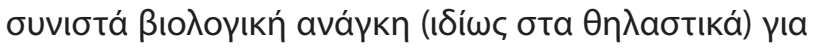

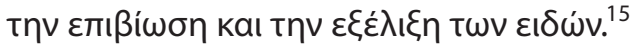

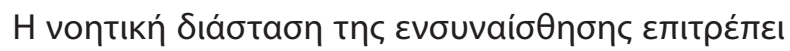

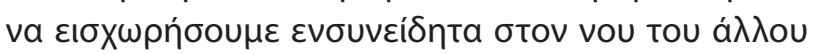

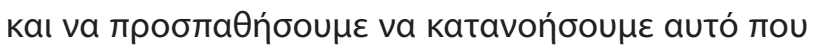

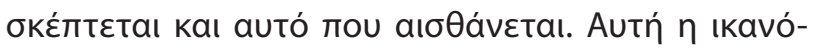

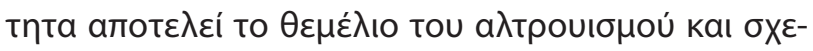

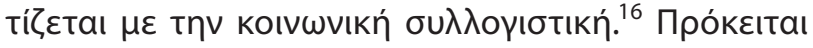




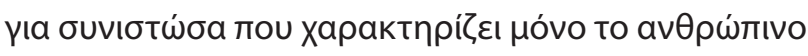

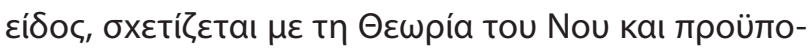

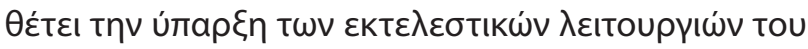

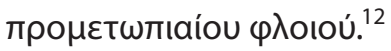

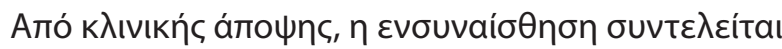

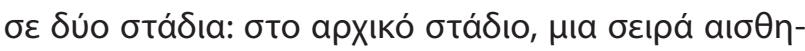
тпрıак

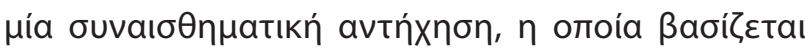

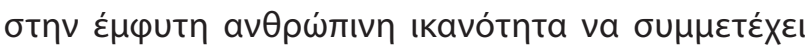

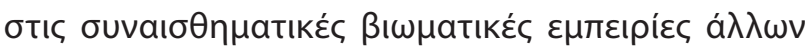

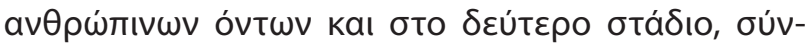

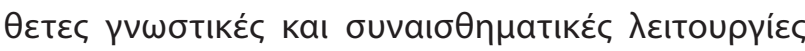

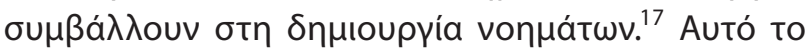

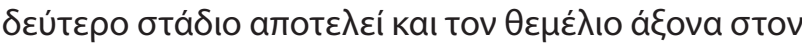

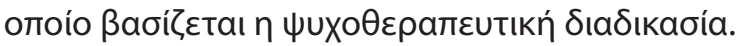

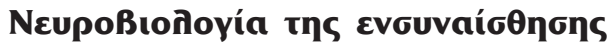

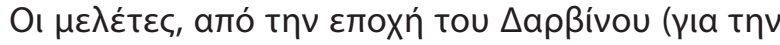

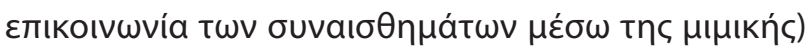

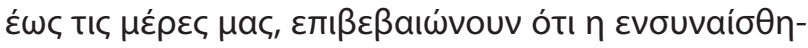

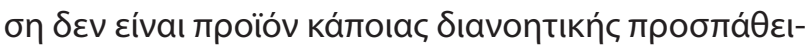
ac, a $\lambda \lambda$ á a

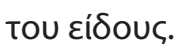

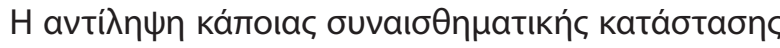

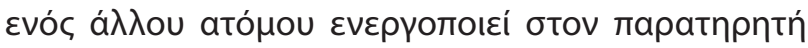

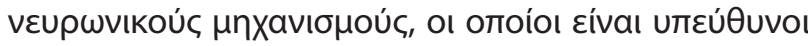

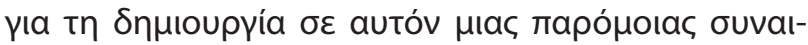

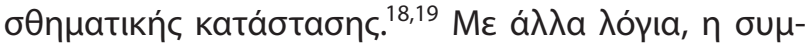

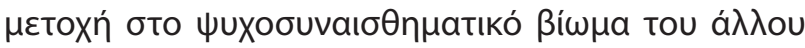

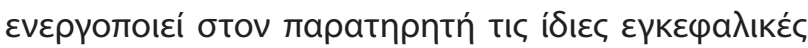

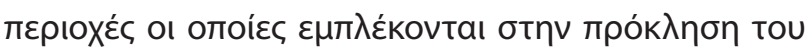

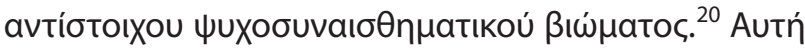

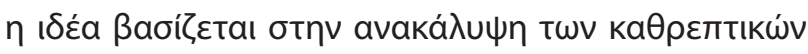

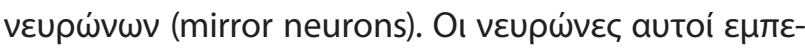

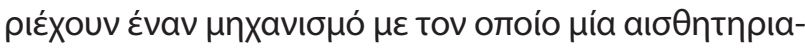

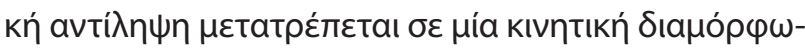

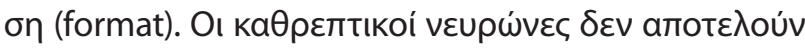
кámoı

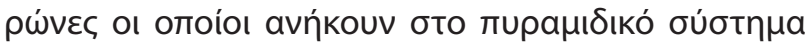

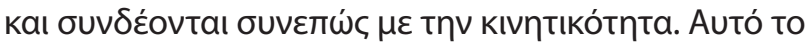

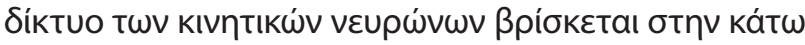

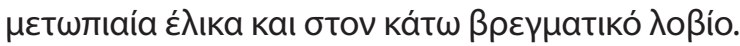

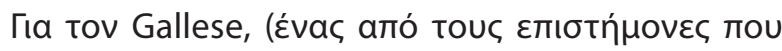

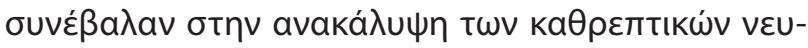

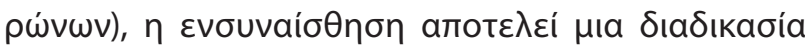

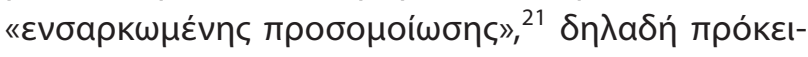

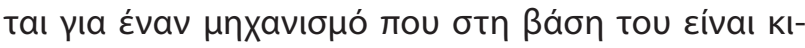

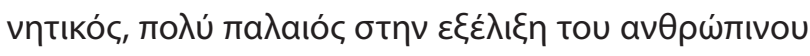

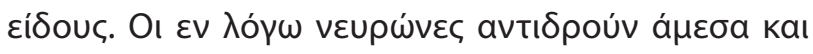

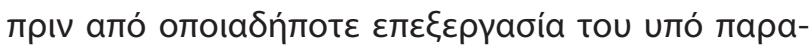

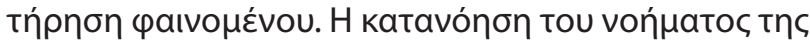

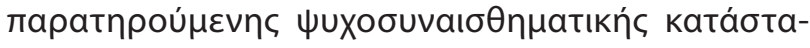

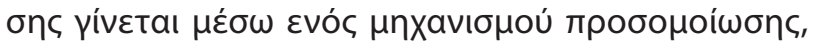

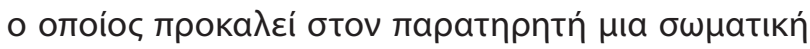

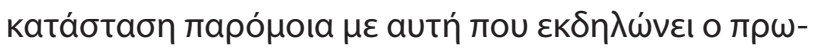

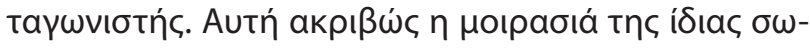

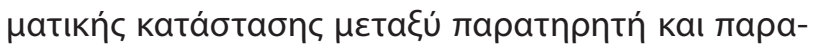

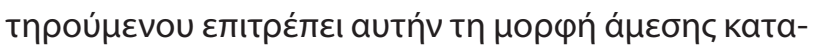

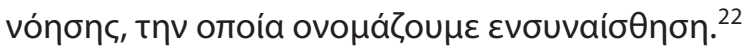

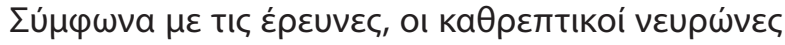

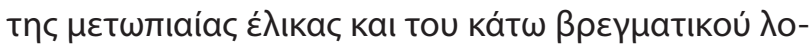

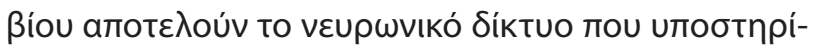

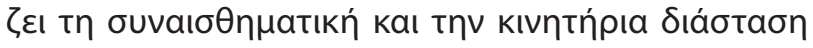

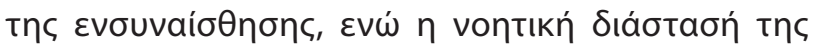

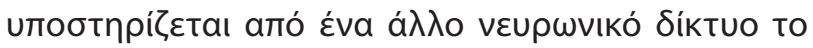

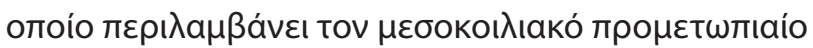

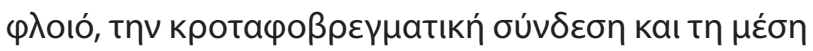

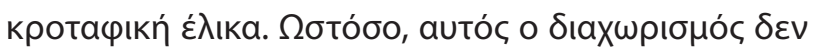

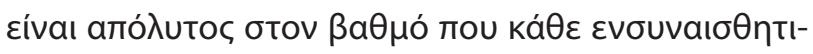

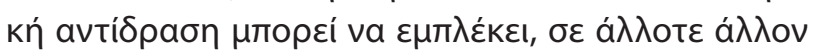

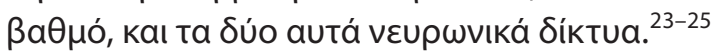

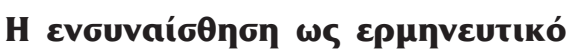

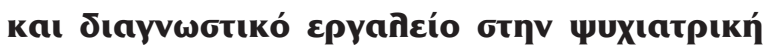

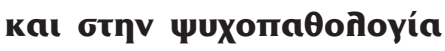

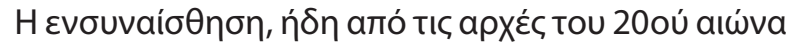

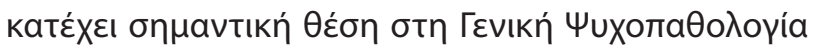

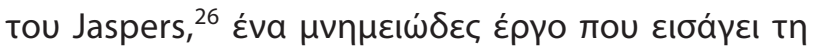

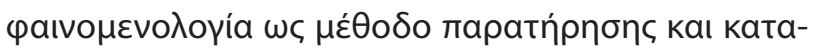

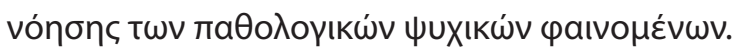

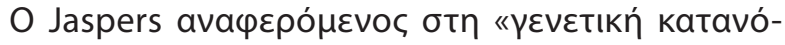

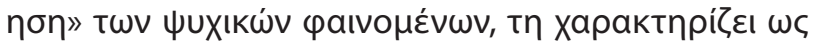

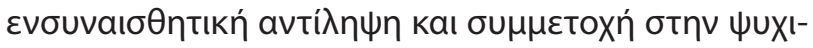

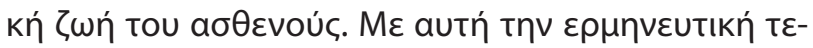

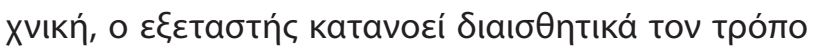

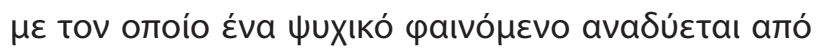

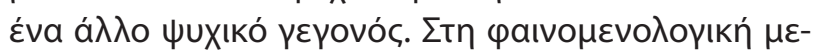

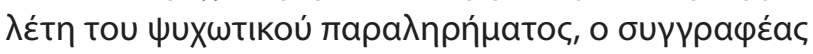

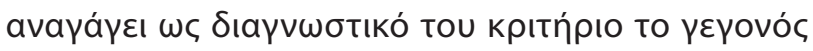

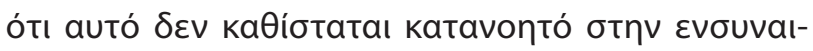

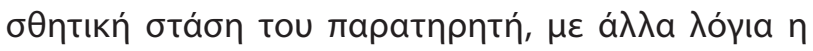

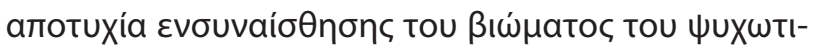

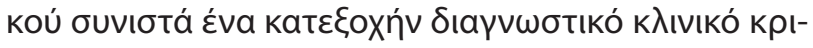

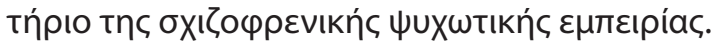




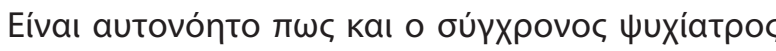

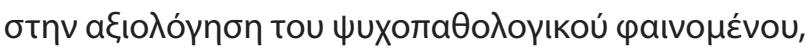

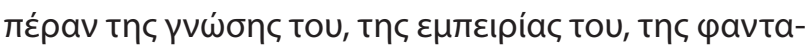

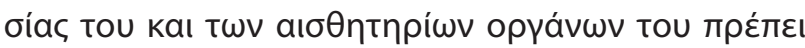

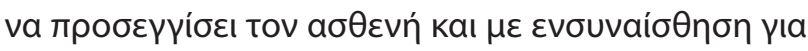

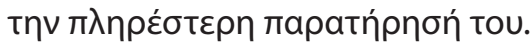

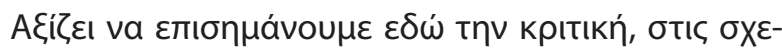

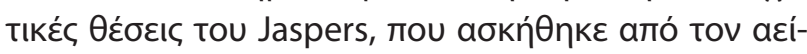

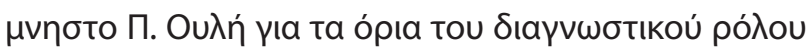

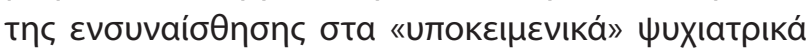

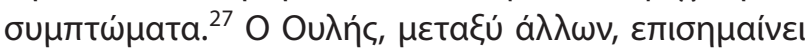

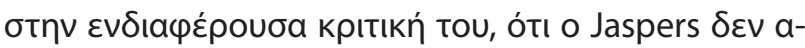

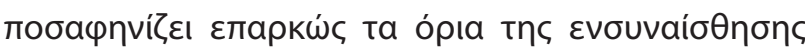

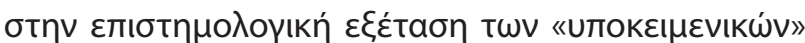

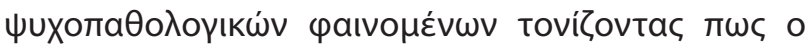

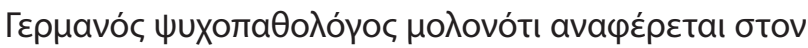

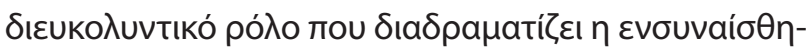

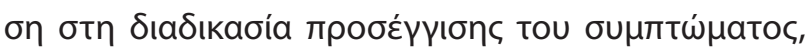

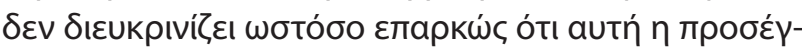

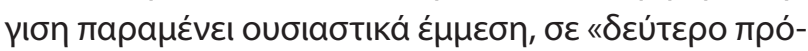

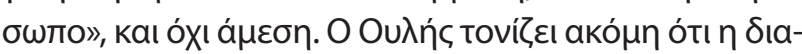

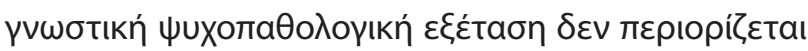

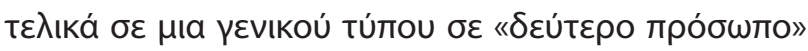

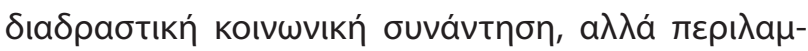

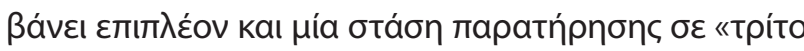

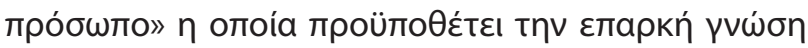

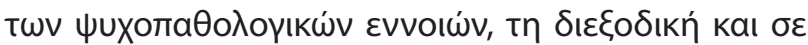

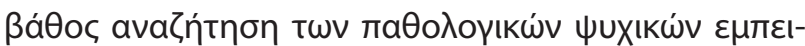

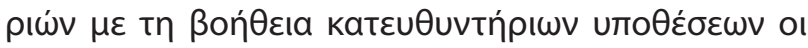

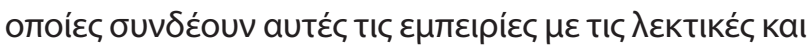

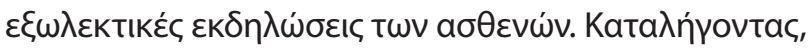

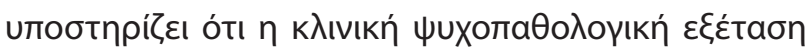

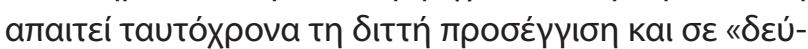

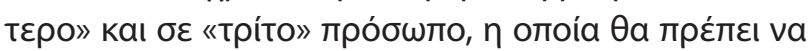

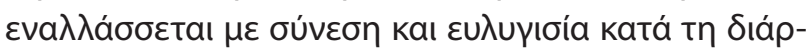

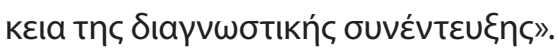

\section{H Évvola tns Evguvaíönons

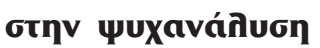

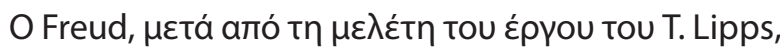

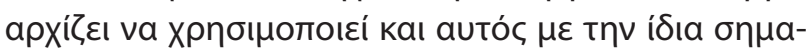

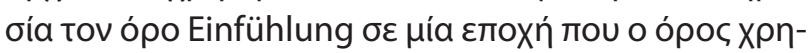

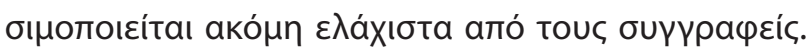

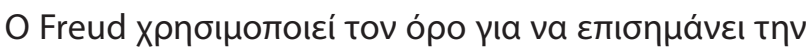

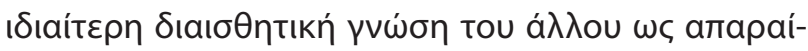

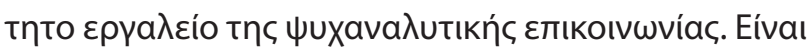

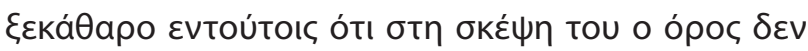

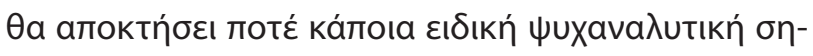

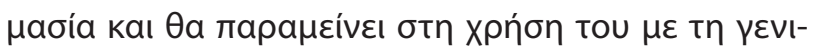

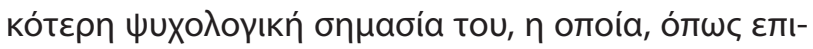

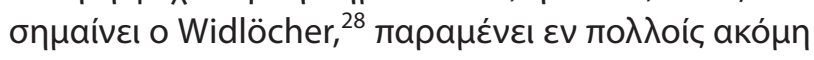

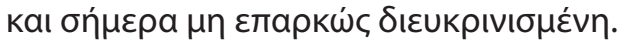

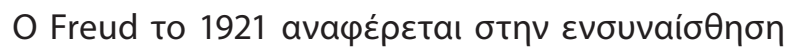

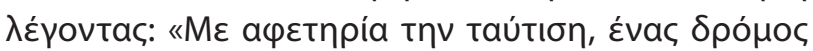

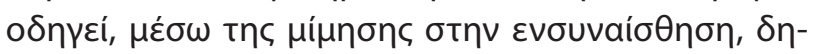

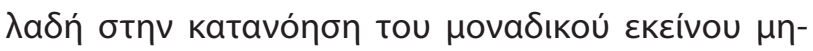

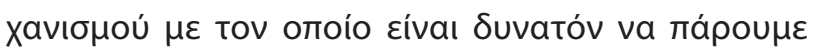

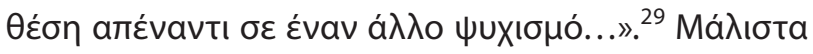

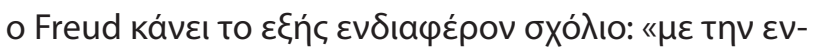

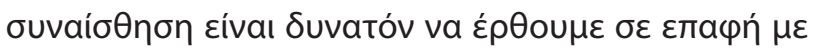

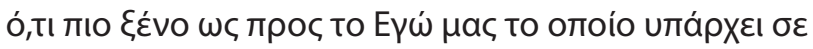

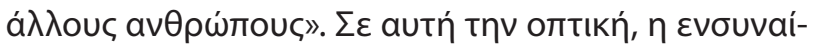

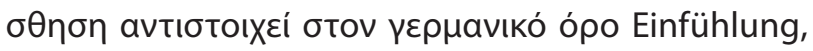

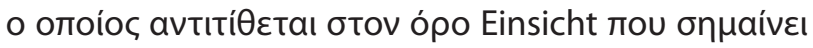

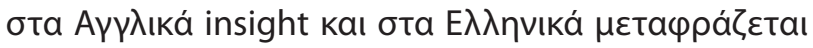

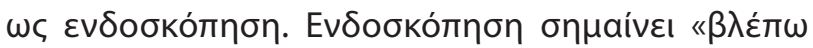

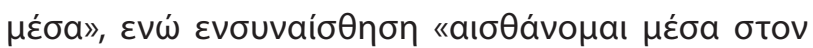

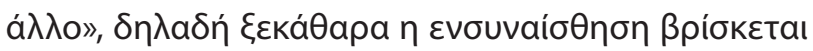

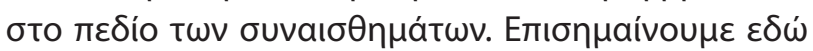

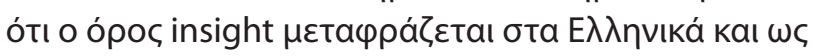

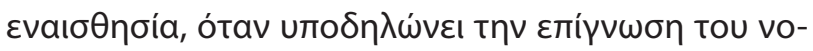

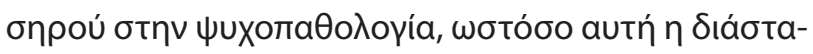

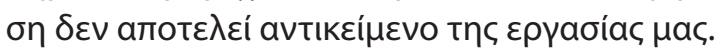

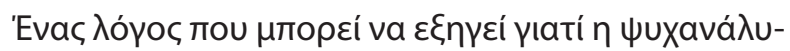

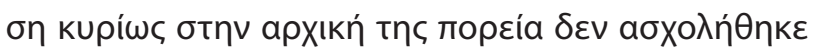

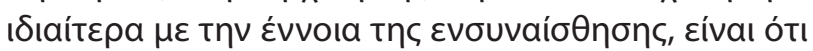

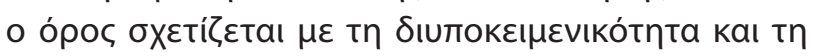

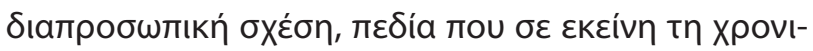

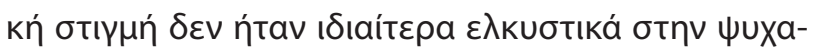

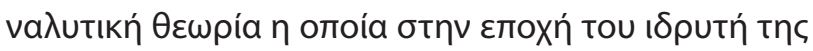

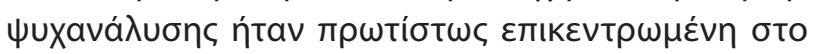

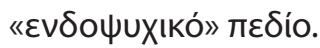

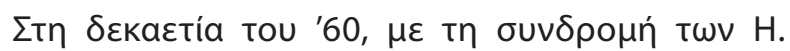

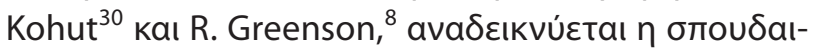

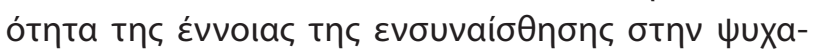

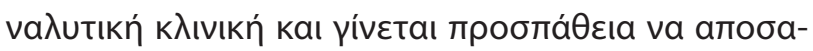

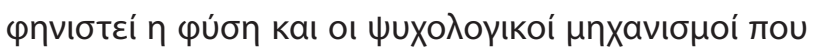

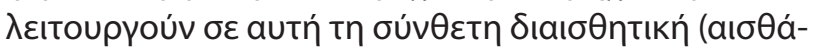

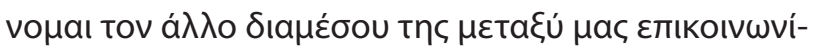

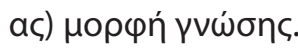

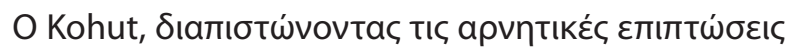

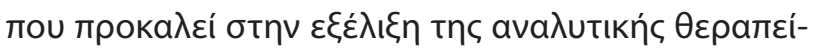

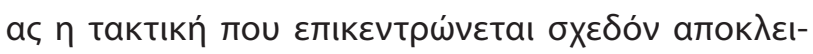

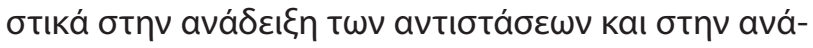


$\lambda$

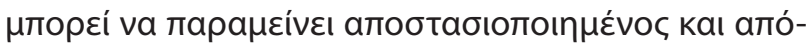

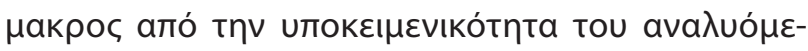

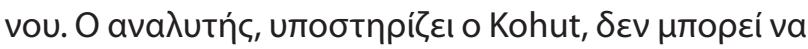

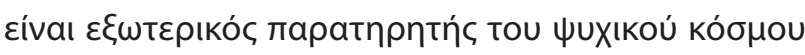

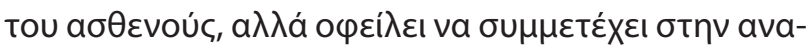

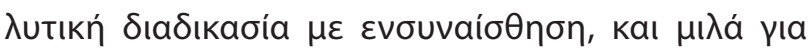

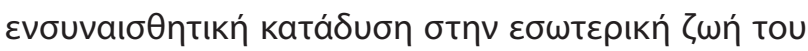

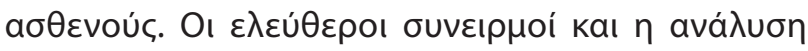

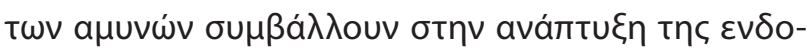

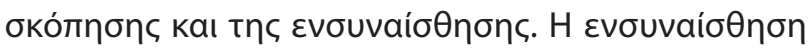

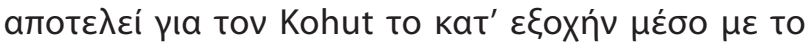

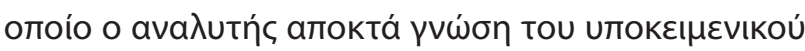

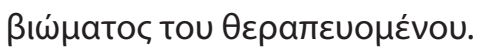

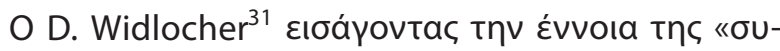

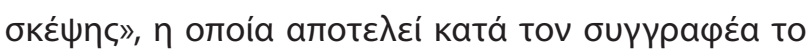

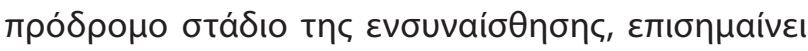

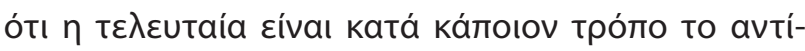

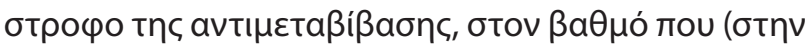

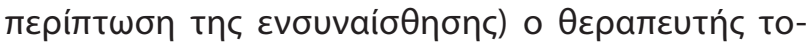

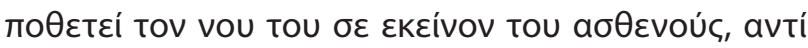

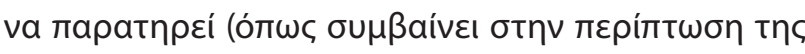

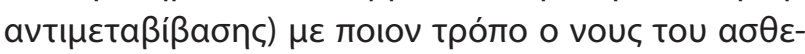

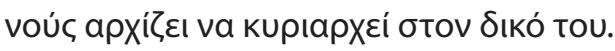

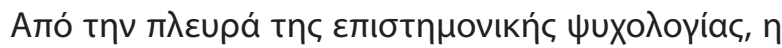

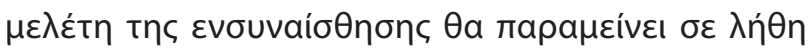

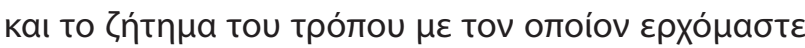

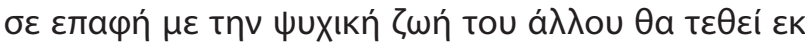

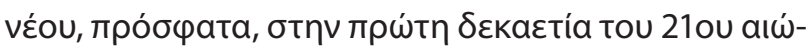

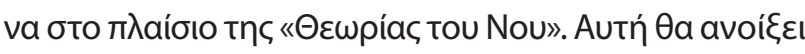

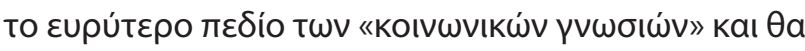

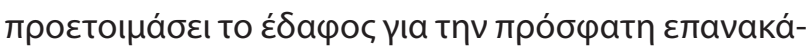

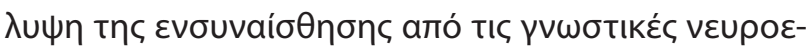

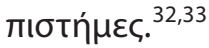

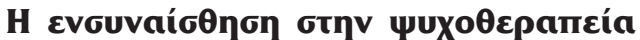

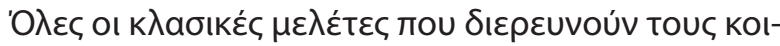

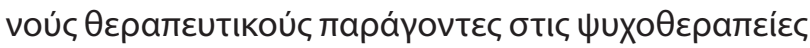

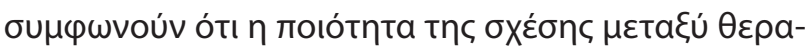

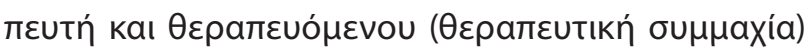

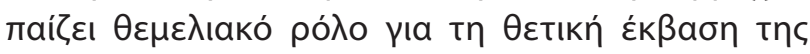

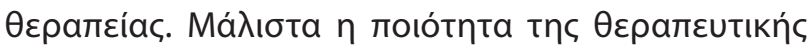

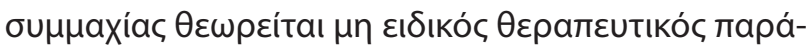

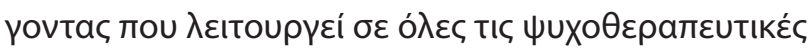

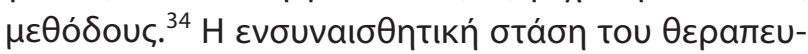

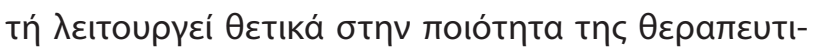

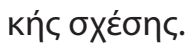

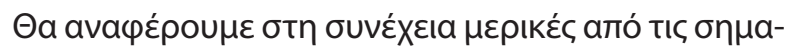

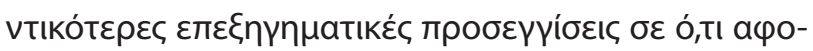

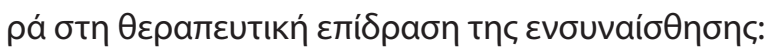

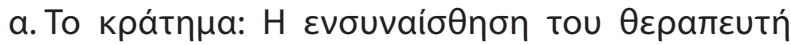

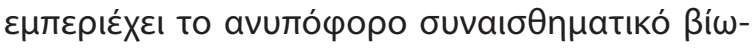

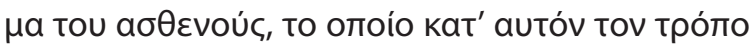
каӨі́бтатаı пı итоферто́. ${ }^{35}$

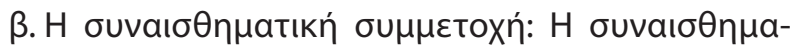

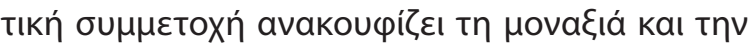

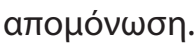

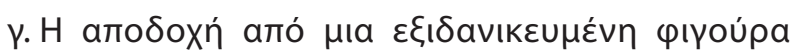

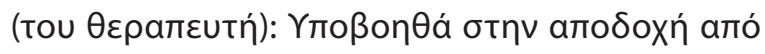

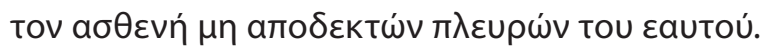

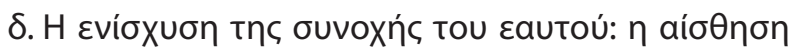

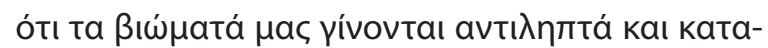

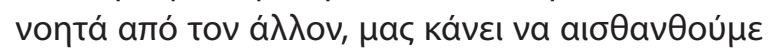

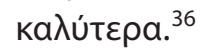

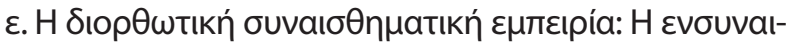

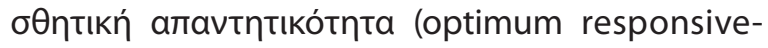

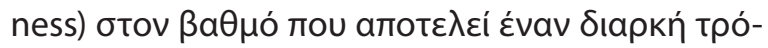

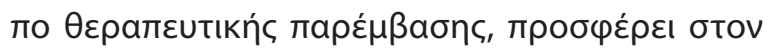

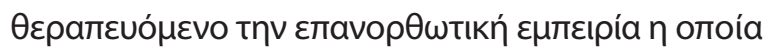

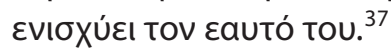

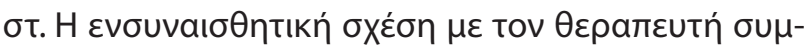

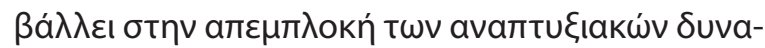

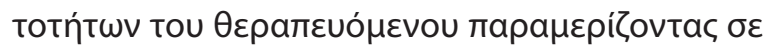

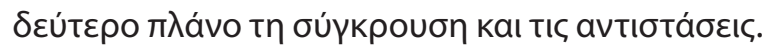

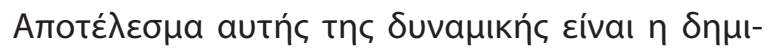

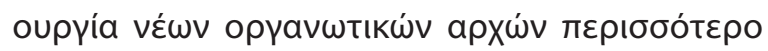

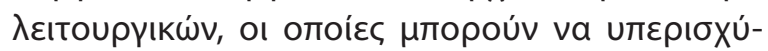

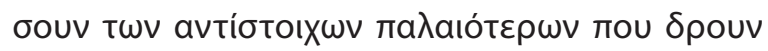

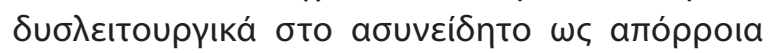

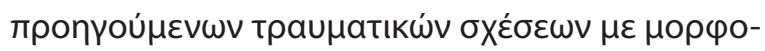

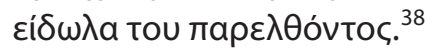

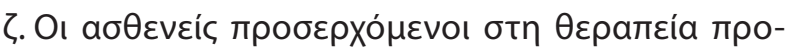

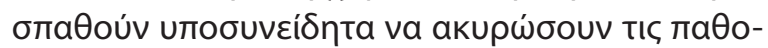

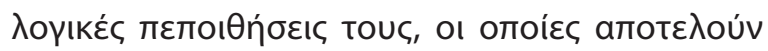

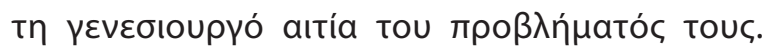

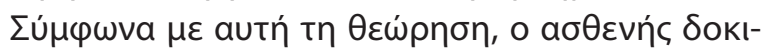

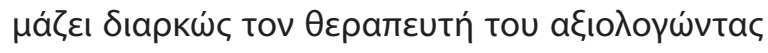

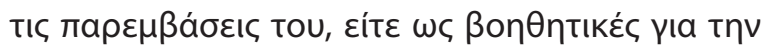

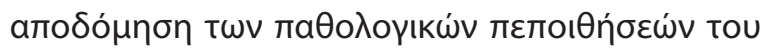

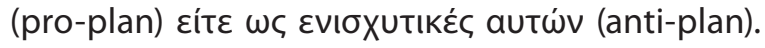

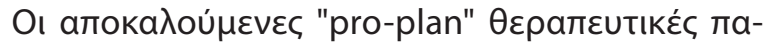




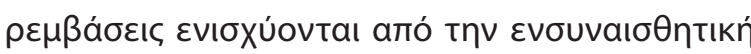
otáon tou $\theta \varepsilon \rho a \pi \varepsilon u t$. $^{39}$

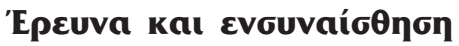

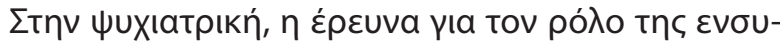

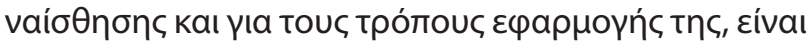

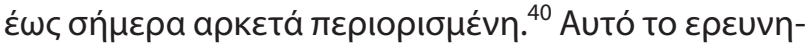

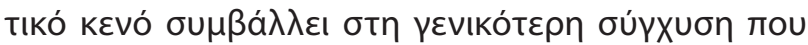

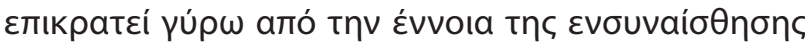

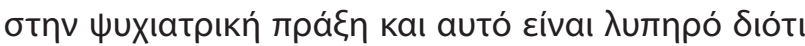

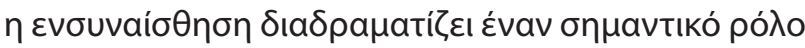

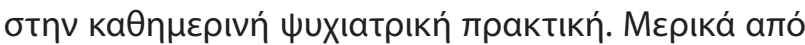

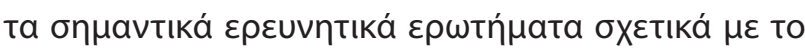

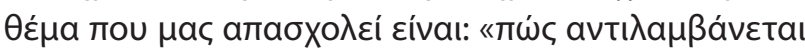

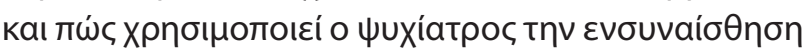

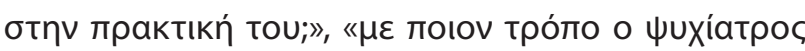

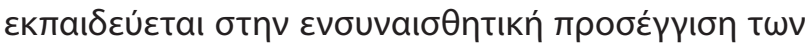

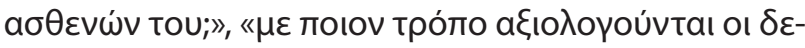

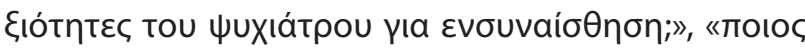

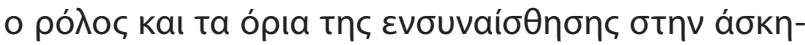

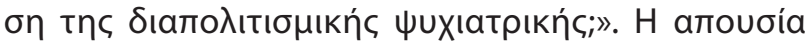

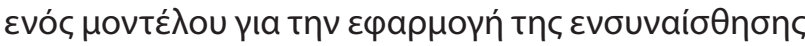

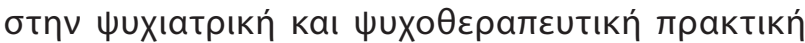

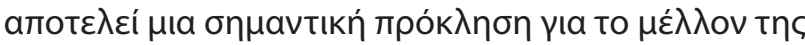

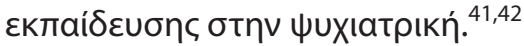

\section{Emínoyos}

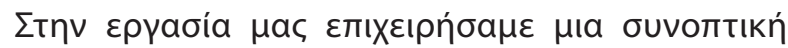

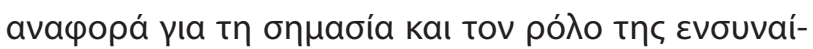

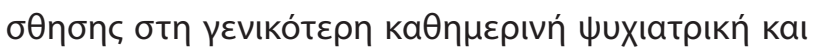

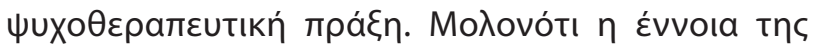

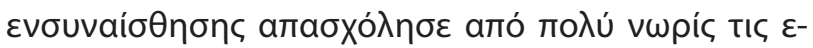

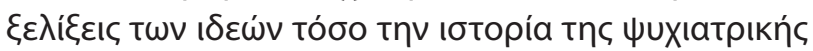

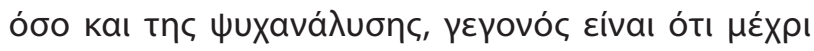

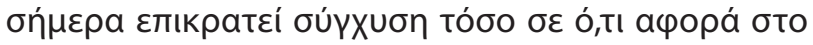

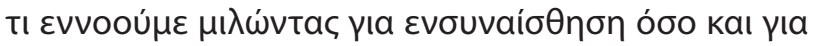

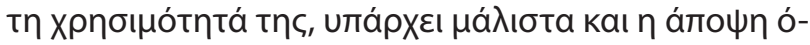

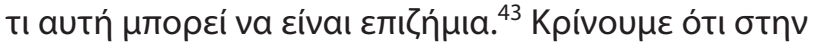

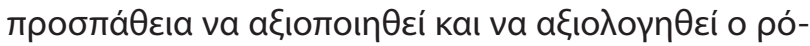

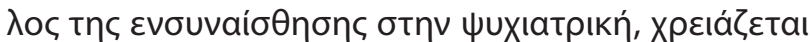

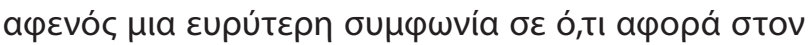

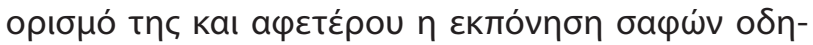

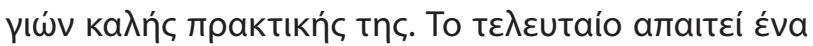

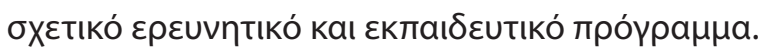

\section{Euxapiotícs}

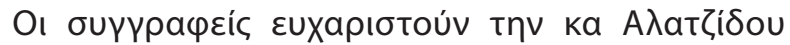

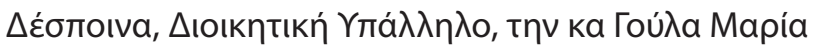

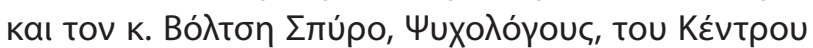

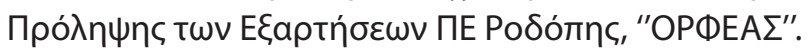

\title{
Empathy in psychiatry and psychotherapy
}

\author{
G. Esagian, ${ }^{1}$ S. Esagian-Pouftsis, ${ }^{2}$ S.G. Kaprinis ${ }^{3}$ \\ ${ }^{1}$ Addiction Prevention and Psychosocial Health Promotion Center, ORPHEAS, Rodopi, Komotini, Greece, \\ ${ }^{2}$ Center for Brief Therapy, Nant Foundation, Vaud East Psychiatric Institute, Montreux, Switzerland, \\ ${ }^{3} 2$ nd Department of Psychiatry, Medical School, Aristotle University of Thessaloniki, Greece
}

Psychiatriki 2019, 30:156-164

Empathy is the epicenter of the theoretical and clinical interest of many different scientific domains, constituting a common term in different fields as these of psychology, psychiatry, clinical psychopathology, neuroscience, psychotherapy and psychoanalysis. Studying the phenomenon of empathy requires distinguishing the empathy as a method of observation of psychological phenomena from the empathy as a component of the therapy of psychopathological phenomena. In this case, beyond the empathic understanding, the optimum responsiveness of the therapist is included. Empathy means sharing the psychological and emotional state of another as if you were able to sense one's private world. This emotional sharing is a temporary situation and concerns the quality but not the quantity of the emotional experience. The modern scientists of neurobiology and neurobehavior distinguish three types of empathy: the emotional, the cognitive and motivational which help the 
interpersonal relations occurring in complex groups of society where the transgenerational relationships between parents and offspring are essential for the survival of species. Jaspers introduces, already by the beginning of the 20th century in his monumental work "General Psychopathology", the notion of empathy as an interpretative and a diagnostic tool in psychiatry and psychopathology. Meanwhile psychoanalysis, mainly during its early course, was not impressed by this term. The answer to this seems to be that the term empathy is related to the intersubjectivity and the interpersonal relationship, fields that were not really attractive at this point of time for the psychoanalytical theory which during the period of the founder of the psychoanalysis was initially mainly focused on the "intrapsychic" field. However, during the course of time and chiefly with the contribution of Kohut and Greenson, the importance of the concept of empathy is emphasized in the clinical psychoanalysis and efforts are made regarding the clarification of the nature and the psychological mechanisms that operate in this complex intuitive (feeling the other through the interpersonal communication) form of knowledge. The quality of the relationship between the therapist and the patient (therapeutic alliance) plays a fundamental role in the positive outcome in any form of psychotherapy. Empathic understanding plays an important role in the quality of the therapeutic relationship. In the field of general psychiatry, the focus of research for the role of empathy and the potential of its application, has been until today limited. The lack of a model for the application of empathy in psychiatric and psychotherapeutic practice is an important challenge for the training of the new psychiatrists.

Key words: Empathy, psychiatric practice, therapeutic alliance, psychotherapy.

\section{BıßAtoypacpía}

1. Batson CD. These things called empathy: Eight related but distinct phenomena. In: Decety J, Ickes W (eds) The Social Neuroscience of Empathy. MIT Press, Massachusetts, 2009

2. Vischer R. Ueber das optische Formgefühl: Ein Beitragzur Ästhetik. Credner H, Leipzig, 1873

3. Lipps T. Psychologische Untersuchungen. Verlag Von Wilhelm Engelmann, Leipzig, 1907

4. Titchener EB. The Psychology of Feeling and Attention. J Philosoph Psychol Scientif Method 1909, 6:64-77, doi: 10.2307/ 2010623

5. Winnicott DW. The Child, the Family and the Outside World. Pelican Books, Middlesex, 1969

6. Ferenczi S. Confusion of the Tongues Between the Adults and the Child - The Language of Tenderness and of Passion. Contempor Psychoanal 2013, 24:2, 196-206, doi: 10.1080/ 00107530.1988 .10746234

7. Rogers CR. Client-centered therapy. In: Arieti S (ed) American handbook of psychiatry. Vol 3. Basic Books, New York, 1966

8. Greenson RR. Empathy and its vicissitudes. Int J Psychoanal 1960, 41:418-424

9. Decety J. The neuroevolution of empathy. Annals of the New York Academy of Sciences Trans NY Acad Sci 2011, 1231: 35-45, doi: 10.1111/j.1749-6632.2011.06027.x

10. Decety J, Jackson PL. The functional architecture of human empathy. Behav Cogn Neurosci Rev 2004, 3:71-100, doi: 10.1177/ 1534582304267187

11. Decety J, Svetlova M. Putting together phylogenetic and ontogenetic perspectives on empathy. Behav Cogn Neurosci Rev 2002, 2:1-24, doi: 10.1016/j.dcn.2011.05.003
12. Decety J. Composants, mécanismes, développement et fonctions de l' empathie. EMC-Psychiatrie 2015, 13:1-8, doi: 10.1016/S0246-1072(15)70299-3

13. Bartal B, Decety J, Mason P. Empathy and pro-social behavior in rats. Science. 2011, 334:1427-1430, doi: 10.1126/science. 1210789

14. Edgar JL, Lowe JC, Paul ES, Nicol CJ. Avian maternal response to chick distress. Proc R Soc Lond B Biol Sci 2011, 278:31293134, doi: 10.1098/rspb.2010.2701

15. Decety J, Norman GJ, Berntson GG, Cacioppo JT. A neurobehavioral evolutionary perspective on the mechanisms underlying empathy. Prog Neurobiol 2012, 98:38-48, doi: 10.1016/j. pneurobio.2012.05.001

16. Underwood B, Moore B. Perspective-taking and altruism. Psychol Bull 1982, 91:143-173, doi: 10.1037/0033-2909.91.1.143

17. Feiner K, Kiersky S. Empathy: A common ground. Psychoanal Dialog 1994, 4:425-440, doi.org/10.1177/0003065108322097

18. Rizzolatti G, Craighero L. The mirror-neurons system. Ann Rev Neurosci 2004, 27:169-192, doi: 10.1146/annurev.neuro.27. 070203.144230

19. Preston SD, de Waal FB. Empathy: Its ultimate and proximate bases. Behav Brain Sci 2002, 25:1-72, PMID: 12625087

20. Gallese V. "Being Like Me": Self-Other Identity, Mirror Neurons, and Empathy. In: Hurley S, Chater N (eds) Perspectives on imitation: From neuroscience to social science. Vol. 1. Mechanisms of imitation and imitation in animals. MIT Press, Massachusetts, 2005

21. Gallese V. Embodied simulation: from neurons to phenomenal experience. Phenomenol Cognitiv Sci 2005, 4:23-48, doi: $10.1007 /$ s 11097-005-4737-z 
22. Gallese V, Eagle MN, Migone P. Intentional attunement: mirror neurons and the neural underpinnings of interpersonal relations. J Am Psychoanal Assoc 2007, 55:131-176, doi: 10.1177/ 00030651070550010601

23. Shamay-Tsoory S. The neuropsychology of empathy: evidence from lesion studies. Rev Neuropsychol 2015, 7:237-243 doi: 10.1684/nrp.2015.0356

24. Bernhardt BC, Singer T. The Neural Basis of Empathy. Ann Rev Neurosci 2012, 35:1-23, doi: 10.1146/annurev-neuro-062111150536

25. Zaki J, Ochsner K. The neuroscience of empathy: progress, pitfalls and promise. Nat Neurosci 2012, 15, 5:675-680, doi: 10.1038/nn.3085

26. Jaspers K. Allgemeine Psychopathologie, einLeitfadenfürStudierende, ÄrzteundPsychologen. Springer, Berlin, 1913

27. Oulis $\mathrm{P}$. The epistemological role of empathy in psychopathological diagnosis: a contemporary reassessment of Karl Jaspers' account. Philosoph Ethic Human Med 2014, 9:6, doi. org/10.1186/1747-5341-9-6

28. Widlöcher D. Dissection de l' empathie. Rev Fr Psychanal 2004, 68:981-992, doi: 10.3917/rfp.683.0981

29. Freud S. Massenpsychologieundlch-Analyse. Internationaler Psychoanalytischer Verlag, Vienna, 1921

30. Kohut H. Introspection, empathy, and psychoanalysis: an examination of the relationship between mode of observation and theory. J Am Psychoanal Assoc 1959, 7:459-483, doi: 10.1177/000306515900700304

31. Widlöcher D. Affect et empathie. Rev Fr Psychanal 1999, 63:173188, doi: 10.3917/rfp.g1999.63n1.0173

32. Decety J. L' empathie est-elle une simulation mentale de la subjectivitı d'autrui? In: Berthoz A, Jorland G (eds) L'Empathie. Odile Jacob, Paris, 2004

33. Georgieff N. Psychanalyse et neurosciences du lien: nouvelles conditions pour une rencontre entre psychanalyse et neurosciences. Rev Fr Psychanal 2007, 71:501-516, doi: 10.3917/rfp. 712.0501
34. Norcross JC. Psychotherapy Relationships That Work: Therapist Contributions and Responsiveness to Patients. Oxford University Press, New York, 2002

35. Winnicott DW. Dependence in infant care, child care, psychoanalytic setting. Intern J Psychoanal 1963, 44:339-344, PMID: 14001207

36. Wolf ES. Treating the Self: Elements of clinical self psychology. The Guilford Press, New York, 2002

37. Bacal HA. The elements of a corrective self object experience. Psychoanal Inquir 1990, 10:347-372, doi: 10.1080/ 07351690.1990.10399611

38. Stolorow RD, Atwood GE. Contexts of being: The intersubjective foundations of psychological life (Psychoanalytic inquiry book series vol. 12). Analytic Press, New York, 2002

39. Weiss J, Sampson H. The Psychoanalytic Process: Theory, Clinical Observation, and Empirical Research. 1st ed. Guilford Press, New York, 1986

40. Ross J, Watling C. Use of empathy in psychiatric practice: constructivist grounded theory study. Br J Psychiatr Open 2017, 3:26-33, doi: 10.1192/bjpo.bp.116.004242

41. Bracken P, Thomas P, Timimi S, Asen E, Behr G, Beuster C et al. Psychiatry beyond the current paradigm. Br J Psychiatry 2012, 201:430-434, doi: 10.1192/bjp.bp.112.109447

42. Kleinman A. Rebalancing academic psychiatry: why it needs to happen - and soon. Br J Psychiatry 2012, 201:421-422, doi: 10.1192/bjp.bp.112.118695

43. Bloom P. Against empathy: The case for rational compassion. Ecco, New York, 2016

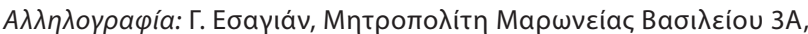

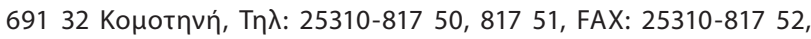
e-mail: kporfeas@otenet.gr 


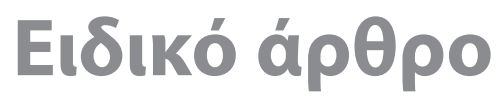 Special article
}

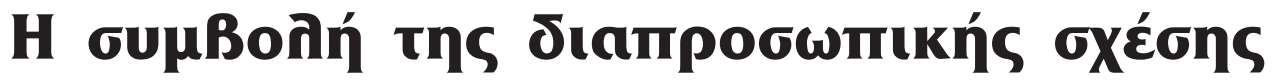

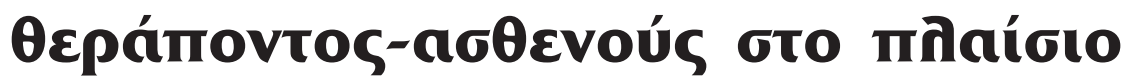

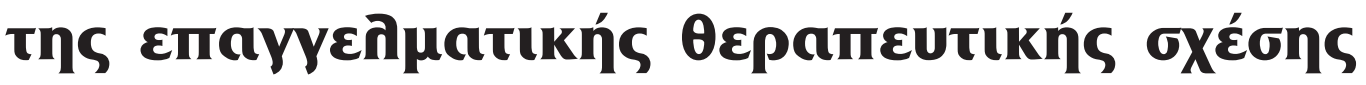

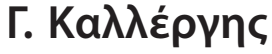

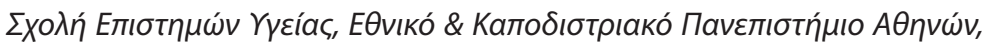

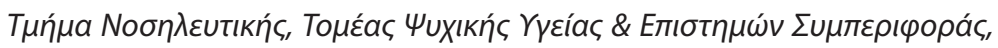

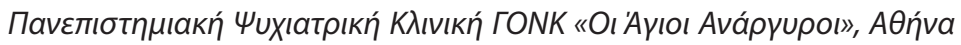

uuxıатрıкń 2019, 30:165-174

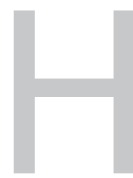

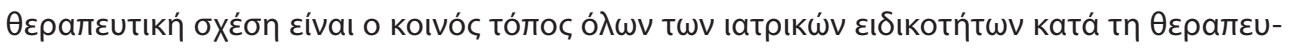

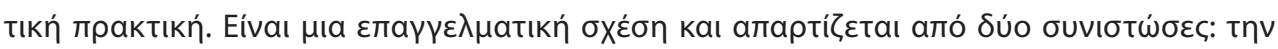

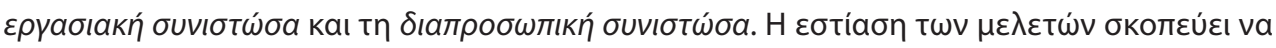

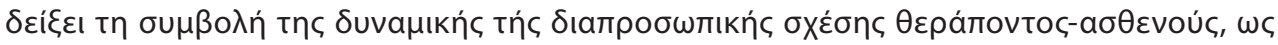

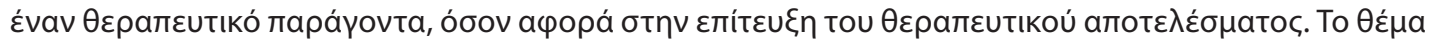

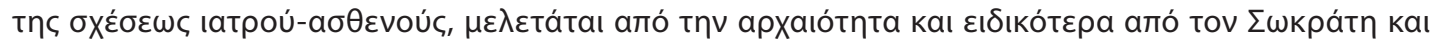

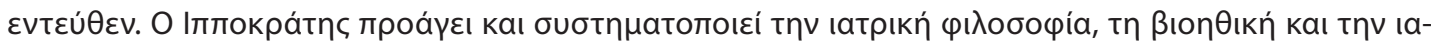

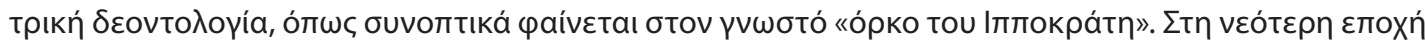

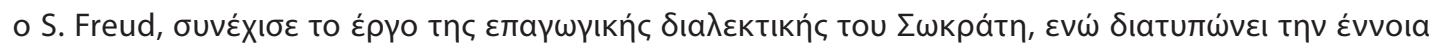

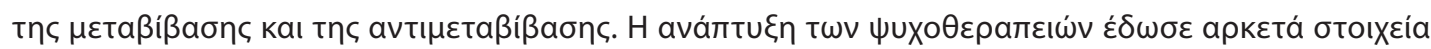

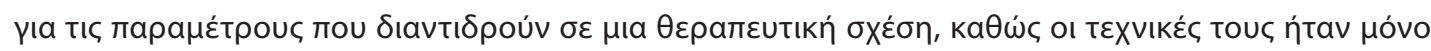

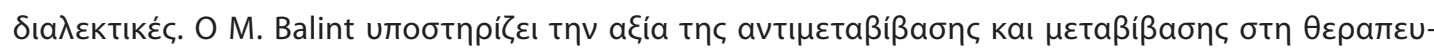

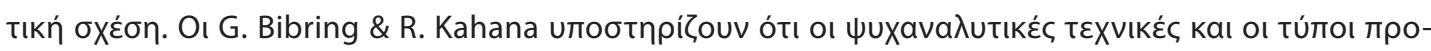

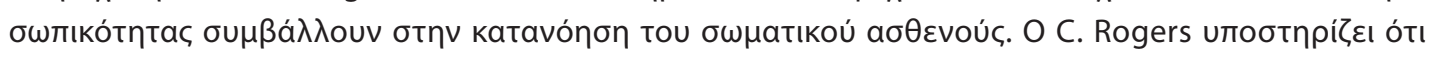

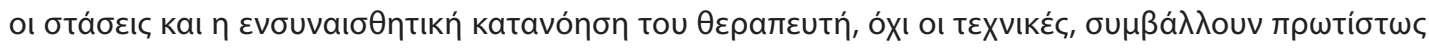

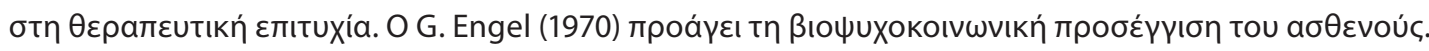

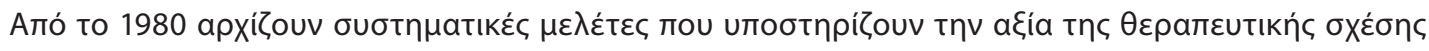

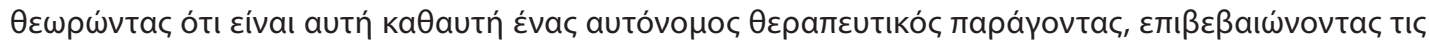

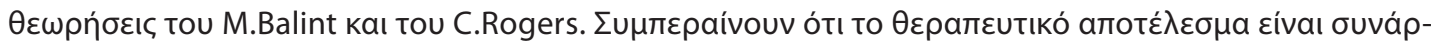




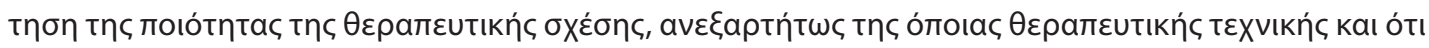
$\eta$ Ө

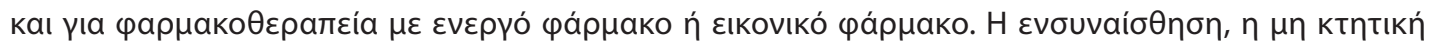

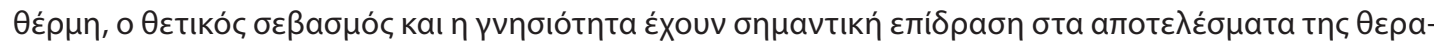

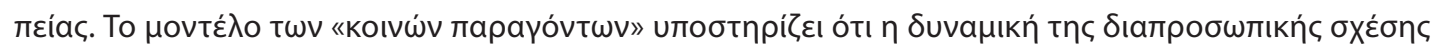

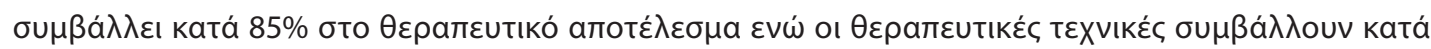

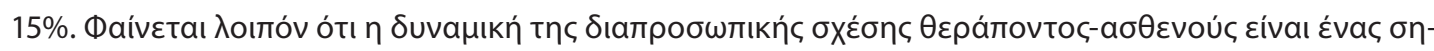

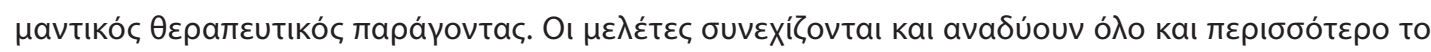

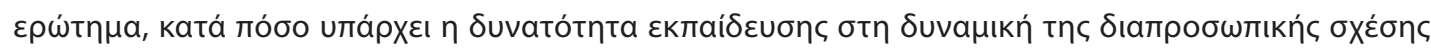

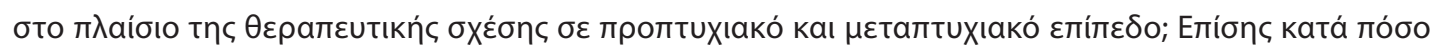

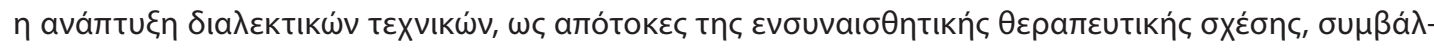

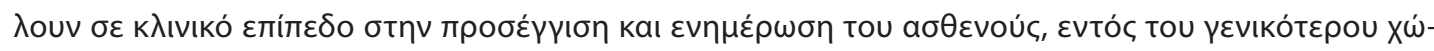

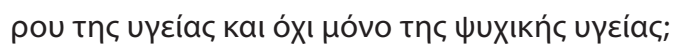

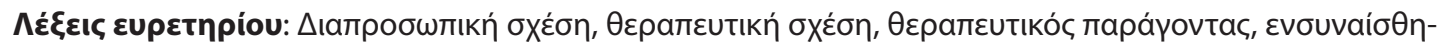
оп, avтıнстаßißaon.

\section{Eıбaywyń}

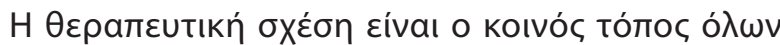

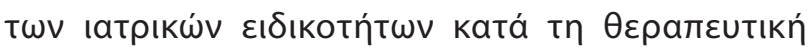

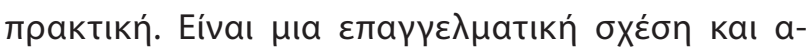

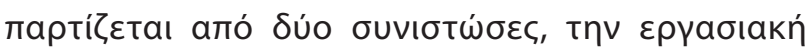

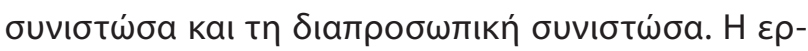

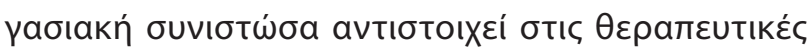

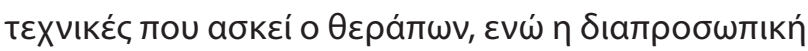

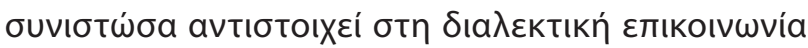

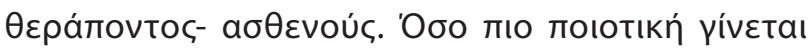

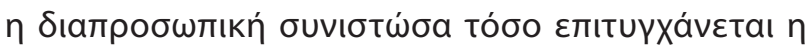

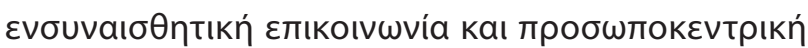

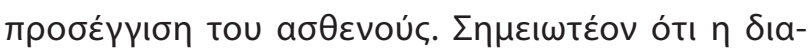

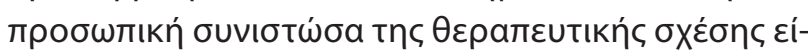

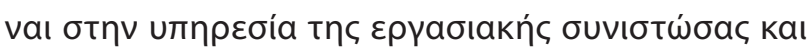

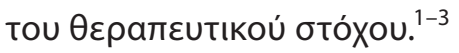

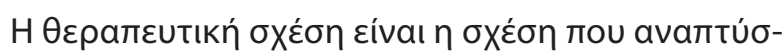

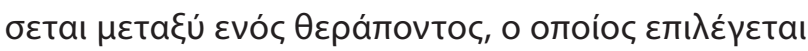

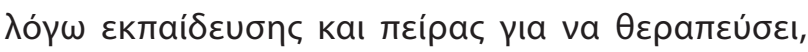

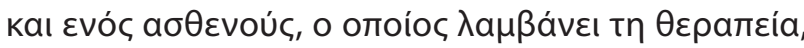

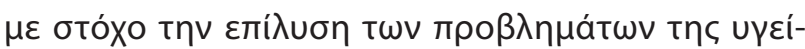

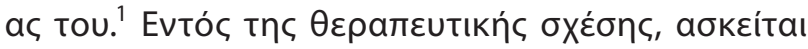

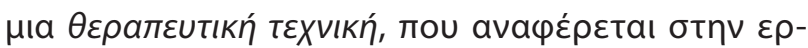

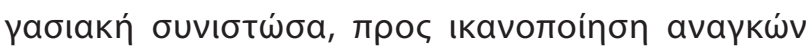

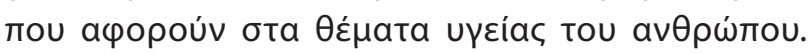

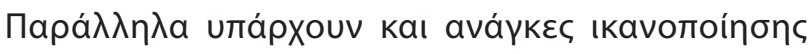

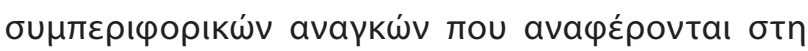

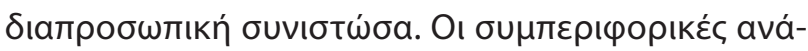

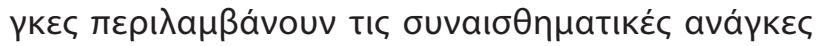

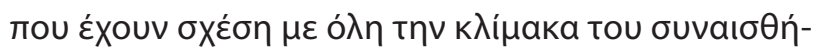

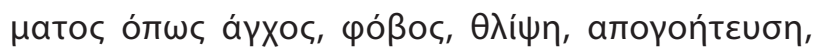

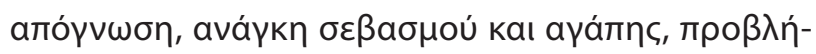

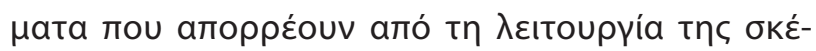

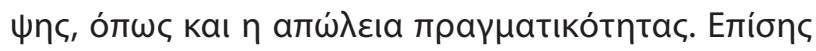

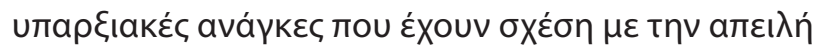

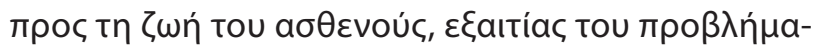

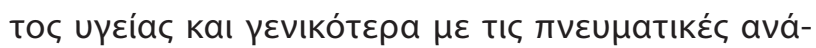

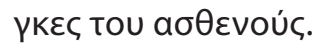

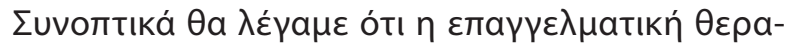

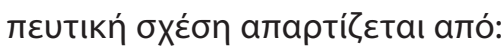

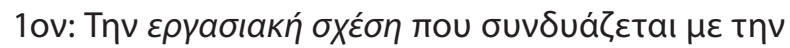

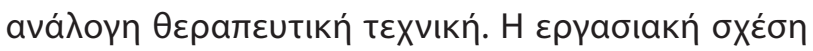

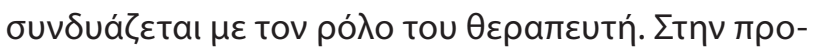

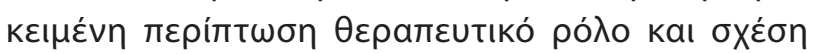

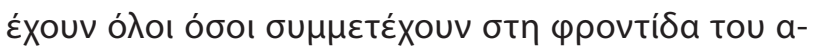

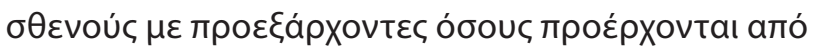

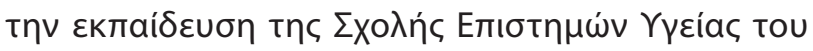

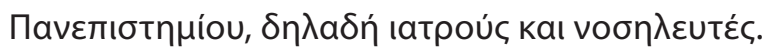

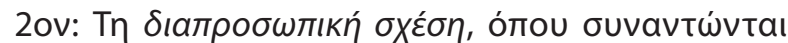

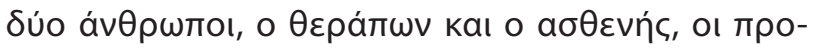

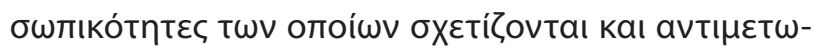

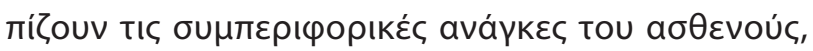

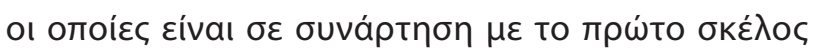

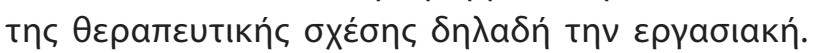

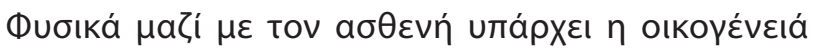

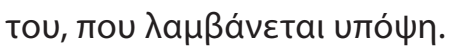




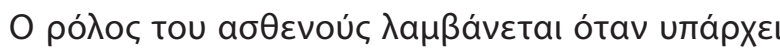

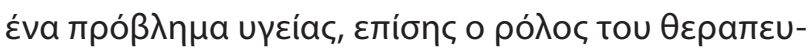

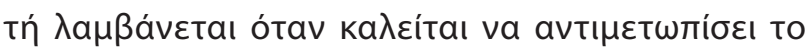

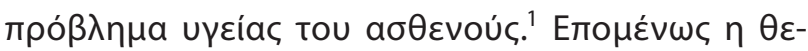

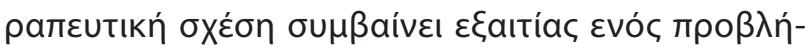

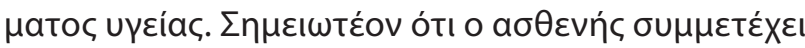

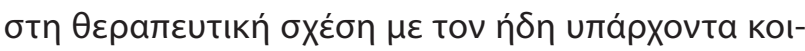

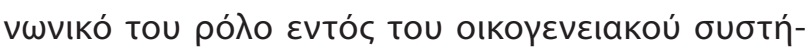
натос.

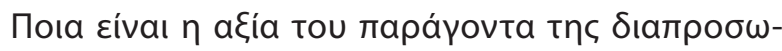

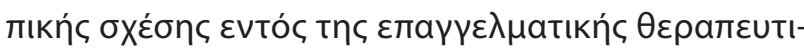

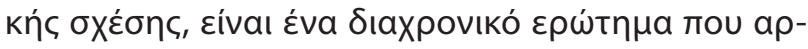

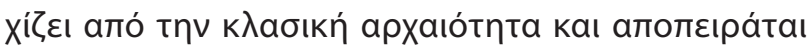

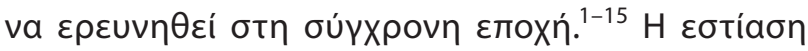

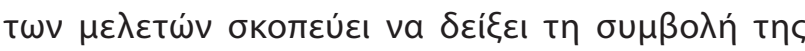

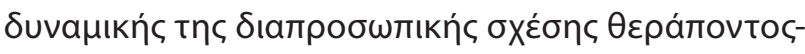

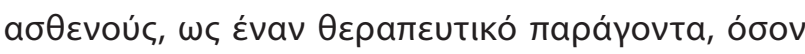

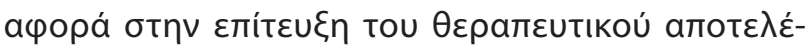
бнатос.

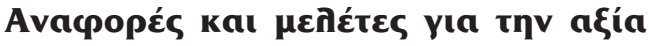

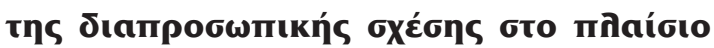

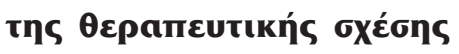

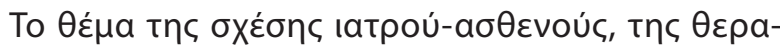

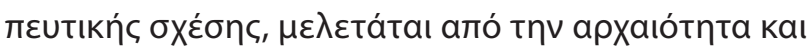

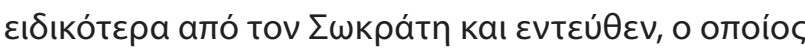

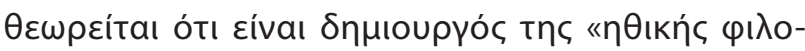

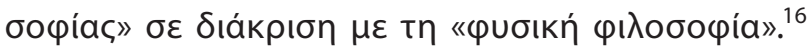

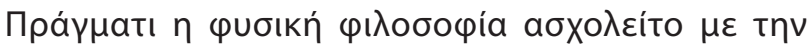

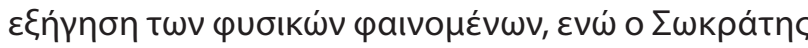

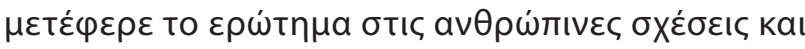

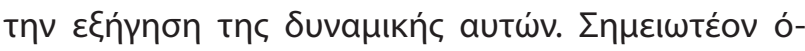

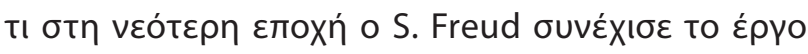

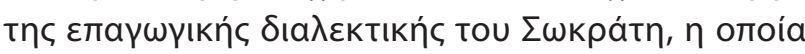

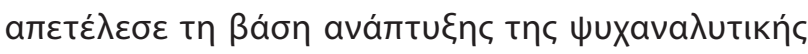

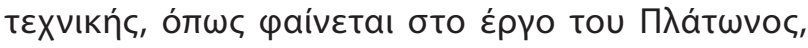

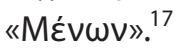

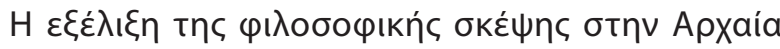

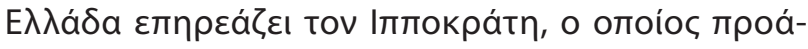

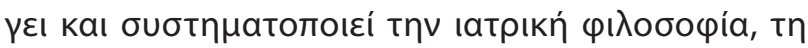

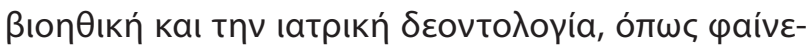

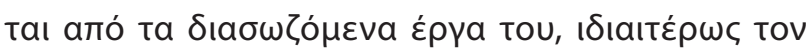

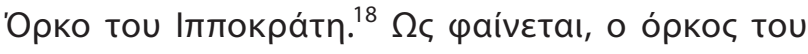

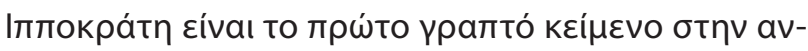

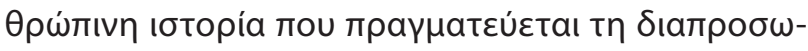

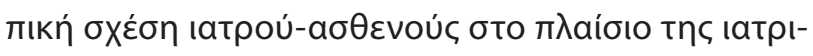
kńs áбknons.

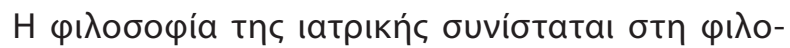

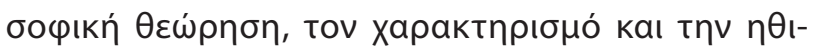

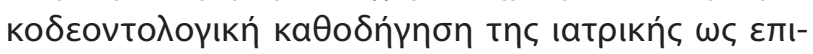

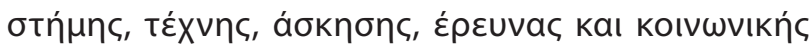

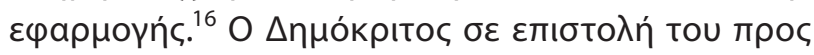

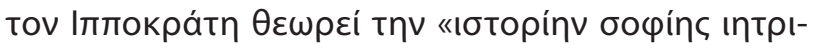

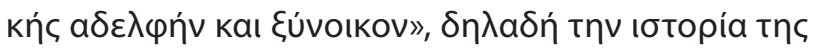

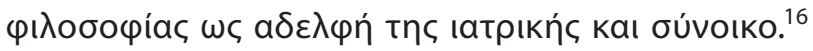

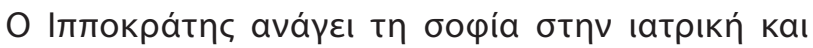

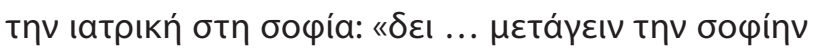

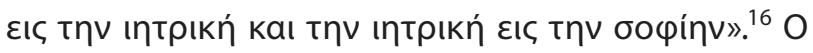

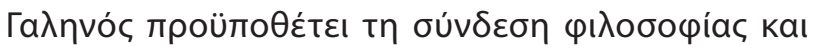

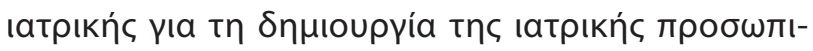

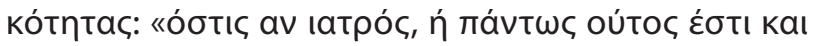

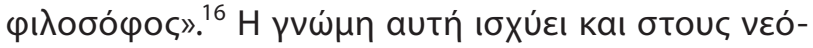

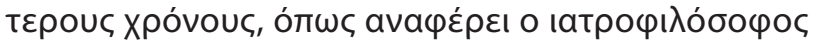

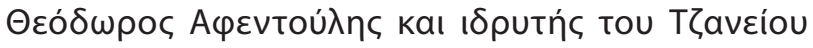

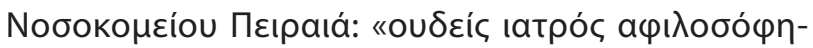
TO५»). ${ }^{16}$

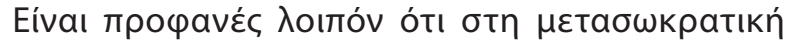

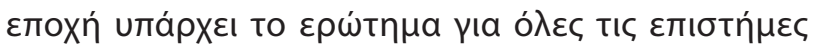

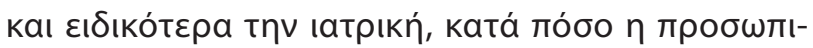

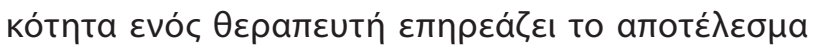

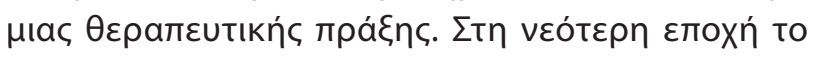

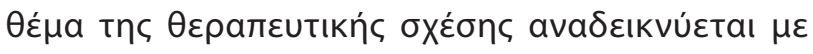

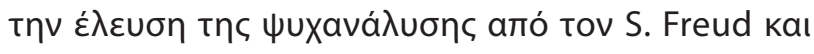

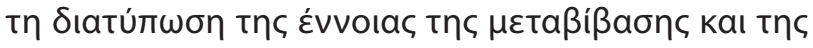

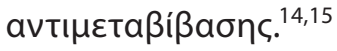

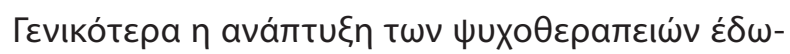

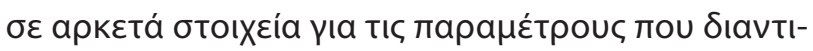

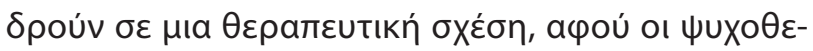

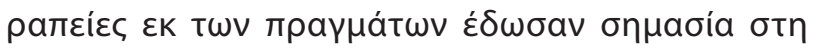

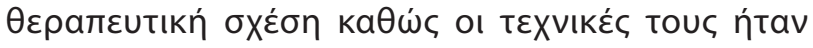

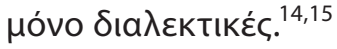

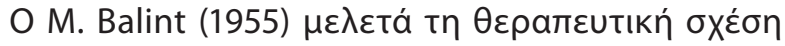

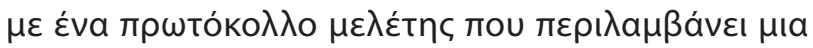

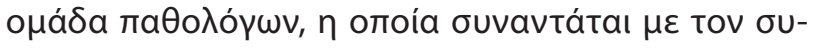

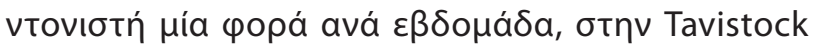

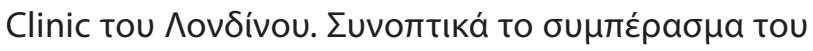

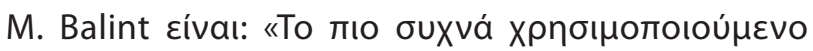

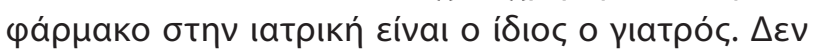

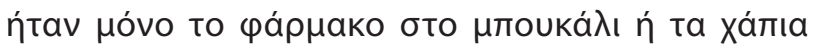

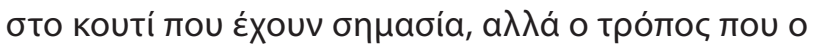

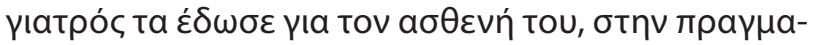




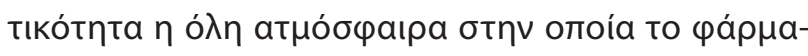

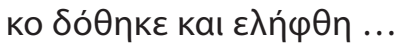

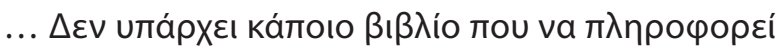

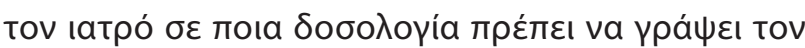

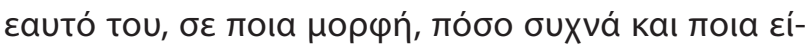
vaı $\eta$ Ө

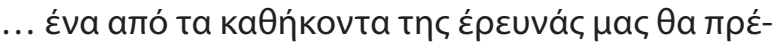

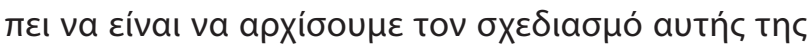

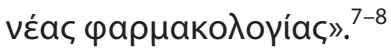

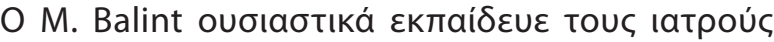

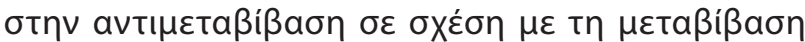

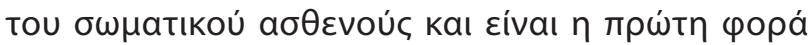

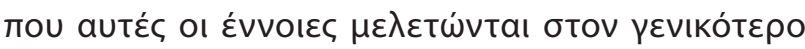

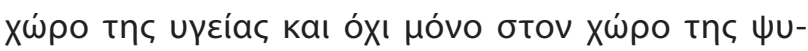

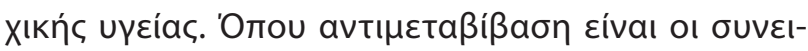

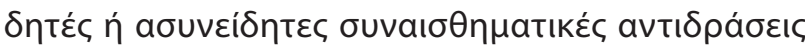

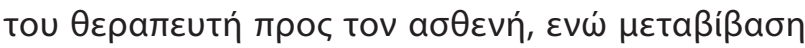

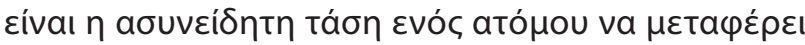

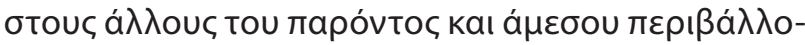

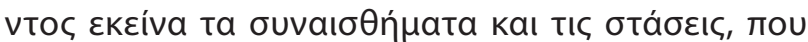

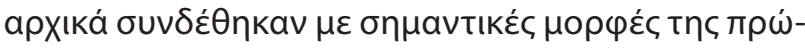

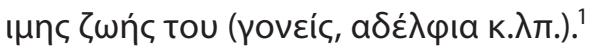

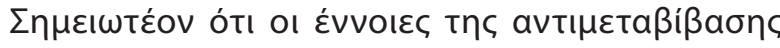

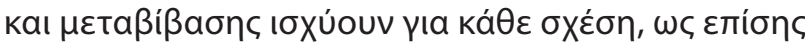

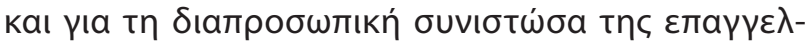

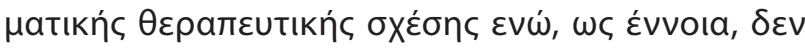
a

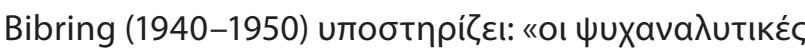

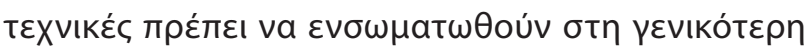

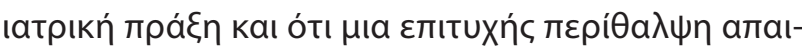

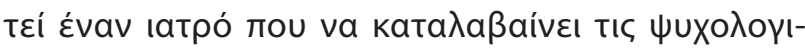

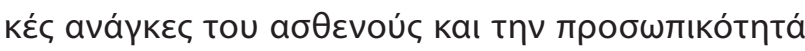
Tou». ${ }^{9}$

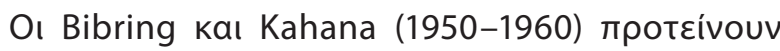

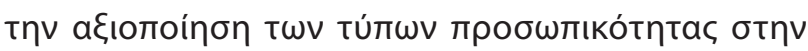

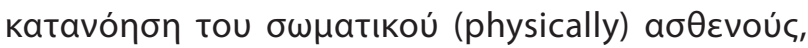

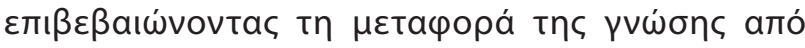

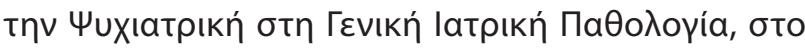

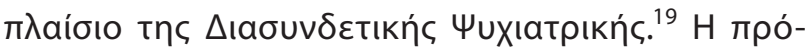

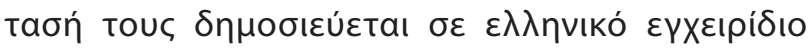

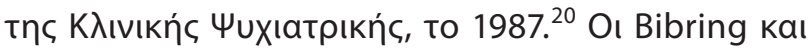

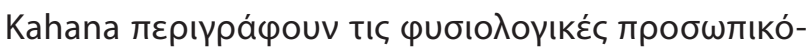

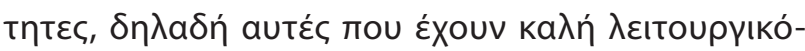

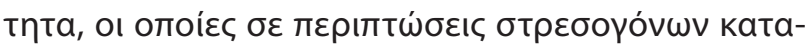

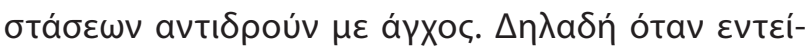

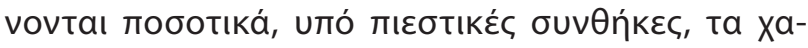

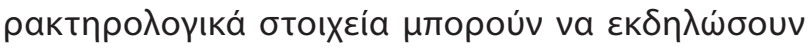

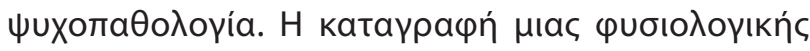

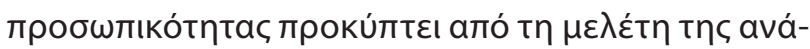

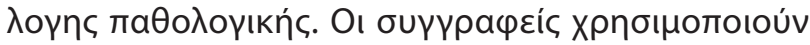

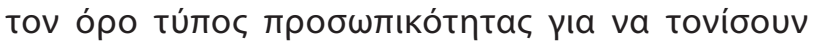

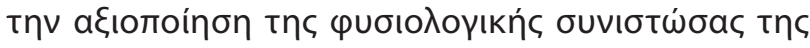

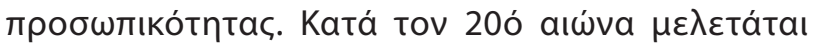

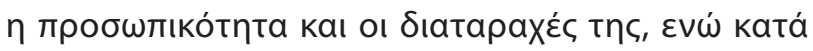

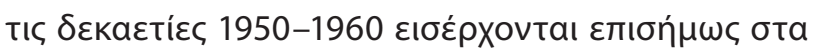

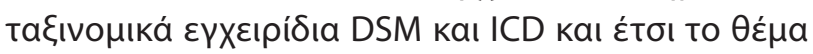

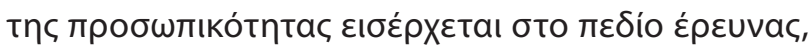

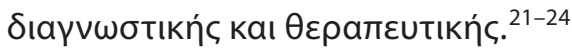

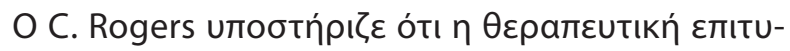

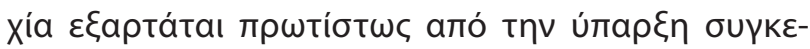

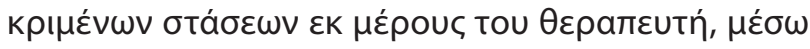

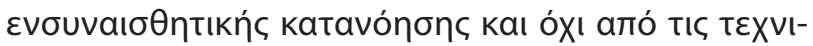

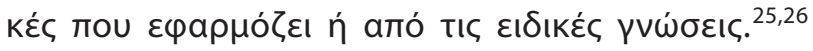

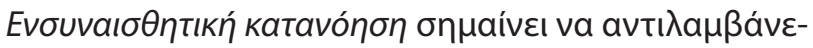

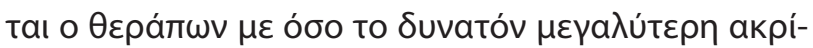

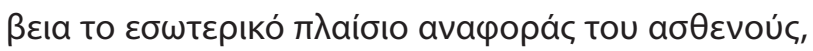

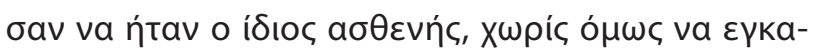

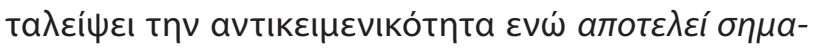

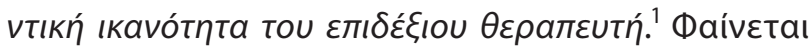

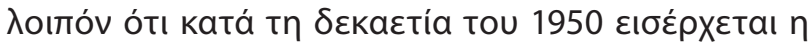

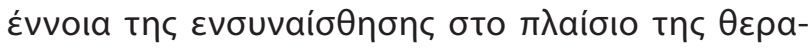

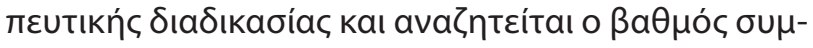

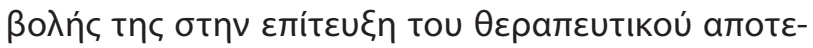
$\lambda \varepsilon \dot{\sigma}$ натос.

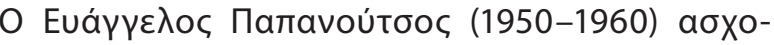

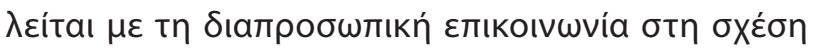

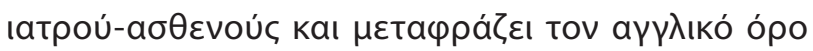

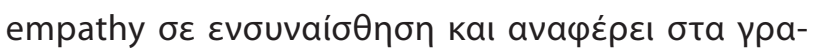

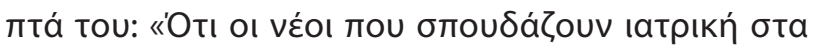

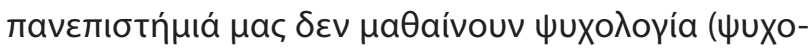

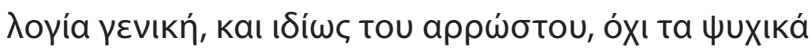

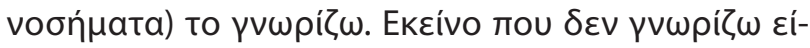

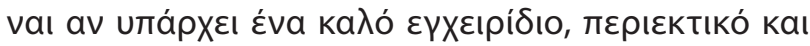

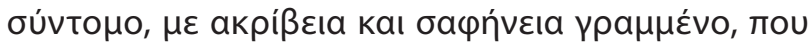

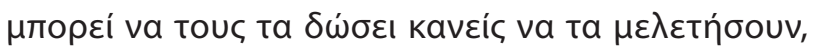

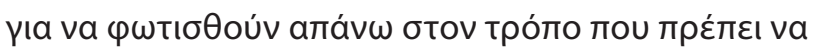

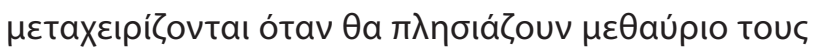

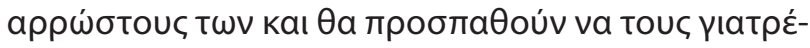
सOuv». ${ }^{27,28}$

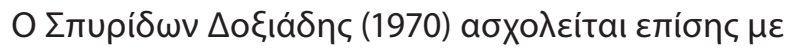

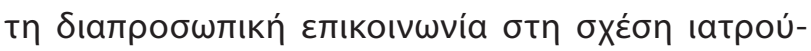

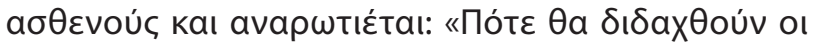




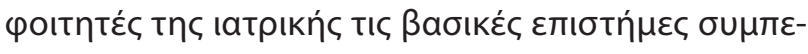

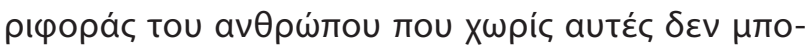

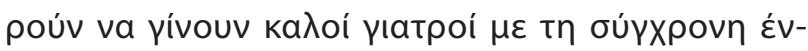

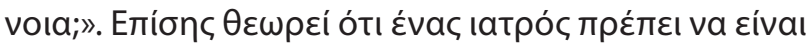

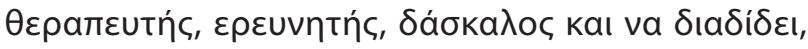

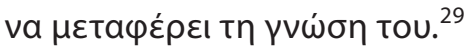

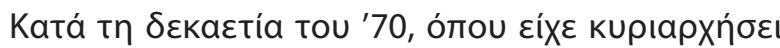

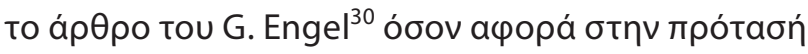

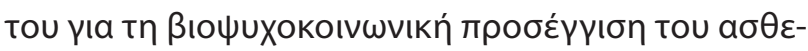

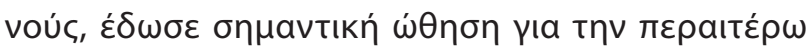

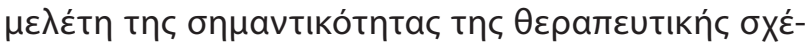

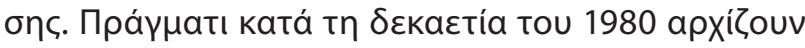

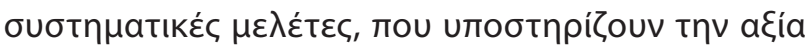

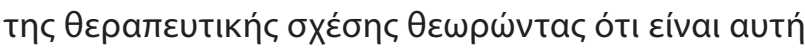

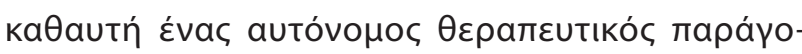

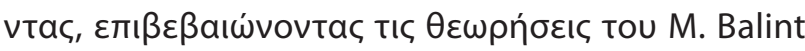
kal tou Rogers. ${ }^{30}$

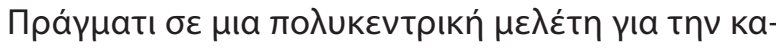
тá $\theta \lambda ı \psi \eta$ aró to National Institute of Mental Health Treatment of Depression Collaborative Research

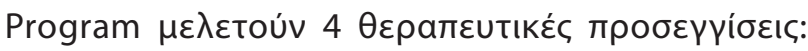

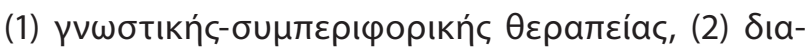

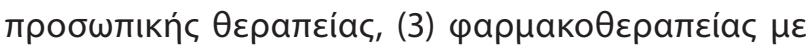

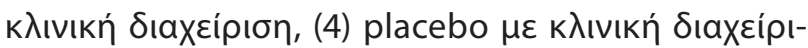

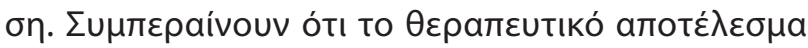

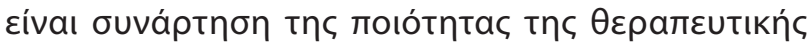

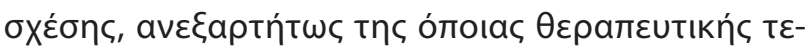
XVIKńc. ${ }^{31,32}$

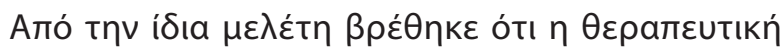

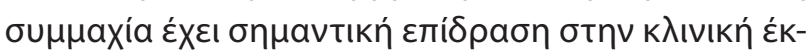

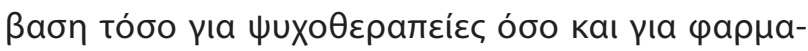

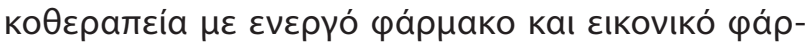

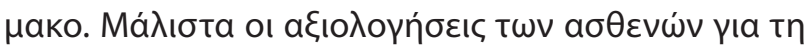

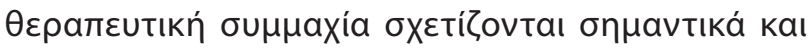

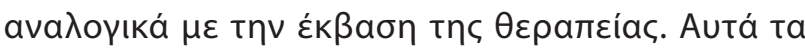

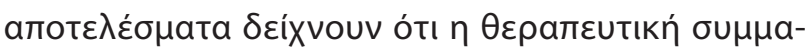

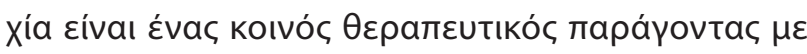

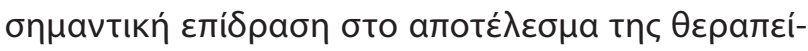

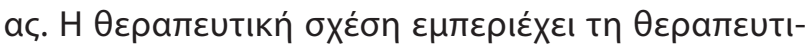

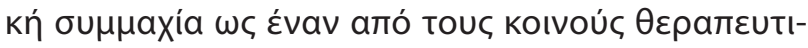

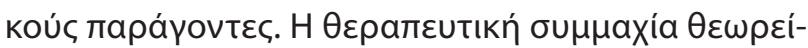

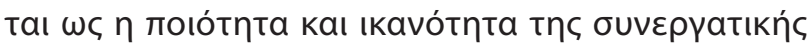

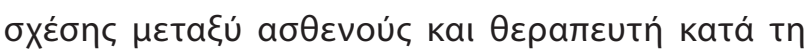

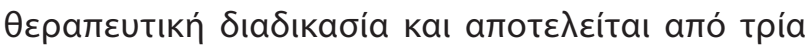

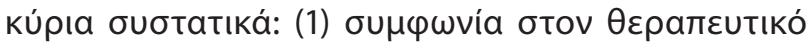

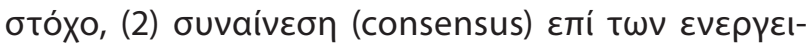

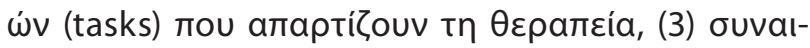

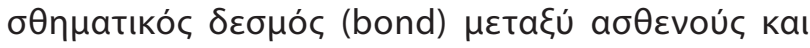

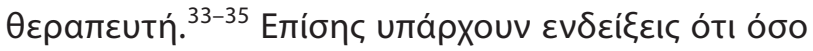

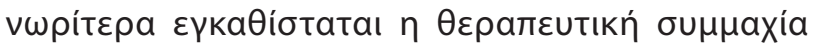

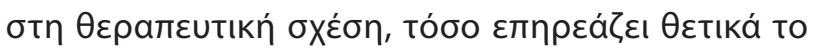
Ө

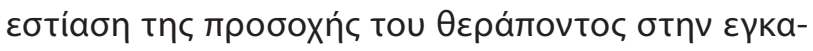

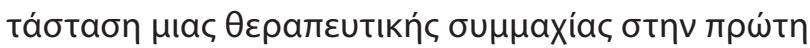

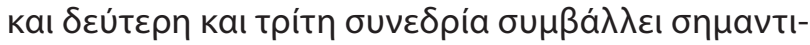

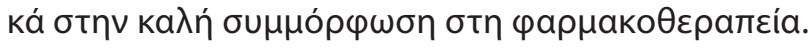

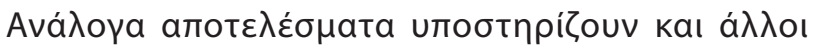

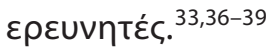

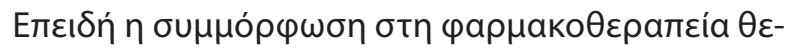

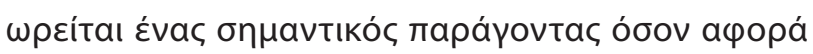

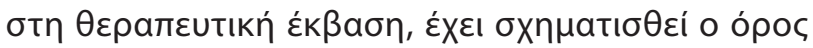

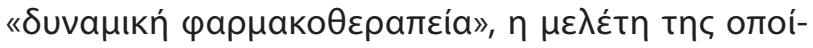

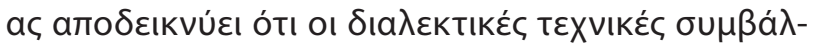

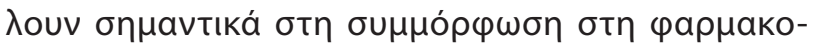

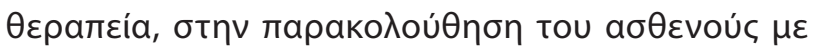

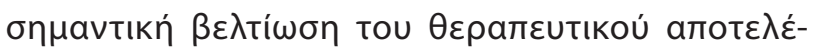

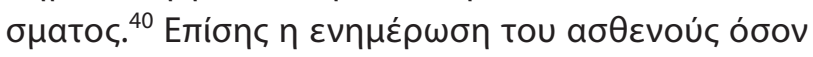

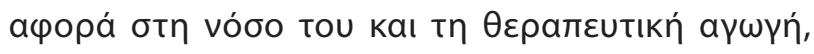

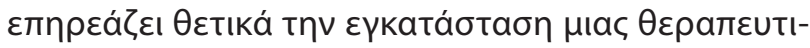

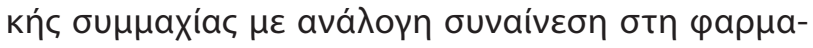

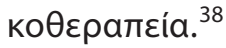

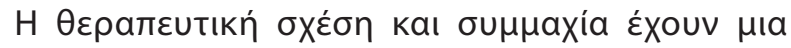

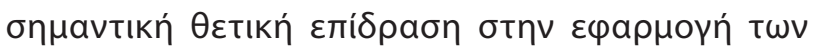

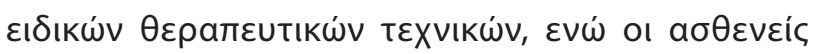

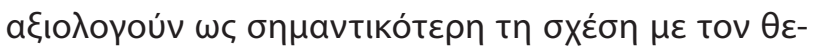

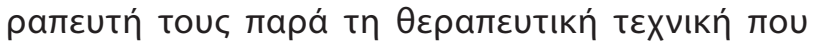

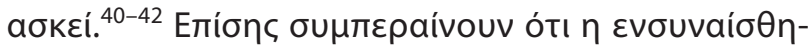
on (empathy), $\eta \mu \eta$ ктптікń $\theta \dot{\rho} \mu \eta \eta$ (non-possessive warmth), o $\theta \varepsilon \tau$ เाкóc $\sigma \varepsilon \beta a \sigma \mu o ́ c$ (positive regard) kaı $\eta$

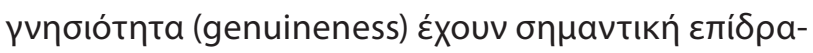

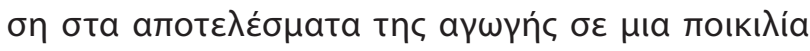

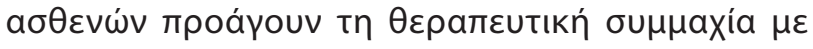

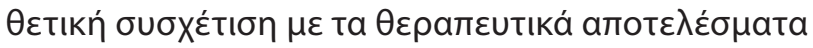

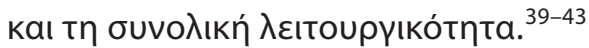

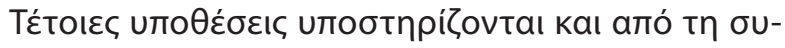

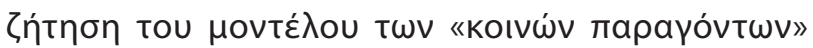

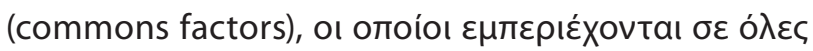

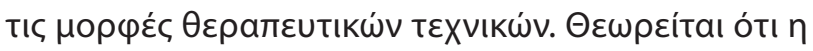

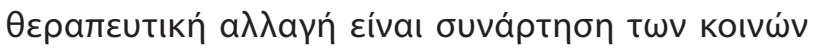

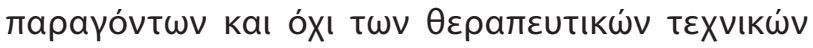




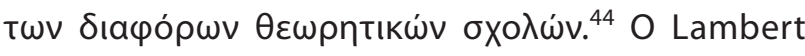

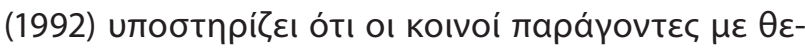

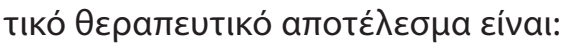

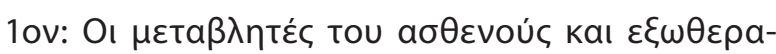

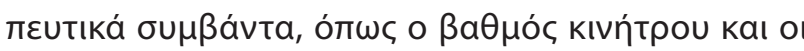

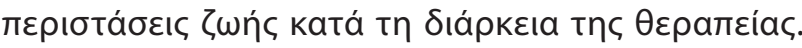

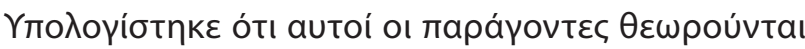

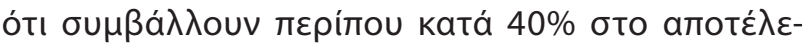

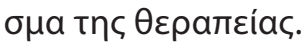

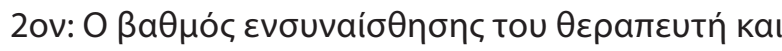

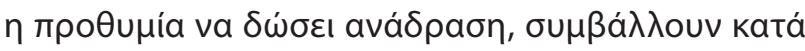

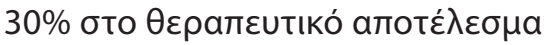

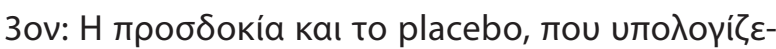

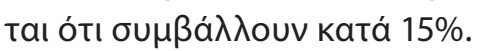

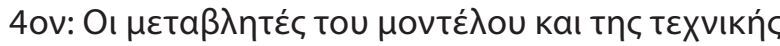

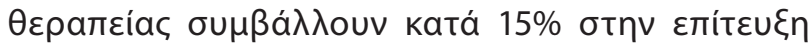

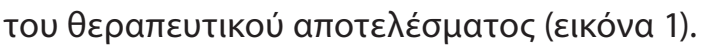

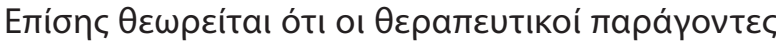

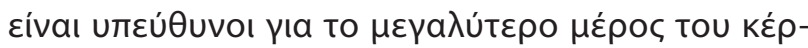

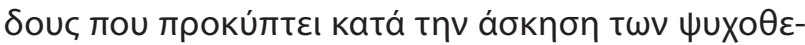

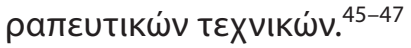

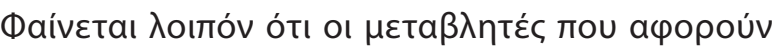

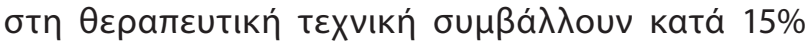

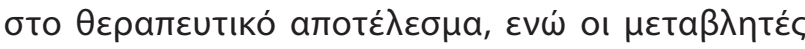

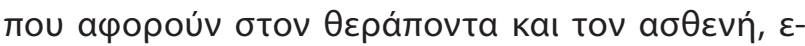

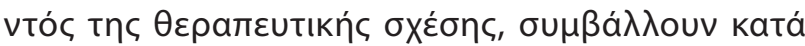
$85 \%$.

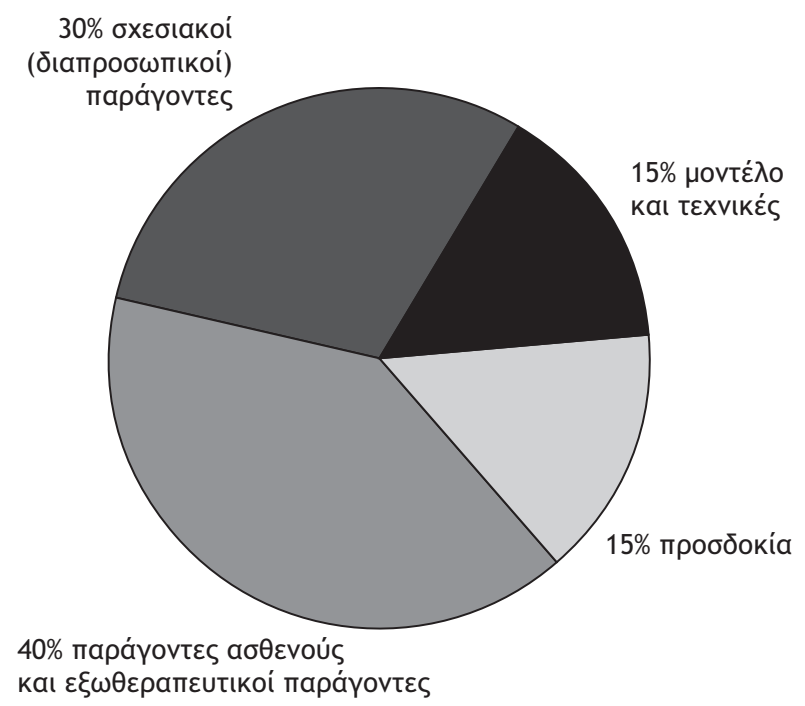

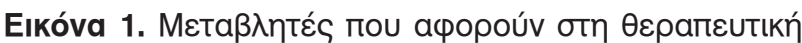

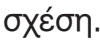

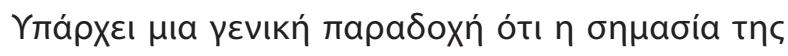

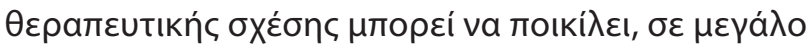

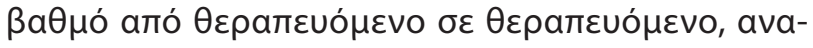

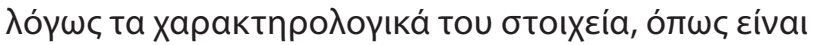

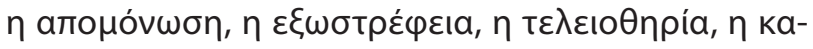

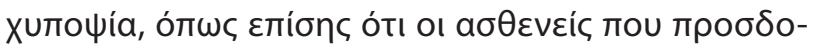

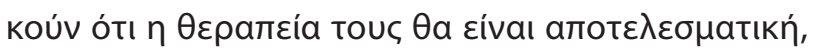

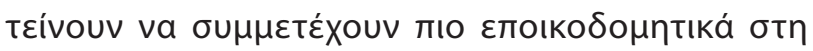

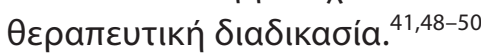

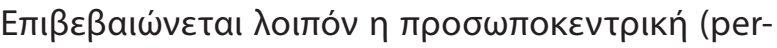

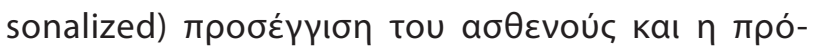

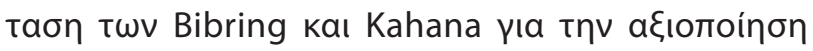

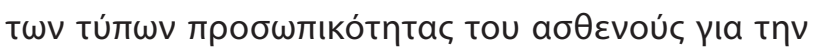

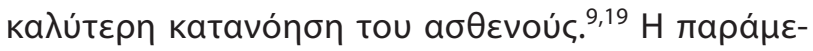

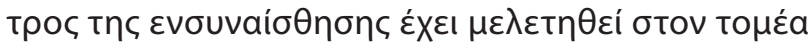

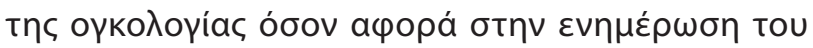

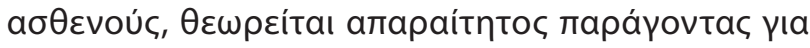

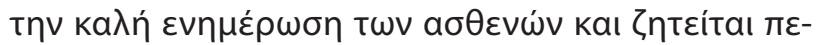

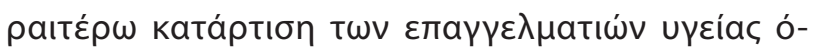

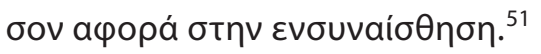

\section{Emínoyos}

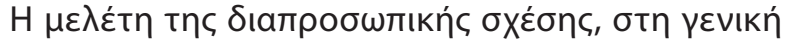

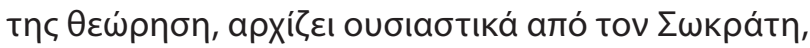

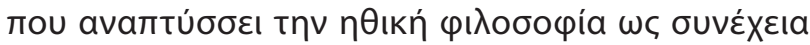

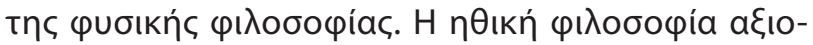

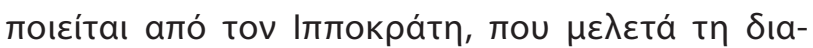

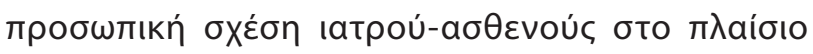

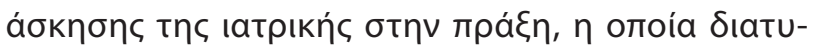

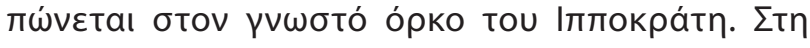

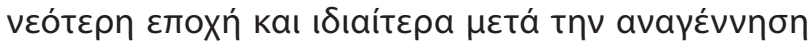

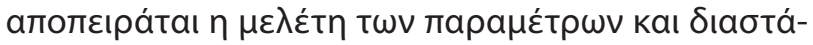

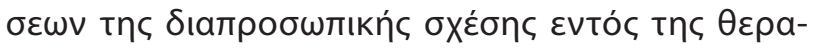

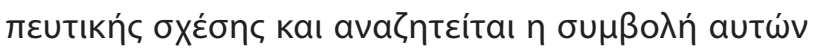

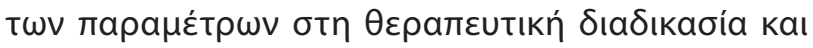

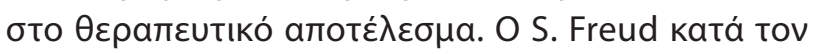

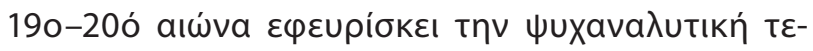

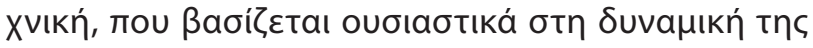

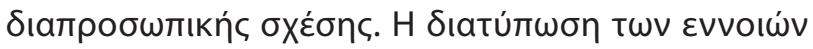

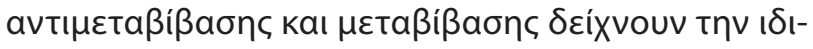

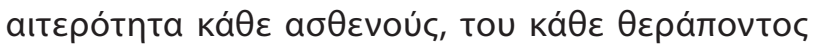

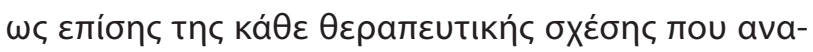

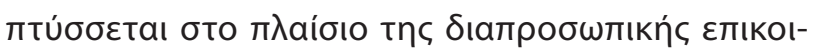

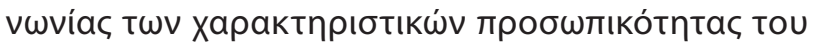

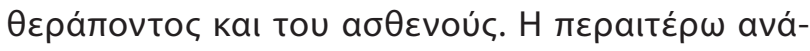

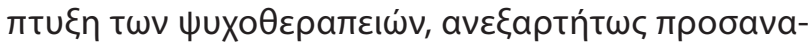




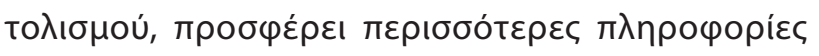

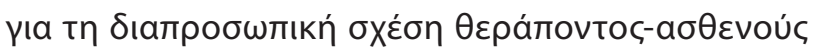

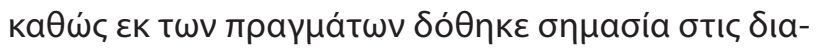

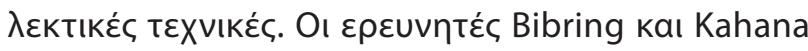

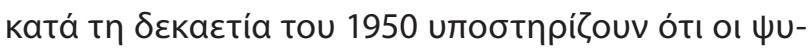

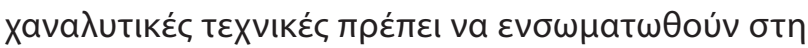

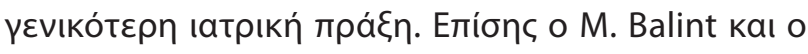

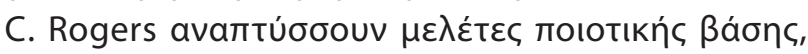

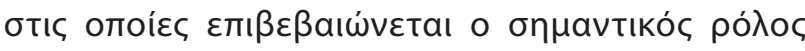

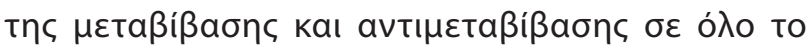

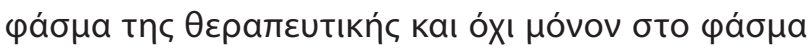

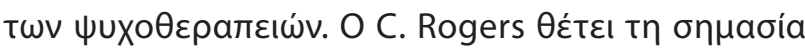

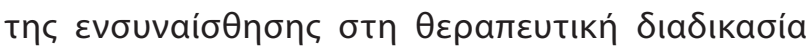

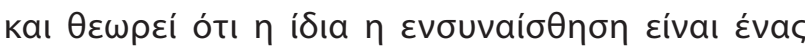

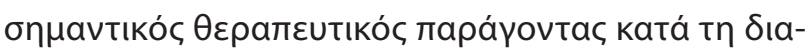

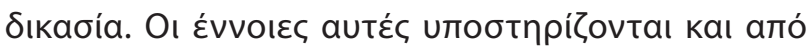

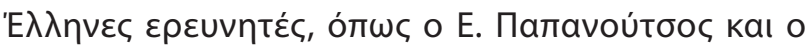

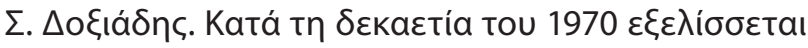

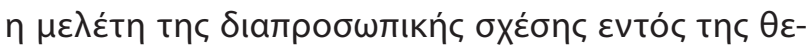

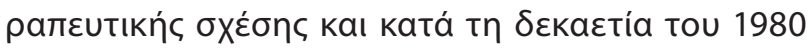

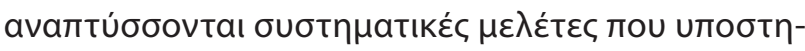

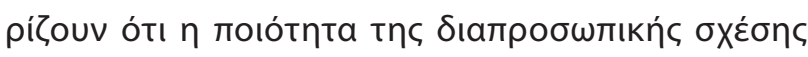

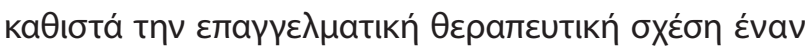

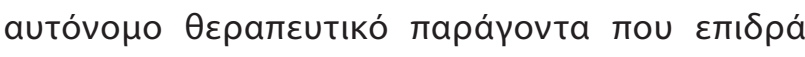

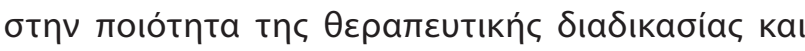

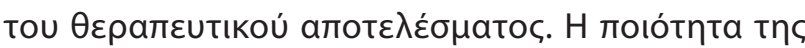

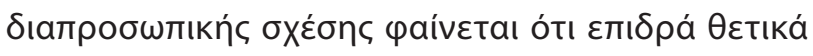

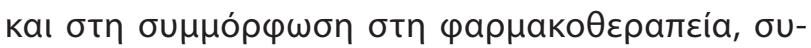

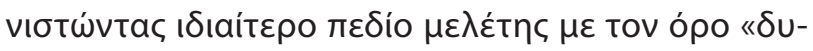

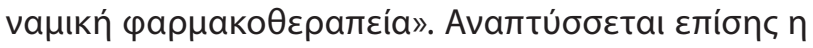

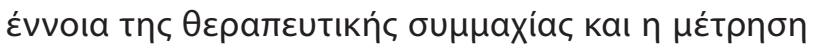

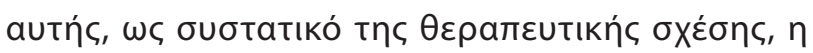

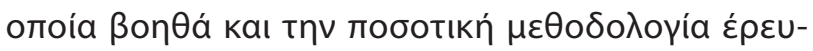

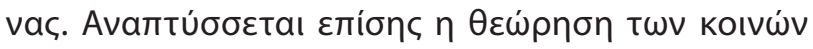

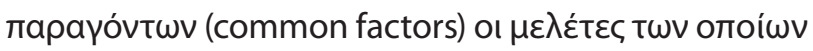

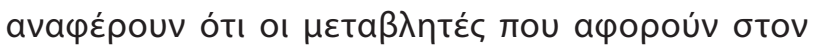

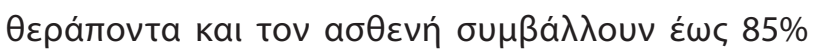

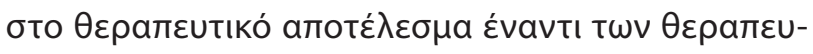

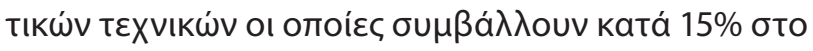

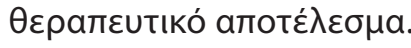

Oı $\mu \varepsilon \lambda \varepsilon \dot{t \varepsilon \varsigma, ~ \sigma u v \varepsilon x i ́ \zeta o v t a ı ~ k a ı ~ a v a \delta u ́ o u v ~ o ́ \lambda o ~ к a ı ~ \pi \varepsilon-~}$

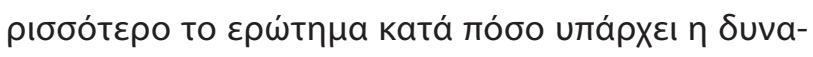

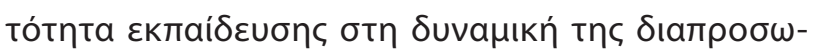

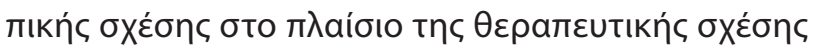

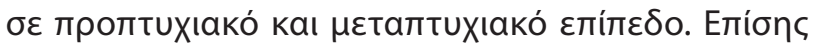

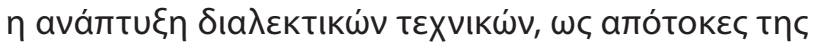

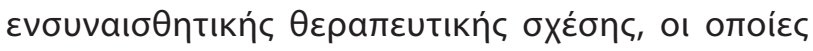

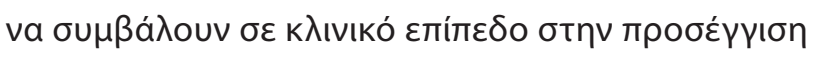

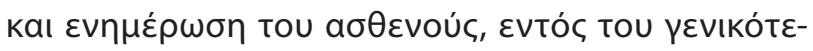

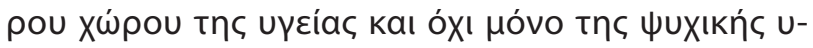
үé́ac.

\title{
The contribution of the relationship between therapist-patient and the context of the professional relationship
}

\author{
G. Kallergis \\ School of Health Sciences, Faculty of Nursing, National and Kapodistrian University of Athens, \\ Department of Psychiatry, "Agii Anargyroi Hospital", Athens, Greece
}

Psychiatriki 2019, 30:165-174

The therapeutic relationship is the common place of all medical specialties in therapeutic practice. It is a professional relationship and consists of two components: the work component and the interpersonal component. The focus of the studies aims to show the contribution of the dynamics of the therapist - patient interpersonal relationship as a therapeutic factor in achieving the therapeutic 
outcome. The issue of doctor-patient relationship has been studied since antiquity, in particular by Socrates and beyond. Hippocrates promotes and systematizes medical philosophy, bioethics and medical ethics, as seen in the well-known "Hippocratic Oath". In the new era, S. Freud continued the work of inductive dialectics of Socrates, while formulating the concept of transference and countertransference. The development of psychotherapies has provided enough evidence for the parameters that interact into a therapeutic relationship, as their techniques were merely dialectical. $M$. Balint supports the value of counter-transference and transference to the therapeutic relationship. G. Bibring \& R. Kahana suggest that psychoanalytic techniques and personality types contribute to the understanding of the physical patient. $C$. Rogers suggested that the attitudes and the empathic understanding of the therapist, not the techniques, contribute primarily to therapeutic success. G. Engel (1970) promotes the patient's biopsychosocial approach. Since 1980, systematic studies have begun to support the value of the therapeutic relationship, believing that it is itself an autonomous therapeutic factor, confirming the views of M. Balint and C. Rogers. They conclude that the therapeutic effect is a function of the quality of the therapeutic relationship, regardless of any therapeutic technique, and that the therapeutic alliance has a significant effect on the clinical outcome for psychotherapies as well as for pharmacotherapy. Empathy, non-possessive warmth, positive respect and authenticity have a significant effect on the treatment results. The common factor model supports the dynamics of the interpersonal relationship contributing $85 \%$ to the therapeutic effect whereas the therapeutic techniques contribute $15 \%$. It therefore seems that the dynamics of the interpersonal relationship, the therapist-patient, is an important therapeutic factor. Studies continue and more questions arise as to whether education is available, the dynamics of interpersonal relationships in the context of therapeutic relationships at undergraduate and postgraduate levels. Also, the development of dialectical techniques, as a response to the empathic therapeutic relationship, which contributes at the clinical level to the patient's approach and information within the general health area and not only to mental health.

Key words: Interpersonal relationship, therapeutic relationship, therapeutic factor, empathy, countertransference.

\section{BıBAtoypacpía}

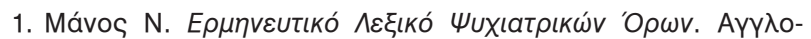

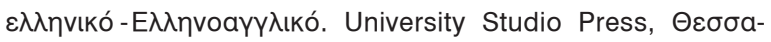

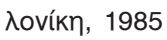

2. Greenson RR. The working alliance and the transference neurosis. In: Explorations in Psychoanalysis. International Universities Press. New York, 1978:119-224

3. Greenson RR. The Technique and Practice of Psychoanalysis. International Universities Press, New York, 1967

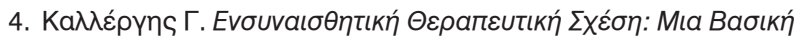

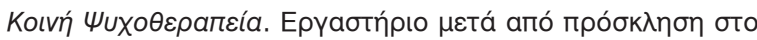

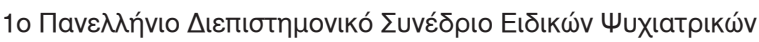

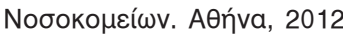

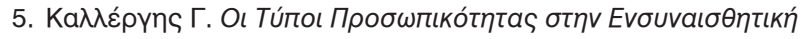

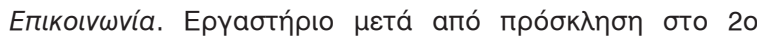

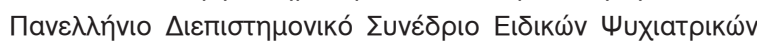

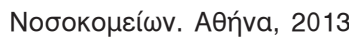

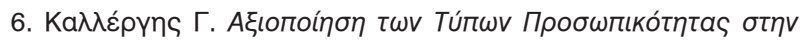

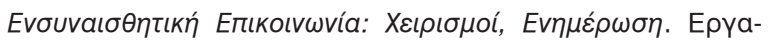

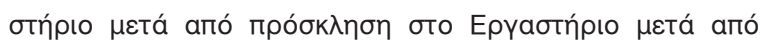

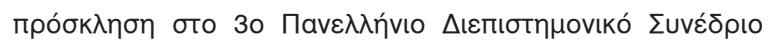

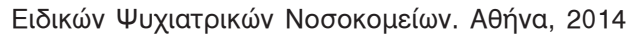

7. Balint M. The Doctor, His patient and the IIIness. 2nd Ed. Churchill, Livingstone, 1990

8. Balint M. Problems of Human Pleasure and Behaviors. Maresfield Library, London, 1957

9. Bibring GL. The teaching of Dynamic Psychiatry. International Universities Press, USA, 1968

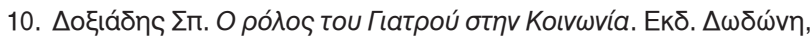
AӨńva, 1990

11. Rogers C. On Becoming a Person: A Therapist's View of Psychotherapy. Houghton Mifelin Company, Boston, 1961

12. Rogers C. Client-Centered Therapy: Its Current Practice, Implications and Theory. Constable, London, 1951

13. Rogers C. A Theory of Therapy, Personality and Interpersonal Relationships as Developed in the Client-centered Framework. McGraw Hill. New York, 1959 
14. Freud S. The dynamics of transference. In: J. Starchey (ed) The standard edition of the complete psychological works of Sigmund Freud. Hogarth Press, London, 1912/1958

15. Freud S. On the beginning of treatment: Further recommendations of the technique of psychoanalysis. In: J. Starchey (ed) The standard edition of the complete psychological works of Sigmund Freud. Hogarth Press, London, 1912/1958

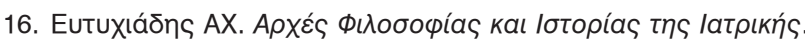

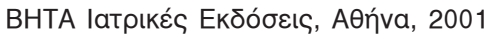

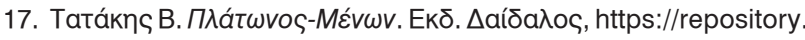
edulll.gr/edulli/retrieve/7024/1829_menon.pdf

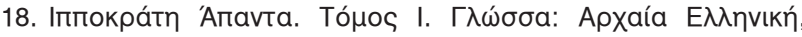
Епцел入.: D. Carolus Gottlom Kühn, Lipsiae, 1825

19. Bibring GL, Kahana RJ. Lectures in medical Psychology: An Introduction to the Care of Patients. Arch Gen Psychiatry 1969, 21:638-639, doi:10.1001/archpsyc.1969.01740230126027

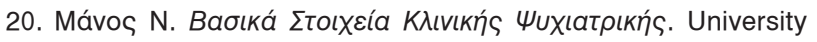

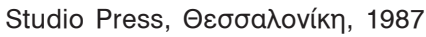

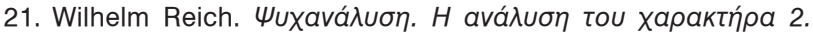

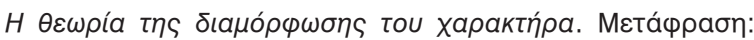

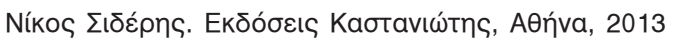

22. Schneider K. Psychopath ische Persönlichkeiten. In: Klinische Psychopathologie. 11. Unveränderte Auflage. Stuttgart, Germany: Thieme Verlag, 1976:17-39

23. Sıүvwotıká Koıtńpıa anó DSM-5. Desk Reference To The

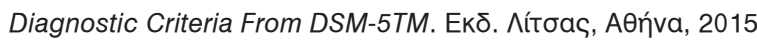

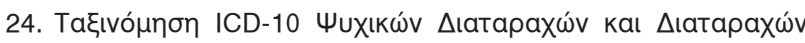

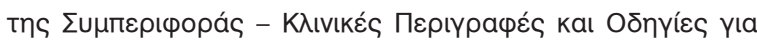

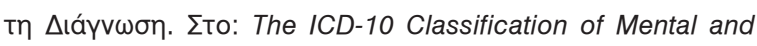

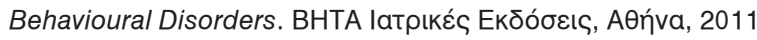

25. Rogers CR. Empathic: An unappreciated way of being. Counseling Psychologist 1975, 5:2-11, doi: 10.1177/00110000 7500500202

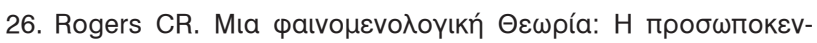

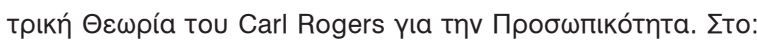

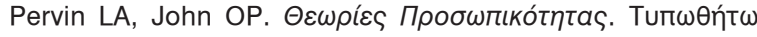
- Гı́́pүos $\Delta$ apঠavós, AӨńva, 2001

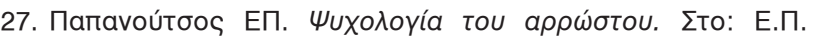

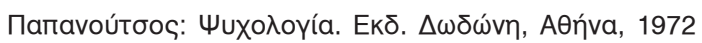

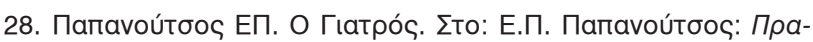

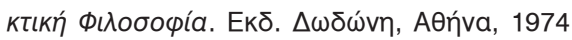

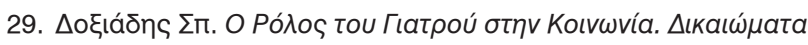

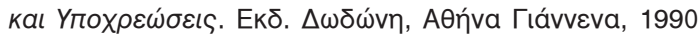

30. Engel GL. The need for a new medical model: a challenge for biomedicine. Science 1977, 196:129-136, PMID: 847460

31. Elkin I, Pilkonis PA, Docherty JP, Sotsky SM. Conceptual and methodological issues in comparative studies of psychotherapy and pharmacotherapy active ingredients and mechanisms of change. Am J Psychiatry 1988, 145:909-917, doi:10.1176/ajp.145.8.909

32. Krupnick JL, Sotsky SM, Simmens S, Moyer J, Elkin I, Watkins $\mathrm{J}$ et al. The role of the therapeutic alliance in psychotherapy and pharmacotherapy outcome: findings in the National Institute of Mental Health Treatment of Depression
Collaborative Research Program. J Consult Clin Psychol 1996, 64:532-539, PMID: 8698947

33. Howard K, Rickels K, Mock JE, Lipman RS, Covi L, Baumm NC. Therapeutic style and attrition rate from psychiatric drug treatment. J Nerv Ment Dis 1970, 150:102-110, doi: 10.1097/ 00005053-197002000-00002

34. Wright JH, Davis D. The therapeutic relationship in cognitive-behavioral therapy: Patient perceptions and therapist responses. Cognit Behav Pract 1994, 1:25-45, doi: 10.1016/ S1077-7229 (05)80085-9

35. Bordin E. Theory and research on the therapeutic working alliance: New directions. In: Horvath A, Greenberg L (eds) The working alliance: Theory, research and practice. Wiley, New York, 1994

36. Docherty JP, Fiester SJ. The therapeutic alliance and compliance with psychopharmacology. In: American Psychiatric Association (ed) Psychiatry update 1985, 4:607-632

37. Elkin I, Pilkonis PA, Docherty JP, Sotsky SM. Conceptual and methodological issues in comparative studies of psychotherapy and pharmacotherapy: active ingredients and mechanisms of change. Am J Psychiatry 1988, 145:909-917, doi: 10.1176/ajp.145.8.909

38. Gutheil TG. The psychology of psychopharmacology. Bull Menninger Clin 1982, 46:321-330, PMID: 7139146

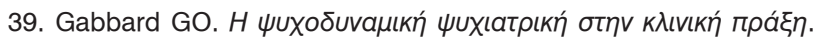

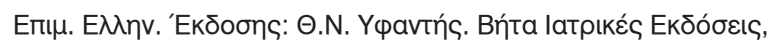
AӨńva, 2006

40. Keijsers GP, Schaap CP, Hoogduin CA. The impact of interpersonal patient and therapist behavior on outcome in cognitive-behavior therapy. A review of empirical studies. Behav Modif 24:264-297, doi: 10.1177/0145445500242006

41. Bohart AC, Elliott R, Greenberg LS, Watson JC. Empathy. In: Norcross JC (ed) Psychotherapy relationships that work: Therapist contributions and responsiveness to patients. Oxford University Press, New York, 2002

42. Miller A. Systems theory applied to the work of an educational psychologist. Assoc Educat Psycholog J 1980, 5:11-16

43. Castonguay LG1, Goldfried MR, Wiser S, Raue PJ, Hayes AM. Predicting the effect of cognitive therapy for depression: a study of unique and common factors. J Consult Clin Psychol 1996, 64:497-504, PMID: 8698942

44. Lambert MJ. Psychotherapy outcome research: implications for integrative and eclectic therapists. In: Norcross JC, Goldfried MR (eds) Handbook of psychotherapy integration. 1st ed. Basic Books, New York, 1992

45. Hubble MA, Duncan BL, Miller SD. The Heart and Soul of Change: What Works in Therapy. American Psychological Association. Washington DC, 1999

46. Asay TR, Lambert MJ. The empirical case of the common factors in psychotherapy: quantitative findings. In: M.A. Hubble, B.L. Duncan, S.D. Miller (eds) The heart and soul of change: what works in therapy. American Psychological Association. Washington DC, 1999, doi: 10.1037/11132-001 
47. Lambert MJ. The Handbook of Psychology Integration. Basic Books, New York, 1992

48. Beutler LE. The empirically supported treatments movement: A scientist-practitioner's response. Clin Psychol Sci Pract 2006, 3:225-229, doi: 10.1093/clipsy.bph076

49. Norcross JC, Lambert ML. Psychotherapy Relationships That Work II. Psychotherapy 2011, 48:4-8, doi: 10.1037/a0022 180

50. Blatt SJ, Quinlan DM, Zuroff DC, Pilkonis PA. Interpersonal factors in brief treatment of depression: further analyses of the National Institute of Mental Health Treatment of Depression Collaborative Research Program. J Consult Clin Psychol 1996 64:162-171, doi: 10.1037/0022-006X.64.1.162
51. Baile WF, Buckman R, Lenzi R, Glober G, Beale EA, Kudelka AP. SPIKES- A six step protocol for delivering bad news: Applications to the patient with cancer. The Oncologist 2000, 5:302-311, PMID: 10964998

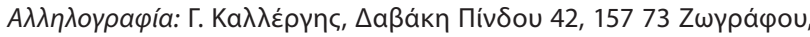

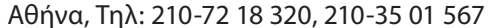

e-mail: gkallerg@nurs.uoa.gr 


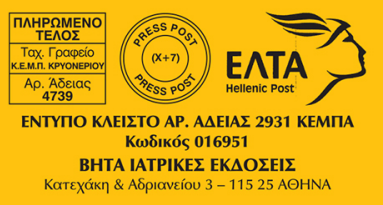

Періобіко́ "Чихіатріки́"

Alovuó́ou Alyivítou 17, 3os ópoøos, 11528 A日ńva

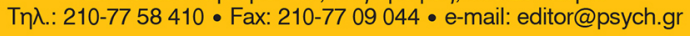

The Journal "Psychiatriki"

17, Dionisiou Eginitou str., 3rd floor, 11528 Athens, Greece

Tel.: (+30) 210-77 58410 • Fax: (+30) 210-210-77 09044 • e-mail: editor@psych.gr

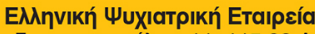

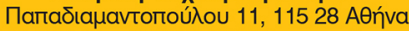

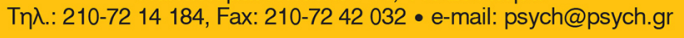

Hellenic Psychiatric Association

11, Papadiamantopoulou str., 11528 Athens, Greece

Tel.: (+30) 210-72 14 184, Fax: (+30) 210-72 42032 • e-mail: psych@psych.gr 